

\section{Propositions}

1. Operating an activated carbon filter for maximum bioregeneration counteracts micropollutants adsorption.

(this thesis)

2. Quantifying micropollutants degradation in activated carbon filters is key to understanding the potential and limitations of bioregeneration. (this thesis)

3. Evaluating scientific performance based on number of publications discourages breakthroughs in science.

4. Technological developments can only to a limited extent expand the carrying capacity of the environment.

5. The environmental damage for the production of goods should be incorporated in the price consumers pay for their products.

6. Social distancing reduces one's resilience.

7. Success is more determined by chance than by merit.

Propositions belonging to the thesis, entitled

Micropollutants removal with activated carbon: adsorption and regeneration by biodegradation

Laura Magalhães Piai, 5 February 2021 



\section{Micropollutants removal with activated carbon: adsorption and regeneration by biodegradation}

\section{Laura Magalhães Piai}




\section{Thesis committee}

\section{Promotor}

Prof. Dr A. van der Wal

Special Professor, Environmental Technology

Wageningen University \& Research

\section{Co-promotor}

Dr A.A.M. Langenhoff

Associate Professor, Environmental Technology

Wageningen University \& Research

\section{Other members}

Prof. Dr D.Z. Machado de Sousa, Wageningen University \& Research

Prof. Dr B. Barbeau, Polytechnique Montréal, Canada

Prof. Dr G.J.W. Euverink, University of Groningen

Prof. Dr J.P. van der Hoek, Delft University of Technology

This research was conducted under the auspices of the Graduate School for SocioEconomic and Natural Sciences of the Environment (SENSE) 


\title{
Micropollutants removal with activated carbon: adsorption and regeneration by biodegradation
}

\section{Laura Magalhães Piai}

\author{
Thesis \\ submitted in fulfilment of the requirements for the degree of doctor \\ at Wageningen University \\ by the authority of the Rector Magnificus, \\ Prof. Dr A.P.J. Mol, \\ in the presence of the \\ Thesis Committee appointed by the Academic Board \\ to be defended in public \\ on Friday 5 February 2021 \\ at 1:30 p.m. in the Aula.
}


Laura Magalhães Piai

Micropollutants removal with activated carbon: adsorption and regeneration by biodegradation, 208 pages

PhD thesis, Wageningen University, the Netherlands (2021)

With references, with summary in English and Portuguese

ISBN: 978-94-6395-636-9

DOI: $10.18174 / 536256$ 
To my family 



\section{Table of contents}

$\begin{array}{lll}\text { Chapter } 1 \text { General introduction } & 9\end{array}$

Chapter 2 Diffusion of hydrophilic organic micropollutants in

granular activated carbon with different pore sizes

Chapter 3 Biodegradation and adsorption of micropollutants by

biological activated carbon from a drinking water

production plant

Chapter 4 Melamine degradation to bioregenerate granular activated carbon

Chapter 5 Biodegradation of melamine in activated carbon filters resulting in bioregeneration

Chapter 6 General discussion

References

Summary I Resumo

Acknowledgements

About the author

Publications 


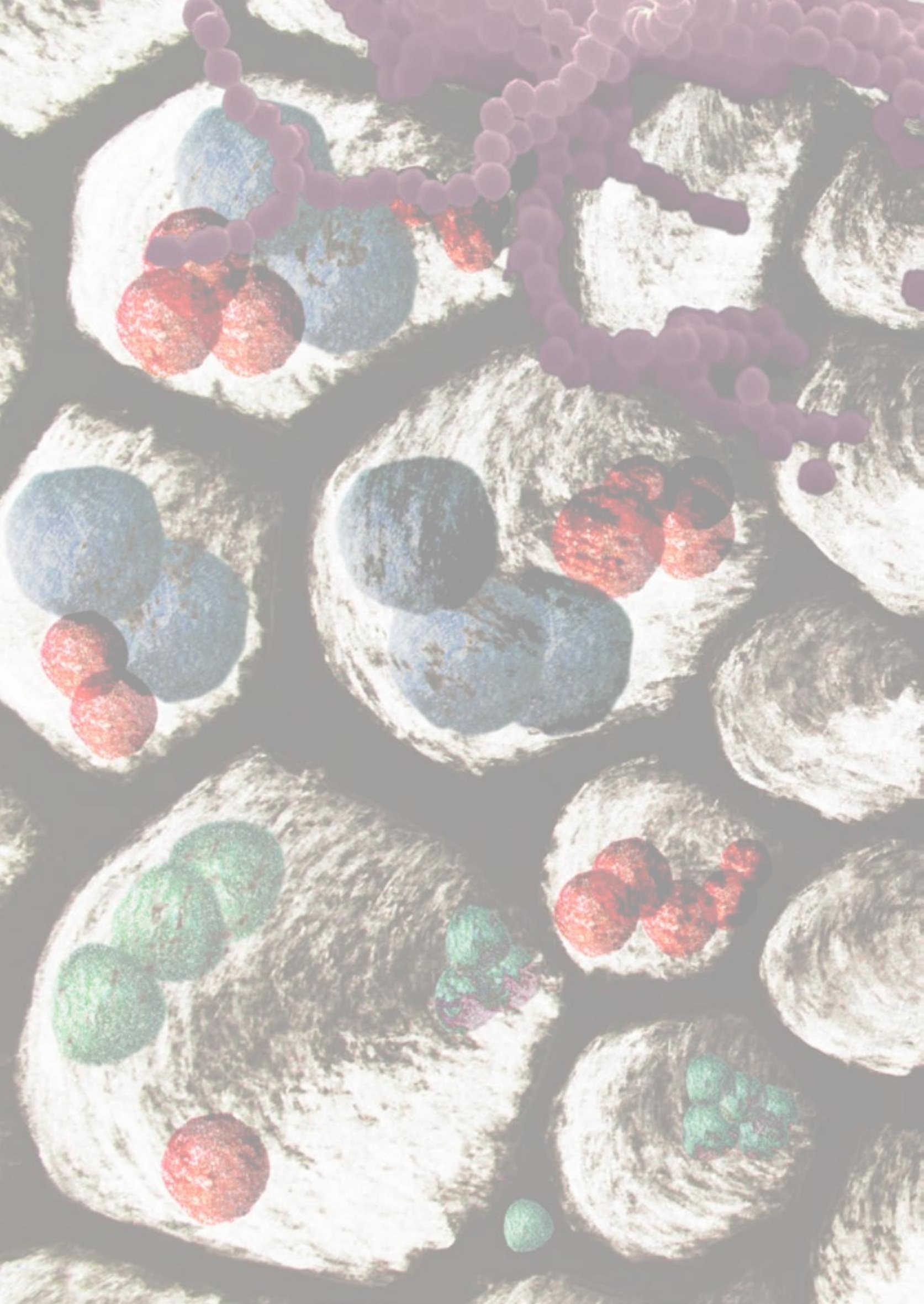




\subsection{General background}

Earth's human population is growing rapidly, increasing demand on clean water, whilst increasing pollution of water resources. Pollution of water resources further aggravates water stress. An emerging class of water pollutants has been identified in the last decades, originating from use of organic chemicals and their release to environmental compartments. One of the key differences between this emerging class of pollutants and conventional pollutants (e.g. nitrate, phosphorus and organic matter) is the large variety of chemical structures of the different contaminants and their occurrence at trace (ng/L to $\mu \mathrm{g} / \mathrm{L}$ ) concentrations. The latter is the reason why these emerging pollutants are also referred to as organic micropollutants. For conciseness, only the term micropollutants is used in this thesis. Despite their occurrence at trace concentrations, micropollutants contribute significantly to the total pollution load on the aquatic environment (Martínez-Alcalá et al., 2018; Wohler et al., 2020) and can be harmful to aquatic organisms (Santos et al., 2010).

\subsection{Organic micropollutants in the environment}

Throughout the world, an increasing number of studies show the presence of a large variety of micropollutants in surface water (Ruff et al., 2015; Schulze et al., 2019; Starling et al., 2019; Tröger et al., 2020; Xu et al., 2019), groundwater (Bexfield et al., 2019; Stuart et al., 2012) and to a lesser extent also in drinking water (Baken et al., 2018; Trenholm et al., 2006; Tröger et al., 2018). In the river Rhine, an important drinking water source in central Europe, almost 200 micropollutants have been detected in 2011 (Ruff et al., 2015). In the Netherlands, over 700 micropollutants were detected in surface and groundwater, drinking water and effluent from domestic and industrial wastewater treatment plants (WWTPs) (Sjerps et al., 2016). Also in other parts of the globe, studies highlight the widespread contamination of water resources with micropollutants (Chaves et al., 2020; Dalahmeh et al., 2020; Luo et al., 2014; Starling et al., 2019).

Micropollutants reach the environment via a variety of diffuse and point sources (Figure 1.1). Diffuse sources include agricultural and urban water runoff and sewer overflow (Christoffels et al., 2016; Launay et al., 2016; Seitz and Winzenbacher, 2017). Point sources consist mainly of discharge of domestic and industrial WWTP effluent. Conventional wastewater treatment removes only a fraction of the micropollutants present in the raw influent, which makes conventional WWTPs important sources for discharge of micropollutants to surface water (Gonzalez-Gil et al., 2017; Ruff et al., 2015; Seitz and Winzenbacher, 2017). The impact of the quality of WWTP effluent on surface water has been 
demonstrated in several studies showing increased micropollutants concentrations along river flows and downstream from WWTPs (Ruff et al., 2015; Tröger et al., 2018). These studies highlight the need to improve current wastewater treatment. However, given the contribution of diffuse sources to the presence of micropollutants in water (Christoffels et al., 2016; Launay et al., 2016), improved micropollutants removal in WWTPs does not fully eliminate these contaminants from our environment.

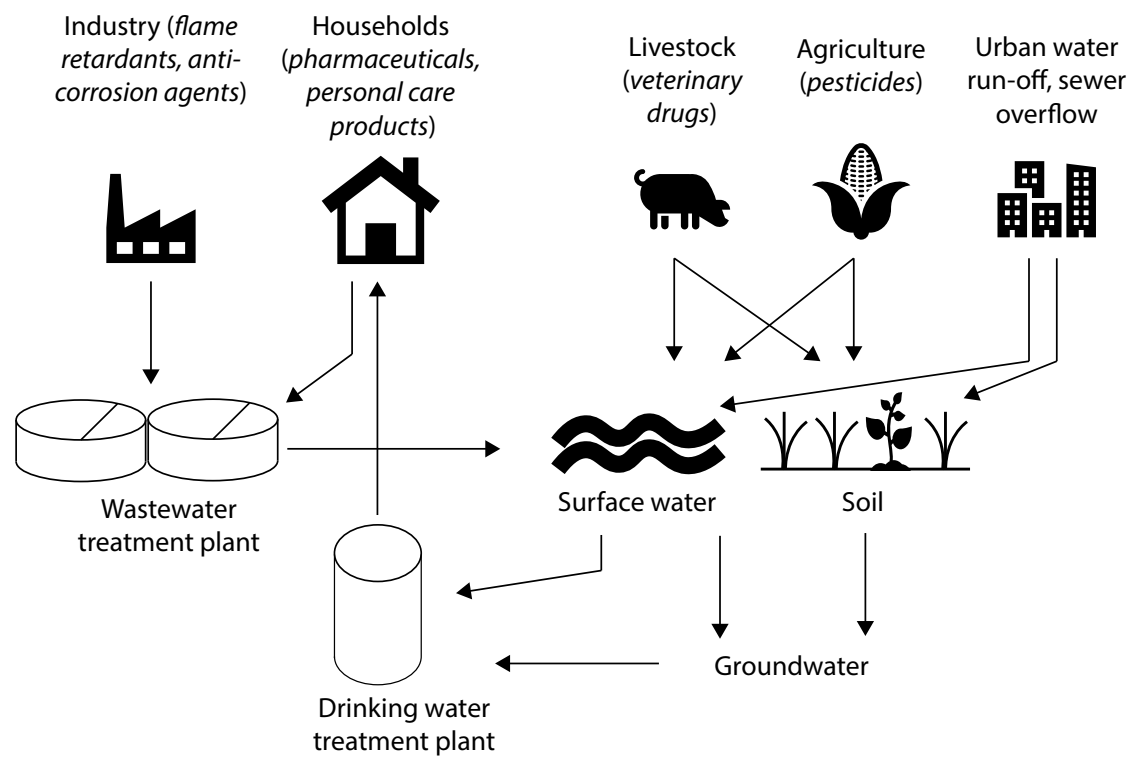

Figure 1.1: Possible sources of micropollutants to the environment and to drinking water.

The presence of micropollutants in the environment has negative impacts to aquatic life and potentially also to human health (Kumerer, 2010; Santos et al., 2010; Sjerps et al., 2016). Negative impacts of micropollutants on aquatic life at environmentally relevant concentrations have been reported, including tissue damage (Triebskorn et al., 2007) and reproduction impairment to fish (Örn et al., 2003; Pawlowski et al., 2004) and reduced activity of crustacea (de Lange et al., 2006). The impacts to human health are more difficult to establish and therefore less well documented (Santos et al., 2010). Some studies conclude that the concentration of micropollutants after drinking water treatment does not pose a risk to human health (Baken et al., 2018; Brunner et al., 2020). However, in the study of Baken et al. (2018) the authors acknowledge that for $54 \%$ of the substances, not enough toxicological or monitoring data is available to make a thorough assessment. Furthermore, the effects of mixtures were not taken into account in their study. 
Negative effects of micropollutants to human health have been demonstrated in vitro using wastewater effluent. These effects were assessed using human cells and include decreased proliferation, increased damage and apoptosis of embryonic kidney cells and overexpression of stress response genes in human intestinal epithelium (Etteieb et al., 2019; Ren et al., 2017). Human exposure to micropollutants through consumption of treated wastewater is currently not a realistic exposure route. However, these results reinforce that the presence of micropollutants in wastewater effluent contributes to deteriorating the quality of surface water used for drinking water production. The contribution of wastewater effluent to the total flow of rivers is expected to increase in the coming years as a result of longer dry periods resulting from climate change (Hofman-Caris et al., 2019; Sjerps et al., 2017). Consequently, micropollutants concentrations in surface water and drinking water are also expected to increase, aggravating the potential risks to the aquatic life and human health.

Determining the extent of the (eco)toxic effects of the presence of micropollutants in water to aquatic life and human health remains challenging for several reasons. Firstly, the enormous amount of chemicals in use, and developed, combined with their degradation products, makes it impractical to study their toxicity on an individual basis. Secondly, mixtures of micropollutants can have different toxic effects than the individual molecules alone. Finally, these negative impacts are more likely to appear as chronic effects, due to long term exposure to low doses, rather than acute effects. However, studies of chronic exposure, are more difficult to conduct and therefore appear in a minority of publications (Jones et al., 2004; Santos et al., 2010). Nevertheless, given the existing evidence of (eco)toxic effects and the remaining knowledge gaps regarding the chronic effects of human and aquatic life exposure to micropollutants, their occurrence in the environment and in drinking water sources should be prevented.

Micropollutants discharge to and maximum concentrations in the environment are not fully regulated in the current European legal framework. The reasons for this legislation gap are several. Firstly, the study of the presence and fate of micropollutants in the environment spans only a few decades, due to recent advances in analytical chemistry. These advances allowed the detection of a larger number of chemicals at lower concentrations. Secondly, the large variety of micropollutants detected in the environment makes it impractical to monitor all contaminants individually. Pesticides are the only class of micropollutants to which a limit in drinking water is established. The Drinking Water Directive (European Council 
Directive 80/778/EEC) sets a limit of $0.1 \mu \mathrm{g} / \mathrm{L}$ for individual pesticides and $0.5 \mu \mathrm{g} / \mathrm{L}$ for the sum of all of them in water intended for human consumption. Yet, the compounds that should be monitored are not specified. Regarding all other micropollutants, a signalling value of $1 \mu \mathrm{g} / \mathrm{L}$ is established by Dutch legislation (Drinkwaterbesluit) for "anthropogenic substances" in water intended for human consumption. Drinking water companies in the Netherlands need to signal when organic contaminants are present in their intake water at concentrations higher than $1 \mu \mathrm{g} / \mathrm{L}$, the so called derogation limit. When this concentration is exceeded, an exemption needs to be requested to the responsible authorities so that the intake water can still be used for drinking water production. A maximum concentration for the contaminant in the intake water can be established based on the nature of the compound and the available information regarding its risks to human health. If the concentration in the intake water exceeds this limit, an alternative source needs to be used. In the last 5 years, the number of micropollutants exceeding the derogation limit in surface water used for drinking water production has increased (Kools et al., 2019), making it challenging for drinking water companies to comply with this regulation.

As our knowledge about micropollutants presence in and removal from water advances, it is expected that the legislation gap will be filled. Micropollutants are nowadays a focus of interest in monitoring programs of surface water (Ruff et al., 2015) and different prioritization schemes have been proposed to ensure these programs target the most relevant contaminants (Brunner et al., 2019; Sjerps et al., 2016).

\subsection{Micropollutants removal in granular activated carbon filters}

Drinking water treatment provides a barrier for human exposure to micropollutants through ingestion. Conventional drinking water treatment is designed to remove suspended solids, organic matter and pathogens from water and improve its odour and taste. This is achieved in a treatment train based on flocculation, separation, disinfection and filtration steps (Stackelberg et al., 2004; Tröger et al., 2020). The chosen technologies and the order in which they are applied depend greatly on the quality of the intake water (Knezev, 2015). More recently, attention is being paid to the efficacy and efficiency of these treatment steps in removing micropollutants. The efficiency of each treatment technology for removing micropollutants is still uncertain, as it depends on type of micropollutant, water matrix properties, and process conditions (Fischer et al., 2019). Studies in full-scale drinking water treatment plants have shown insignificant to significant micropollutants removal after water treatment by flocculation, disinfection (with chlorine, UV or ozonation), sand 
filtration and granular activated carbon (GAC) filtration, depending on the micropollutant (Huerta-Fontela et al., 2011; Stackelberg et al., 2007; Sun et al., 2020; Tröger et al., 2018).

GAC filtration has been applied for decades in industrial and drinking water treatment (Benstoem et al., 2017). GAC filters remove natural organic matter, odour and taste compounds and micropollutants from water, amongst others (Velten et al., 2011a). Also in wastewater treatment, GAC filtration for micropollutants removal has emerged in the last years as a strategy to comply with more stringent effluent discharge regulations foreseen for the near future (Benstoem et al., 2017; Meinel et al., 2015; Östman et al., 2019; Reungoat et al., 2010). Compared to other technologies, GAC filtration offers the advantage of removing a broad range of micropollutants, without producing toxic transformation products, as can be the case for chemical oxidation processes (Gunten, 2018). However, GAC adsorption capacity for micropollutants reduces in the presence of organic matter due to competition for adsorption sites and pore blockage by the organic matter (Kennedy et al., 2015; Zietzschmann et al., 2016b). Furthermore, in case a micropollutant concentration decreases in the filter influent, this micropollutant can desorb from the GAC, as a consequence of a concentration gradient between the GAC and the liquid phase (Corwin and Summers, 2011). Despite these limitations, GAC filtration is commonly seen as one of the main steps responsible for micropollutants removal in drinking water treatment due to its high efficiency in removing micropollutants as long as the adsorption capacity is maintained with frequent GAC reactivations (Stackelberg et al., 2007; Tröger et al., 2020).

In GAC filters, micropollutants are removed from water either via adsorption or via biodegradation by the biomass grown on the GAC surface. These processes are discussed in more detail in the following sections.

\subsubsection{Adsorption}

Adsorption refers to the accumulation of molecules (adsorbates) on the surface of an adsorbent, due to the interaction of these molecules with the adsorbent surface. The type and strength of these interactions depend on characteristics of both adsorbent and adsorbate, as well as on solution conditions, such as $\mathrm{pH}$, temperature and salts concentration (Radovic et al., 1997).

Activated carbon (AC) is a widely used adsorbent, due to its high specific surface area and affinity for a wide range of compounds (Worch, 2012). The high specific surface area of ACs (of order $10^{2}$ to $10^{3} \mathrm{~m}^{2} / \mathrm{g}$ ) is the result of a vast internal network of pores created during the 
AC activation, which can be either thermal or chemical. Thermal activation is carried out using steam or carbon dioxide whereas chemical activation is carried out using chemicals as $\mathrm{H}_{3} \mathrm{PO}_{4}, \mathrm{ZnCl}_{2}, \mathrm{KOH}$ and $\mathrm{K}_{2} \mathrm{CO}_{3}$ (Marsh and Rodriguez-Reinoso, 2006). GAC used in drinking water treatment is produced via thermal activation. Materials often used to produce AC originate from non-renewable (coal) or renewable sources (wood and other types of biomass) (Worch, 2012).

The source material and activation process determine the AC physical and chemical properties (Aktaş and Çeçen, 2006a; de Jonge et al., 1996; Karanfil and Kilduff, 1999). The most relevant properties influencing the AC adsorption capacity are related to its internal surface area, the chemical groups present on this surface and the pore dimensions (Karanfil and Kilduff, 1999). Chemically, the AC internal surface area is described based on its charge and on the most abundant functional groups. Physically, ACs can be characterised based on the surface area and volume for the different pore fractions. The porosity of AC is divided, based on its diameter, in macropores ( $>50 \mathrm{~nm}$ ), mesopores (between 2 and $50 \mathrm{~nm}$ ) and micropores $(<2 \mathrm{~nm}$ ) (Worch, 2012). Micropollutants adsorption is expected to occur mainly in the micropore fraction, where the molecules can interact more closely with the pore walls (Li et al., 2002; Xiaojian et al., 1991). These AC properties determine how much total surface area is available for adsorption, which fraction of the pores is available for micropollutants of different sizes and which interactions can occur between the AC surface and the micropollutants.

The strength of the interactions between the AC surface and micropollutants, combined with the amount of surface area available for adsorption, determine the affinity of the AC towards a micropollutant. The affinity of micropollutants for AC is usually assessed by quantifying the micropollutant partition between the liquid phase and the $\mathrm{AC}$ phase at equilibrium, using different micropollutant:AC ratios at a constant temperature. Adsorption isotherm equations can be applied to the results, generating isotherm coefficients. These coefficients are useful for comparing AC affinity for target adsorbates and predicting AC load at a certain micropollutant concentration. However, in GAC filtration, micropollutant removal is not only determined by affinity towards the GAC, but also by the adsorption rate (Worch, 2012).

The rate limiting step in the adsorption of micropollutants is the diffusion in the stagnant film layer around the GAC particles (film diffusion) and/or inside the particle itself (intraparticle diffusion) (Worch, 2012). Micropollutants diffusion rate is determined mainly by the 
molecule dimensions, GAC pore size and the hydraulic regime in which adsorption takes place (Valderrama et al., 2008). Adsorption kinetics can be modelled to gain insight into which properties of both adsorbate and adsorbent influence adsorption rate and to what extent.

Since during adsorption molecules accumulate onto the GAC surface, the adsorption capacity reduces as adsorption sites become exhausted (Tröger et al., 2020; Ye et al., 2019). Tröger et al. (2020) reported a decrease in micropollutants removal efficiency from $92 \%$ to $34 \%$ after water treatment with 71 months old GAC filters, compared to 12 months old GAC filters. Ye et al. (2019) reported different effects of GAC aging on micropollutants adsorption, depending on the compound. A small fraction (3 out of 17) of the micropollutants tested had a higher affinity for aged GAC, which was attributed to the biofilm grown on the GAC surface. However, most micropollutants had less affinity for aged GAC and $40-60 \%$ of the affinity loss occurred already after 20,930 bed volumes, corresponding to 6 months of use. Once the GAC adsorption capacity drops below threshold levels, GAC often needs to be reactivated.

GAC reactivation consists of a thermal treatment which desorbs and oxidizes adsorbed substances from the GAC surface (Worch, 2012). This process requires high energy input and results in carbon loss and release of pollutants into the atmosphere (Nath and Bhakhar, 2011). The high energy input leads to greenhouse gas emissions. For instance, Mousel et al. (2017) compared ozonation, application of powder activated carbon and GAC filters, as technologies to increase micropollutants removal in wastewater treatment plants. The authors conclude that the latter has the highest energy demand and greenhouse gas emissions per volume of treated water and this demand is due to production of the carbon material, rather than transport and energy required for operation. In a different study where different technologies (ozonation, UV irradiation and GAC filtration) for upgrading a drinking water treatment plant are compared, GAC filtration has the lowest energy demand and carbon footprint. Yet, $80 \%$ of the total energy demand and carbon footprint originate from GAC reactivation (Mo et al., 2018). Developments for reducing the GAC reactivation frequency or for more sustainable regeneration methods will contribute to increase sustainability of GAC filtration, while guaranteeing production of safe drinking water.

\subsubsection{Biodegradation}

Micropollutants biodegradability varies greatly depending on the micropollutant and on the conditions in which it is assessed. For some micropollutants, biodegradation or recalcitrance 
is consistent across studies. For instance, ibuprofen and caffeine are often reported as significantly biodegraded $(>70 \%)$ in wastewater treatment whereas carbamazepine is consistently reported as poorly biodegraded (<40\%) (Grandclément et al., 2017; Luo et al., 2014). For another set of micropollutants, reported biodegradability varies greatly depending on the study. For instance, no or poor $(<25 \%)$ diclofenac biodegradation was observed in several studies with activated sludge (Falås et al., 2016; Fernandez-Fontaina et al., 2014), whereas significant biodegradation could be achieved in specific conditions, such as post-treatment in sequencing batch reactors with attached biomass (Falås et al., 2016) or after biomass adaptation (>400 days) (Suarez et al., 2010).

The poor biodegradability of several micropollutants is related to their complex molecular structures (Cirja et al., 2008) and occurrence at low concentrations (Ehrl et al., 2019). Biodegradation pathways and involved microorganisms are only known for a limited fraction of the micropollutants. This knowledge gap is a consequence of the vast diversity of micropollutants in the environment and the fact that their detection in water has only started in the last decades. For instance, the melamine biodegradation pathway has been described in the early 80's (Jutzi et al., 1982), whereas pyrazole biodegradation has first been reported in 2017 (Bertelkamp et al., 2017). The degradation pathway and microorganisms responsible for it remain unknown.

To estimate the fate of micropollutants and other organic contaminants in (waste)water treatment and in the environment, efforts have been made to predict biodegradability based on the compound molecular structure (Aronson et al., 2006; Tadkaew et al., 2011). Tadkaew et al. (2011) reported a positive correlation between the presence of electron donating groups (e.g. hydroxyl or amine groups) on the molecule and its biodegradability. Similarly, the presence of electron withdrawing groups (e.g. chlorine atoms and amide groups) makes the compound less susceptible to biodegradation. However, it is no surprise that the predictability of these models is limited, due to insufficient and variable experimental data used as input for their calibration (Aronson et al., 2006). Additionally, biodegradability of a compound is highly dependent on the composition of the microbial community and the environmental conditions, which makes it difficult to extract more general conclusions.

Two important aspects of micropollutants biodegradation are discussed below. The first (cometabolism vs. metabolism) is related to the relative concentrations of the micropollutants in relation to other sources of organic carbon and energy. The second is an 
environmental condition related to the availability of electron acceptors for the micropollutants oxidation.

\subsubsection{Cometabolic biodegradation}

Micropollutants are expected to be biodegraded in (waste)water treatment via cometabolism (Tran et al., 2013). Cometabolism is defined as the "transformation of a non-growth substrate in the obligate presence of a growth substrate or another transformable compound" (Dalton and Stirling, 1982). Micropollutants are the non-growth substrates while other compounds present in (waste)water at higher concentrations serve as growth substrates. Metabolism, on the other hand, consists in the degradation of a substrate providing energy to sustain microbial activities, including growth and cell division (Fischer and Majewsky, 2014). At trace concentrations, the energy gained from micropollutants degradation is thought to be insufficient to sustain microbial activities (Tran et al., 2013). Therefore, it is assumed that cometabolism is the main mechanism for micropollutants biodegradation in (waste)water treatment.

Cometabolic degradation is a consequence of the ability of a certain enzyme to degrade different substrates (low substrate specificity) (Criddle, 1993). A classical and relevant example of micropollutants degradation in wastewater treatment is cometabolic degradation by ammonium-monooxygenase, the enzyme involved in nitrification. Cometabolic biodegradation of several micropollutants in nitrifying conditions has been reported (Fernandez-Fontaina et al., 2014; Kumwimba and Meng, 2019; Yu et al., 2018).

\subsubsection{Redox conditions}

Redox conditions play an important role in micropollutants biodegradation (Falås et al., 2013). Several micropollutants are degraded exclusively or at higher rates in oxic conditions (Falås et al., 2013; Suarez et al., 2010). Additionally, as mentioned previously, several micropollutants are biodegraded in nitrifying conditions, i.e., in the presence of both ammonium and oxygen. Nonetheless, studies demonstrate that the combination of oxic and anoxic steps contributes to increasing micropollutants removal and reducing toxicity of wastewater, together with the addition of electron acceptors (Völker et al., 2017, 2016). Moreover, micropollutants biodegradation in sulphate-reducing and methanogenic conditions has also been reported (Alvarino et al., 2014; Gonzalez-Gil et al., 2017; Jia et al., 2019). 


\subsubsection{Synergy between adsorption and biodegradation}

One possible way of developing more sustainable methods to regenerate GAC is by exploring the advantages of micropollutants biodegradation in combination with adsorption. Microorganisms capable of micropollutants degradation can benefit from the presence of GAC in different ways. Micropollutants adsorption allows their accumulation on the GAC surface, resulting in high concentration spots close to the surface and increased contact time between biomass and micropollutants (Çeçen and Aktaş, 2012). Additionally, adsorption can buffer shock loads (Bourneuf et al., 2016; Cha et al., 1998; Li and Moe, 2005), remove degradation products and reduce the effect of toxic or inhibitory compounds that might be present in the water (Çeçen and Aktaş, 2012). Moreover, the rough surface of GAC protects the biofilm from shear stress (Abromaitis et al., 2017). Finally, desorption of previously adsorbed molecules results in a second flux of substrate to the biofilm from the GAC, allowing biomass to be active at higher biofilm depths (Herzberg et al., 2006). As a consequence, GAC filters harbour a more diverse microbial community and remove more micropollutants via biodegradation than other less adsorptive materials, such as anthracite (Greenstein et al., 2018), or anthracite and sand filters (Zhang et al., 2017). The synergy between GAC and microorganisms can therefore favour micropollutants biodegradation compared to non-adsorbent biofilm carrying materials.

Despite the potential of micropollutants removal through biodegradation in GAC filters, little is known about the relevance of this removal process in full-scale applications. Pilotscale studies have demonstrated biodegradation of micropollutants, including benzotriazole and iopromide, in GAC filters (Altmann et al., 2016; Greenstein et al., 2018). However, due to the difficulties in distinguishing adsorption from biodegradation, the relative contribution of both processes to micropollutants removal in GAC filters is to a large extent unknown.

Micropollutants biodegradation in GAC filters benefits their removal through adsorption in several ways. Firstly, micropollutants biodegradation reduces their concentration in the liquid phase, so less adsorption capacity is needed (Ma et al., 2018; Scharf et al., 2010). Secondly, in case of micropollutants desorption from GAC, biodegradation can still remove them from the liquid, preventing their presence in the filter effluent (Herzberg et al., 2005). Finally, the biodegradation of previously adsorbed micropollutants contributes to releasing adsorption sites and, consequently, bioregenerating GAC adsorption capacity (Ma et al., 
2018; Scharf et al., 2010). All these factors combined can extend the life time of the filters and reduce the need for GAC thermal reactivation (Greenstein et al., 2018).

\subsection{Granular activated carbon bioregeneration}

Bioregeneration of GAC is achieved when microbial activity contributes to freeing previously occupied adsorption sites (Figure 1.2). In the absence of biodegradation, a micropollutant adsorbs to the GAC until equilibrium is reached. The ratio between the adsorbed and desorbed states of a certain micropollutant are directly related to its affinity for the GAC and the availability of adsorption sites on the GAC surface. If the micropollutant is biodegraded, its removal from the liquid phase promotes desorption from the GAC due to a gradient in the concentration from the GAC to the liquid. This process results in GAC bioregeneration.

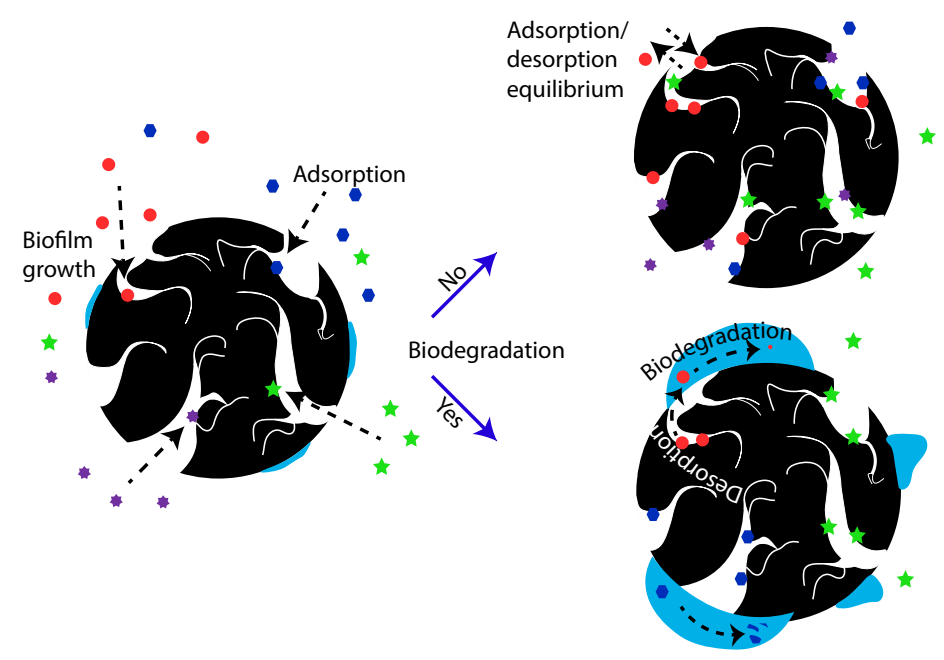

Figure 1.2: Schematic drawing of bioregeneration mechanisms: Biodegradation removes micropollutants from the liquid phase promoting their desorption and consequently GAC bioregeneration.

\subsubsection{Mechanisms of GAC bioregeneration}

Adsorbed micropollutants are spatially separated from microorganisms, hence microorganisms cannot physically reach the micropollutants. This occurs because prokaryotic cells are typically of the order of $0.5 \mu \mathrm{m}$ to $10 \mu \mathrm{m}$ (Alupoaei et al., 2004; de Duve, 1996), whereas pores where adsorption takes place (meso- and micropores) have diameters smaller than $0.05 \mu \mathrm{m}$. In light of this size-exclusion effect, two different bioregeneration mechanisms have been proposed in literature, with the core difference 
being the state (adsorbed or dissolved) of the micropollutant upon its biodegradation (Aktaş and Çeçen, 2007).

One mechanism describes bioregeneration based on the adsorption-desorption equilibrium. Here, it is assumed that the adsorbed fraction of a compound is not available for biodegradation. Biodegradation of micropollutants in the liquid phase can reduce their concentrations to such levels that desorption occurs (Abromaitis et al., 2016). This process continues as long as desorption is not hindered and enough biodegradation takes place. Hence, adsorption reversibility is one of the main limiting factors for bioregeneration according to this mechanism (Nath and Bhakhar, 2011).

Another possible bioregeneration mechanism occurs through the activity of exoenzymes. Exoenzymes can access part of the pores where micropollutants are adsorbed, and subsequently degrade them. Degradation products can desorb to the bulk solution as they usually have less affinity for the GAC, after which they can be degraded by microorganisms (Aktaş and Çeçen, 2007). These two bioregeneration mechanisms are not necessarily contradictory as one or the other can be more relevant depending on the compound being degraded and the pore size fraction being bioregenerated (Sirotkin et al., 2001).

\subsubsection{Quantifying GAC bioregeneration}

Most bioregeneration studies separate adsorption and biodegradation as two different steps (Aktaş and Çeçen, 2009, 2006b; Kew et al., 2016; Toh et al., 2013). The separation of the adsorption and bioregeneration step is often referred to as offline bioregeneration (Aktaş and Çeçen, 2007; Vinitnantharat et al., 2001). With offline bioregeneration, quantification of the removal processes adsorption and biodegradation, and bioregeneration itself is more straight-forward. Using this approach and acclimated biomass, Toh et al. (2013) obtained bioregeneration efficiencies ranging from $47-79 \%$ for GAC loaded with phenol and 33-65\% for GAC loaded with $o$-cresol. Within a certain range, the authors observed higher bioregeneration efficiencies for higher GAC loads (Toh et al., 2013). At higher GAC loads, low affinity adsorption sites are occupied after high affinity adsorption sites are exhausted (Pikaar et al., 2006). Adsorption from low affinity sites is more easily reversible, which reflects on higher bioregeneration efficiencies. Furthermore, bioregeneration of GAC loaded with phenol and other phenolic compounds to comparable extents has been reported, using similar approaches (Aktaş and Çeçen, 2006a; Chan et al., 2018). 
If adsorption and biodegradation occur simultaneously (in situ bioregeneration), quantifying bioregeneration is less straight-forward. Abromaitis et al. (2017) studied GAC bioregeneration in inoculated filters treating influent containing metoprolol. The authors assessed the effects of in situ bioregeneration by comparing adsorption capacity, micropore volume and micropollutant removal after peak load for fresh, bioregenerated and nonbioregenerated GAC. Bioregeneration can also be assessed by analysing non-adsorbable degradation products and performing mass balances (Smolin et al., 2020). Smolin et al. (2020) studied the spontaneous bioregeneration of a GAC filter treating 2-nitrophenol by the indigenous biomass grown in the filter during the study. The authors calculated bioregeneration at determined time intervals based on the nitrogen mass balance in the filter. Bioregeneration was detected in the time intervals when the amount of inorganic nitrogen products of 2-nitrophenol biodegradation were higher than theoretically possible based on the removal in that interval.

The extension of GAC life-time due to biodegradation and occasionally GAC bioregeneration are often quantified by comparing performance of GAC inoculated with specialized biomass with non-inoculated GAC (Jones et al., 1998; Oh et al., 2011, 2012). However, the study of Smolin et al. (2020) and others studies (Jones et al., 1998) have shown that indigenous biomass capable of degrading micropollutants can spontaneouly grow on GAC and consequently promote bioregeneration. Considering these observations and the long time interval between GAC reactivation in drinking water treatment plants (up to several years), a certain degree of spontaneous bioregeneration is expected to occur in fullscale filters.

\subsection{Micropollutants studied in this thesis}

A group of 10 micropollutants was selected for the studies in this thesis (Table 1.1). This selection includes compounds with different applications and physico-chemical properties, which are presented in the experimental chapters according to their relevance to each study. The selection criteria included: 1) their presence in drinking water sources; 2) their incomplete removal during drinking water treatment; 3) the possibility of measuring them at relevant concentrations with the analytical method developed during this thesis, using liquid chromatography and mass spectrometry. 
Table 1.1: Organic micropollutants studied in this thesis.

\begin{tabular}{ll}
\hline Micropollutant & Application \\
\hline Benzotriazole & Corrosion inhibitor ${ }^{1}$ \\
Desphenyl-chloridazon & Biodegradation product of the pesticide chloridazon ${ }^{2}$ \\
Diclofenac & Anti-inflammatory drug $^{3}$ \\
Guanylurea & Biodegradation product of metformin ${ }^{4}$ \\
Hexamethylenetetramine & Industrial chemical with diverse applications ${ }^{5}$ \\
Iopamidol & X-ray contrast agent ${ }^{6}$ \\
Iopromide & X-ray contrast agent ${ }^{7}$ \\
Melamine & Production of resins ${ }^{8}$ \\
Metformin & Antidiabetic drug ${ }^{4}$ \\
Pyrazole & Precursor of pharmaceuticals and pesticides ${ }^{9,10}$ \\
\hline i et al. (2019); ${ }^{2}$ Buttiglieri et al. (2009); ${ }^{3}$ Kosjek et al. (2009); ${ }^{4}$ Briones and Sarmah (2018); ${ }^{5}$ Taghdiri and \\
amani (2013); ${ }^{6}$ Kormos et al. (2010); ${ }^{7}$ Müller et al. (2019); ${ }^{8}$ El-Sayed et al. (2006); ${ }^{9}$ Ganguly and Jacob \\
\end{tabular}

All these compounds have been detected in the intake water used for drinking water production in the Netherlands (Kools et al., 2019; RIWA-Maas, 2016). Diclofenac is included in the Watch List (Directive 2013/39/EU), a list established under the Water Framework Directive (Directive 2000/60/EC), containing pollutants in the aquatic environment that have to be monitored by EU member states. This list aims to determine the risk these compounds pose to the aquatic environment and whether EU environmental quality standards should be set for them. Pyrazole, melamine and hexamethylenetetramine were detected in Dutch surface waters at concentrations exceeding the derogation limit from 2015 to 2018 (Kools et al., 2019; Nieuwenhuizen, 2017; RIWA-Maas, 2016; RIWA-Rijn, 2018, 2016). Additionally, several studies have found these compounds in different water streams (Loos et al., 2009, 2010; Pérez and Barceló, 2007; Ruff et al., 2015; Scheurer et al., 2012; Seitz and Winzenbacher, 2017). Benzotriazole is one of the compounds detected at highest frequency $(>90 \%$ of the samples) and highest concentrations ( $8 \mu \mathrm{g} / \mathrm{L})$ in European surface water (Loos et al., 2009). In the Rhine river, benzotriazole and metformin were listed as two of the main contaminants (Ruff et al., 2015). Metformin is one of the pharmaceuticals most consumed worldwide and guanylurea is its degradation product (Scheurer et al., 2012). Metformin and guanylurea have been found in surface water in Germany and the Netherlands at concentrations up to 5 $\mu \mathrm{g} / \mathrm{L}$ and $28 \mu \mathrm{g} / \mathrm{L}$ respectively, which are among the highest reported for micropollutants in surface water (Scheurer et al., 2012). Desphenyl-chloridazon, the dead-end degradation product of the herbicide chloridazon (Buttiglieri et al., 2009), and melamine were found in a 
water protection area in Germany at concentrations up to $1.2 \mu \mathrm{g} / \mathrm{L}$ and $0.6 \mu \mathrm{g} / \mathrm{L}$ respectively (Seitz and Winzenbacher, 2017). Desphenyl-chloridazon was also detected in several groundwater samples in Europe at concentrations exceeding the legal limit $(0.1 \mu \mathrm{g} / \mathrm{L})$ for pesticides and its degradation products (Loos et al., 2010). X-ray contrast media like iopromide and iopamidol are present in WWTP effluent at concentrations up to $9 \mu \mathrm{g} / \mathrm{L}$ and are frequently found in surface water at concentrations of 2 to $4 \mu \mathrm{g} / \mathrm{L}$ (Pérez and Barceló, 2007). These micropollutants are present in surface water because they are widely used and they are hydrophilic (Sjerps et al., 2016). Hydrophilic micropollutants are the least removed in WWTPs and in natural processes in the environment due to low adsorbability onto sediment, organic matter and sludge (Reemtsma et al., 2016). These properties also make these micropollutants difficult to be removed in the treatment steps of drinking water production.

All compounds studied in this thesis, except for desphenyl-chloridazon, have been reported to be biodegradable under specific conditions (Brunner et al., 2020; El-Sayed et al., 2006; Elizalde-Velázquez and Gómez-Oliván, 2020; Kormos et al., 2010; Langenhoff et al., 2013; Middelhoven and Doesburg, 2007; Schulz et al., 2008; Tisler and Zwiener, 2019; Wagner et al., 2020). However, the extent to which these micropollutants are biodegraded varies greatly, depending on the study. For instance, metformin biodegradation is commonly observed in wastewater treatment, whereas its biodegradation in sand filters used for drinking water production is location-dependent (Marcantonio et al., 2020). Additionally, studies report guanylurea biodegradation in anoxic conditions or in oxic conditions after sludge acclimation (Tisler and Zwiener, 2019), although this micropollutant had been often regarded as a dead-end degradation product of metformin. Similar variability is observed for the other micropollutants studied in this thesis, except for despenhyl-chloridazon, for which no biodegradation has been reported (Altmann et al., 2016; El-Sayed et al., 2006; Knopp et al., 2016; Xu et al., 2013).

\subsection{Knowledge gaps}

The above review of the state of art knowledge on micropollutants removal in GAC filters has indicated various knowledge gaps regarding adsorption of hydrophilic micropollutants onto GAC, their biodegradation in the filters and the resulting GAC bioregeneration.

First, the degree to which several hydrophilic micropollutants adsorb to GAC is unknown. Knowledge regarding the properties of GAC, e.g., pore size distribution and charge, and 
micropollutants, e.g., molecular weight and structure, determining adsorption capacity and rate will contribute to increase their removal with GAC.

Second, micropollutants biodegradation by biomass growing in GAC filters remains to a large extent unknown, mainly due to the difficulties in distinguishing adsorption and biodegradation as removal mechanisms. Most studies reporting micropollutants biodegradation are performed in setups representing wastewater treatment conditions. Knowledge regarding micropollutants biodegradation in drinking water systems is limited and originate mostly from filters with non-adsorbing media, like sand filters. Assessing micropollutants biodegradation by biomass from GAC filters can contribute to determining the potential of this biomass extend the life time of the filters.

Finally, most GAC bioregeneration studies use phenol or phenolic compounds. Few studies report bioregeneration of GAC loaded with micropollutants. One of the key differences between these substrates is the reversibility of their adsorption. Phenol and some phenolic compounds are known to undergo oxidative polymerization on the GAC surface, leading to their irreversible adsorption, which can limit the extent of bioregeneration (Dabrowski et al., 2005). Reversibility of micropollutants adsorption to GAC is to a large extent unknown, as well as the extent to which GAC loaded with micropollutants can be bioregenerated.

\subsection{Objectives and outline of this thesis}

This thesis explores the potential of using microbial degradation of micropollutants to regenerate GAC adsorption capacity and reduce the need for thermal reactivation. To achieve this, the adsorbability and biodegradability of a selection of micropollutants is assessed. Furthermore, adsorption, biodegradation and consequent GAC bioregeneration are studied in more detail using melamine as a model micropollutant. An overview of this thesis is presented in Figure 1.3.

Micropollutants adsorption rate and affinity for 2 types of GAC, a mesoporous chemically activated and a microporous thermally activated GAC are described in Chapter 2. A combination of experimental and modelling work was used to identify the properties of GAC and of micropollutants determining adsorption capacity and rate.

Micropollutants adsorption rate and affinity for 2 types of GAC, a mesoporous chemically activated and a microporous thermally activated GAC are described in Chapter 2. A 
combination of experimental and modelling work was used to identify the properties of GAC and of micropollutants determining adsorption capacity and rate.

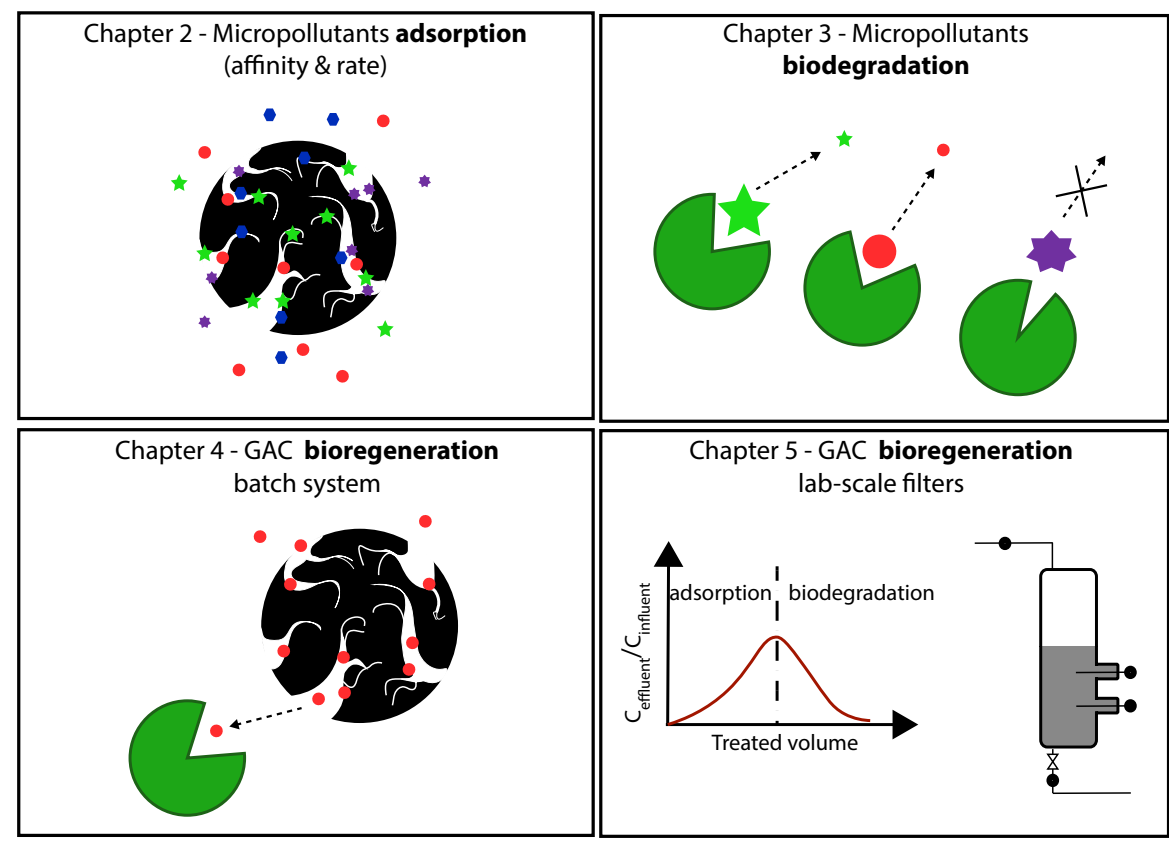

Figure 1.3: Overview of experimental chapters in this thesis.

Biodegradation of micropollutants by indigenous biomass grown in GAC filters from a full scale drinking water treatment plant is described in Chapter 3 to elucidate the potential of this biomass extend the life time of the filters. In this chapter, the effect of GAC aging on adsorption capacity is quantified, as well as the effect of temperature on micropollutants adsorption.

In Chapters 4 and 5 the bioregeneration of GAC was assessed, using melamine as a model micropollutant and melamine degrading biomass. The most favourable conditions for melamine biodegradation were investigated and the limiting factors for (offline) GAC bioregeneration are elucidated in Chapter 4. The extension of filter life-time and simultaneous bioregeneration is crucial to understand the potential of applying this process in practice. This was assessed in lab-scale GAC filters (hapter 5). Adsorption breakthrough curves were modelled to demonstrate the additional value of biodegradation for melamine removal. 
The research findings of the experimental chapters are discussed in relation to the application of bioregeneration in water treatment in Chapter 6. Furthermore, opportunities for future research are identified, which should be carried out in order to support the design of more sustainable water treatment technologies relying on adsorption to GAC. 


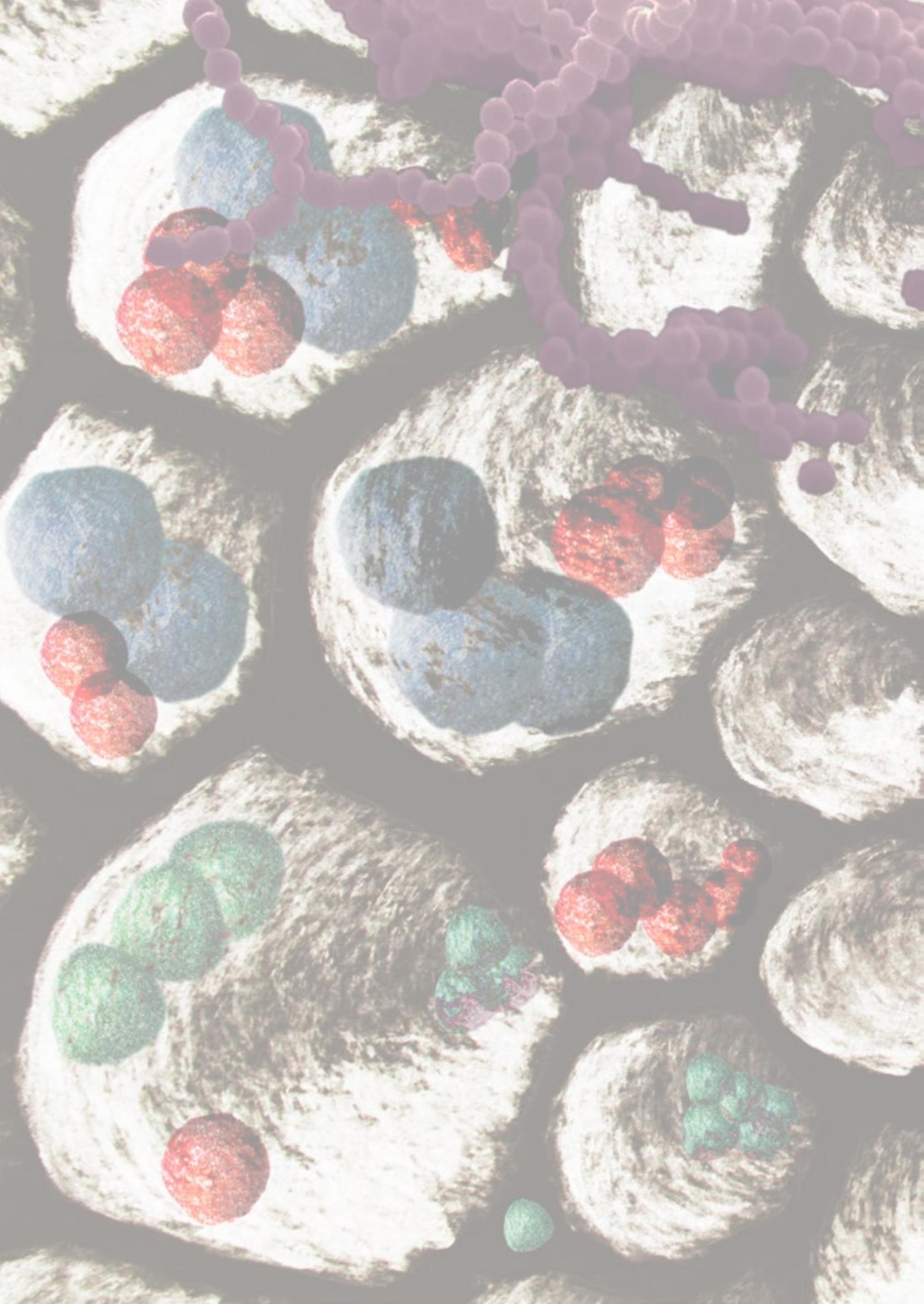




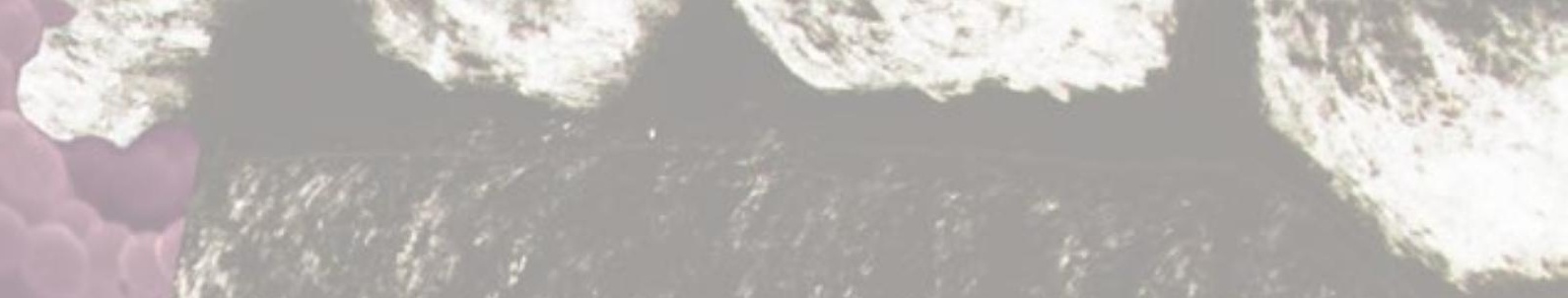

Chapter 2

Diffusion of hydrophilic organic micropollutants in granular activated carbon with different pore sizes

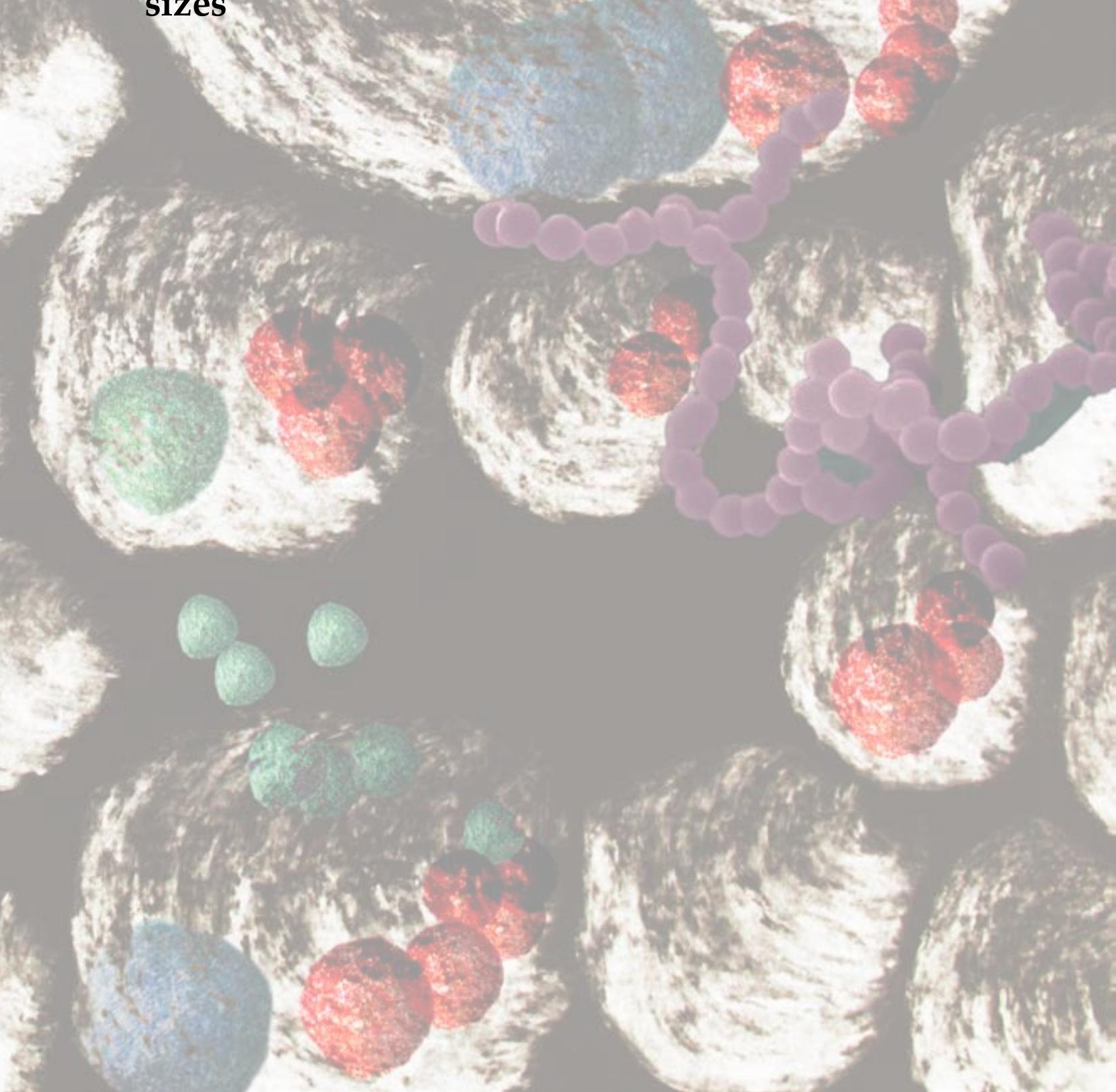




\begin{abstract}
Hydrophilic organic micropollutants are commonly detected in source water used for drinking water production. Effective technologies to remove these micropollutants from water include adsorption onto granular activated carbon in fixed-bed filters. The ratedetermining step in adsorption using activated carbon is usually the adsorbate diffusion inside the porous adsorbent. The presence of mesopores can facilitate diffusion, resulting in higher adsorption rates. We used two different types of granular activated carbon, with and without mesopores, to study the adsorption rate of hydrophilic micropollutants. Furthermore, equilibrium studies were performed to determine the affinity of the selected micropollutants for the activated carbons. A pore diffusion model was applied to the kinetic data to obtain pore diffusion coefficients. We observed that the adsorption rate is influenced by the molecular size of the micropollutant as well as the granular activated carbon pore size.
\end{abstract}

This chapter has been published as:

Piai, L., Dykstra, J.E., Adishakti, M.G., Blokland, M., Langenhoff, A.A.M., van der Wal, A., 2019. Diffusion of hydrophilic organic micropollutants in granular activated carbon with different pore sizes. Water Research. 162, 518-527 


\subsection{Introduction}

Anthropogenic organic micropollutants (OMPs), like pharmaceuticals, industrial chemicals, pesticides, and their transformation products, are often found in surface and groundwater (Christoffels et al., 2016; Loos et al., 2010; Ruff et al., 2015; Scheurer et al., 2009). OMPs may enter the environment via the effluent of wastewater treatment plants and runoff from agricultural land. In the past decades, dozens of OMPs have been detected in water bodies used for drinking water production (Sjerps et al., 2016; ter Laak et al., 2014). Amongst other reasons, this is related to the advance of analytical techniques, which have become more sensitive and selective (Reemtsma et al., 2016; Ternes, 2007). Due to increasing use of anthropogenic organic chemicals, the contamination of surface and drinking water with OMPs will likely increase in the future.

Polar OMPs are less efficiently removed in wastewater treatment plants as well as in drinking water treatment plants (Reemtsma et al., 2016). Screening studies have revealed a relative increase in polar compounds in drinking water samples compared to composition in surface or groundwater (Sjerps et al., 2016). It is often found that the concentration of OMPs in drinking water exceeds $1 \mu \mathrm{g} / \mathrm{L}$ (RIWA-Maas, 2016), which indicates the need for improving current drinking water treatment technologies.

Adsorption onto activated carbon (AC) in fixed-bed filters is one of the main steps for OMP removal during drinking water production from surface water sources (Stackelberg et al., 2007; Ternes, 2007; Ternes et al., 2002). Although, activated carbon is known to be more effective for adsorption of hydrophobic compounds, removal of hydrophilic compounds has been also reported (Nam et al., 2014; Nguyen et al., 2012). Technologies involving adsorption onto AC are cost-effective and normally no transformation products are formed, in contrast to advanced oxidation processes (Gunten, 2018; Miklos et al., 2018). Drawbacks of AC adsorption technologies include the high energy consumption for AC regeneration and slow adsorption kinetics (Worch, 2012). Nevertheless, adsorption onto activated carbon is still regarded as an effective step to remove OMPs from wastewater and drinking water (Katsigiannis et al., 2015).

Activated carbon is a versatile adsorbent due to its affinity for a wide range of compounds and large internal surface area (Nath and Bhakhar, 2011; Worch, 2012). The large surface area of AC originates from the complex internal porous structure, formed during the activation process. To reach the internal surface area where adsorption takes place, the OMPs must diffuse from the solution into the pores. We can distinguish three different 
compartments in which transport takes place: 1) the bulk solution, 2) the thin film layer around the AC particle, and 3) the inside of the particle. The transport in the intra-particle compartment is due to surface and pore diffusion. In surface diffusion, the OMP that is adsorbed onto the AC surface is transported along the carbon internal surface, whereas in pore diffusion the OMP is transported in the liquid phase within the carbon pores. Adsorption kinetics are determined by film, surface and/or pore diffusion, although the relative contributions are dependent on such parameters as mixing regime, adsorbent type and adsorbate properties. It is important to note that in practice it is often difficult to distinguish between surface and pore diffusion (Valderrama et al., 2008).

The rate limiting step during adsorption process of OMPs onto AC can be identified through modelling approaches accounting for the properties of both adsorbate and adsorbent. Lower diffusion rates reduce the efficiency of AC filters due to a more dissipated area between loaded and unloaded zones in the filter bed. Consequently the adsorbate is found in the effluent before the filter bed has been used to its maximum adsorption capacity (Worch, 2012). By modelling the adsorption kinetics of different OMPs onto activated carbon, the apparent diffusion coefficient of the micropollutant in activated carbon can be obtained. This information can then be used to model and optimize removal of OMPs in AC filters.

In this study we assess the affinity of hydrophilic OMPs for two different types of granular activated carbon (GAC) with different pore structure and develop a theoretical model, based on pore diffusion, to describe the adsorption kinetics. Adsorption isotherms and kinetic experiments were used as input for the model to obtain apparent pore diffusion coefficients of 9 OMPs of different sizes using 2 GACs with different pore sizes. The results provide insight in which of the characteristics of OMPs and ACs affect the adsorption rate.

\subsection{Diffusion model}

Several models have been presented in literature to describe adsorption kinetics in porous adsorbents. Some models assume that the transport rate is determined by the adsorption of the adsorbate onto the adsorbent surface. They describe the adsorption rate as a chemical reaction (Ocampo-Pérez et al., 2015). Examples of models using this approach are pseudofirst-order and pseudo-second-order kinetic models as described by Ho et al. and Blanchard et al. (Blanchard et al., 1984; Ho and McKay, 1998). Such models usually fit the data well, but have limited predictive value since the parameters have no physical meaning (Lesage et al., 2010). On the other hand, some models assume that the adsorption rate is determined by 
diffusion. Those are more realistic models based on parameters that are related to the physical and chemical properties of the adsorbent, such as adsorbent porosity and particle radius (Kyriakopoulos and Doulia, 2006). These models take into account film diffusion and/or intra-particle diffusion (Hung and Lin, 2006; Lee and Mckay, 2004; Valderrama et al., 2008).

Intra-particle diffusion can be modelled based on surface and/or pore diffusion, depending which process determines the rate in the system studied. In some studies pore diffusion is described as the dominant transport mechanism (Ocampo-Pérez et al., 2012b), others find a greater contribution from surface diffusion (Ocampo-Pérez et al., 2011, 2010; Zhu et al., 2016), or conclude that both transport processes are relevant (Souza et al., 2017). In general, it is difficult to distinguish between surface and pore diffusion in practice. There are no known properties of the adsorbate and the GAC, nor known conditions of the adsorption process that determine the dominant transport mechanism. Ocampo-Pérez et al. (2015) found that low adsorption capacity of the carbon towards an adsorbate resulted in a higher relative contribution of pore diffusion to the intraparticle diffusion. We assume that pore diffusion will be a relevant diffusion mechanism of hydrophilic compounds in GAC given their lower affinity in comparison to hydrophobic compounds.

We used a pore diffusion model (PDM) to describe the mass transfer of micropollutants towards the internal surface of activated carbon. All OMPs studied in our experiments are hydrophilic, which have in general a lower affinity for AC than hydrophobic micropollutants. Pore diffusion, therefore, was expected to be the dominant mass transfer mechanism. In the model, we consider only pore diffusion for the mass transfer inside the pores. Comparable models have been reported in literature (Ocampo-Pérez et al., 2012a, 2012b; Souza et al., 2017). In the PDM, the AC granules are assumed to be spherical with a constant diameter and homogeneous distribution of adsorption sites. To evaluate the transport and concentration profiles of adsorbate in the pore, $c_{P}(\mu \mathrm{mol} / \mathrm{L})$, and the adsorbent loading, $q_{p}(\mu \mathrm{mol} / \mathrm{g})$, in a spherical particle of porous adsorbent, the following mass balance equation can be set up

$$
\rho_{p} \frac{\partial q_{p}}{\partial t}+\varepsilon_{p} \frac{\partial c_{P}}{\partial t}=d_{p}\left(\frac{\partial^{2} c_{p}}{\partial r^{2}}+\frac{2}{r} \frac{\partial c_{p}}{\partial r}\right)
$$


where $\rho_{p}$ is the activated carbon apparent density $(\mathrm{g} / \mathrm{L}), t$ is time $(\mathrm{s}), \varepsilon_{p}$ is the AC porosity (dimensionless), $d_{p}$ is the pore diffusion coefficient $\left(\mathrm{m}^{2} / \mathrm{s}\right)$ and $r$ is the radius of the adsorbent particle $(\mathrm{m})$. The fitting parameter in the model was $d_{p}$. All other parameters were either calculated, measured or obtained from the AC supplier. AC porosity was calculated based on the ratio between the material apparent density and skeleton density. Details are given in the Supplementary Information (SI) 1 . The particle radius used was the arithmetic mean of the particle size range $\left(3.75 \times 10^{-4} \mathrm{~m}\right)$.

At each point in the pore of the granule we assume a local equilibrium between the OMP concentration in the pore liquid and the adsorbent loading. This equilibrium is described by the Langmuir equation (Langmuir, 1918) given by

$$
q_{e q}=\frac{q_{m} K_{L} c_{e q}}{1+K_{L} c_{e q}}
$$

where $q_{e q}$ is the AC load at equilibrium ( $\left.\mu \mathrm{mol} / \mathrm{g}\right), q_{m}$ is the maximum adsorption capacity of the adsorbent $(\mu \mathrm{mol} / \mathrm{g}), K_{L}$ is the Langmuir constant $(\mathrm{L} / \mu \mathrm{mol})$ and $c_{e q}$ is the micropollutant concentration at equilibrium $(\mu \mathrm{mol} / \mathrm{L})$.

Two boundary conditions are defined:

1) The micropollutant concentration in the bulk solution is equal to the concentration in the AC pores at the boundary with the solution $\left(r=r_{p}\right)$. This condition is based on the assumption that transport limitation due to film diffusion can be neglected.

$$
c_{p}=c_{l} \text { for } r=r_{p}
$$

2) In the center of the particle $(r=0)$ the flux is zero.

$$
\frac{\partial c_{p}}{\partial r}=0 \text { for } r=0
$$

In order to solve Equations 2.1 to 2.4, a mass balance was setup for the batch experiments. Details are given in SI (S2.1).

\subsection{Materials and methods}

\subsubsection{Granular activated carbon}

Two different types of GAC were used in the experiments: AcquaSorb ${ }^{\mathrm{TM}}$ K-CS from Jacobi ${ }^{\circledR}$ (CS), a coconut shell carbon thermally activated and C Gran from Norit ${ }^{\circledR}(C G)$, a wood 
based carbon chemically activated with phosphoric acid. Textural properties were measured with nitrogen adsorption-desorption isotherms at $77 \mathrm{~K}$ using a Micrometrics TriStar 3000, details are given in SI (S2). The ACs differ in micro and mesoporosity (Table 2.1). Micropores constitute almost the entire internal surface area of CS, whereas micropores represent around $68 \%$ of the CG internal surface area and the remaining fraction is formed by mesopores.

Table 2.1: Textural properties of the activated carbons used in this study.

\begin{tabular}{lllllll}
\hline Activation & $\begin{array}{l}\text { Micropore } \\
\text { method }\end{array}$ & $\begin{array}{l}\text { Mesopore } \\
\text { surface area } \\
\left(\mathrm{m}^{2} / \mathrm{g}\right)\end{array}$ & $\begin{array}{l}\text { Mirface area } \\
\left(\mathrm{m}^{2} / \mathrm{g}\right)\end{array}$ & $\begin{array}{l}\text { Micropore } \\
\text { volume }\left(\mathrm{cm}^{3} / \mathrm{g}\right)\end{array}$ & $\begin{array}{l}\text { Mesopore } \\
\text { volume } \\
\left(\mathrm{cm}^{3} / \mathrm{g}\right)\end{array}$ \\
\hline CG & Chemical & & 711 & 334 & 0.32 & 0.43 \\
CS & Steam & 1248 & 48 & 0.51 & 0.04 \\
\hline
\end{tabular}

Granules were sieved to obtain particles with a diameter between $0.5 \mathrm{~mm}$ and $1 \mathrm{~mm}$. After sieving, granules were washed with demineralized water for one hour, dried and stored dried. Before the experiments, GAC was dried overnight at $105{ }^{\circ} \mathrm{C}$, weighed and boiled in demineralized water to remove entrapped air.

\subsubsection{Organic micropollutants}

A mixture of 10 OMPs was used in this study: 1H-benzotriazole (BTA), desphenylchloridazon (DPC), diclofenac (DCF), guanylurea (GNR), hexamethylenetetramine (HMTA), iopamidol (IOP), iopromide (IOPR), melamine (MEL), metformin (MET) and pyrazole (PRZ). DPC was purchased from Santa Cruz Biotechnology and AKOS, IOP was purchased from Sigma Aldrich and AKOS, IOPR was purchased from Sigma Aldrich and Bayer, PYR was purchased from Merck and all other micropollutants were purchased from Sigma Aldrich. Table 2.2 shows their distribution coefficient $\left(\log D_{O W}\right)$, molecular weight and maximum projection area. $\log D_{O W}$ is related to the $\log K_{o w}$ and $\mathrm{p} K_{a}$ of a molecule as described by Equations 2.5 and 2.6 (de Ridder et al., 2010). For neutral molecules, $\log D_{O W}$ equals $\log K_{o w}$. This parameter is often used to indicate hydrophilicity of a molecule, as hydrophilic compounds are characterized by a $\log D_{O W}<3.5$ (Lima et al., 2015), given by

$$
\begin{aligned}
& \text { Acids: } \log D_{O W}=\log K_{o w}-\log \left(1+10^{(\mathrm{pH}-\mathrm{pKa})}\right) \\
& \text { Bases: } \log D_{O W}=\log K_{o w}-\log \left(1+10^{(\mathrm{pKa}-\mathrm{pH})}\right)
\end{aligned}
$$


IOP and IOPR were the largest molecules in the study, followed by diclofenac, as indicated by their molecular weight and maximum projection area. At pH 7.5 GNR and MET are positively charged (Markiewicz et al., 2017a), DCF is negatively charged (de Ridder et al., 2011) and the remaining OMPs are charge-neutral.

These micropollutants were selected due to their relevance for drinking water treatment. They have recently been found in surface and groundwater in Europe at concentrations that might impair drinking water production free of organic contaminants (Alotaibi et al., 2015; Buttiglieri et al., 2009; RIWA-Maas, 2016; Ruff et al., 2015).

Table 2.2: $\log D_{\text {ow }}$, molecular weight, maximum projection area and minimal projection diameter of micropollutants.

\begin{tabular}{lrrrr}
\hline Micropollutant & $\begin{array}{c}\text { Log } D_{\text {OW }} \\
\text { at } \mathrm{pH} 7.0^{1}\end{array}$ & $\begin{array}{l}\text { Molecular } \\
\text { weight } \\
(\mathrm{g} / \mathrm{mol})\end{array}$ & $\begin{array}{l}\text { Maximum } \\
\text { projection area } \\
\left(\mathrm{A}^{2}\right)^{3}\end{array}$ & \multicolumn{2}{l}{$\begin{array}{l}\text { Minimal } \\
\text { projection } \\
\text { diameter (A) }\end{array}$} \\
\hline 1H-benzotriazole & $1.23^{2}$ & 119.13 & 41.23 & 7.04 \\
Desphenyl-chloridazon & $-0.78^{3}$ & 145.55 & 42.76 & 7.82 \\
Diclofenac & $1.37^{4}$ to $3^{5}$ & 296.15 & 69.33 & 9.70 \\
Guanylurea & $-2.06^{3}$ & 102.10 & 38.38 & 6.40 \\
Hexamethylenetetramine & $0.36^{3}$ & 140.19 & 36.39 & 7.34 \\
Iopamidol & $-2.4^{5}$ & 777.09 & 131.62 & 14.14 \\
Iopromide & $-2.1^{5}$ & 791.12 & 129.57 & 14.38 \\
Melamine & $-2.0^{3}$ & 126.12 & 45.30 & 8.00 \\
Metformin & -4 to -3.2 & 129.17 & 43.96 & 7.50 \\
Pyrazole & 0.02 & 68.08 & 26.57 & 5.94 \\
\hline 1: for BTA and PRZ, values reported in literature refer to Log Kow; 2: (Hart et al., 2004); 3: Marvin Scketch \\
(v.16.9.12.0, ChemAxon Ltd.); 4: (Huntscha et al., 2012); 5: Margot et al. (2013); 6: ACD/Labs (V11.02).
\end{tabular}

\subsubsection{Adsorption experiments}

Adsorption isotherm experiments and kinetic experiments were performed by adding a known amount of GAC to a glass serum bottle containing a mixture of 10 OMPs and demineralized water buffered with $10 \mathrm{mM} \mathrm{Na}_{2} \mathrm{HPO}_{4}$ and $10 \mathrm{mM} \mathrm{KH}_{2} \mathrm{PO}_{4}$ at $\mathrm{pH}$ 7.5. All OMPs were present in similar molar concentrations. The bottles were closed with butyl rubber stoppers and wrapped in aluminium foil to prevent OMPs photodegradation. Experiments were conducted in duplicate, at $20^{\circ} \mathrm{C}$ and bottles were mixed at $120 \mathrm{rpm}$ in horizontal position in an orbital shaker. The sample volume subtracted from each bottle was 
$0.5 \mathrm{~mL}$ at every sampling point. Samples were centrifuged for 10 minutes at 10,000 rpm in order to remove GAC particles, diluted when necessary and stored at $-20^{\circ} \mathrm{C}$ before analyses.

\subsubsection{Isotherm experiments}

For isotherm experiments 10 different initial concentrations of micropollutants were used, ranging from 0.6 to $58 \mu \mathrm{M}$ for CG or 1.0 to $120 \mu \mathrm{M}$ for CS. The initial concentration used in the kinetic experiments are within this concentration range (section 3.3.2). GAC mass and total liquid volume were respectively $0.1 \mathrm{~g}$ and $100 \mathrm{~mL}$ for $\mathrm{CG}$, and $0.05 \mathrm{~g}$ and $50 \mathrm{~mL}$ for CS. Equilibrium concentrations were measured at day 8 for CG and day 21 for CS, with the exception of diclofenac, which was measured at day 21 also for CG. AC load at equilibrium was calculated based on the mass balance shown in Equation 2.7

$$
q_{e q}=\frac{\left(c_{0}-c_{e q}\right) * V}{m_{A}}
$$

where $q_{e q}$ is the AC load at equilibrium $(\mu \mathrm{mol} / \mathrm{g}), c_{0}$ is the initial adsorbate concentration $(\mu \mathrm{mol} / \mathrm{L}), c_{e q}$ is the adsorbate concentration at equilibirum $(\mu \mathrm{mol} / \mathrm{L}), V$ is the liquid volume (L) and $m_{A}$ is the adsorbent mass (g).

The Langmuir model was used to fit the experimental data using a nonlinear-optimization method as suggested by Tran et al. (2017) starting from the linearized form of the model given by Equation 2.8

$$
\frac{1}{q_{e q}}=\frac{1}{q_{m} K_{L} c_{e q}}+\frac{1}{q_{m}} .
$$

\subsubsection{Kinetic experiments}

Kinetic experiments were performed with initial OMP concentrations ranging from 34.7 to $60.9 \mu \mathrm{M}, 0.1 \mathrm{~g}$ of GAC and a total liquid volume of $100 \mathrm{~mL}$. Samples were taken at time 0, 2 minutes, 10 minutes, 30 minutes, 1 hour, 5 hours and days 1, 2, 4, 6, 8, 14 and 21. The final volume subtracted from the bottle due to this sampling campaign corresponded to less than $7 \%$ of the total liquid volume.

\subsubsection{Chemical analyses}

Micropollutants were measured using liquid chromatography coupled to high-resolution accurate-mass mass spectrometry (LC-HRAM-MS). The LC consisted of a Ultimate 3000 coupled through a Hesi II electrospray source to a QExactive Orbitrap MS (Thermo Scientific, San Jose, CA, USA). Sample volumes of $50 \mu \mathrm{L}$ were injected onto an Atlantis T3 
column $(100 \mathrm{~mm} \times 3 \mathrm{~mm}, 3 \mu \mathrm{m})$. Micropollutants were separated using gradient elution with a flow of $0.3 \mathrm{~mL} / \mathrm{min}$. Solvents were $(\mathrm{A})$ : water/ammonium formate $2 \mathrm{mM} /$ formic acid $0.016 \%(\mathrm{v} / \mathrm{v})$ and (B): methanol/ammonium formate $2 \mathrm{mM} /$ formic acid $0.016 \%(\mathrm{v} / \mathrm{v})$. All solvents used were UHPLC grade, purchased from Actu-All (The Netherlands). The gradient applied was: 0-2 min linearly increased to $45 \%$ B, 2-8 min linearly increased to $100 \% \mathrm{~B}, 8-14.5 \mathrm{~min}$ stable at $100 \% \mathrm{~B}$, decreased in $0.5 \mathrm{~min}$ to $0 \% \mathrm{~B}$ and stable at this condition until $20 \mathrm{~min}$. The column temperature was maintained at $40^{\circ} \mathrm{C}$. Micropollutants were detected in positive ionisation mode using electrospray. Three different full-scan windows were applied: 60 - 160 for pyrazole, 700 - 800 for iopamidol and iopromide and 60 - 900 for all other micropollutants. The following MS conditions were applied: spray voltage $3.5 \mathrm{kV}$, sheath and sweep gas flow rates 48 and 2 respectively, capillary temperature $256^{\circ} \mathrm{C}$, aux gas heater temperature $413^{\circ} \mathrm{C}$ and resolution 70000. MS was calibrated for each series according to manufacturer protocol using a Pierce ${ }^{\mathrm{TM}}$ LTQ Velos ESI Positive Ion Calibration Solution (Thermo Scientific). Peak identification and quantification was done with Thermo Xcalibur (version 2.2) software.

\subsection{Results and discussion}

\subsubsection{Adsorption isotherms}

Adsorption isotherms were performed to study the affinity of the selected OMPs for both types of activated carbons. All micropollutants showed a higher affinity for CS (Figure 2.1), as shown in Table 2.3 (higher $K_{L}$ values), except for MET and GNR, which showed a higher affinity for CG (Figure 2.2). The affinity of OMPs for CG followed the order: $\mathrm{DCF}>\mathrm{IOPR}>\mathrm{BTA}>\mathrm{IOP}>\mathrm{GNR}>\mathrm{DPC}>\mathrm{MEL}>\mathrm{MET}>\mathrm{PRZ}$. Affinity of OMPs for CS followed the order $\mathrm{DCF}>\mathrm{IOPR}>\mathrm{IOP}>\mathrm{BTA}>\mathrm{MEL}>\mathrm{DPC}>\mathrm{PRZ}>\mathrm{GNR}$. $K_{L}$ values for metformin with $\mathrm{CS}$ and hexamethylenetetramine with both GACs could not be calculated accurately because hardly any adsorption was observed.

The maximum adsorption capacity of CS for some micropollutants can be higher than the $q_{m}$ obtained in our experiments, given that the OMP concentrations applied were too low to reach CS saturation. Nevertheless, together with $K_{L}$, it is justified to use this parameter to describe the distribution of the OMPs between the adsorbed and dissolved phase in the concentration range used in the kinetic experiments.

No OMP adsorption to butyl rubber stoppers was observed in controls without activated carbon (data not shown). 


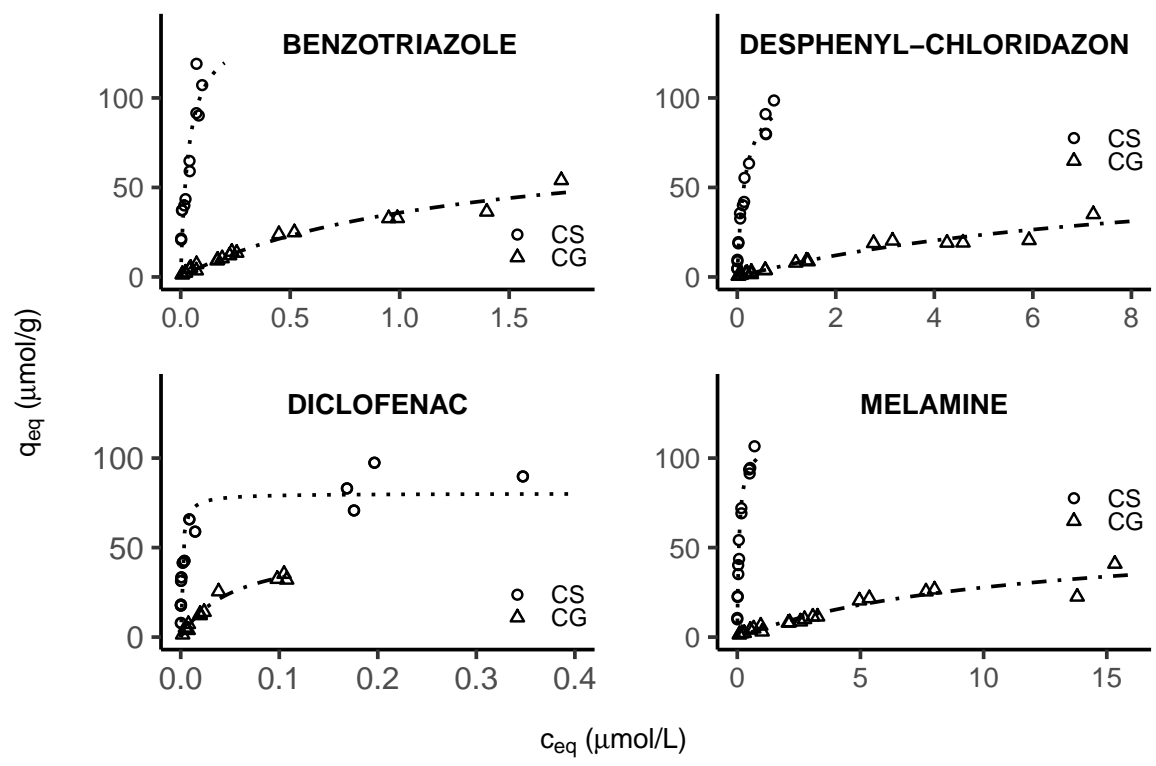

Figure 2.1: Benzotriazole, desphenyl-chloridazon, diclofenac and melamine adsorption isotherms with CG and CS. Lines represent Langmuir model (Equation 2.2).

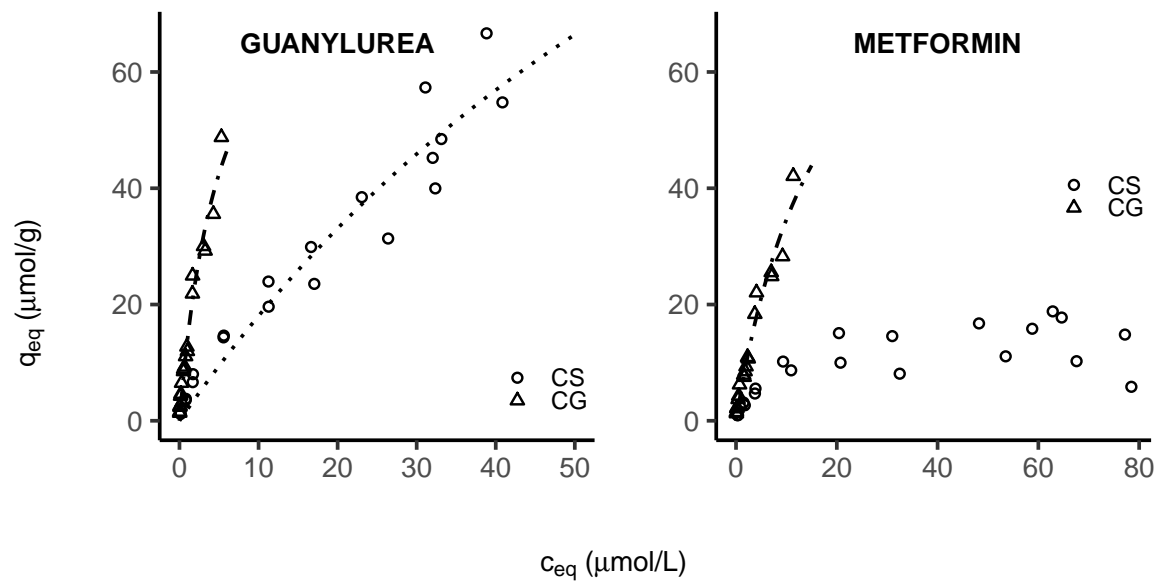

Figure 2.2: Guanylurea and metformin adsorption isotherms with CG and CS. Lines represent Langmuir model (Equation 2.2). 
Table 2.3: Langmuir coefficients of adsorption isotherms and correlation coefficient between experimental data and model prediction. NA: not available.

\begin{tabular}{lrrrrrr}
\hline & \multicolumn{3}{c}{ CG } & \multicolumn{3}{c}{ CS } \\
\hline Micropollutant & \multicolumn{1}{c}{$K_{L}$} & \multicolumn{1}{c}{$q_{m}$} & \multicolumn{1}{l}{$\mathrm{R}^{2}$} & \multicolumn{1}{c}{$K_{L}$} \\
$(\mathrm{~L} / \mu \mathrm{mol})$ & $(\mu \mathrm{mo} / \mathrm{g})$ & & \multicolumn{1}{c}{$\begin{array}{c}q_{m} \\
(\mathrm{~L} / \mu \mathrm{mol})\end{array}$} & $\mathrm{R}^{2}$ \\
\hline BTA & 0.79 & 80.94 & 0.97 & 27.75 & 141.17 & 0.87 \\
DPC & 0.11 & 66.00 & 0.94 & 6.45 & 108.41 & 0.97 \\
DCF & 18.17 & 51.43 & 0.98 & 685.44 & 80.25 & 0.88 \\
GNR & 0.19 & 87.87 & 0.97 & 0.01 & 202.15 & 0.94 \\
IOP & 0.74 & 78.56 & 0.91 & 37.83 & 69.42 & 0.89 \\
IOPR & 1.51 & 97.30 & 0.93 & 69.17 & 74.21 & 0.91 \\
MEL & 0.09 & 60.14 & 0.91 & 12.64 & 109.32 & 0.98 \\
MET & 0.06 & 95.07 & 0.96 & NA & NA & NA \\
PRZ & $1.2 \mathrm{E}-05$ & $4 \mathrm{E} 04$ & 0.91 & 0.07 & 59.53 & 0.91 \\
\hline
\end{tabular}

The higher carbon loading of CS with OMPs for CS is most likely due to the larger micropore surface area of CS (Table 2.1). Stronger adsorption occurs in the micropores (Lu and Sorial, 2004) due to stronger interactions between the adsorbate and the pore walls (Li et al., 2002). A higher adsorption onto microporous AC compared to micro/mesoporous AC has previously been observed by Masson et al. (2016). Pyrazole showed low affinity for CS and almost no adsorption onto CG (Figure 2.3). The low removal with both ACs is most likely related to the small molecule size (MW only 68) in relation to the AC pore size, which reduces the strength of the interactions between the molecule and the pore walls.

GNR, HMTA and MET showed a low affinity for the tested ACs. This may be related to their non-cyclic structure, restricting possible interactions of these micropollutants with the adsorbate. HMTA has a globular structure which restricts the available molecule surface for interaction with the AC. MET and GNR showed poor removal with activated carbon, as reported before (Scheurer et al., 2012). These are the only two positively charged molecules in our study, and both showed a higher affinity for CG than for CS. This is likely due to electrostatic interactions between these molecules and CG. Points of zero charge (pHpzc) values ranging between 3.5 and 4.2 have been reported for CG (Butkovskyi et al., 2018; Villacañas et al., 2006). On the other hand, thermally activated carbons produced from coconut shells, such as CS, have typically $\mathrm{pH}_{\text {pzc }}$ values $>9$ (de Ridder et al., 2013; Dittmar et al., 2018; Largitte and Pasquier, 2016). Therefore, CG most likely has more negatively charged surface groups than CS at neutral $\mathrm{pH}$. Higher adsorption of cationic compounds 
compared with neutral and anionic compounds onto chemically activated carbons was also observed by Alves et al. (2018).

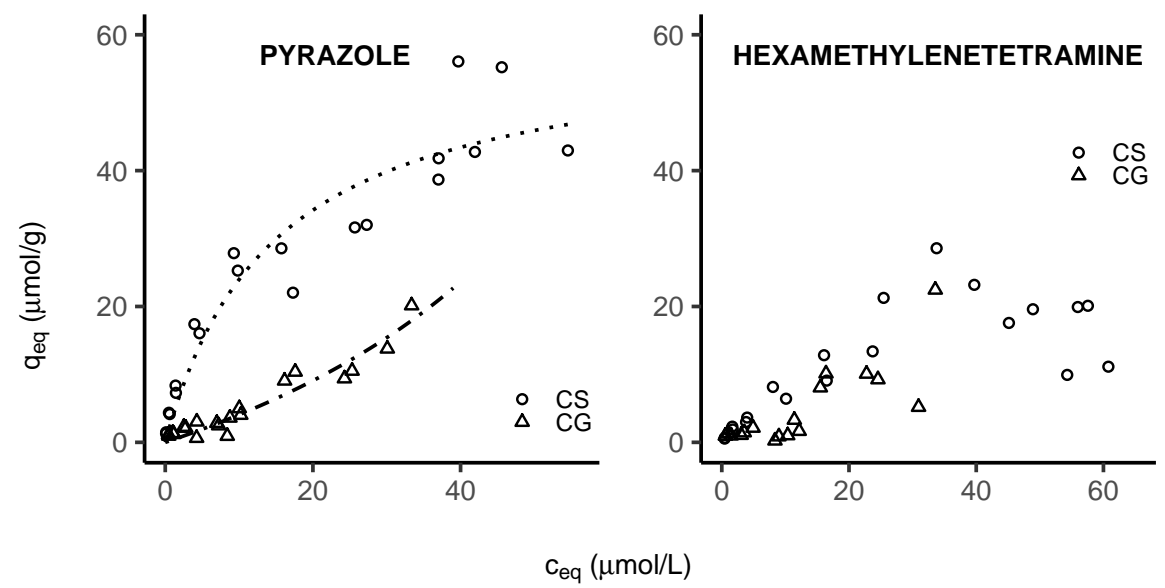

Figure 2.3: Pyrazole and hexamethylenetetramine adsorption isotherms with CG and CS. Lines represent Langmuir model (Equation 2.2).

All micropollutants studied are hydrophilic and no correlation between the degree of hydrophilicity (represented by $\log D_{O W}$ ) and their affinity for the GACs (represented by $K_{L}$ ) was observed. An exception to this observation was DCF, which had the highest $\log D_{O W}$ and highest $K_{L}$. Poor or no correlation between $\log D_{O W}$ and affinity for GAC for hydrophilic compounds is in agreement with what has been previously reported (de Ridder et al., 2010; Kovalova et al., 2013; Nam et al., 2014). For hydrophilic compounds, relevant interactions between adsorbate and activated carbon surface include pi-pi interactions, hydrogen bonds (de Ridder et al., 2010) and electrostatic interactions (Margot et al., 2013). OMPs with aromatic rings (for instance BTA and DCF) can interact with the AC surface via pi-pi interactions. All OMPs from this study are capable of forming H-bonds with the functional groups of the AC and charged molecules (DCF, GNR and MET) are subject to electrostatic attraction or repulsion with charged groups from the AC surface.

The high affinity of DCF and BTA for the ACs obtained in this experiment is in agreement with literature, as those micropollutants are often reported as highly adsorbable on activated carbon (Zietzschmann et al., 2016a, 2014). The Langmuir affinity constant of DCF with both ACs was at least tenfold higher than for the other micropollutants. High affinity of DCF for the ACs may be related to the higher $\log D_{O W}$ of DCF compared to the other 
micropollutants studied (Table 2.2) and to the presence of two aromatic rings in the molecule, increasing the possibilities of pi-pi interactions (Bäuerlein et al., 2012).

IOP and IOPR showed a relatively high affinity for both ACs in our experiments, whereas these micropollutants are often reported as weakly adsorbable (Kennedy et al., 2015; Margot et al., 2013; Rossner et al., 2009; Zietzschmann et al., 2014). The divergence between our results and what is often reported in literature for IOP and IOPR can be explained by the absence of background organic matter in our experiments. The presence of dissolved organic carbon in concentrations as low as $1.5 \mathrm{mg} / \mathrm{L}$ can reduce the adsorption of IOP to GAC by a factor of 7 compared to the adsorption in demineralized water (Ahn et al., 2015). Due to the large size of these micropollutants, their removal with AC is more affected by the presence of background organic matter compared with the smaller compounds (Zietzschmann et al., 2015), either due to pore blockage or competition for adsorption sites. The difference between CG and CS load $\left(q_{e q}\right)$ for IOP and IOPR is smaller than for the other micropollutants (Figure 2.4). This indicates that not all micropore surface area of CS can be occupied by these molecules due to their large size, as predicted based on the molecules diameter (Table 2.2).

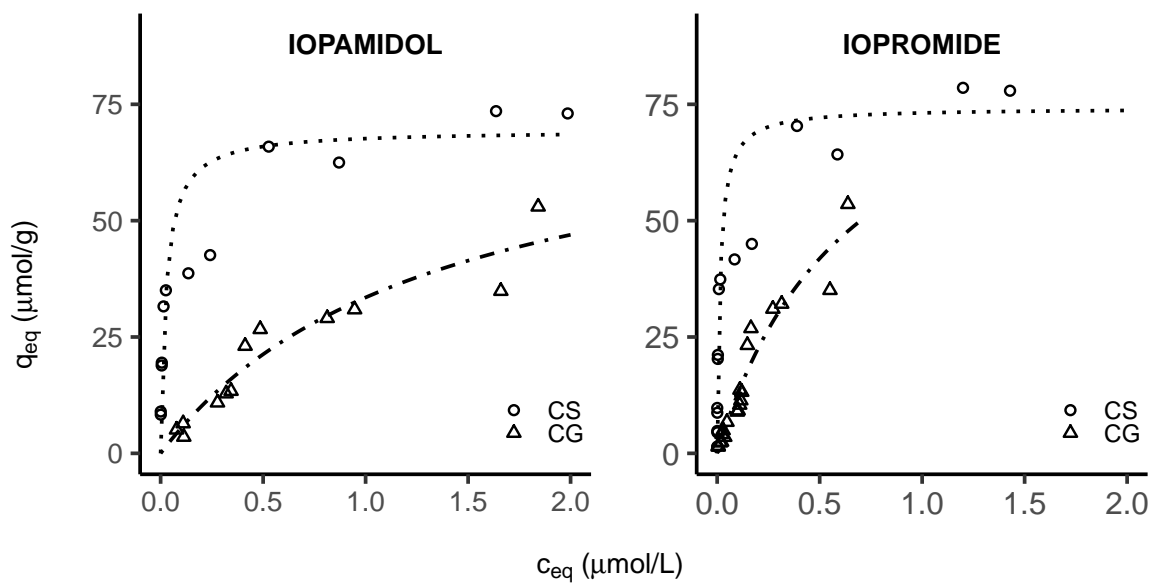

Figure 2.4: Iopamidol and iopromide adsorption isotherms with CG and CS. Lines represent Langmuir model (Equation 2.2).

Possible interactions between the adsorbates in the mixture have not been taken into account when fitting the Langmuir as well as the Pore Diffusion models to our experimental data. We believe this approach can be justified, because based on the projected area of each compound (Table 2.2), at most only $15 \%$ of the GAC surface area available was used for 
adsorption. Moreover, displacement of an adsorbate with lower affinity for the AC by another adsorbate with higher affinity was less than $8 \%$ in the kinetic experiments (at $t>120 \mathrm{~h}$ ), except for the pair guanylurea-CS in one of the replicates (18\% decrease in adsorbed amount between day 8 and day 21). Therefore we conclude that mixtures of OMPs can be used to determine parameters for single solute isotherm and kinetic models.

\subsubsection{Adsorption kinetics of OMPs onto GAC}

In the kinetic adsorption studies, the adsorption rate was related to OMP molecular size and GAC pore size. We observed that equilibrium was reached within $24 \mathrm{~h}$ for the small molecules (BTA, DPC, GNR, MEL, MET, PRZ), whereas for DCF, equilibrium was reached within $24 \mathrm{~h}$ for CG and within $120 \mathrm{~h}$ for CS. For IOP and IOPR, the largest molecules in the study, equilibrium was reached within $50 \mathrm{~h}$ for CG and $300 \mathrm{~h}$ for CS.

Pore diffusion coefficients $\left(d_{p}\right)$ were obtained by fitting the Pore Diffusion model to the kinetic data for the OMP-GAC pairs (Table 2.4). The obtained values of $d_{p}$ are in the same range as previously reported for adsorption with activated carbon (Lesage et al., 2010; Ocampo-Pérez et al., 2012a). The value for $d_{p}$ was not calculated for PRZ with CG since almost no adsorption was observed nor for MET with CS and HMTA with both ACs.

We observed a negative correlation between diffusion coefficients $\left(d_{p}\right)$ and molecular size (Figure S2.2), and a positive correlation between $d_{p}$ and the presence of mesopores in GAC for BTA, DPC, DCF, IOP and IOPR. For these OMPs, the adsorption rate was higher for CG than CS (Figure 2.5, Figure S2.3 and Figure S2.4), due to the presence of mesopores in CG (Table 2.1). For all micropollutants except for IOP and IOPR, the model curve was calculated using the average initial OMP concentration of the experimental duplicates. For IOP and IOPR with CG, the difference between the initial concentration of the duplicates was relatively high and thus two model curves were calculated using the initial concentrations of each duplicate. 
Table 2.4: Fitted pore diffusion coefficient $\left(d_{p}\right)$ of OMPs in the two GACs, OMPs molecular weight (MW) and molecular diffusivities $(D)$ based on Equation 2.10 and GAC tortuosity $(\tau)$ values based on Equation 2.9. NA: not available.

\begin{tabular}{|c|c|c|c|c|c|c|}
\hline & \multicolumn{2}{|c|}{$d_{p}\left(\mathrm{~m}^{2} / \mathrm{s}\right)$} & \multirow{2}{*}{$\begin{array}{c}D\left(\mathrm{~m}^{2} / \mathrm{s}\right) \\
-\end{array}$} & \multirow{2}{*}{$\begin{array}{c}\begin{array}{c}\text { MW } \\
(\mathrm{g} / \mathrm{mol})\end{array} \\
-\end{array}$} & \multicolumn{2}{|c|}{$\tau$} \\
\hline Micropollutant & CG & CS & & & CG & CS \\
\hline BTA & $5.5 * 10^{-10}$ & $3.5 * 10^{-10}$ & $1.1 * 10^{-9}$ & 119.13 & 1.8 & 2.2 \\
\hline DPC & $3.5 * 10^{-10}$ & $3.0 * 10^{-10}$ & $1.2 * 10^{-9}$ & 145.55 & 2.3 & 2.3 \\
\hline DCF & $1.0 * 10^{-10}$ & $6.0 * 10^{-11}$ & $6.8 * 10^{-10}$ & 296.15 & 4.0 & 7.0 \\
\hline GNR & $3.0 * 10^{-10}$ & $3.0 * 10^{-10}$ & $1.5^{*} 10^{-9}$ & 103.00 & 3.3 & 2.9 \\
\hline HMTA & NA & NA & NA & 140.19 & NA & NA \\
\hline IOP & $4.0 * 10^{-11}$ & $2.0 * 10^{-11}$ & $5.0 * 10^{-10}$ & 777.09 & 8.9 & 15.6 \\
\hline IOPR & $5.5 * 10^{-11}$ & $2.5 * 10^{-11}$ & $4.8 * 10^{-10}$ & 791.11 & 7.1 & 12.5 \\
\hline MEL & $1.5^{*} 10^{-10}$ & $3.0 * 10^{-10}$ & $1.2 * 10^{-9}$ & 126.12 & 5.5 & 2.7 \\
\hline MET & $1.5 * 10^{-10}$ & NA & $1.0 * 10^{-9}$ & 129.17 & 4.6 & NA \\
\hline PRZ & NA & $1.5 * 10^{-10}$ & $1.4 * 10^{-9}$ & 68.08 & NA & 3.7 \\
\hline
\end{tabular}
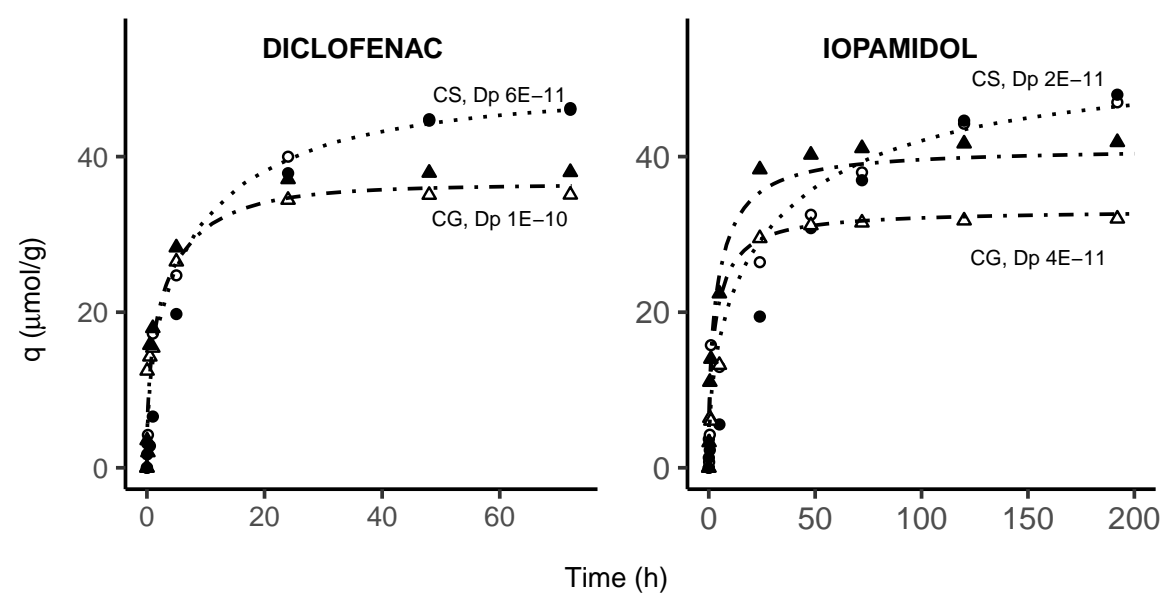

Figure 2.5: Diclofenac and iopamidol AC load in kinetic experiments. Experimental data and fitted pore diffusion model. Hollow and filled symbols represent duplicate batches. For iopamidol on CG, the two lines correspond to the model output with different initial concentrations.

Due to the faster adsorption of some OMPs onto CG than CS, CG load with those OMPs was higher than CS in the beginning of the adsorption process, despite the OMPs higher affinity 
for CS. The period during which CG load was higher than CS was proportional to the OMP molecular size: between 0.5 and $0.75 \mathrm{~h}$ for BTA and DPC, $12 \mathrm{~h}$ for DCF and between 25 and $73 \mathrm{~h}$ for IOP and IOPR. After this period, the CS load with OMPs was higher than CG, as expected based on affinity parameters. This is consistent with the hypothesis that the adsorption rate is limited by intra-particle diffusion and that mesopores facilitate the adsorbate diffusion into the granule (Valderrama et al., 2008). This was also observed by Masson et al. (2016) with activated carbon cloths with different mesopore volumes. Mesoporous ACs have been reported to be more suitable for adsorption of bulky molecules (Liu et al., 2006; Nakagawa et al., 2004; Yuan et al., 2007) compared to microporous ACs, due to size exclusion. The overall affinity of the largest molecules tested (DCF, IOP and IOPR) is higher for CS than for CG. However, as discussed earlier, size exclusion effects of IOP and IOPR with CS can be observed in the isotherms, supporting the hypothesis that diffusion of these micropollutants in the micropores was hindered.

Some OMPs (GNR, MEL, MET and PRZ) showed no correlation between $d_{p}$ and molecule size. PRZ is the smallest molecule in this study, but did not have the highest diffusion coefficient as could be expected based on its molecular weight. Moreover, GNR adsorbed at similar rates onto both ACs whereas MEL adsorbed faster onto CS (Figure 2.6) indicating that the presence of mesopores in CG did not result in a faster adsorption rate of GNR and MEL onto this AC. No correlation between diffusion coefficient and affinity for the AC (represented by $K_{L}$ ) was observed either.

The lack of correlation between $d_{p}$ and molecule size or affinity for the AC shows that properties of the OMP and/or GAC other than pore size also influence diffusion rates, such as AC chemical surface groups, OMP molecular shape, speciation, etc. In this study, it is not possible to distinguish the influence of mesopores from the effect of adsorbent surface chemistry because the used ACs differ in both aspects. A similar conclusion was obtained by Ocampo-Pérez et al. (2012a). In their study, no clear trend was obtained when comparing adsorption rates (given by second-order kinetics model) of compounds with different sizes onto GACs with different porosities. 


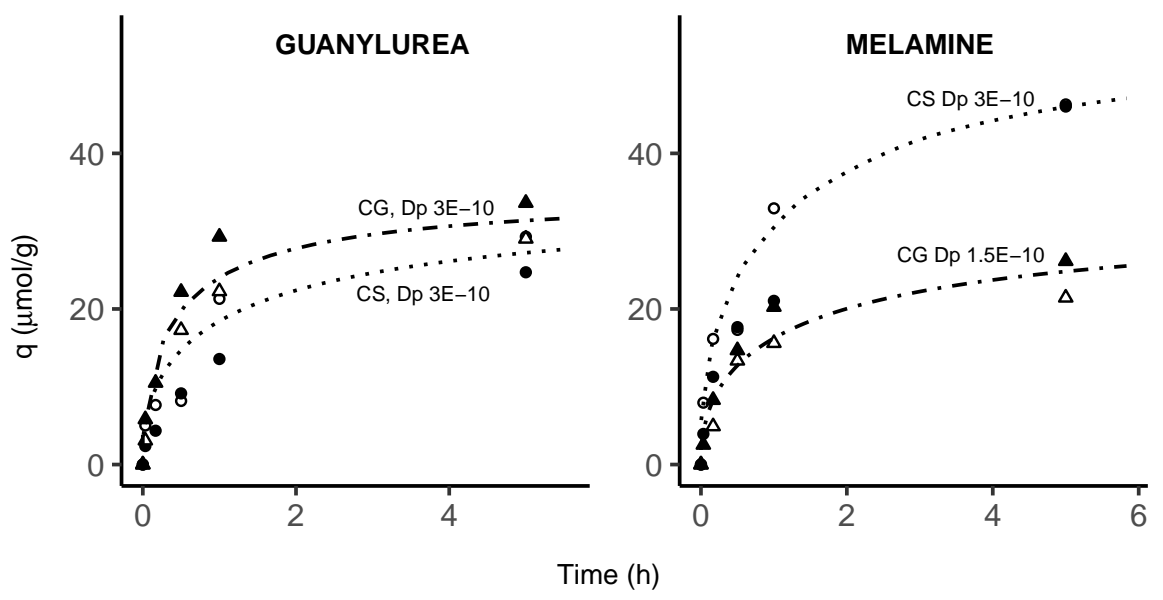

Figure 2.6: Guanylurea and melamine AC load in kinetic experiments. Experimental data and fitted pore diffusion model. Hollow and filled symbols represent duplicate batches.

The model describes the adsorption process after $0.5 \mathrm{~h}$ for the smaller molecules and after $24 \mathrm{~h}$ for the larger molecules. For early stages, the model overestimates the adsorbed amount of OMP. This may indicate that film-diffusion limits adsorption rate at the beginning of the adsorption process. Intra-particle diffusion is less relevant at initial stages since adsorption starts at the outer layers of the AC granules. This has been described by Valderrama et al. (2008) for polycyclic aromatic compounds and activated carbon. In their study with PAHs, the initial steps of the adsorption process could not be represented by models considering intra-particle diffusion as rate limiting step, due to the small thickness of the reacted layer. Our study shows that this is also valid for smaller polar molecules.

\subsubsection{Pore diffusion coefficient}

The pore diffusion coefficient of an adsorbate in $\mathrm{AC}$ is related to the adsorbate aqueous diffusion coefficient $(D)$ as described by Equation 2.9 (Valderrama et al., 2008)

$$
d_{p}=\frac{D \varepsilon_{p}}{\tau}
$$

where $D$ is the molecular diffusivity of the adsorbate $\left(\mathrm{m}^{2} / \mathrm{s}\right)$ and $\tau$ is the AC tortuosity factor (dimensionless), which is a parameter that describes the structure of AC and is used to relate the adsorbate diffusivity in the pore to the diffusivity in free solution.

$D$ values for diclofenac and metformin have been reported in literature as $8.1^{*} 10^{-10} \mathrm{~m}^{2} / \mathrm{s}$ (CidCerón et al., 2016) and $1.23 * 10^{-9} \mathrm{~m}^{2} / \mathrm{s}$ (Mondal et al., 2018), respectively. CS and CG 
tortuosity were calculated based on the reported $D$ values and Equation 2.9. Tortuosity values calculated for CG are between 3 and 5, comparable to values typically reported for AC (R. Ocampo-Pérez et al., 2012a; Worch, 2012). The tortuosity value calculated for CS based on DCF is around 8, which is higher than typically reported values in literature.

When $D$ values obtained experimentally are not available, empirical correlations are applied to calculate the molecular diffusivities. One of the most commonly used correlations is described by Wilke and Chang (Valderrama et al., 2008; Wilke and Chang, 1955; Worch, 2012) according to Equation 2.10

$$
D=\frac{7.4 * 10^{-8}(\Phi M)^{0.5} T}{\eta_{b} V_{A}^{0.6}}
$$

where $\Phi$ is an association parameter that represents the effective molecular weight of water with respect to the diffusion process, and has the value of 2.6, $M$ is the solvent molecular weight $(\mathrm{g} / \mathrm{mol}), T$ is temperature $(\mathrm{K}), \eta_{b}$ is the solution viscosity $(\mathrm{cp})$ and $V_{A}$ is the adsorbate molecular volume $\left(\mathrm{cm}^{3} / \mathrm{mol}\right)$. Values for $V_{A}$ for the OMPs were obtained by ChemSketch (ACD Labs) freeware.

Tortuosity values calculated using the aqueous diffusion obtained with Equation 2.10 are presented in Table 2.4. The calculated values are mostly in the range of values reported for AC except for the largest molecules (DCF, IOP and IOPR). The high tortuosity value obtained for these micropollutants, combined with the adsorption kinetic results, indicates that their diffusion is hindered in the micropores, mainly in the GAC CS, likely due to the relatively large size of the molecules.

Since tortuosity is a property of AC, its values should be constant. However, this parameter is determined based on the adsorption of an adsorbate onto the AC surface, so the interaction between the adsorbate and the AC surface will likely influence the values obtained using experimental data. The variation in tortuosity values can also indicate that the intra-particle diffusion is not only governed by pore diffusion, but surface diffusion might also play a role in determining the adsorption rate. However, since CG and CS tortuosity and pore diffusion coefficients for the OMPs are not known, it is not possible to determine the relative contribution of surface diffusion to the adsorption rate. 


\subsection{Conclusions}

The adsorption rate and affinity of OMPs with two types of activated carbons with different pore sizes was studied to determine the rate limiting step in adsorption. The main findings are as follows:

- The choice for the most suitable GAC for micropollutant removal from drinking water should take into account not only affinity for the target compounds but also GAC pore sizes. GACs with mesopores are desirable to prevent that slow kinetics reduce efficiency of a fixed-bed AC filter.

- Pore diffusion coefficients correlate negatively to adsorbate size for most OMPs and this correlation is stronger for the largest adsorbates.

- Neither molecule size nor adsorption affinity are sufficient to explain the adsorption kinetics of GNL, MER, MET, PRZ.

- Diffusion of the largest molecules is hindered in the GAC micropores, resulting in tortuosity values higher than typically reported for AC.

- Micropore surface area correlates with adsorption affinity between OMP and GAC. However, size-exclusion effects were observed for the largest OMPs iopamidol and iopromide.

- $\log D_{O W}$ is a poor indicator for adsorption affinity for the hydrophilic OMPs studied.

- Adsorption affinity relates to OMP molecular structure: OMPs with cyclic structures adsorb to AC to a larger extent than OMPs with linear or globular structures.

\section{Acknowledgements}

This work was financially supported by Evides Water Company N.V. (Rotterdam, The Netherlands). We also want to thank David de Ridder for his valuable contribution to the discussion of the results, Tomas Haasterecht (Biobased Chemistry and Technology, Wageningen UR) for performing the nitrogen physiosorption analysis and helping with data interpretation, Jordi van Mook for his contribution to the experimental work when working at Evides Water Company N.V., and Marta Wells (Chemistry department, Tennessee Tech University) for providing values of Log $D_{O W}$ data for metformin. 


\section{Supplementary information to Chapter 2}

\section{S2.1 Pore diffusion model}

To solve the set of equations comprised in the model, a mass balance was established for the system: the total amount of micropollutant in the system should correspond, at all times, to the amount in the bulk solution and solid phase (both adsorbed and dissolved in the pore liquid). This mass balance is described by equation S2.1.

$$
c_{\text {total }}=v_{r}\left(1-\varepsilon_{b}\right) c_{l}+\varepsilon_{b} v_{r}\left(\rho_{p} \bar{q}_{p}+\varepsilon_{p} \bar{c}_{P}\right)
$$

where $c_{\text {total }}$ is the total amount of micropollutant in the system $(\mu \mathrm{mol}), v_{r}$ is the total batch volume (liquid volume + adsorbent volume, in L), $\varepsilon_{b}$ is the batch porosity (adsorbent volume / liquid volume, dimensionless), $c_{l}$ is the micropollutant concentration in the bulk solution $(\mu \mathrm{mol} / \mathrm{L}), \rho_{p}$ is the AC apparent density $(\mathrm{g} / \mathrm{L}), \bar{q}_{p}$ is the average adsorbent loading, $\varepsilon_{p}$ is the AC porosity (dimensionless) and $\bar{c}_{P}$ is the average adsorbate pore concentration.

The model was solved as follows: First, the Langmuir isotherm parameters were determined, as described in section 2.3.3.1. Then, Equation 2.1 and the average adsorbent loading $\left(\bar{q}_{p}\right)$ were solved numerically, using the method of lines. This method discretizes the equations in space. We used a semi-implicit Runge-Kutta method to solve the resulting set of ordinary differential equations over time, using Maple. The fitting parameter was the pore diffusion coefficient, $d_{p}$.

AC porosity was calculated based on skeletal density and apparent density. The skeleton density used was that of graphite $\left(2.23 \mathrm{~kg} / \mathrm{m}^{3}\right)$ (Marsh and Rodriguez-Reinoso, 2006) and the apparent density was obtained from the material datasheets (Table S2.1). All other parameters used to solve the model are presented in Table S2.1. 
Table S2.1: Parameters used to solve the pore diffusion model.

\begin{tabular}{lrr}
\hline Parameter & CG & CS \\
\hline$\rho_{p}(\mathrm{~g} / \mathrm{L})$ & 230 & 450 \\
$\varepsilon_{p}(-)$ & 0.88 & 0.77 \\
$\varepsilon_{b}(-)$ & $4.64 * 10^{-3}$ & $2.39 * 10^{-3}$ \\
$r(\mathrm{~m})$ & $3.75 * 10^{-3}$ & $3.75 * 10^{-3}$ \\
$v_{r}(\mathrm{~L})$ & 0.01 & 0.01 \\
\hline
\end{tabular}

\section{S2.2 GAC Textural properties}

A mass of 0.05-0.07 $\mathrm{g}$ of GAC was degassed in vacuum at $200{ }^{\circ} \mathrm{C}$ for 1 hour and cooled before analysis. Micropore surface area and volume were determined with the $\mathrm{t}$-plot method, mesopore surface area and volume was determined with the BJH method. The cumulative pore volume distribution for the mesopore size range ( 2 to $50 \mathrm{~nm}$ width) is presented in Figure S2.1.

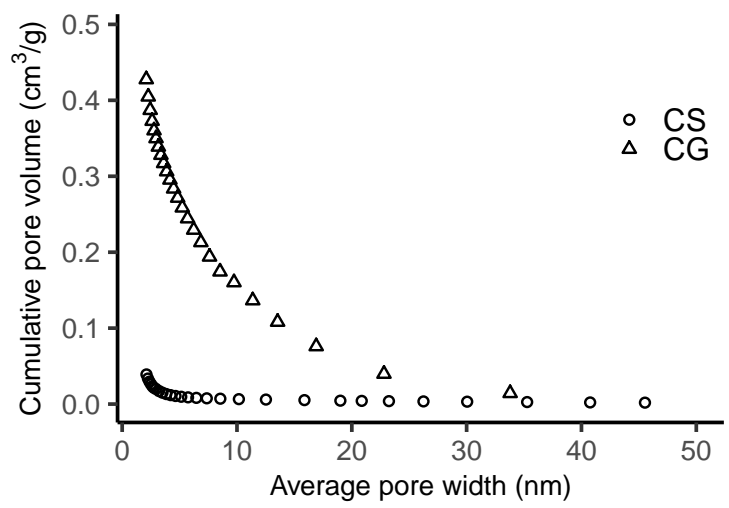

Figure S2.1: Cumulative pore volume distribution of mesopores for CS and CG. 


\section{S2.3 Relationship between pore diffusion coefficient and micropollutant}

\section{parameters}
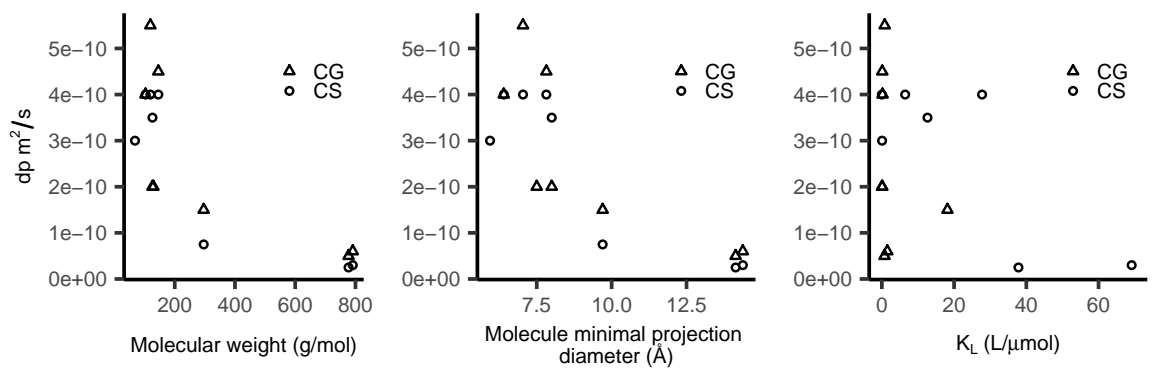

Figure S2.2: Relationship between pore diffusion coefficient $\left(d_{p}\right)$ and: micropollutant molecular weight, molecule minimal projection diameter and Langmuir affinity constant $\left(K_{L}\right)$.

\section{S2.4 Adsorption kinetics}

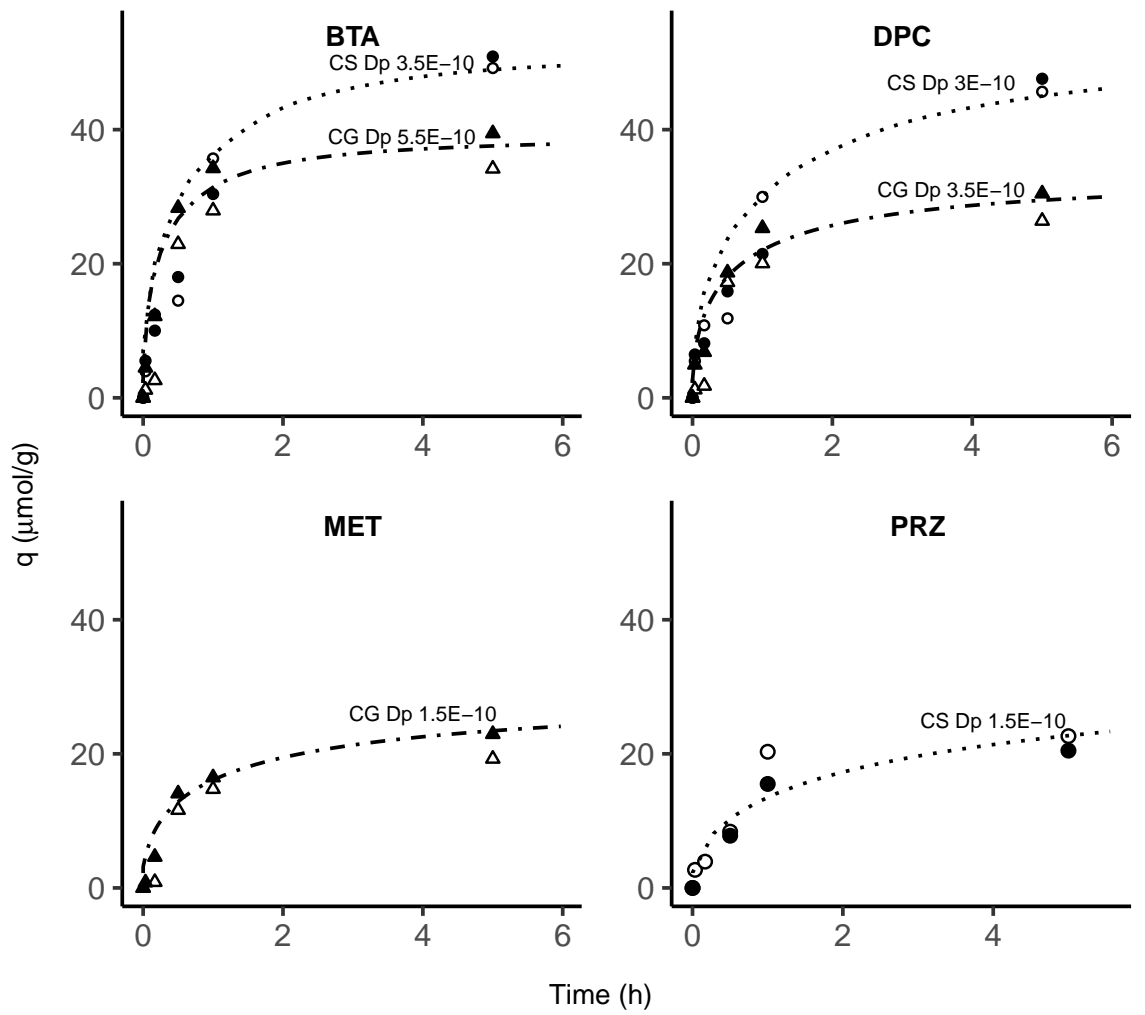

Figure S2.3: AC load in kinetic experiments. Experimental data and fitted pore diffusion model. BTA: benzotriazole, DPC: desphenyl-chloridazon, MET: metformin, PRZ: pyrazole. Hollow and filled symbols represent duplicate batches. 


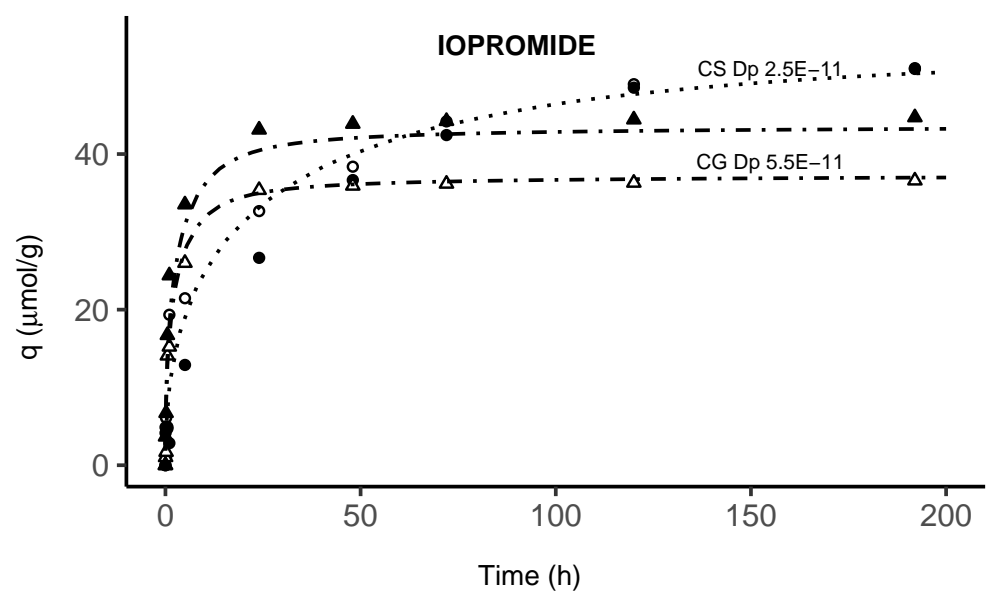

Figure S2.4: Iopromide AC load in kinetic experiments. Experimental data and fitted pore diffusion model. Hollow and filled symbols represent duplicate batches. For adsorption on CG, the two lines correspond to different initial concentrations in the model to accommodate the difference between initial concentration of the replicates. 


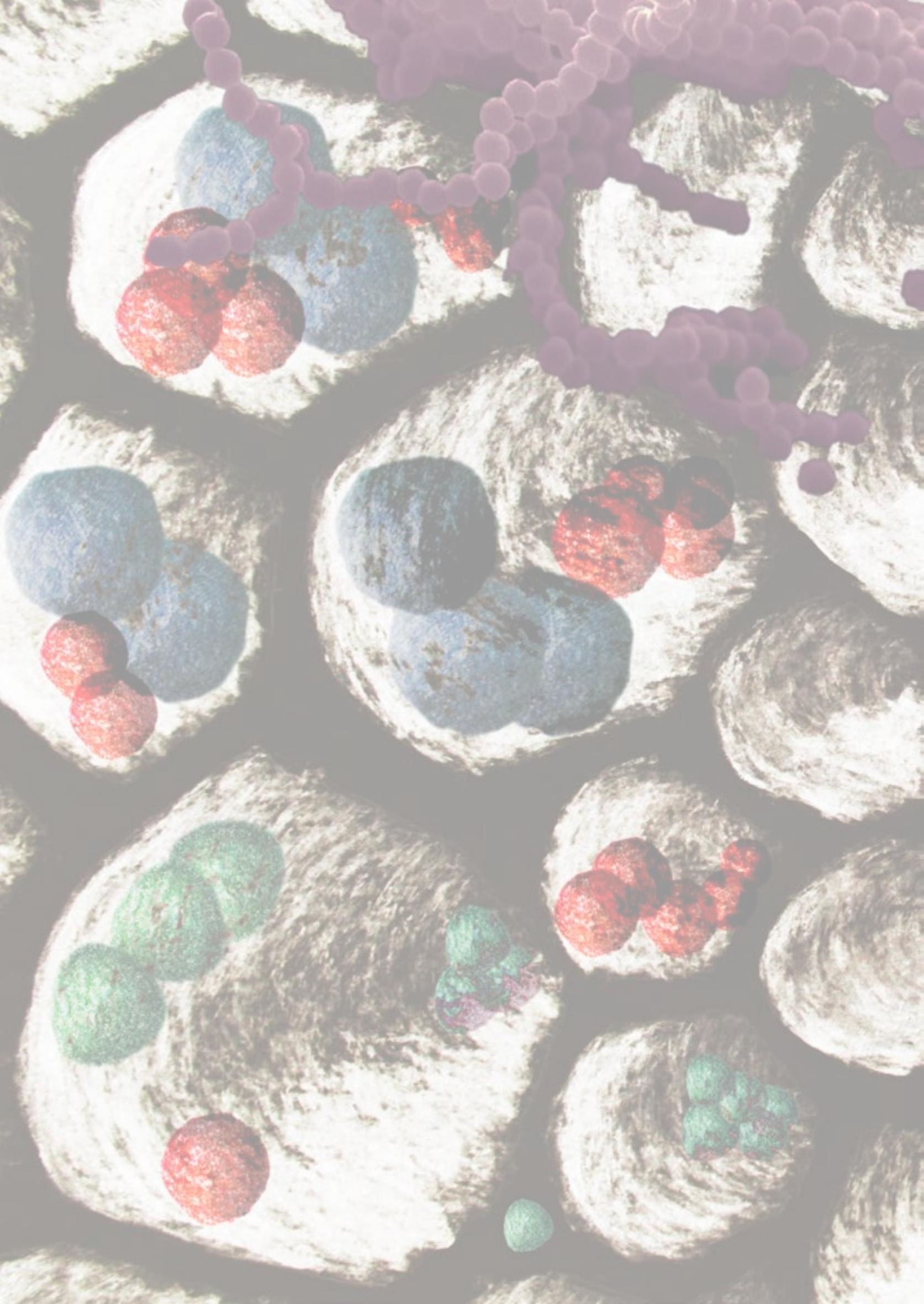





\begin{abstract}
The presence of micropollutants in surface water is a potential threat for the production of high quality and safe drinking water. Adsorption of micropollutants onto granular activated carbon (GAC) in fixed-bed filters is often applied as a polishing step in the production of drinking water. Activated carbon can act as a carrier material for biofilm, hence biodegradation can be an additional removal mechanism for micropollutants in GAC filters. To assess the potential of biofilm to biodegrade micropollutants, it is necessary to distinguish adsorption from biodegradation as a removal mechanism. We performed experiments at $5^{\circ} \mathrm{C}$ and $20^{\circ} \mathrm{C}$ with biologically active and autoclaved GAC to assess the biodegradation of micropollutants by the biofilm grown on the GAC surface. Ten micropollutants were selected as model compounds. Three of them, iopromide, iopamidol and metformin, were biodegraded by the GAC biofilm. Additionally, we observed that temperature can increase or decrease adsorption, depending on the micropollutant studied. Finally, we compared the adsorption capacity of GAC used for more than 100,000 bed volumes and fresh GAC. We demonstrated that used GAC shows a higher adsorption capacity for guanylurea, metformin and hexamethylenetetramine and only a limited reduction in adsorption capacity for diclofenac and benzotriazole compared to fresh GAC.
\end{abstract}

This chapter has been published as:

Piai, L., Blokland, M., van der Wal, A., Langenhoff, A., 2020. Biodegradation and adsorption of micropollutants by biological activated carbon from a drinking water production plant. Journal of Hazardous Materials 388, 122028. 


\subsection{Introduction}

Anthropogenic organic micropollutants have been detected in surface water worldwide (Christoffels et al., 2016; Reungoat et al., 2010; Starling et al., 2019), threatening production of safe drinking water. Micropollutants consist of a wide range of compounds, such as pharmaceuticals, industrial chemicals, pesticides, as well as their transformation products. These compounds often enter into the environment via the effluent of a wastewater treatment plant (WWTP) and diffuse sources, such as stormwater runoff, sewer leakage and sewer overflow (Han et al., 2019). An unknown fraction of micropollutants in the environment is adsorbed to sediments or organic matter, or transformed via biotic and abiotic reactions. The fraction that is not removed, can end up in the feed water used for drinking water production (Mompelat et al., 2009).

Micropollutants are partially removed in a drinking water treatment plant (DWTP) from surface water sources by adsorption onto granular activated carbon (GAC) in fixed-bed filters (Stackelberg et al., 2007; Ternes, 2007; Ternes et al., 2002). GAC has also been applied at pilot scale in filters and fluidized bed reactors for removing micropollutants from wastewater (Altmann et al., 2016; Guillossou et al., 2019; Mailler et al., 2016). Apart from adsorbing the micropollutants, GAC also functions as a carrier material for biofilm. As a result, micropollutants are concentrated on the GAC surface, which results in an increased contact time between the compounds and microorganisms (Çeçen and Aktaş, 2012), and higher likelihood of biodegradation. Biodegradation is therefore an alternative pathway for removing micropollutants in GAC applications. The extent to which a micropollutant will be adsorbed to GAC and eventually be biodegraded depends on the reversibility of adsorption and on the potential of the biofilm to biodegrade the micropollutant (Abromaitis et al., 2016).

Distinguishing between adsorption and biodegradation as removal mechanisms in GAC filters is required in order to assess the potential of the biofilm in these filters to biodegrade micropollutants. Furthermore, this knowledge contributes in determining limiting factors for micropollutants biodegradation in the current GAC filters. In adsorption studies with fresh GAC, the adsorbed amount of a certain adsorbate is calculated based on the change in concentration in the liquid phase, assuming that adsorption is the only removal mechanism (Worch, 2012). When experimenting with biologically active GAC (bGAC), the adsorbate can also be biodegraded, apart from adsorbed. However, inhibiting biodegradation by applying GAC treatments, such as autoclaving, or adding chemicals to the solution can also 
affect adsorption to an unknown extent. For instance, sodium azide, is commonly used as biodegradation inhibitor in adsorption experiments (Shimabuku et al., 2017b; Ye et al., 2019). However, sodium azide affects adsorption, as demonstrated by Klimenko et al. (2010) who studied the influence of this chemical on the removal of fulvic acids by AC. The authors concluded that the sodium azide did not fully inhibit biological activity and also that it interacted with the AC surface thus changing its adsorption properties. In addition to chemically affected AC, autoclaved used AC might have different adsorption properties compared with non-autoclaved $\mathrm{AC}$, because autoclaving can potentially alter the biofilm formed on the AC surface and the organic matter previously adsorbed to it, thus affecting adsorption of micropollutants.

Experiments with bGAC at relatively low and high temperatures are an alternative control strategy to distinguish between adsorption and biodegradation for micropollutants removal. Increasing temperatures up to $42^{\circ} \mathrm{C}$ increases microbial activity (Lengeler et al., 1999). The effect of temperature on adsorption, however, is less predictable. Temperature can have an effect on adsorption rate and on the overall adsorption at equilibrium conditions. Increasing temperatures increase adsorption rate due to a faster adsorbate diffusion (Taghdiri and Zamani, 2013) but the overall adsorption capacity can be either increased or reduced (Moreno-Castilla, 2004), depending on whether the adsorption is endothermic or exothermic (Al-Degs et al., 2008; Gupta et al., 2011; Wang and Li, 2013).

Given the difficulty of distinguishing adsorption and biodegradation as removal processes, few studies have assessed the micropollutants biodegradation capacity of biomass growing in GAC filters used in DWTP (Altmann et al., 2016; Zhang et al., 2017). In contrast, biodegradation of micropollutants by activated sludge from WWTP is well documented in literature (Besha et al., 2017; Grandclément et al., 2017). Our goal is to investigate if the biomass growing in GAC filters is capable of degrading a selection of micropollutants relevant for drinking water. We studied the removal of micropollutants with activated carbon that originates from a drinking water production location. Experiments with biologically active or autoclaved GAC at two different temperatures were performed to distinguish removal due to adsorption and biodegradation or adsorption only. 


\subsection{Materials and methods}

\subsubsection{Organic micropollutants}

A mixture of 10 organic micropollutants was used in this study: benzotriazole, desphenylchloridazon, diclofenac, guanylurea, hexamethylenetetramine, iopamidol, iopromide, melamine, metformin and pyrazole. These selected micropollutants have been found in surface water in Europe in concentrations ranging from 0.1 to $6 \mu \mathrm{g} / \mathrm{L}$, and are considered relevant in drinking water treatment (Buttiglieri et al., 2009; Kiss and Fries, 2009; RIWAMaas, 2016; Ruff et al., 2015). Desphenyl-chloridazon and iopamidol were purchased from AKOS, iopromide was purchased from Bayer, pyrazole was purchased from Merck and all other micropollutants were purchased from Sigma Aldrich. All micropollutants have a neutral charge at $\mathrm{pH} 7.5$, except for guanylurea and metformin which are positively charged (Markiewicz et al., 2017a) and diclofenac which is negatively charged (de Ridder et al., 2011). This mixture consists of hydrophilic micropollutants of different molecular weights (Table 3.1).

Table 3.1: Micropollutants used in this study, their $\log D_{O W}$ and molecular weight.

\begin{tabular}{lrr}
\hline Micropollutant & $\log D_{O W}$ at $\mathrm{pH} 7.0^{1}$ & Molecular weight $(\mathrm{g} / \mathrm{mol})$ \\
\hline Benzotriazole & $1.23^{2}$ & 119 \\
Desphenyl-chloridazon & $-0.78^{3}$ & 146 \\
Diclofenac & $1.37^{4}$ to $3^{5}$ & 296 \\
Guanylurea & $-2.06^{3}$ & 102 \\
Hexamethylenetetramine & $0.36^{3}$ & 140 \\
Iopamidol & $-2.4^{5}$ & 777 \\
Iopromide & $-2.1^{5}$ & 791 \\
Melamine & $-2.0^{3}$ & 126 \\
Metformin & -4 to $-3.2^{6}$ & 129 \\
Pyrazole & 0.02 & 68 \\
\hline
\end{tabular}

1: for benzotriazole and pyrazole, values reported in literature refer to Log Kow; 2: (Hart et al., 2004); 3: Marvin Scketch (v.16.9.12.0, ChemAxon Ltd.); 4: (Huntscha et al., 2012); 5: Margot et al. (2013); 6: ACD/Labs (V11.02).

\subsubsection{Micropollutants biodegradation by biologically active GAC}

To assess the potential of biofilm growing in GAC filters to degrade the selected micropollutants, a batch experiment with used GAC, from a DWTP, was performed. The filter where the used GAC (Filtrasorb TL-830, Chemviron ${ }^{\circledR}$ ) was collected had been in operation for more than 100,000 bed volumes by Evides Water Company (location Kralingen, the Netherlands). This material will be referred to as bGAC. The bGAC worked 
both as adsorbent and inoculum. To supply microorganisms with additional carbon source similar to what is available in the GAC filters, the culture media was prepared using GAC filter influent, obtained from the same DWTP as the bGAC, and demineralized water, in a 1:1 ratio. Concentrated nutrient solutions were diluted in this matrix. Demineralized water was used together with GAC filter influent to avoid high salt concentration in the culture media, which could inhibit microbiological activity. The composition of the concentrated nutrient solutions is given in Table S3.1 and GAC filter influent quality parameters are given in Table S3.2. Additional inoculum was prepared as follows: $15 \mathrm{~g}$ of used GAC was mixed with $20 \mathrm{~mL}$ of culture media and vortexed for 5 seconds. This procedure was repeated several times to obtain biomass in the liquid phase, which was used as additional inoculum.

A mass of $0.26 \mathrm{~g}$ of bGAC wet weight, corresponding to approx. $0.1 \mathrm{~g}$ dry weight, was used to inoculate $250 \mathrm{~mL}$ serum bottles. The bottles contained $10 \mathrm{~mL}$ of additional inoculum, micropollutants and culture media in a total liquid volume of $100 \mathrm{~mL}$. All micropollutants were added in similar molar concentrations and three different initial concentrations were used, approximately $25 \mu \mathrm{M}, 45 \mu \mathrm{M}$ and $69 \mu \mathrm{M}$ (ranging from 1.7 to $54.6 \mathrm{mg} / \mathrm{L}$ depending on the micropollutant). Initial micropollutants concentrations were measured after spiking the bottles. Therefore, in case these micropollutants were already present in the GAC filter influent used to prepare the media, the initial concentration was the sum of the spiked and the already present micropollutants. The bottles were closed with butyl rubber stoppers and aluminium crimp caps, wrapped in aluminium foil to prevent micropollutants photodegradation and cultivated at either $5^{\circ} \mathrm{C}$ or $20^{\circ} \mathrm{C}$, mixed at $120 \mathrm{rpm}$. Triplicates were run for each condition (Figure 3.1). Samples were taken on days 0, 9, 21, 33, 44 and 54. Samples were centrifuged for $10 \mathrm{~min}$ at $10000 \mathrm{rpm}$, the supernatant was transferred to a clean tube and centrifuged again. After that, the supernatant was diluted and frozen until analysis. Results up to the $54^{\text {th }}$ day were sufficient to test our hypothesis and draw conclusions and the experiment was stopped.

\subsubsection{Micropollutants adsorption onto autoclaved used GAC}

The effect of temperature on micropollutants adsorption to GAC was assessed in a test with autoclaved used GAC. This experiment was similar to the one described in 2.2 with the following differences: used GAC and media were autoclaved before being used and no additional inoculum was added to the bottles. Since no biodegradation was expected in experiments with autoclaved GAC, we expected little variation between replicates, which indeed was observed in our data. Therefore, only duplicates were run. This experiment was 
done with one initial concentration of micropollutants, approximately $69 \mu \mathrm{M}$ (Figure 3.1). Bottles were cultivated in the same conditions as in the experiment described in 2.2 and samples were taken on days $0,9,21,32,44$ and 53 .

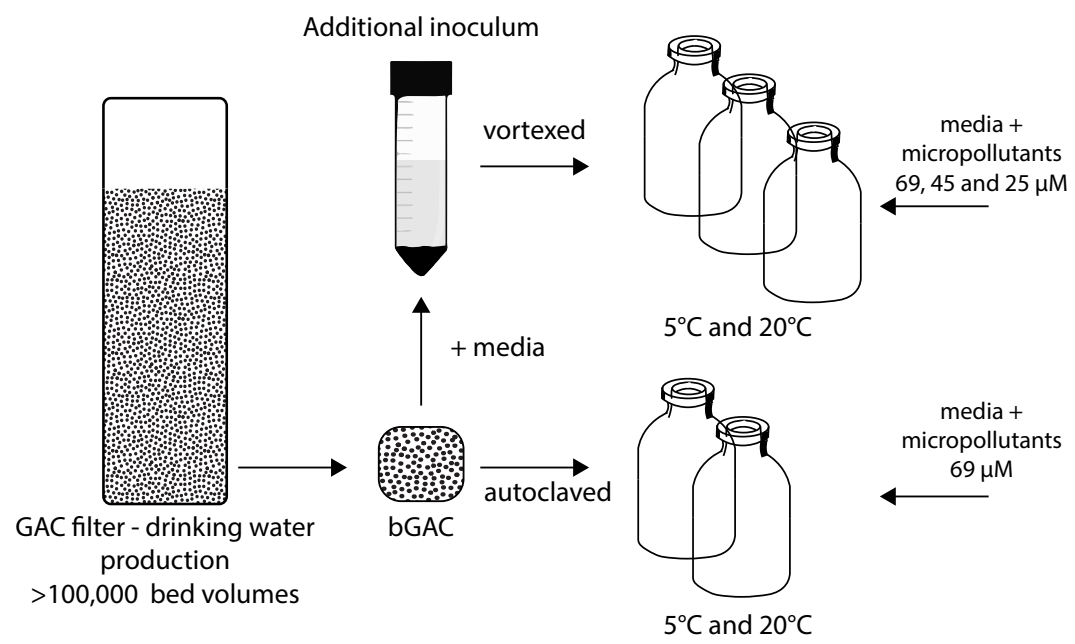

Figure 3.1: Schematic representation of experiments with used GAC.

\subsubsection{Micropollutants adsorption onto fresh GAC}

To compare the adsorption capacity of used GAC and fresh GAC, an additional adsorption experiment was performed. Experimental conditions were similar as described in 2.2 and 2.3, except that: 1) fresh GAC (Filtrasorb TL-830, Chemviron ${ }^{\circledR}$ ) was used instead of used GAC; 2 ) only demi-water was used in the preparation of the media instead of a mixture of demi-water and GAC filter influent; 3 ) one initial concentration of micropollutants was used $(62 \mu \mathrm{M})$ instead of 3 initial concentrations. Bottles were kept at $5^{\circ} \mathrm{C}$ and mixed at $120 \mathrm{rpm}$. Samples were taken on days 0 and 9. Previous studies showed this time interval to be sufficient for fresh GAC to reach near equilibrium conditions for adsorption of the selected micropollutants (Piai et al., 2019).

\subsubsection{Chemical analyses}

Micropollutants were measured using liquid chromatography coupled to high-resolution accurate-mass mass spectrometry, according to the method described in Piai et al. (2019). In short, compounds were separated using an Atlantis T3 column and a gradient based on water/ammonium formate $2 \mathrm{mM} /$ formic acid $0.016 \%(\mathrm{v} / \mathrm{v})$ and methanol/ammonium formate $2 \mathrm{mM} /$ formic acid $0.016 \%(\mathrm{v} / \mathrm{v})$. Mass spectrometric analysis was performed in positive 
electrospray ionisation mode on a Thermo QExactive MS in full-scan mode (resolution=70000).

\subsection{Results and discussion}

\subsubsection{Distinguishing micropollutants biodegradation and adsorption}

We assessed whether biofilm from GAC filters can biodegrade a selection of micropollutants by performing experiments with bGAC and autoclaved GAC at different temperatures. We assumed that at low temperature, the removal of the micropollutant was mainly due to adsorption, thus serving as control. Since temperature can also affect adsorption, experiments with autoclaved GAC were performed in order to assess the extent of the effect of temperature on adsorption.

Until day 9, the removal of iopromide with bGAC was similar at $5^{\circ} \mathrm{C}$ and $20^{\circ} \mathrm{C}$ (Figures 3.2a and S3.1). After this period, different removal trends at the different temperatures were observed. In 54 days, more than $99 \%$ of iopromide was removed at $20^{\circ} \mathrm{C}$ regardless of the initial concentration (Figures 3.2a and S3.1) and the remaining iopromide concentration was below $0.46 \mu \mathrm{M}$. At $5^{\circ} \mathrm{C}$, the removal varied between 50 and $92 \%$ depending on the iopromide initial concentration (Figures 3.2a and S3.1), and a similar removal was observed with both bGAC and autoclaved GAC (Figure 3.2).
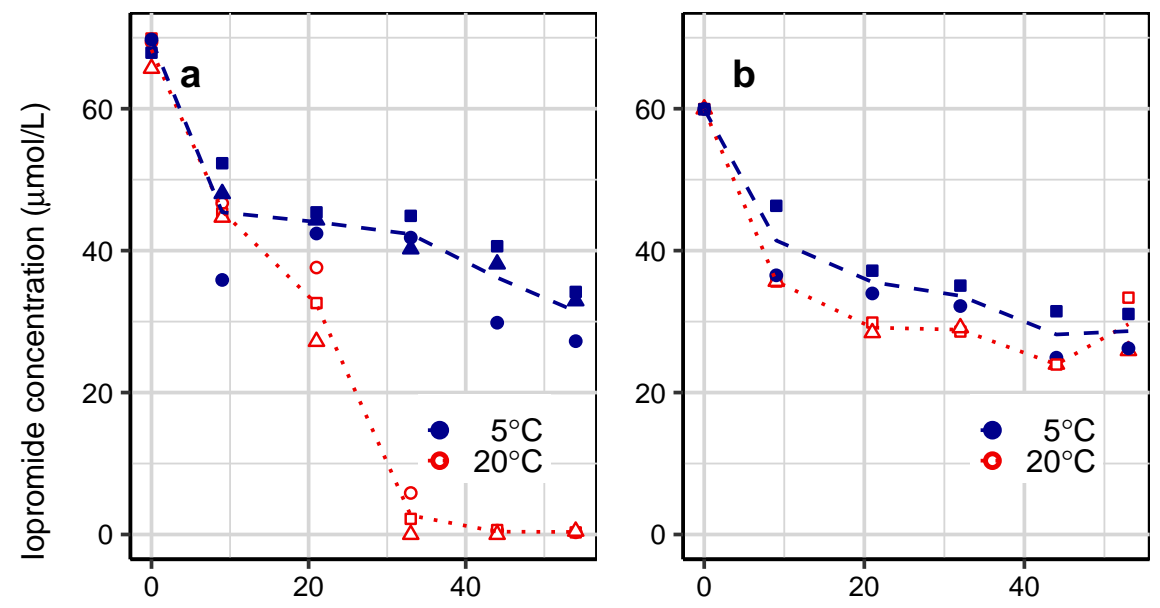

Time (d)

Figure 3.2: Iopromide concentration in time at $5^{\circ} \mathrm{C}$ and $20^{\circ} \mathrm{C}$ with bGAC (a) and autoclaved GAC (b). Different symbols represent different replicates of the same treatment. Lines connect the mean value of replicates in each time point. 
Our results with autoclaved GAC show that iopromide adsorption increased with increasing temperature (Figure 3.2b). It is known that increased temperatures increase diffusion rates, and diffusion can limit adsorption (Taghdiri and Zamani, 2013). However, this is not the reason for the higher iopromide adsorption at $20^{\circ} \mathrm{C}$ given that the adsorption rate at both temperatures was similar. Alternatively, these results might indicate that iopromide adsorption is an endothermic process. Endothermic adsorption of compounds such as ibuprofen and rhodamine B has been reported and attributed to the creation of adsorption sites at higher temperatures (Dubey et al., 2010; Lacerda et al., 2015). The comparison of iopromide removal at both temperatures by bGAC and autoclaved GAC supports the hypothesis that biodegradation is a relevant removal mechanism with bGAC.

Iopromide removal in bGAC filters has been reported in literature. Altmann et al. (2016) observed more than $50 \%$ iopromide removal in a bGAC filter treating secondary effluent from a WWTP. Removal remained stable even after prolonged operation (25,000 bed volumes) and was attributed to iopromide adsorption and biodegradation. Zhang et al. (2017) reported up to $60 \%$ iopromide removal for micropollutants in bGAC filters. The authors hypothesize that biodegradation was the main removal mechanism based on the age of the bGAC (2 years) and on the amount of organic matter (around $2 \mathrm{mg} / \mathrm{L}$ ) present in the influent. In this paper we present additional evidence that iopromide can be degraded by biomass growing in bGAC filters.

Iopromide biodegradation results in recalcitrant transformation products and full mineralization has not yet been reported (Müller et al., 2019). Peaks with the same exact mass (within $5 \mathrm{ppm}$ ) and isotope pattern corresponding to 3 iopromide biodegradation products described by Schulz et al. (2008) were detected in our experiments with bGAC (Figure S3.2). The detection of iopromide degradation products is additional evidence of iopromide biodegradation.

Similarly to iopromide, metformin was biodegraded at $20^{\circ} \mathrm{C}$ with bGAC (Figure $3.3 \mathrm{a}$ and Figure S3.3a, c). Initially, metformin removal was higher in experiments at $5^{\circ} \mathrm{C}$ than $20^{\circ} \mathrm{C}$ (32\% and $20 \%$ average removal on day 9 at $5^{\circ} \mathrm{C}$ and $20^{\circ} \mathrm{C}$ respectively), indicating that metformin adsorption is an exothermic process. This period lasted 9 days for the lowest initial concentration $(25 \mu \mathrm{M})$ and 21 days for the intermediate and highest initial concentrations (respectively $45 \mu \mathrm{M}$ and $69 \mu \mathrm{M}$ ). After that, metformin biodegradation was observed in 6 out of 9 bottles at $20^{\circ} \mathrm{C}$ and its removal was more than $99 \%$ by day 33, regardless of the initial concentration. A further evidence for metformin biodegradation at 
$20^{\circ} \mathrm{C}$ is the production of its first degradation product guanylurea (Figure 3.3a and Figure S3.3a, c). Guanylurea concentration increased in all cases where metformin was biodegraded, and correlated strongly in time with the biodegradation of metformin. The experiments with autoclaved GAC show that both metformin and guanylurea were removed to a larger extent at $5^{\circ} \mathrm{C}$ than at $20^{\circ} \mathrm{C}\left(24 \%\right.$ and $15 \%$ metformin removal at $5^{\circ} \mathrm{C}$ and $20^{\circ} \mathrm{C}$ respectively, $87 \%$ and $79 \%$ guanylurea removal at $5^{\circ} \mathrm{C}$ and $20^{\circ} \mathrm{C}$ respectively), similarly to what was observed in the first days with bGAC (Figure S3.3). This shows that the adsorption of metformin and guanylurea is exothermic.

After guanylurea was produced, it was further removed due to either adsorption or biodegradation. Guanylurea removal between day 33 and 54 cannot be explained by adsorption alone (Figure 3.3a and Figure S3.3a, c). Adsorption happens initially at a fast rate and slows down in time (Piai et al., 2019). However, our results show the opposite trend: guanylurea removal rate is initially lower (between days 33 and 44) and becomes higher between days 44 and 54 . This trend is more consistent with removal due to biodegradation then removal due to adsorption. Initially, guanylurea has been described as the dead-end metformin biodegradation product (Trautwein and Kümmerer, 2011). However, recent studies have reported guanylurea biodegradation by activated sludge or cultures enriched from activated sludge (Briones et al., 2018; Tisler and Zwiener, 2019), indicating that guanylurea can be biodegraded, as also observed in our experiments.
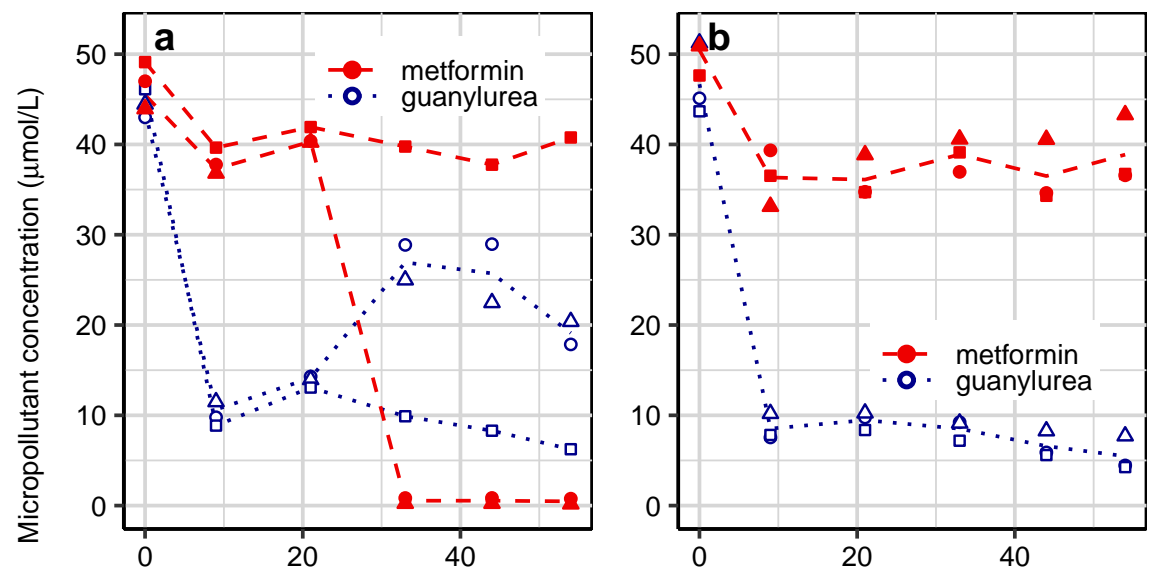

Time (d)

Figure 3.3: Metformin and guanylurea concentration in time at $20^{\circ} \mathrm{C}(\mathrm{a})$ and $5^{\circ} \mathrm{C} \mathrm{(b)} \mathrm{in} \mathrm{experiment} \mathrm{with}$ bGAC. Different symbols represent different replicates of the same treatment. Lines connect the mean value of replicates in each time point. 
Metformin biodegradation and guanylurea production were not observed in all replicates. Metformin biodegradation was observed in the 3 replicates with initial concentration of $25 \mu \mathrm{M}$ (Figure S3.3a), but in only 2 out of 3 replicates with initial concentration of $45 \mu \mathrm{M}$ (Figure 3.3a) and in only 1 out of 3 replicates with initial concentration of $69 \mu \mathrm{M}$ (Figure S3.3c). Therefore, replicates at $20^{\circ} \mathrm{C}$ in which metformin was not degraded were not included in the mean calculation in those figures. We also observed variability between replicates in an independent experiment performed in similar conditions as described in 2.2 and 2.3. In this experiment, metformin was biodegraded in 2 out of 3 replicates (Figure S3.4).

Several authors have reported a similar variability between replicates in metformin biodegradation studies (Markiewicz et al., 2017a, 2017b; Poursat et al., 2019; Trautwein and Kümmerer, 2011). Poursat et al. (2019) reported 99\%, 28\% and 7\% metformin biodegradation for 3 replicates in experiments with sludge pre-exposed for 9 months to $1.5 \mathrm{mg} / \mathrm{L}$ metformin as initial concentration. Markiewicz et al. (2017a) studied metformin biodegradation by activated sludge following the protocol of OECD 301F. In their experiments, replicate bottles removed metformin to different extents ( 0 vs. $30 \%$ and $50 \%$ vs. $100 \%$ in two independent experiments). The authors hypothesize that the large variation in the results was due to the diversity of the microbial community in the inoculum and concluded that metformin biodegradation might occur only if specific degraders are present. Since part of the inoculum used in our experiments consisted of bGAC and collecting a homogeneous sample of this material is difficult, inoculum heterogeneity is likely the reason for the different behaviour of our replicates.

Given the different effects of temperature on micropollutants adsorption, two scenarios can be distinguished in our study. In the first one, adsorption of a micropollutant decreases with increasing temperature (exothermic process). In this case, temperature is negatively correlated to adsorption but positively correlated to biodegradation. Therefore a higher removal at $20^{\circ} \mathrm{C}$ is a strong indication for micropollutant biodegradation, which is the case for metformin. In the second situation, adsorption of a micropollutant increases with increasing temperature (endothermic process). In this case temperature is positively correlated to both adsorption and biodegradation and a higher removal at $20^{\circ} \mathrm{C}$ does not necessarily imply that the compound is biodegraded. Therefore, the removal trends at both temperatures, combined with the removal with autoclaved GAC, can indicate whether the compound was biodegraded or not. This is what we have shown for iopromide removal. 
In our experiments, a positive correlation between temperature and adsorption was also observed for iopamidol (Figure 3.4). Iopamidol removal at $20^{\circ} \mathrm{C}$ with bGAC was higher than with autoclaved GAC, whereas at $5^{\circ} \mathrm{C}$ iopamidol was removed to a similar extent in both experiments (Figure 3.4). At the end of the experiments at $20^{\circ} \mathrm{C}, 45 \%$ of iopamidol was removed with autoclaved GAC, whereas $68 \%$ was removed with bGAC. This indicates that iopamidol was biodegraded in the later. A peak with exact mass (within $5 \mathrm{ppm}$ ) and isotope pattern corresponding to an iopamidol biodegradation product described by Kormos et al. (2010) was detected in experiments with bGAC cultivated at $20^{\circ} \mathrm{C}$ (Figure S3.5), but not when cultivated at $5^{\circ} \mathrm{C}$. This supports the hypothesis of iopamidol biodegradation. An alternative explanation for the higher iopamidol removal with bGAC at $20^{\circ} \mathrm{C}$ is related to iopromide and metformin biodegradation. Biodegradation of the dissolved fraction of iopromide and metformin creates a driving force for the desorption of these compounds from the bGAC. This releases adsorption sites on the GAC surface, which might have contributed to increasing iopamidol removal due to adsorption.
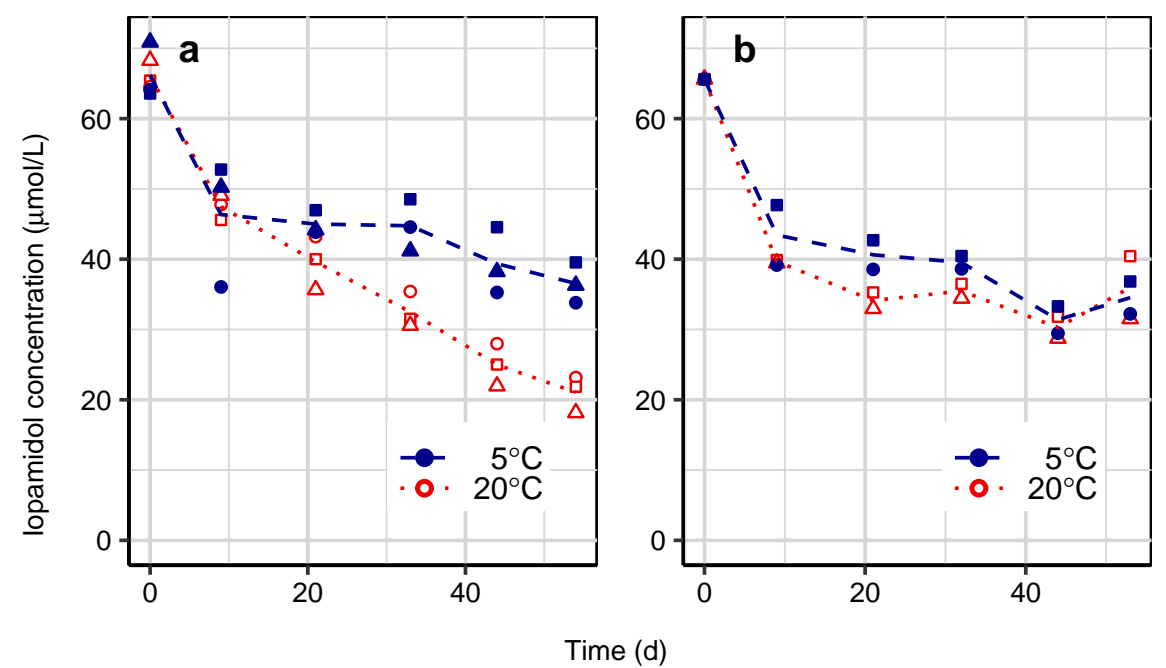

Figure 3.4: Iopamidol concentration in time at $5^{\circ} \mathrm{C}$ and $20^{\circ} \mathrm{C}$ with bGAC (a) and autoclaved GAC (b). Different symbols represent different replicates of the same treatment. Lines connect the mean value of replicates in each time point.

Different from iopromide and iopamidol, diclofenac removal was higher with bGAC than with autoclaved GAC at both temperatures (Figure 3.5). As a result, it is difficult to assess whether diclofenac was biodegraded or only adsorbed onto bGAC. A possible explanation is that diclofenac was biodegraded in bottles cultivated at both $5^{\circ} \mathrm{C}$ and $20^{\circ} \mathrm{C}$ with bGAC. However, we expect that the biodegradation rate at $5^{\circ} \mathrm{C}$ would not be significant enough to 
explain the removal observed. Furthermore, peaks corresponding to diclofenac transformation products described by Kosjek et al. (2009) were not found in any of the samples from day 54 in either temperature. Therefore, we could not elucidate if biodegradation was an additional removal mechanism for diclofenac with bGAC.
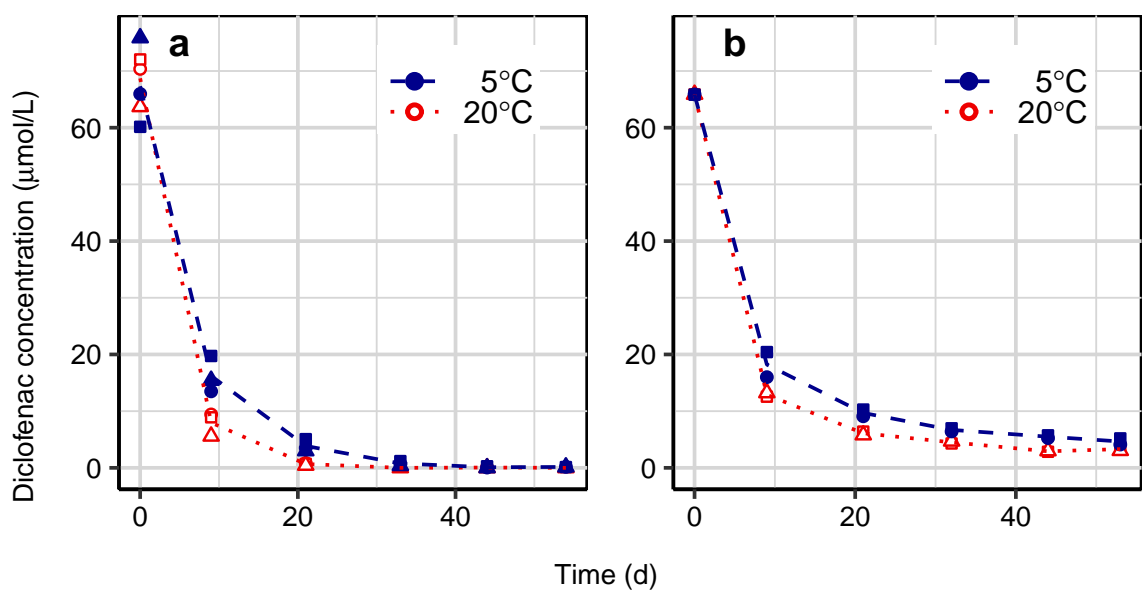

Figure 3.5: Diclofenac concentration in time at $5^{\circ} \mathrm{C}$ and $20^{\circ} \mathrm{C}$ with bGAC (a) and autoclaved GAC (b). Different symbols represent different replicates of the same treatment. Lines connect the mean value of replicates in each time point.

\subsubsection{Temperature effect on adsorption of non-biodegraded micropollutants}

We observed no trends indicating biodegradation of the following micropollutants with bGAC: benzotriazole, desphenyl-chloridazon, hexamethylenetetramine, melamine and pyrazole. For these compounds, we found that adsorption was the only removal mechanism, both with bGAC as well as with autoclaved GAC. Therefore, results from both experiments were used to evaluate the effect of temperature on adsorption of these micropollutants in different concentrations.

Melamine and desphenyl-chloridazon were removed to a larger extent at $5{ }^{\circ} \mathrm{C}$ than at $20^{\circ} \mathrm{C}$, both with bGAC and autoclaved GAC (Figure 3.6 and Figure S3.6). This indicates that adsorption of melamine and desphenyl-chloridazon is exothermic, similarly to metformin and guanylurea. Interestingly, the difference between melamine and desphenyl-chloridazon removal at $5^{\circ} \mathrm{C}$ and $20^{\circ} \mathrm{C}$ reduced with increasing micropollutant initial concentration. This can be explained based on the increased coverage of the GAC surface at increasing micropollutant initial concentrations. Studies show that at higher GAC coverage, the temperature dependence of adsorption is reduced (Hindarso et al., 2001; Pikaar et al., 2006; 
Sander and Pignatello, 2005), which is related to the presence of sites with different adsorption affinities on the AC surface. High affinity adsorption sites are the first to be occupied by the adsorbate and the temperature dependence of adsorption is higher in those sites. Low affinity adsorption sites are occupied only at higher AC surface coverage and the temperature dependence of these sites is smaller or negligible (Pikaar et al., 2006).
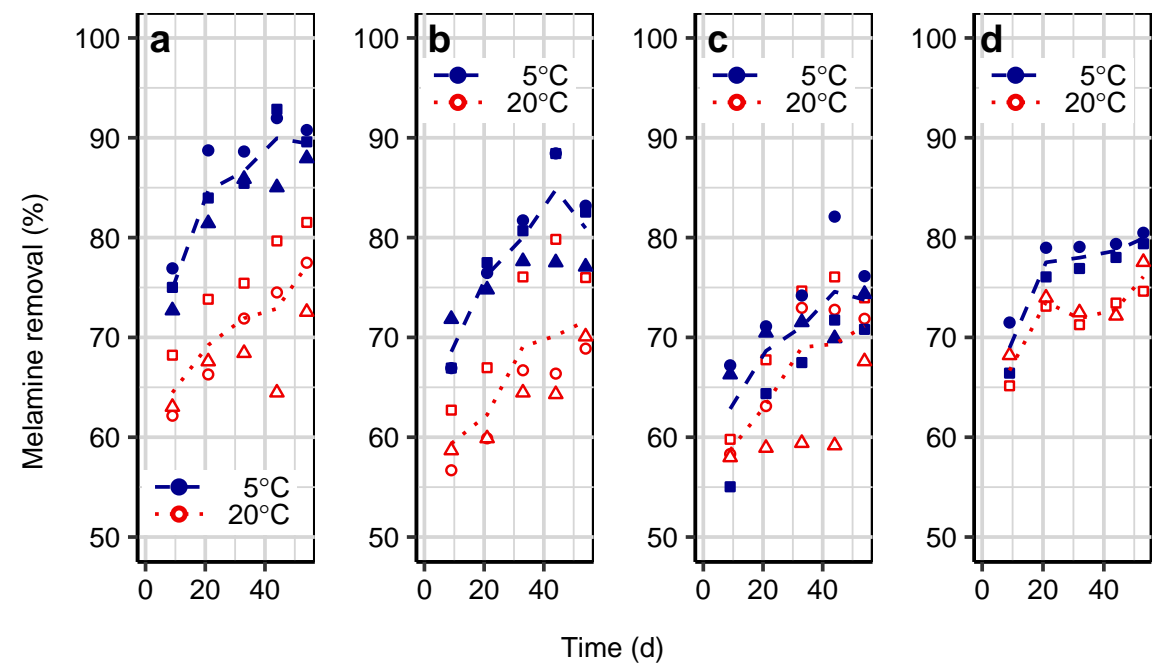

Time (d)

Figure 3.6: Melamine removal with bGAC at 3 different initial concentrations - a) $25 \mu \mathrm{M}$, b) $45 \mu \mathrm{M}$, c) 69 $\mu \mathrm{M}$ - and autoclaved GAC - d) $69 \mu \mathrm{M}$ - at $5^{\circ} \mathrm{C}$ and $20^{\circ} \mathrm{C}$. Different symbols represent different replicates of the same treatment. Lines connect the mean value of replicates in each time point. Y-axis scale runs from $50 \%-100 \%$ for better visualization of the data.

At an initial concentration of $69 \mu \mathrm{M}$, desphenyl-chloridazon was removed to a larger extent at $5^{\circ} \mathrm{C}$ than at $20^{\circ} \mathrm{C}$ with autoclaved GAC (Figure S3.5d), but it was removed to the same extent at both temperatures with bGAC (Figure S3.5c). This difference can be related to a different AC coverage in each experiment. As previously mentioned, the temperature dependence of adsorption can reduce at higher AC coverage. Autoclaving the AC might have affected the biofilm and/or organic matter previously adsorbed to the $\mathrm{AC}$, reducing its coverage.

Hexamethylenetetramine was removed to a larger extent at $20^{\circ} \mathrm{C}$ both with bGAC and autoclaved GAC (Figure 3.7). Different from our results, a negative correlation between hexamethylenetetramine adsorption and temperature was found by Taghdiri and Zamani (2013). Differences between their work and ours include use of column experiments instead of batch, a different type of AC, powder AC instead of GAC and a shorter contact time (200 
min instead of 54 days). However, we could not explain the difference between our results and theirs based on the difference in experimental setup.
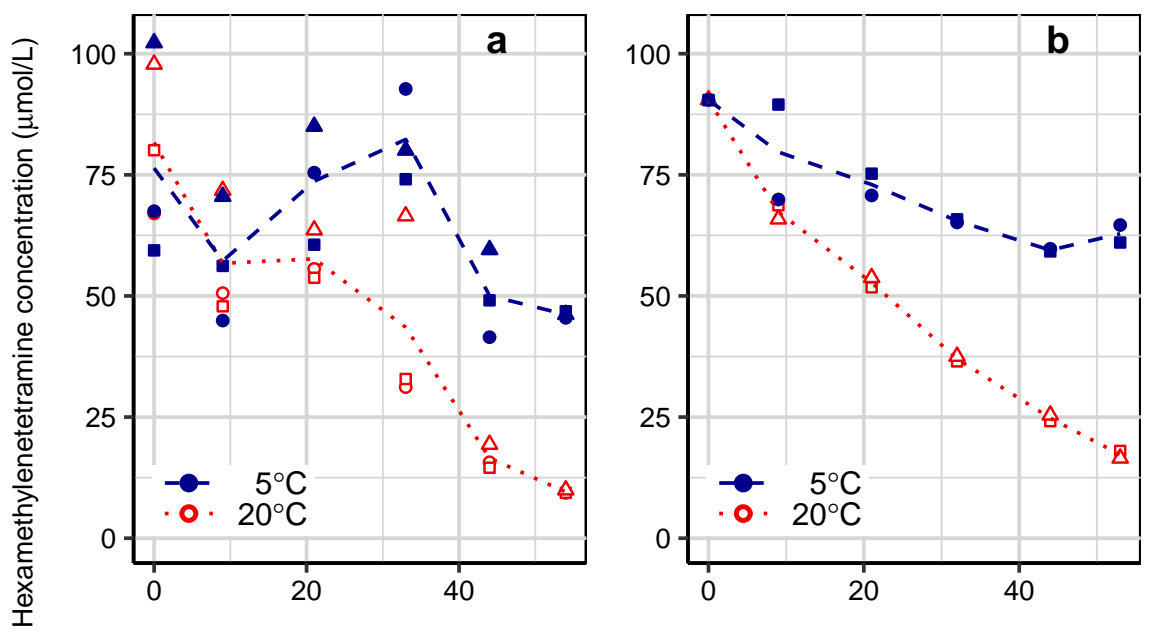

Time (d)

Figure 3.7: Hexamethylenetetramine concentration in time at $5{ }^{\circ} \mathrm{C}$ and $20^{\circ} \mathrm{C}$ with bGAC (a) and autoclaved GAC (b). Different symbols represent different replicates of the same treatment. Lines connect the mean value of replicates in each time point.

No difference in pyrazole and benzotriazole removal between temperatures was observed, neither with bGAC, nor with autoclaved GAC (data not shown). This indicates that these compounds were not biodegraded and that adsorption is not strongly affected by temperature. To the best of our knowledge, the effect of temperature on the adsorption of these compounds onto GAC has not been reported in literature.

Benzotriazole, iopamidol and melamine were not biodegraded in our experiments with bGAC, even though their biodegradation has been reported in literature (El-Sayed et al., 2006; Herzog et al., 2014; Kormos et al., 2010; Wagner et al., 2020). These studies applied constructed wetlands or used activated sludge, WWTP effluent or soil impacted by WWTP effluent as inoculum. We hypothesize that the biodegradation potential for micropollutants is smaller in GAC filters used in drinking water production than in those systems, for various reasons. One possible reason is the fact that micropollutants concentrations are expected to be higher in constructed wetlands and WWTP than in DWTP. Another reason is the higher concentration of dissolved organic carbon in wastewater, compared to drinking water (Zietzschmann et al., 2016b), which creates favourable conditions for biodegradation of micropollutants via cometabolic routes (Tran et al., 2013). Still, GAC filters harbour a 
diverse microbial community with high metabolic versatility (Oh et al., 2018). We have demonstrated that biofilm growing on used GAC from a DWTP can biodegrade at least 3 out of the 10 micropollutants tested. Moreover, we propose that experiments involving micropollutants removal at different temperatures can be used to assess their biodegradability by bGAC.

\subsubsection{Adsorption capacity of used and fresh GAC}

We compared the adsorption capacity of used and fresh GAC to gain insight in the possible loss of adsorption capacity due to GAC use. For this purpose, we compared the micropollutant removal of fresh GAC (see 2.4) with MP removal by bGAC (initial concentration $69 \mu \mathrm{M}$ ) on day 9. Despite the hydrophilic character of these micropollutants, as indicated by their $\log D_{\text {OW }}$ (Table 3.1), more than $90 \%$ removal was achieved for 5 out of 10 compounds (Table 3.2). A more detailed discussion regarding affinity of these micropollutants towards GAC, including adsorption isotherms, is presented in Piai et al. (2019).

The loss of adsorption capacity due to GAC use differs considerably per compound (Table 3.2). The adsorption capacity of benzotriazole and diclofenac by used GAC is respectively only $16 \%$ and $23 \%$ lower than of fresh GAC. This shows that even though GAC was used for more than 100,000 bed volumes, the adsorption capacity for these micropollutants was still high. Guanylurea, metformin and hexamethylenetetramine adsorbed to a larger extent onto used GAC than onto fresh GAC. The higher removal of guanylurea and metformin with used GAC can be related to the increased electrostatic attraction between the negatively charged biofilm on the used GAC surface and these positively charged micropollutants (Briones and Sarmah, 2018). Increased removal of cationic compounds due to biofilm growth on GAC surface has already been reported (Yin et al., 2007). The higher removal of hexamethylenetetramine with used GAC compared to fresh GAC cannot be explained based on charge interactions, given that this compound is neutral at the $\mathrm{pH}$ of the experiment. This result might be related to the unexpectedly high initial concentration of hexamethylenetetramine in one of the replicates in the experiment with bGAC (Figure 3.7a). 
Table 3.2: Micropollutant removal by used and fresh GAC at $5^{\circ} \mathrm{C}$ and relative removal of used and fresh GAC. Values correspond to mean of triplicates for used GAC and duplicates for fresh GAC.

\begin{tabular}{|c|c|c|c|}
\hline \multirow[t]{2}{*}{ Micropollutants } & \multicolumn{3}{|c|}{ Removal (\%) } \\
\hline & Fresh GAC & Used GAC & Used/fresh GAC \\
\hline Benzotriazole & 99 & 83 & 84 \\
\hline Desphenyl-chloridazon & 99 & 66 & 67 \\
\hline Diclofenac & 97 & 75 & 77 \\
\hline Guanylurea & 52 & 77 & 148 \\
\hline Hexamethylenetetramine & 14 & 23 & 161 \\
\hline Iopamidol & 90 & 30 & 33 \\
\hline Iopromide & 93 & 35 & 38 \\
\hline Melamine & 94 & 63 & 67 \\
\hline Metformin & 12 & 30 & 250 \\
\hline Pyrazole & 51 & 28 & 55 \\
\hline
\end{tabular}

The loss of adsorption capacity due to GAC use was the highest for iopamidol, iopromide and pyrazole. Iopamidol and iopromide are the largest molecules in the experiment, which means that they are most affected by pre-adsorbed organic matter onto used GAC, due to pore blockage and competition for adsorption sites (Zietzschmann et al., 2015). The difference between adsorption capacity of used and fresh GAC for the different micropollutants can also be partially explained by the previous exposure of the used GAC to some micropollutants. It is expected that less adsorption sites will remain available for the compounds to which the GAC has been more exposed (Greenstein et al., 2018). However, with the available data, it is not possible to conclude if that is the reason why the loss of adsorption capacity was high for pyrazole.

\subsection{Conclusions}

In this study, we have shown that biofilm growing in GAC filters at a DWTP can biodegrade iopromide, metformin and iopamidol. In addition, we have shown that temperature is positively correlated to adsorption of iopromide, iopamidol, diclofenac and hexamethylenetetramine, but negatively correlated to adsorption of desphenyl-chloridazon, guanylurea, melamine and metformin. We could also demonstrate that the adsorption capacity of GAC used for more than 100,000 bed volumes is comparable to adsorption of fresh GAC for diclofenac and benzotriazole and higher for guanylurea, metformin and hexamethylenetetramine. 
Our experimental set-up of comparing micropollutants removal at different temperatures can be used to distinguish biodegradation from adsorption in used GAC. The distinction between these two processes allows to assess the potential of biofilm growing in GAC filters to biodegrade micropollutants and to elucidate the contribution of biodegradation as a removal process for micropollutants in these filters during drinking water production. This study contributes to more insights related to bioregeneration of loaded GAC. Demonstrating that sorption and biodegradation occur simultaneously and sufficiently, and leads to an efficient bioregeneration of the GAC, can aid in reducing the thermal regeneration that is currently used for GAC filters at DWTP.

\section{Acknowledgements}

This work was financially supported by Evides Water Company N.V. (Rotterdam, The Netherlands). We also want to thank Andrea Brunsch for the inspiration for the experimental design and Baptiste Poursat for sharing insights and discussion about intravariability of experimental replicates. Finally, we would like to thank Andrea AldasVargas, Koen van Gijn and Thomas Wagner for proof reading the manuscript. 


\section{Supplementary information to Chapter 3}

Table S3.1: Composition of concentrated nutrients solution used to prepare media for micropollutants removal experiments with bGAC and autoclaved GAC.

\section{Concentration $(\mathrm{g} / \mathrm{L})$}

\begin{tabular}{|c|c|}
\hline Buffer & \\
\hline $\mathrm{Na}_{2} \mathrm{HPO}_{4} .2 \mathrm{H}_{2} \mathrm{O}$ & 35,6 \\
\hline $\mathrm{KH}_{2} \mathrm{PO}_{4}$ & 27,2 \\
\hline \multicolumn{2}{|l|}{ Macro nutrients } \\
\hline $\mathrm{NH}_{4} \mathrm{Cl}$ & 170 \\
\hline $\mathrm{CaCl}_{2} \cdot 2 \mathrm{H}_{2} \mathrm{O}$ & 8 \\
\hline $\mathrm{MgSO}_{4} .7 \mathrm{H}_{2} \mathrm{O}$ & 9 \\
\hline \multicolumn{2}{|l|}{ Trace elements } \\
\hline $\mathrm{FeCl}_{2} .4 \mathrm{H}_{2} \mathrm{O}$ & 2 \\
\hline $\mathrm{CoCl}_{2} \cdot 6 \mathrm{H}_{2} \mathrm{O}$ & 2 \\
\hline $\mathrm{MnCl}_{2} .4 \mathrm{H}_{2} \mathrm{O}$ & 0.5 \\
\hline $\mathrm{CuCl}_{2} .2 \mathrm{H}_{2} \mathrm{O}$ & 0.03 \\
\hline $\mathrm{ZnCl}_{2}$ & 0.05 \\
\hline $\mathrm{HBO}_{3}$ & 0.05 \\
\hline$\left(\mathrm{NH}_{4}\right)_{6} \mathrm{Mo}_{7} \mathrm{O}_{24} .4 \mathrm{H}_{2} \mathrm{O}$ & 0.09 \\
\hline $\mathrm{Na}_{2} \mathrm{SeO}_{3} .5 \mathrm{H}_{2} \mathrm{O}$ & 0.1 \\
\hline $\mathrm{NiCl}_{2} .6 \mathrm{H}_{2} \mathrm{O}$ & 0.05 \\
\hline EDTA (tripex 2) & 1 \\
\hline $\mathrm{HCl} 36 \%$ & $1 \mathrm{~mL} / \mathrm{L}$ \\
\hline \multirow[t]{2}{*}{ Na-Resazurin } & 0.5 \\
\hline & Amount used for $1 \mathrm{~L}$ media $(\mathrm{mL})$ \\
\hline Buffer & $\begin{array}{ll} & 50\end{array}$ \\
\hline Macro nutrients & 6 \\
\hline Trace elements & 0.6 \\
\hline
\end{tabular}


Table S3.2: Quality parameters of GAC filter influent used to prepare media for micropollutants removal experiments with bGAC and autoclaved GAC.

\begin{tabular}{lr}
\hline Parameter & Value \\
\hline Total Organic Carbon $(\mathrm{mg}$ C/L) & 2.7 \\
Dissolved Organic Carbon $(\mathrm{mg} \mathrm{C} / \mathrm{L})$ & 2.1 \\
UV-extinction at $254 \mathrm{~nm}(1 / \mathrm{m})$ & 5.6 \\
$\mathrm{pH}$ & 7.9 \\
Suspended solids $(\mathrm{mg} / \mathrm{L})$ & $<0.1$ \\
\hline
\end{tabular}

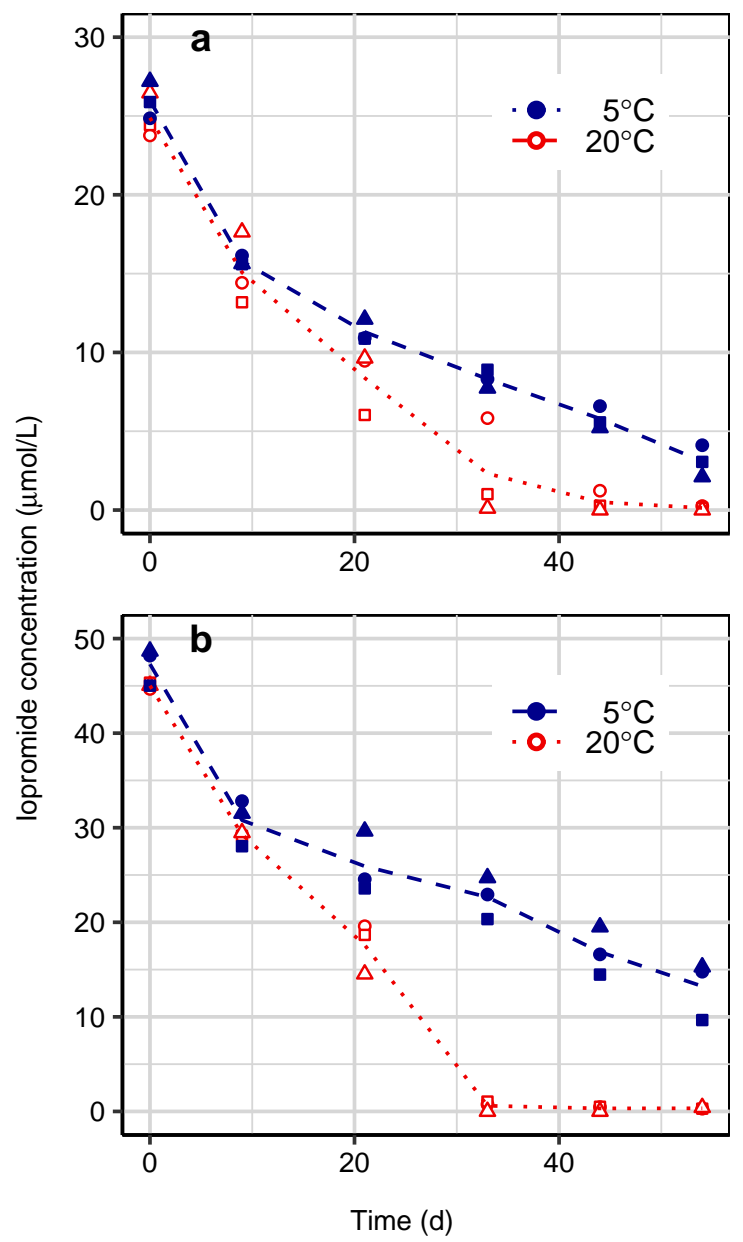

Figure S3.1: Iopromide concentration in time with bGAC at $5^{\circ} \mathrm{C}$ and $20^{\circ} \mathrm{C}$ and 2 different initial concentrations: a) $25 \mu \mathrm{M}$ and b) $45 \mu \mathrm{M}$. Different symbols represent different replicates of the same treatment. Lines connect the mean value of replicates in each time point. 

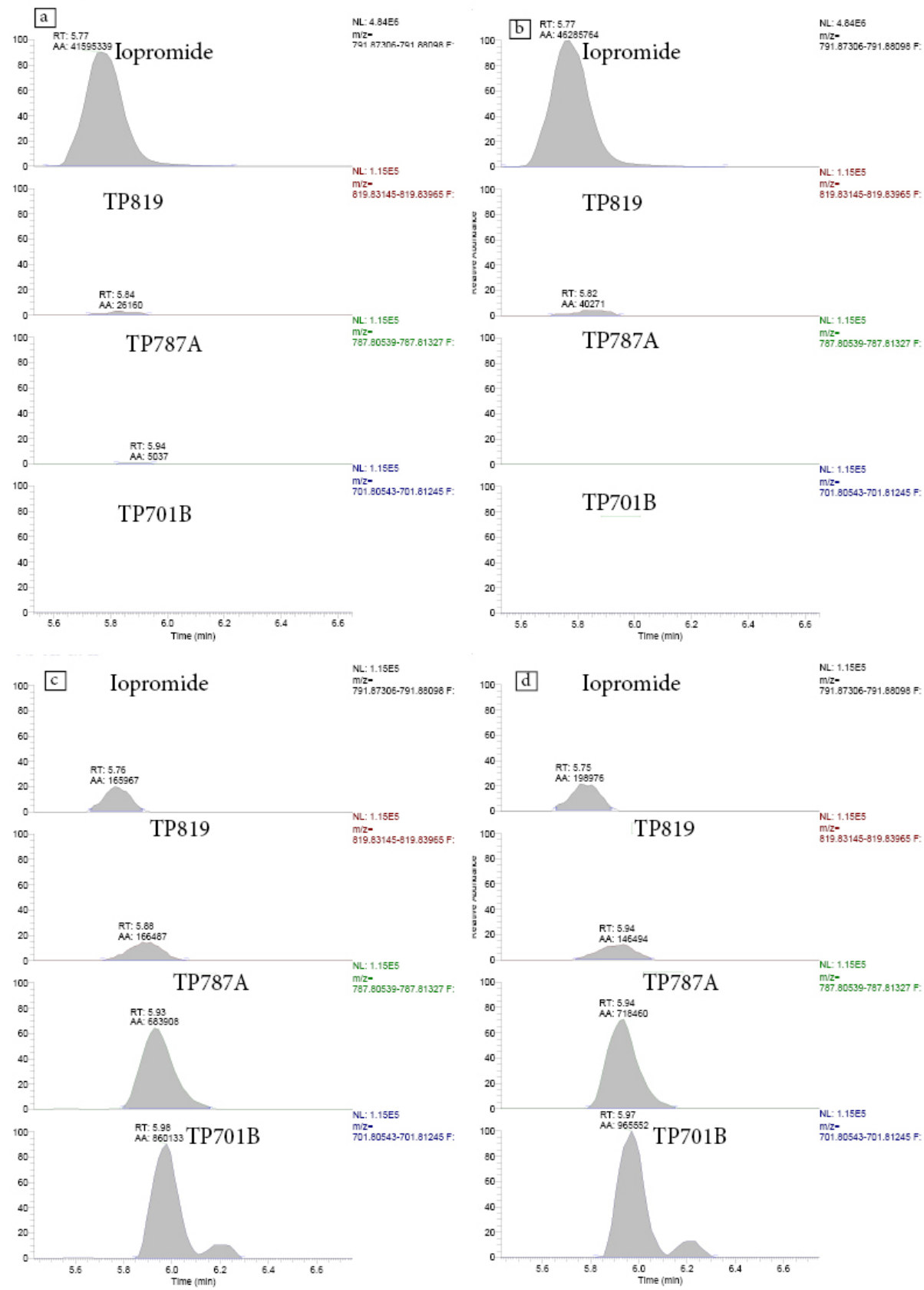

Figure S3.2: Peaks corresponding to $\mathrm{m} / \mathrm{z}$ of iopromide and iopromide degradation products described by Schulz et al. (2008) on day 54 . a and b: replicates cultivated at $5^{\circ} \mathrm{C}$. c and d: replicates cultivated at $20^{\circ} \mathrm{C}$. 

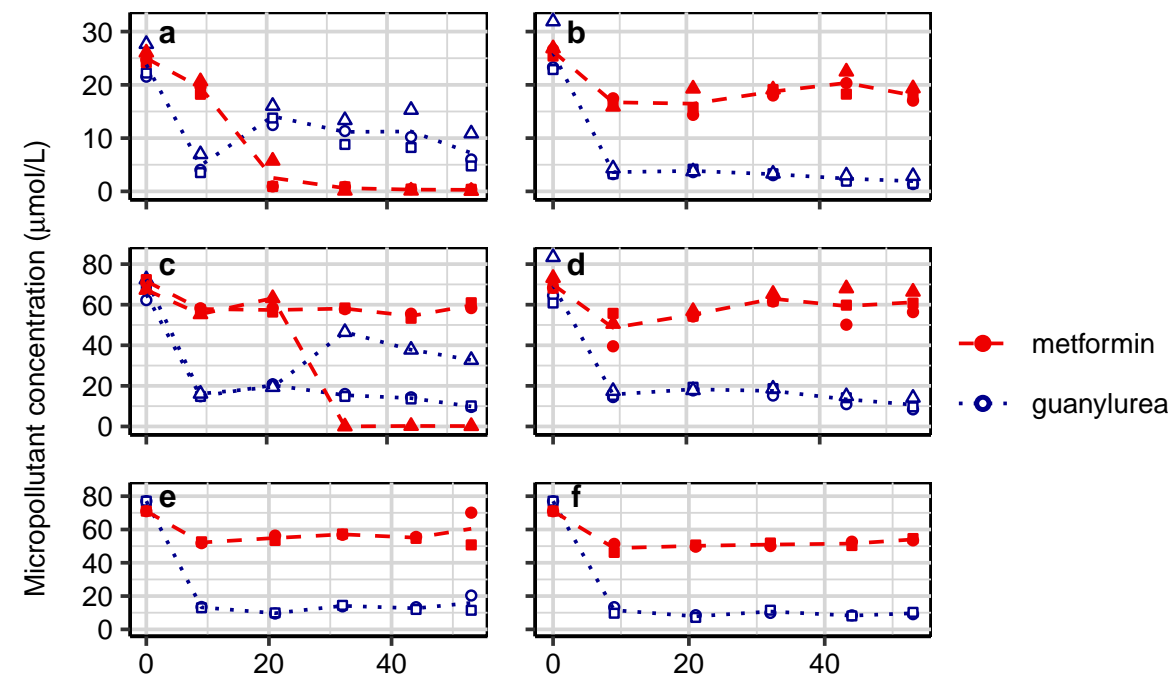

Time (d)

Figure S3.3: Metformin and guanylurea concentration in time at different initial concentrations and different temperatures: a) bGAC, $20^{\circ} \mathrm{C}, 25 \mu \mathrm{M}$; b) bGAC, $5^{\circ} \mathrm{C}, 25 \mu \mathrm{M}$; c) bGAC, $20^{\circ} \mathrm{C}, 69 \mu \mathrm{M}$; d) bGAC, $5^{\circ} \mathrm{C}, 69 \mu \mathrm{M}$, e) autoclaved GAC $20^{\circ} \mathrm{C}, 69 \mu \mathrm{M}$;f) autoclaved GAC, $5^{\circ} \mathrm{C}, 69 \mu \mathrm{M}$. Different symbols represent different replicates of the same treatment. Lines connect the mean value of replicates in each time point.
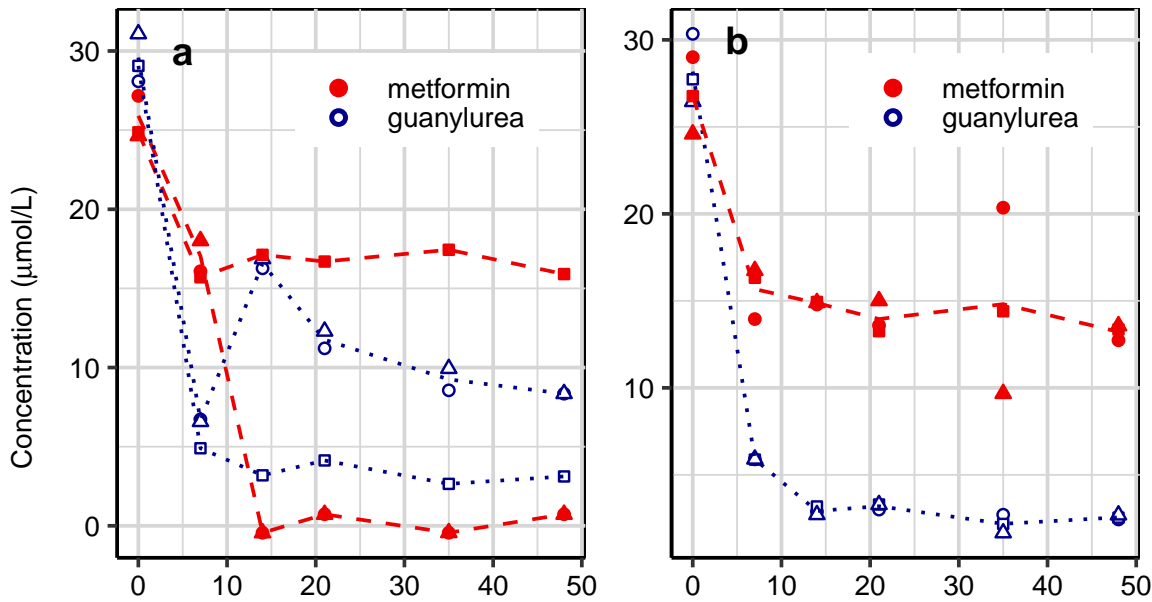

Time $(d)$

Figure S3.4: Metformin and guanylurea concentration in time with bGAC (a) and autoclaved GAC (b). Results were obtained in experiment performed independently from the one presented in Figure 3.3 and Figure S3.3. Different symbols represent different replicates of the same treatment. Lines connect the mean value of replicates in each time point. Metformin biodegradation (resulting in guanylurea production) was observed in two out of three replicates, similarly to main experiment. 

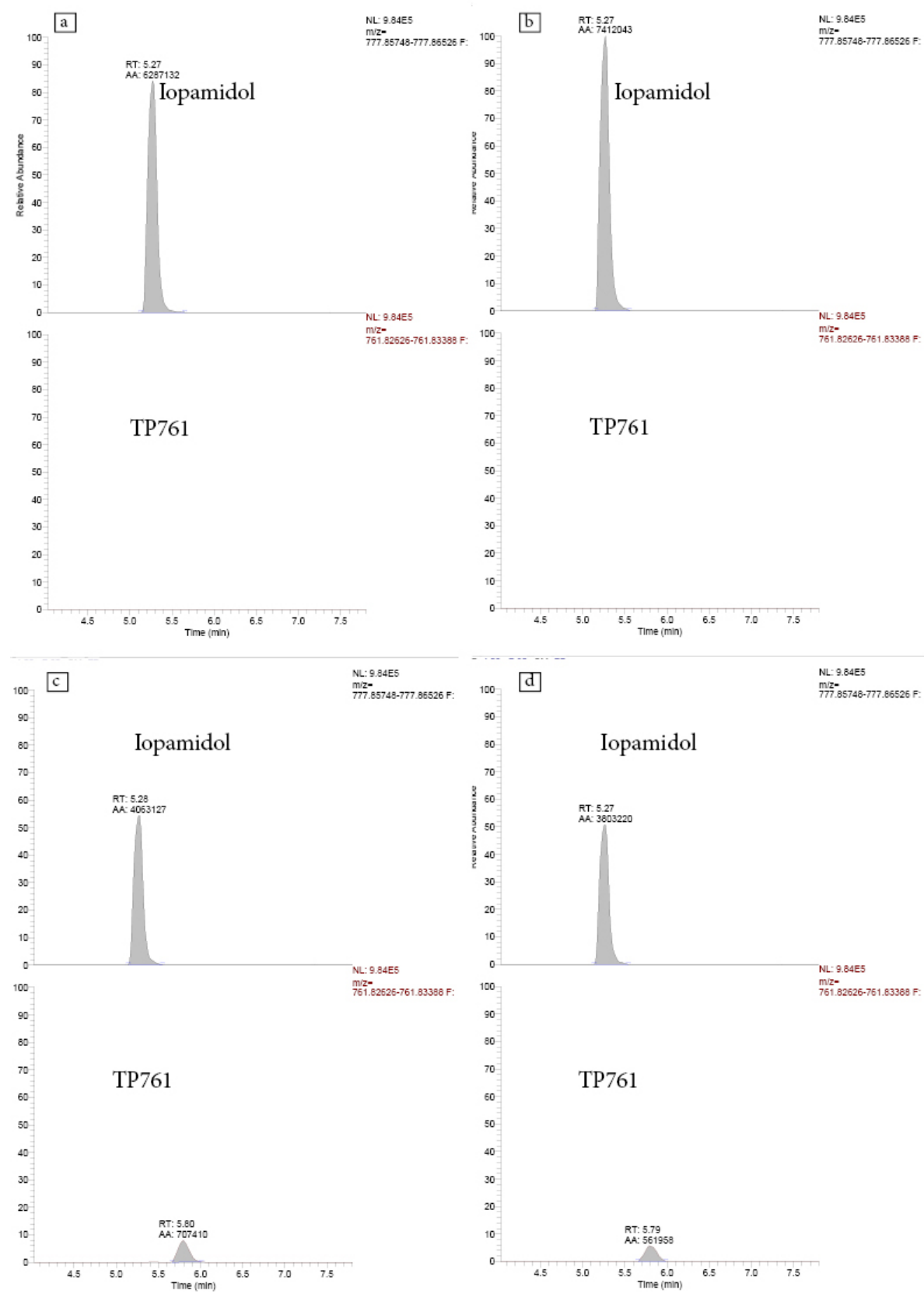

Figure S3.5: Peaks corresponding to $\mathrm{m} / \mathrm{z}$ of iopamidol and iopamidol degradation products (TP761, described by Kormos et al. (2010)) on day 54. a and b: replicates cultivated at $5^{\circ} \mathrm{C}$. c and d: replicates cultivated at $20^{\circ} \mathrm{C}$. 

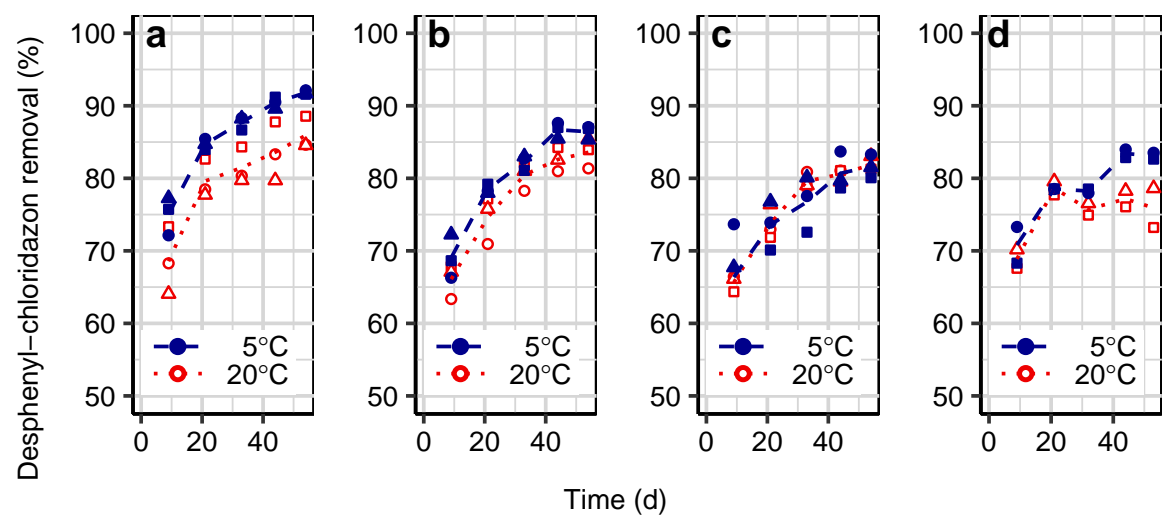

Time (d)

Figure S3.6: Desphenyl-chloridazon removal with bGAC at 3 different initial concentrations - a) $25 \mu \mathrm{M}$, b) $45 \mu \mathrm{M}$, c) $69 \mu \mathrm{M}$ - and autoclaved GAC - d) $69 \mu \mathrm{M}-$ at $5^{\circ} \mathrm{C}$ and $20^{\circ} \mathrm{C}$. Different symbols represent different replicates of the same treatment. Lines connect the mean value of replicates in each time point. Y-axis scale runs from $50 \%-100 \%$ for better visualization of the data. 


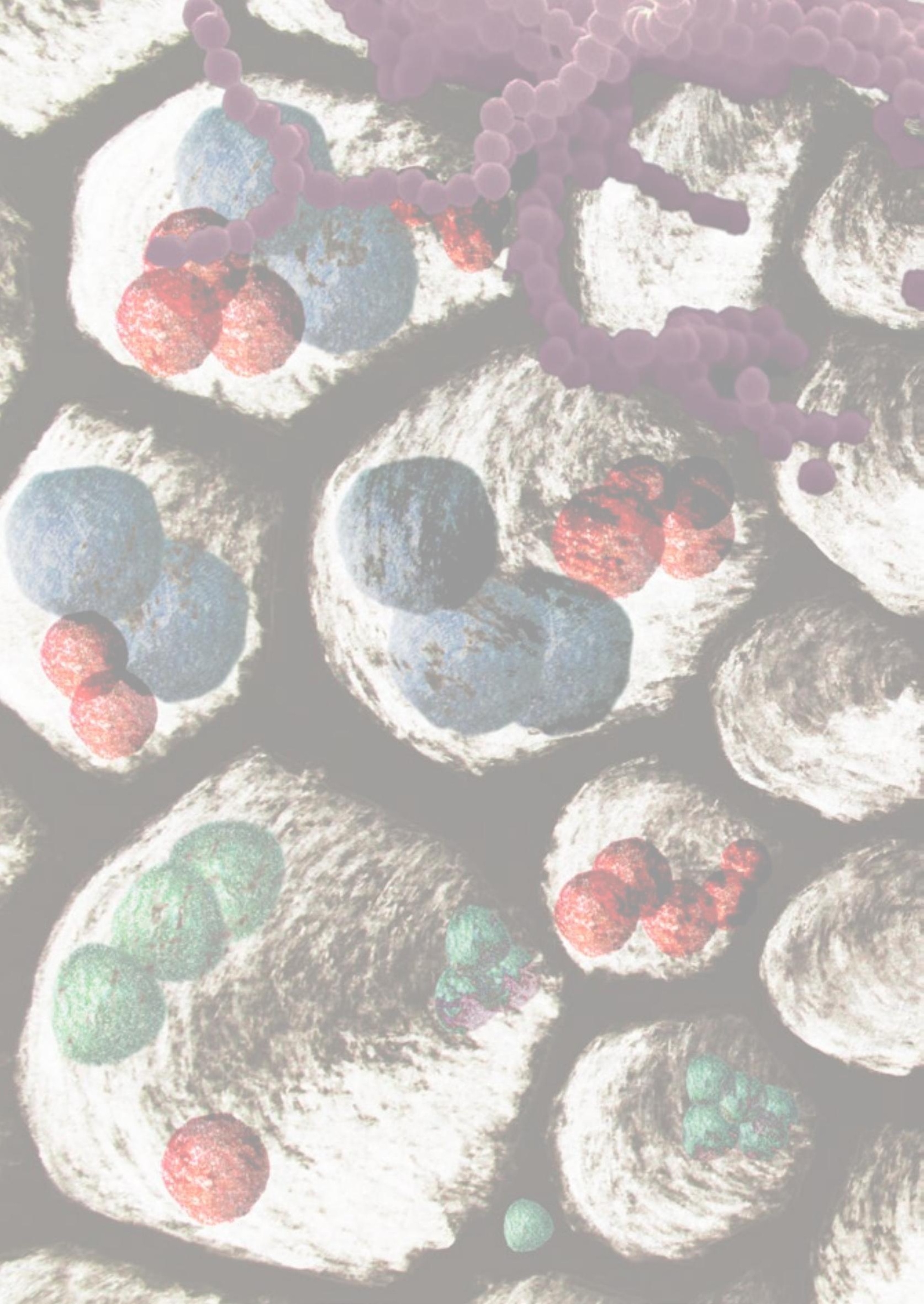




\begin{abstract}
The industrial chemical melamine is often detected in surface water used for drinking water production, due to its wide application and insufficient removal in conventional wastewater treatment plants. Melamine can be removed from water by adsorption onto granular activated carbon (GAC), nevertheless, GAC needs periodic reactivation in costly and energy intense processes. As an alternative method, GAC can also be regenerated using biomass capable of degrading melamine in a process called bioregeneration. We assessed melamine biodegradation in batch experiments in fully oxic and anoxic, as well as in alternating oxic and anoxic conditions. Additionally, we studied the effect of an additional carbon source on the biodegradation. The most favourable conditions for melamine biodegradation were applied to bioregenerate GAC loaded with melamine. We demonstrate that melamine can be biodegraded in either oxic or anoxic conditions and that melamine degrading biomass can restore at least $28 \%$ of the original GAC adsorption capacity. Furthermore, our results indicate that bioregeneration occurs mainly in the largest pore fraction of GAC, impacting adsorption kinetics. Overall, we show that bioregeneration has a large potential for restoring GAC adsorption capacity in industrial wastewater.
\end{abstract}

This chapter has been submitted as:

Piai, L., van der Wal, A., Boelee, N., Langenhoff, A., 2020. Melamine degradation to bioregenerate granular activated carbon. Journal of Hazardous Materials. 


\subsection{Introduction}

Melamine is an organic micropollutant frequently found in the environment (Ruff et al., 2015; Seitz and Winzenbacher, 2017; Zhu et al., 2019). This micropollutant is used as an industrial chemical compound for a wide range of applications, including the synthesis of melamine-formaldehyde resins to produce laminates, plastics and coatings (Smit, 2018). Melamine can be released to the environment both during the production process, as well as by using products containing melamine and its derivatives (Smit, 2018). This micropollutant is poorly removed from water in conventional wastewater treatment plants due to its low biodegradability by and low adsorbability to conventional activated sludge (An et al., 2017; $\mathrm{Xu}$ et al., 2013). As a consequence of insufficient removal in wastewater treatment plants (WWTPs) melamine is discharged into surface water. From 2015 to 2018, melamine was frequently detected in rivers used for the production of drinking at concentrations above the signalling value of $1 \mu \mathrm{g} / \mathrm{L}$ (RIWA-Maas, 2017, 2016; RIWA-Rijn, 2018, 2016). Given its ubiquitous presence in surface water, a temporary derogation limit was proposed by Dutch authorities, allowing the intake of water to produce drinking water with a maximum concentration of $5 \mu \mathrm{g} / \mathrm{L}$ melamine. The possible contamination of drinking water sources with a micropollutant such as melamine highlights the need for improving current water treatment technologies in order to increase the removal of melamine from wastewater and prevent its presence in drinking water.

Micropollutants, such as melamine, can be removed from (waste)water using adsorption to activated carbon (AC) (Guillossou et al., 2019; Stackelberg et al., 2007). AC is a commonly used adsorbent with affinity for several micropollutants, including melamine (Piai et al., 2019). In adsorption processes with AC, the adsorbed compounds accumulate on the AC surface and the AC adsorption capacity decreases with its usage until the AC needs to be either replaced or reactivated (Worch, 2012). Reactivation of AC used in water treatment is most commonly achieved by applying high temperatures $\left(700^{\circ} \mathrm{C}\right.$ to $\left.900^{\circ} \mathrm{C}\right)$ which makes it an energy intensive process (Worch, 2012). Biological regeneration is also possible as long as adsorbates can be desorbed and microorganisms capable of degrading them are present and active (Abromaitis et al., 2016; Aktaş and Çeçen, 2006a).

Microorganisms capable of degrading melamine were first reported by Cook and Huetter (1981). The authors isolated microorganisms from soil, and these microorganisms were capable of degrading melamine by using it as a sole nitrogen source and by using lactate or glucose as carbon source. After that, more authors reported melamine biodegradation using 
other carbon sources, such as glycerol, methanol and ethanol (Shelton et al., 1997; Takagi et al., 2012). Furthermore, Wang et al. (2014a) observed melamine biodegradation without additional carbon sources. Given the low C:N ratio in melamine, an additional carbon source likely increases melamine biodegradation rate by promoting biomass growth.

The biodegradation pathway of melamine was first proposed by Jutzi et al. (1982) and later confirmed by other authors (El-Sayed et al., 2006; Shelton et al., 1997). The biodegradation occurs through consecutive hydrolytic deamination of the ammonia groups attached to the aromatic ring, producing ammeline, ammelide and cyanuric acid, followed by ring cleavage (Figure 4.1). Melamine biodegradation results in release of ammonium, which can accumulate in the media (Takagi et al., 2012). However, in the presence of oxygen and nitrifying microorganisms, ammonium can be oxidized to nitrate. The produced nitrate can then be denitrified if denitrifying microorganisms are present and active. The resulting nitrogen removal through nitrification and denitrification creates nitrogen limiting conditions which can promote further melamine degradation, given that this micropollutant is consumed as a nitrogen source.

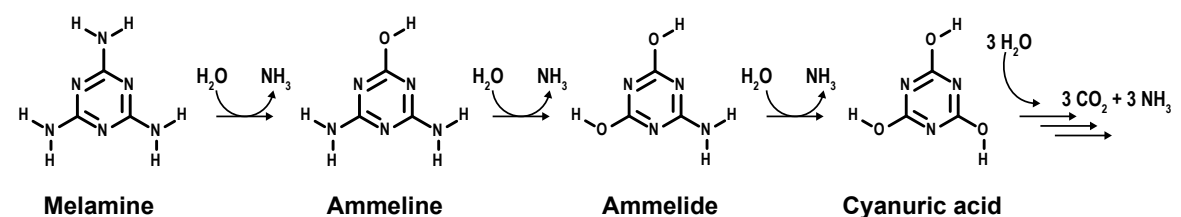

Figure 4.1: Melamine biodegradation pathway (Jutzi et al., 1982).

The combination of biological treatment with granular activated carbon (GAC) offers several advantages compared to biological treatment alone. Higher removal efficiencies of micropollutants with attached growth compared to suspended growth processes have been reported, as a consequence of washout of slow growing organisms in suspended systems (Falås et al., 2013). Furthermore, GAC is known to be an excellent carrier material for biomass since adsorption can remove toxic or inhibitory compounds that might be present in the water (Çeçen and Aktaş, 2012). Additionally, melamine adsorption to GAC can buffer variable influent concentrations, which is beneficial for biodegradation since it avoids peak loads and starvation conditions (Bourneuf et al., 2016; Li and Moe, 2005).

We propose that a combination of adsorption to GAC and biodegradation can be used to treat water containing melamine. Furthermore, melamine degrading biomass can be used to 
bioregenerate GAC loaded with melamine. To bioregenerate GAC, the optimal conditions for melamine biodegradation were studied. We assessed melamine biodegradation in fully oxic and anoxic conditions, as well as alternated oxic and anoxic conditions, with and without an additional carbon source. The most favourable conditions for melamine biodegradation were applied in a 3-step batch experiment to bioregenerate GAC loaded with melamine, and the bioregeneration extent was assessed. Our results provide additional insights in the optimal conditions for melamine biodegradation, GAC bioregeneration, and the limiting factors for GAC bioregeneration.

\subsection{Materials and methods}

\subsubsection{Chemicals}

Melamine 99\% purity (CAS 108-78-1) was purchased from Sigma Aldrich. Ammeline 90\% purity (CAS 108-78-1) was purchased from Alfa Aesar. Ammelide 99,4\% purity (CAS 10878-1) was purchased from Dr. Ehrenstofer GmbH. Cyanuric acid for synthesis (CAS 108-805) was purchased from Merck KGaA. Phosphate buffer for the mobile phase for liquid chromatography (LC) was prepared with $\mathrm{Na} 2 \mathrm{HPO} 4$ (Merck) and $\mathrm{NaH}_{2} \mathrm{PO}_{4} * 1 \mathrm{H}_{2} \mathrm{O}$ (Sigma Aldrich).

\subsubsection{Melamine degrading biomass}

Melamine degrading biomass in activated sludge was obtained from a WWTP in Romania treating industrial wastewater containing melamine at an average concentration of $58 \mathrm{mg} / \mathrm{L}$. The treatment includes nitrification and denitrification steps and uses methanol as carbon source. After being collected, the sludge was stored up to 2 months before being used. The sludge was collected at two moments, May 2018 and January 2020. The volatile suspended solids (VSS) in the first and second batch of sludge were 2.45 and $9.24 \mathrm{mg} / \mathrm{L}$ respectively. To obtain the desired VSS concentration for each experiment, the sludge was centrifuged and resuspended in media or buffer before use (media and buffer composition are given in Table S4.1).

\subsubsection{Melamine biodegradation in oxic or anoxic conditions}

Melamine biodegradation was tested in oxic and anoxic conditions, with and without methanol, in duplicates. Anoxic conditions were achieved in the bottles by flushing the media with nitrogen gas for 10 minutes and then exchanging the gas phase with nitrogen gas in 5 cycles using a gas exchanger. Batches contained $0.5 \mathrm{~mL}$ melamine degrading sludge centrifuged and resuspended in nitrogen free mineral media, $38 \mathrm{mg} / \mathrm{L}$ melamine and 
mineral media (Table S4.1) to a total liquid volume of $150 \mathrm{~mL}$ in $250 \mathrm{~mL}$ serum bottles. Methanol, when present, was spiked at $8.5 \mathrm{mg} / \mathrm{L}$. The VSS concentration in the batches was $51 \mathrm{mg} / \mathrm{L}$. Melamine and ammeline concentrations were analysed on days 0, 1, 3, 6, 8 and 10. Ammonium, nitrate and nitrite concentrations were analysed on day 6 and 10 .

\subsubsection{Effect of alternating redox conditions on melamine biodegradation}

The effect of alternating redox conditions and supply of additional carbon source on melamine biodegradation and nitrogen removal was assessed in a batch experiment. Melamine degradation was studied in 5 different conditions, as depicted in Table 4.1. Anoxic periods were applied in 3 of the 5 conditions to allow denitrification. Anoxic conditions were achieved as described in 2.3. To restore oxic conditions, the same procedure was done using compressed air instead of nitrogen gas. The inoculum used in this experiment consisted of melamine degrading biomass pre-cultured for 7 days in oxic and for further 6 days in anoxic conditions. Details about the inoculum are described in section S4.2.1.

Table 4.1: Melamine biodegradation test in alternating redox conditions.

\begin{tabular}{|c|c|c|c|c|c|c|}
\hline & & \multicolumn{5}{|c|}{ DAY } \\
\hline & & $0-3$ & $4-13$ & $14-20$ & $21-30$ & $31-39$ \\
\hline \multirow{5}{*}{ 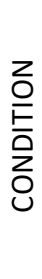 } & 1 & $\mathrm{MeOH}$ & $\mathrm{MeOH}$ & $\mathrm{MeOH}$ & $\mathrm{MeOH}$ & $\mathrm{MeOH}$ \\
\hline & 2 & & $\mathrm{MeOH}$ & & $\mathrm{MeOH}$ & \\
\hline & 3 & & & & & \\
\hline & 4 & $\mathrm{MeOH}$ & & $\mathrm{MeOH}$ & & $\mathrm{MeOH}$ \\
\hline & 5 & & & & & \\
\hline
\end{tabular}

Blue indicates oxic periods and red indicates anoxic periods; $\mathrm{MeOH}$ indicates addition of methanol.

Batches contained $10 \mathrm{~mL}$ of inoculum, $36 \mathrm{mg} / \mathrm{L}$ melamine $(24 \mathrm{mg}-\mathrm{N} / \mathrm{L})$ and nitrogen free mineral media (Table S4.1) to a total liquid volume of $150 \mathrm{~mL}$ in $250 \mathrm{~mL}$ serum bottles. The VSS concentration in the batches was $54 \mathrm{mg} / \mathrm{L}$. Methanol was added to bottles of conditions 1, 2 and 4 at different days as indicated in Table S4.2. At the start of the oxic periods (days 0,

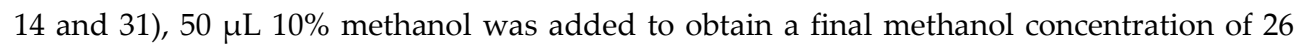
$\mathrm{mg} / \mathrm{L}$. In the anoxic periods, different volumes of $1 \%$ methanol was added to a final concentration of 10.5 to $15.8 \mathrm{mg} / \mathrm{L}$. The exact amount of methanol added at each day is given in Table S4.3. Samples for melamine, ammeline, nitrate and nitrite measurements were taken on days $0,2,4,7,14,18,21,28,31,35$, and 39. Ammonium samples were taken and directly analysed on days $0,4,14$ and 21 in one replicate of each condition. 


\subsubsection{Melamine adsorption and desorption isotherm}

Adsorption and desorption isotherms for melamine were obtained to: 1) assess reversibility of melamine adsorption, a requirement to bioregenerate loaded GAC; 2) determine isotherm coefficients so that melamine load on GAC in different steps of the bioregeneration process could be calculated. Eight different melamine initial concentrations were used, ranging from 8 to $387 \mathrm{mg} / \mathrm{L}$, in a total volume of $50 \mathrm{~mL}$ mineral media (Table S4.1). Relatively high melamine concentrations were used so that the initial concentration of the bioregeneration step would be within the concentration range of the isotherm. A mass of $0.05 \mathrm{~g}$ GAC was added to bottles with different melamine initial concentrations and mixed at $120 \mathrm{rpm}$ at $20^{\circ} \mathrm{C}$ for 21 days. AcquaSorbTM K-CS $\left(\mathrm{Jacobi}^{\circledR}\right)$ was used in our experiments, a coconut shell-based activated carbon, that is thermally activated and which has $96 \%$ of internal surface area as micropores (Piai et al., 2019). Before being used, the GAC was sieved to obtain particle sizes between 0.5 and $1 \mathrm{~mm}$ diameter.

Samples for equilibrium concentration were taken at day 21 and GAC was filtered from the solution using filter papers. GAC was dried for approximately $1 \mathrm{~h}$ and transferred to new bottles for desorption. These bottles contained $47 \mathrm{~mL}$ mineral media and $3 \mathrm{~mL}$ inactive biomass to a final VSS concentration of $77 \mathrm{mg} / \mathrm{L}$. The media had the same composition as in the adsorption step but without melamine. Inactive biomass consisted of melamine degrading sludge, centrifuged and resuspended in buffer (Table S4.1), inactivated by autoclaving it twice in consecutive days. Bottles were kept at $20^{\circ} \mathrm{C}$, mixed at $120 \mathrm{rpm}$ and desorption equilibrium samples were taken after 21 days to measure melamine concentration. A control without GAC and with melamine was done to verify that the inoculum was inactivated.

The Freundlich isotherm (Eq. 4.1) was used to fit the adsorption data using a non-linear optimization as suggested by Tran et al. (2017), starting from the linearized form of the model (Eq. 4.2)

$$
\begin{gathered}
q_{e}=K_{f, a d s} c_{e}{ }^{n} \\
\log q_{e}=n \log c_{e}+\log K_{f, a d s}
\end{gathered}
$$

where $K_{f, a d s}$ is the adsorption Freundlich constant $(\mathrm{L} / \mathrm{g}), c_{e}$ is the melamine concentration at equilibrium (mg/L), and $n$ is the Freundlich intensity parameter (dimensionless). 
Data from the adsorption isotherms were used to determine the coefficients $K_{f, a d s}$ and $n$. The $\mathrm{n}$ value obtained for the adsorption isotherm was used as input, together with the desorption data, to determine the desorption Freundlich constant $\left(K_{f, \text { des }}\right.$, in L/g) similar to the procedure followed by Aschermann et al. (2019b). The purpose of this approach is to compare adsorption and desorption affinities based on a single coefficient $\left(K_{f}\right)$.

\subsubsection{Bioregeneration of GAC loaded with melamine}

Melamine degrading biomass was used to bioregenerate GAC loaded with melamine in a 3step batch experiment as follows (Figure 4.2):

1) GAC loading: fresh GAC was loaded with melamine;

2) GAC bioregeneration: melamine degrading biomass was added to melamine loaded GAC;

3) GAC reloading (assessment of bioregeneration extent): bioregenerated GAC was reloaded to assess its remaining adsorption capacity and quantify the extent of bioregeneration.

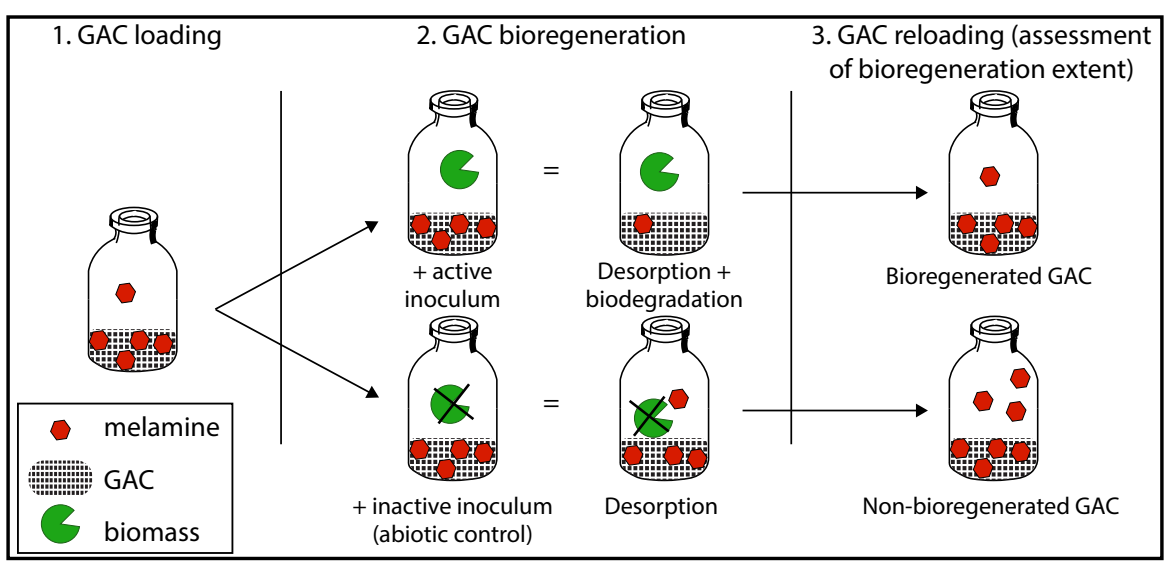

Figure 4.2: Schematic representation of steps in GAC bioregeneration experiment.

\subsubsection{GAC loading}

In the GAC loading step, $0.15 \mathrm{~g}$ GAC was used to adsorb melamine at an initial concentration of $246 \mathrm{mg} / \mathrm{L}$ in $150 \mathrm{~mL}$ of mineral media (Table S4.1). This relatively high melamine concentration was used so that the melamine load on GAC would be close to saturation levels and the effect of bioregeneration would be detectable. Bottles containing GAC, melamine and mineral media were mixed at $120 \mathrm{rpm}$ at $20^{\circ} \mathrm{C}$ for 7 days. Liquid 
samples $(3 \mathrm{~mL})$ were taken every hour between the beginning of the experiment and $4.5 \mathrm{~h}$, and once a day on days 1,2, 3, 6 and 7. A total of 4 replicate bottles were prepared and sampled either every hour until $4.5 \mathrm{~h}$ or daily until day 7 to avoid subtracting more than $10 \%$ of liquid volume by sampling during the adsorption periods.

\subsubsection{GAC bioregeneration}

After the loading step, GAC was separated from the solution using filter paper, dried for $1 \mathrm{~h}$ and transferred to a clean bottle containing $140 \mathrm{~mL}$ mineral media (Table S4.1) spiked with $36 \mu \mathrm{L}$ methanol and $10 \mathrm{~mL}$ melamine degrading biomass centrifuged and resuspended in buffer (Table S4.1). The VSS concentration in the bottles was $82 \mathrm{mg} / \mathrm{L}$. Pure methanol was added in fractionated doses $(6 \mu \mathrm{L}$ each dose) between days 0 and 6 , to avoid methanol toxicity. An abiotic control was taken along, with autoclaved biomass as inoculum. Methanol was added to the control only on the first day and demineralized water was added in the following days to keep the total liquid volume the same in test and control conditions.

After 7 days of incubation under oxic conditions, head space was replaced by $100 \% \mathrm{~N}_{2}$ to

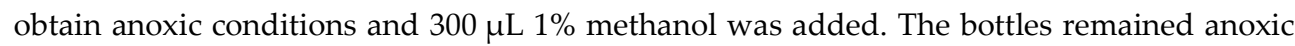
for 7 days. After the bioregeneration period (14 days), liquid samples were taken from the biologically active bottle and abiotic control for measuring melamine concentration, followed by autoclaving the bottles and cooling. GAC was separated from the solution using filter paper and added to bottles with fresh medium with melamine to reload the GAC and assess the extent of bioregeneration in the third step of our experiment (4.2.6.3).

Residual melamine load on GAC (in $\mathrm{mg} / \mathrm{g}$ ) for the bioregenerated GAC $\left(q_{0,2 b i o}\right)$ and abiotic control $\left(q_{0,2 a b i o}\right)$ at the end of this step were calculated based on the melamine concentration in the liquid phase in each treatment $\left(c_{0,2 b i o}\right.$ and $c_{0,2 a b i o}$ in $\left.\mathrm{mg} / \mathrm{L}\right)$ and by using the desorption isotherm coefficients (see 4.2.5), according to Eq. 4.3.

$$
q_{0,2(a) b i o}=K_{f, d e s} c_{0,2(a) b i o}{ }^{n}
$$

\subsubsection{GAC reloading (assessment of bioregeneration extent)}

To assess the extent of bioregeneration, bioregenerated GAC and the abiotic control were reloaded at the same conditions as the GAC loading step, i.e., $150 \mathrm{~mL}$ mineral media, 251 $\mathrm{mg} / \mathrm{L}$ melamine and the full amount of GAC from step 2. Bottles were sampled at the same time points as in the first GAC loading step (4.2.6.1). 
Melamine concentration on day 7 was used to calculate melamine load on GAC which we assumed to be at equilibrium, based on the melamine mass balance, according to Eq. 4.4

$$
q_{e, i}=\frac{\left(c_{0, i}-c_{e, i}\right) v}{m}
$$

where $q_{e, i}(\mathrm{mg} / \mathrm{g})$ is the melamine load on GAC at equilibrium (day 7$), c_{0, i}(\mathrm{mg} / \mathrm{L})$ is the initial melamine concentration, $c_{e, i}(\mathrm{mg} / \mathrm{L})$ is the melamine concentration at equilibrium (day 7), $v(\mathrm{~L})$ is the solution volume and $m(\mathrm{~g})$ is the GAC mass. The index $i$ refers either to the GAC loading (1) or reloading (2bio and 2abio) steps.

Bioregeneration efficiency (\%) was determined by subtracting the adsorption capacity of the abiotic control from the adsorption capacity of the bioregenerated GAC, normalized on the adsorption capacity of the abiotic control, according to Eq. 4.5:

$$
\text { Bioregeneration efficiency }(\%)=\frac{q_{e, 2 b i o}-q_{e, 2 a b i o}}{q_{e, 2 a b i o}} \times 100
$$

where $q_{e, 2 b i o}$ and $q_{e, 2 a b i o}$ are the adsorption capacity of the bioregenerated GAC and the abiotic control on day 7 of the reloading step, based on the mass balance as described in Eq. 4.4 .

Total melamine load on GAC after reloading was calculated for the bioregenerated GAC and the abiotic control, according to Eq. 4.6.

$$
q_{\text {total }}=q_{e, 2(a) b i o}+q_{0,2(a) b i o}
$$

Eq. 4.6

where $q_{\text {total }}(\mathrm{mg} / \mathrm{g})$ is the total melamine load on GAC after the reloading step.

\subsubsection{Adsorption kinetics}

A pseudo-first-order kinetic equation, as described by Ho and McKay (1998) (Eq. 4.7), was used to fit the adsorption data and compare the adsorption rates of fresh GAC, bioregenerated GAC and abiotic control. We used a non-linear optimization as suggested by Tran et al. (2017), starting from the linearized form of the equation (Eq. 4.8)

$$
\begin{array}{cc}
q_{t, i}=q_{e, i}\left(1-e^{-k_{1} t}\right) & \text { Eq. } 4.7 \\
\ln \left(q_{e, i}-q_{t, i}\right)=-k_{1} t+\ln \left(q_{e, i}\right) & \text { Eq. } 4.8
\end{array}
$$


where $q_{t, i}(\mathrm{mg} / \mathrm{g})$ is the amount of melamine adsorbed at time $t(\mathrm{~h}), q_{e, i}(\mathrm{mg} / \mathrm{g})$ is the amount of melamine adsorbed at equilibrium (day 7$)$ and $k_{1}(1 / \mathrm{h})$ is the adsorption rate constant. The index $i$ refers either to the GAC loading (1) or reloading (2bio and 2abio) steps.

\subsubsection{Chemical analysis}

All liquid samples were filtered with $0.2 \mu \mathrm{M}$ polyethersulfone membrane filters and either analysed immediately (for ammonium), within 7 days (nitrate and nitrite) or stored at $-20^{\circ} \mathrm{C}$ until analysis (for melamine and ammeline).

Melamine and ammeline concentrations were measured by LC coupled to UV-detection. In the same system, ammelide and cyanuric acid were detected but could not be separated and, therefore, could not be quantified. The LC consisted of a Ultimate 3000 coupled to a 4 channel UV detector (Dionex) and the compounds were detected at $220 \mathrm{~nm}$. First, a method was used in which sample volumes of $50 \mu \mathrm{L}$ were injected onto a Luna Omega column $(150 \mathrm{~mm} \times 4.6 \mathrm{~mm}, 3 \mu \mathrm{m})$ maintained at $40^{\circ} \mathrm{C}$. The compounds were separated using gradient elution with a flow of $1 \mathrm{~mL} / \mathrm{min}$. Solvents were (A): water/ammonium formate $2 \mathrm{mM} /$ formic acid $0.016 \%(\mathrm{v} / \mathrm{v})$ and (B): methanol/ammonium formate $2 \mathrm{mM} /$ formic acid $0.016 \%(\mathrm{v} / \mathrm{v})$. The gradient applied was: 0-3 min linearly increased to $100 \% \mathrm{~B}, 3-5 \mathrm{~min}$ stable at $100 \% \mathrm{~B}$, decreased in $2 \mathrm{~min}$ to $0 \% \mathrm{~B}$ and stable at this condition until $8 \mathrm{~min}$. Thereafter, the method was changed and sample volumes of $5 \mu \mathrm{L}$ were injected onto a Luna CN column $(250 \mathrm{~mm} \times 4.6 \mathrm{~mm}, 3 \mu \mathrm{m})$ maintained at $35^{\circ} \mathrm{C}$. Compounds were separated using isocratic elution of sodium phosphate buffer $5 \mathrm{mM}$ at $\mathrm{pH}$ 6.7.

Nitrate and nitrite were measured using ion chromatography, as described in Wagner et al. (2020). Ammonium was measured using Hach Lange colorimetric kits (LCK 303 and LCK 305) and a spectrophotometer (Hach Lange DR 3900).

\subsection{Results and discussion}

\subsubsection{Melamine biodegradation in oxic and anoxic conditions}

Figure 4.3a shows that melamine was biodegraded in both oxic and anoxic conditions and that the biodegradation rate was faster in the latter. Ammeline, the first degradation product of melamine, was also degraded faster in anoxic conditions (Figure 4.3b). Ammelide and/or cyanuric acid were still present in oxic bottles on day 10 whereas they were not detected in anoxic bottles on that day (data not shown). Methanol did not increase the melamine biodegradation rate. 

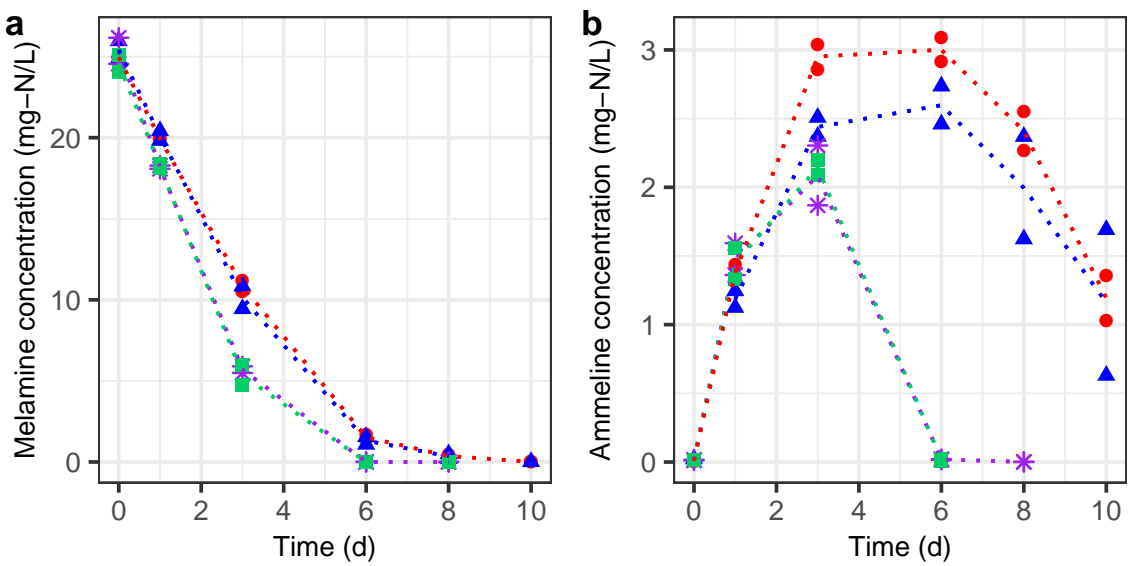

$-\_$oxic $\mathrm{MeOH}-\bullet$ oxic $-\div$ anoxic $\mathrm{MeOH}-*$ anoxic

Figure 4.3: Melamine (a) and ammeline (b) concentrations in oxic and anoxic conditions, with and without methanol. Duplicates are plotted individually and lines connect the mean value of the duplicates in each time point.

The addition of methanol mainly affected the nitrification rate, rather than the melamine biodegradation rate. In the oxic bottles with and without methanol, $36 \%$ or more of the nitrogen was still present as ammonium on day 6 (Figure 4.4a). The accumulation of ammonium shows that nitrification rate was slower than melamine biodegradation rate. In the oxic bottles without methanol, $85 \%$ of the nitrogen from melamine was converted to nitrate in 10 days. When methanol was present, less than $45 \%$ of nitrogen was converted to nitrate (Figure $4.4 \mathrm{~b}$ ). 

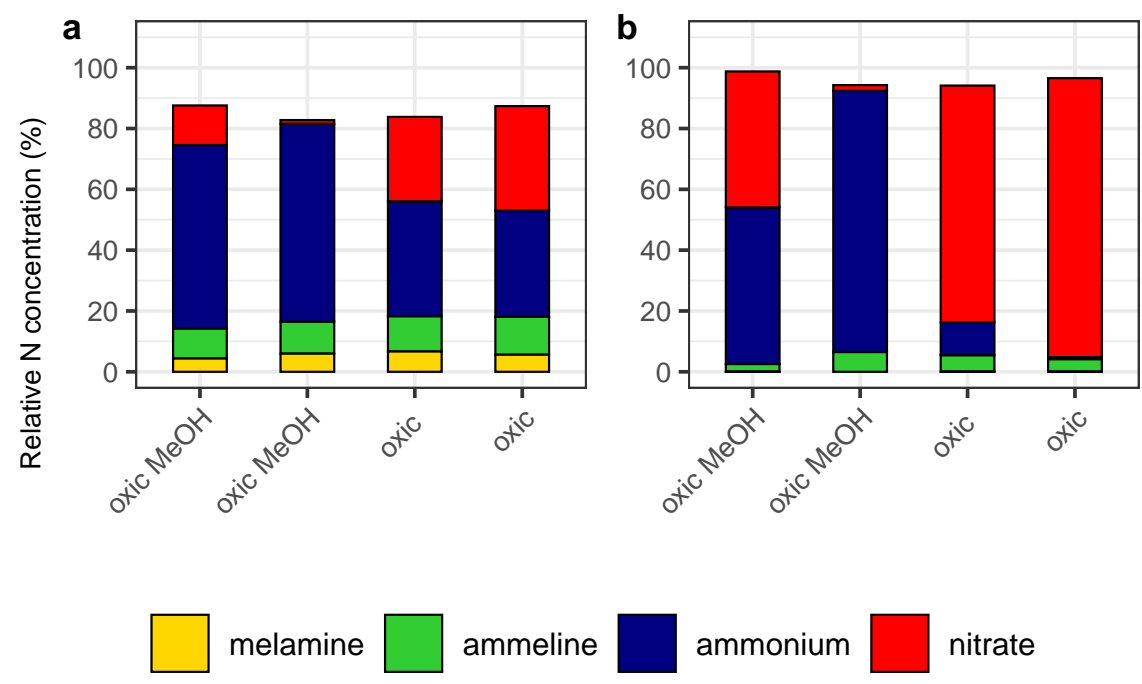

Figure 4.4: Relative nitrogen concentration in melamine, ammeline, nitrate and ammonium in melamine biodegradation test, based on melamine as sole nitrogen source. Both duplicates are shown. a: day 6; b: day 10.

The negative effect of methanol on nitrification rate is not fully understood. First, methanol did not result in a limiting oxygen concentration, as presented by the oxygen concentrations in our batches. Sufficient oxygen ( $>14 \%$ ) was present in the oxic bottles with and without methanol (Table S4.4). Furthermore, nitrite was not detected in any of the samples, also indicating that oxygen did not limit nitrification. As nitrite oxidation is affected by low dissolved oxygen levels to a larger extent than ammonium oxidation (Soliman and Eldyasti, 2018), nitrite would have accumulated in the media if oxygen had been limiting in our batches. Second, methanol can bind to the ammonium mono-oxygenase, one of the enzymes involved in nitrification, and hence inhibit it (McBride et al., 2019). Inhibition of nitrification by methanol has been reported in literature (Jönsson et al., 2001; Martin and Richard, 1982; Suzuki et al., 1976; Voysey and Wood, 1987), although most studies report inhibitory effects at levels at least 10 times higher than the initial concentration used in this experiment (8.5 $\mathrm{mg} / \mathrm{L}$ ). We did not expect toxic effects from methanol at this concentration, given that methanol is used as carbon source for denitrification in the WWTP where the sludge originated in doses up to $400 \mathrm{mg} / \mathrm{L}$. Therefore, the observed inhibition of nitrification in the presence of methanol requires further investigation.

Our results show that melamine can be biodegraded in both oxic and anoxic conditions, which is in agreement with previous studies. Most melamine biodegradation studies have 
been performed in oxic conditions (El-Sayed et al., 2006; Shiomi and Ako, 2012; Takagi et al., 2012; Wang et al., 2014) and the study of Jutzi et al. (1982) is the sole report of melamine biodegradation in anoxic conditions.

\subsubsection{Effect of alternating redox conditions on melamine biodegradation}

In a follow-up experiment, we assessed melamine biodegradation in alternating oxic and anoxic conditions. Melamine was biodegraded to a larger extent when oxic and anoxic conditions were alternated, compared to maintaining oxic conditions throughout the incubation period (Figure 4.5). Comparing alternating redox conditions, melamine was degraded faster when methanol was supplied in both oxic and anoxic periods (Figure 4.5a). When oxic conditions were maintained throughout the experiment, the addition of methanol did not increase the melamine biodegradation rate (Figure $4.5 b$ ).
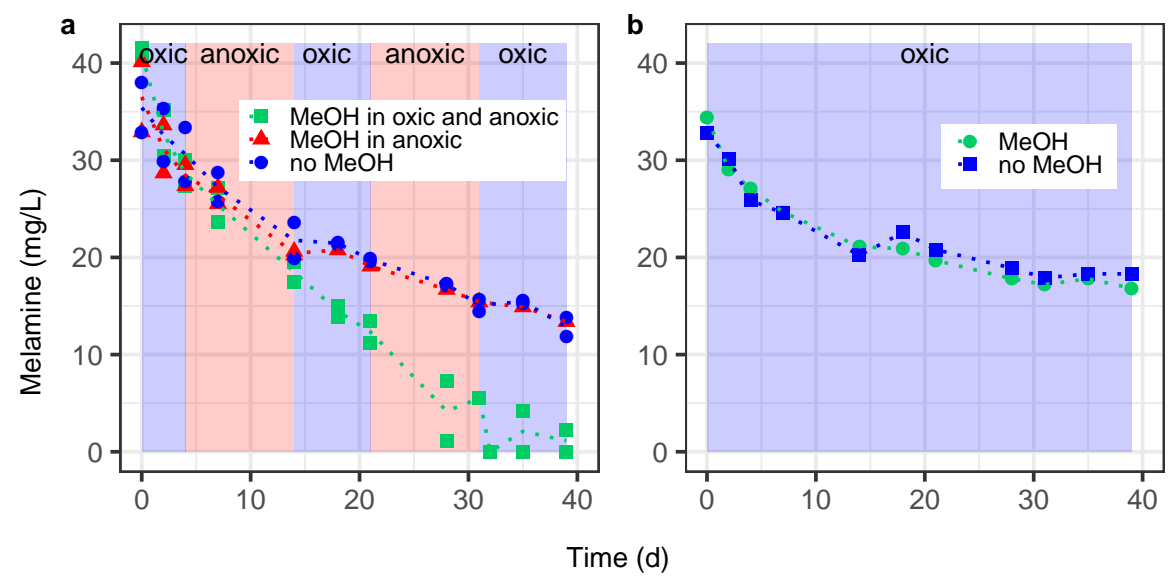

Figure 4.5: Melamine concentration in biodegradation experiment. a: alternating oxic (blue background) and anoxic (red background) conditions. b: oxic conditions. For details of experimental setup, see Table 4.1. In plot a, duplicates are plotted individually and lines connect the mean value of the duplicates in each time point.

Ammonium did not accumulate in the batches, neither in the oxic, nor in the anoxic periods (Table S4.5), differently from our results when only oxic or only anoxic conditions (see 4.3.1) were applied, and up to $43 \mathrm{mg}$ NH4-N/L accumulated. In those oxic conditions, melamine degradation rate was $4.9 \mathrm{mg}-\mathrm{N} / \mathrm{L} . \mathrm{d}$ in the first 3 days (4.3.1, Figure 4.3), whereas melamine degradation rate was $1.3 \mathrm{mg}-\mathrm{N} / \mathrm{L} . \mathrm{d}$ in the first 4 days in the experiment with alternating conditions (Figure 4.5). This shows a difference in melamine biodegradation rates in the first days in oxic conditions, despite their similar set-up. The lower degradation rate was likely a result of the applied pre-culturing period. As a result, nitrification was the rate limiting step 
for nitrate production in 4.3.1, resulting in accumulation of ammonium in the medium, whereas melamine degradation was the rate limiting step for nitrate production in this experiment.

Ammonium accumulation was expected in the anoxic periods, when ammonium is produced from the degraded melamine, and nitrification does not occur due to the lack of oxygen. Considering the stoichiometry of melamine degradation and ammeline production in the first anoxic period (days 4 to 14 ), 1.0 to $4.6 \mathrm{mg}-\mathrm{N} / \mathrm{L}$ of ammonium should be produced. However, this stoichiometry was not reached, as ammonium concentration in all batches in this period remained below $0.05 \mathrm{mg}-\mathrm{N} / \mathrm{L}$ (Table S4.5). No nitrite was detected in this period, confirming that partial nitrification did not occur. The fate of the nitrogen released from melamine in the anoxic periods in this experiment remains to be clarified.

When methanol was supplied in the anoxic period, the nitrate produced in the oxic period was removed by denitrification (Figure 4.6a and S4.1a). Without methanol in the anoxic period, the nitrate concentration decreased slightly between days 21 and 30 (Figure 4.6b). The decrease in nitrate concentration indicates that the carbon present in melamine becomes available when the aromatic ring is hydrolysed, and is used for denitrification. The denitrification rate in that case is much lower compared to denitrification with methanol. Furthermore, methanol had a negative impact on nitrification rates in this experiment, like in our experiments with either only oxic or anoxic conditions (4.3.1). This is shown by the average nitrate concentration on day 4 , which was $20 \%$ lower in bottles with methanol, compared to bottles where no methanol was added. Overall, the results indicate that methanol increases melamine biodegradation rate when nitrogen limiting conditions are created, despite the negative influence of methanol on nitrification rates.

Considering our results on the effect of redox conditions and additional carbon source on melamine biodegradation, we conclude that the most favourable conditions for melamine biodegradation are created when nitrogen is removed from the media and methanol is supplied in both oxic and anoxic periods. Therefore, these conditions were applied to bioregenerate GAC loaded with melamine. 

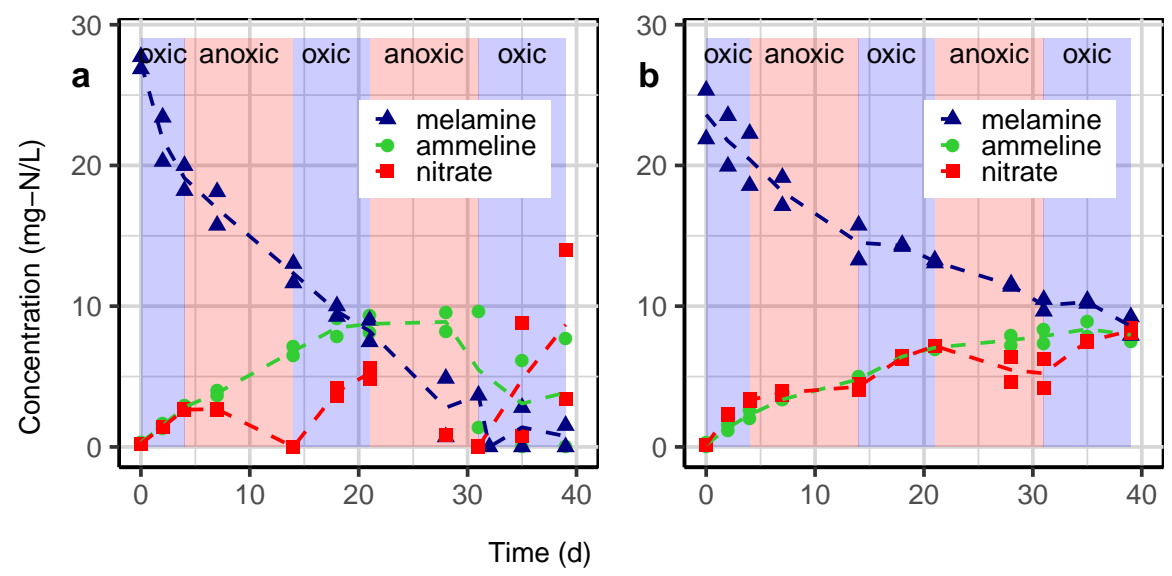

Figure 4.6: Melamine, ammeline and nitrate concentrations during melamine biodegradation. a: alternating oxic and anoxic conditions with methanol in both periods. $b$ : alternating oxic and anoxic conditions without methanol. Duplicates are plotted individually and lines connect the mean value of the duplicates in each time point.

\subsubsection{Bioregeneration of GAC loaded with melamine}

\subsubsection{Reversibility of melamine adsorption}

Melamine adsorption and desorption isotherms were fit with the Freundlich model to assess reversibility of melamine adsorption (Figure S4.2). The calculated Freundlich constant for adsorption $\left(K_{f, a d s}\right)$ and desorption $\left(K_{f, d e s}\right)$ were 30.4 and $30.8 \mathrm{~L} / \mathrm{g}$ respectively. The similar values of $K_{f, a d s}$ and $K_{f, \text { des }}$ indicate that melamine adsorption is a fully reversible process on the conditions tested, i.e., in the presence of inactive biomass.

\subsubsection{GAC bioregeneration}

Bioregeneration was achieved by mixing loaded GAC with melamine degrading biomass (Figure 4.2, step 2). The average melamine concentration at the end of the bioregeneration, i.e. after 14 days, was $0.6 \mathrm{mg} / \mathrm{L}$ in the biologically active bottles and $46 \mathrm{mg} / \mathrm{L}$ in the abiotic controls. These concentrations were used to calculate the melamine load on GAC at the end of the bioregeneration step $\left(q_{0,2 b i o}\right.$ and $\left.q_{0,2 a b i o}\right)$, by applying the desorption isotherm coefficients, according to Eq. 4.3 (Table 4.2). 
Table 4.2: Melamine concentration and calculated melamine load on GAC at the end of the GAC bioregeneration (step $2^{*}$ ) in the bioregeneration experiment (Figure. 4.2).

\begin{tabular}{lrr}
\hline & $c_{0,2}(\mathrm{mg} / \mathrm{L})$ & $q_{0,2} * *(\mathrm{mg} / \mathrm{g})$ \\
\hline Biologically active & 0.6 & 23 \\
Abiotic control & 46 & 134 \\
\hline
\end{tabular}

* See Figure 4.2 for the experimental steps.

** Based on Freundlich isotherm (Eq. 4.3).

Since melamine concentration was much lower in the biologically active bottles than in the controls, we decided to proceed to the next step, i.e., reloading the GAC, instead of applying a second oxic period.

After bioregeneration, biomass was inactivated and the remaining adsorption capacity of the GAC was assessed reloading the treated GAC. The extent of bioregeneration in this study was assessed by measuring the adsorption capacity of the bioregenerated GAC compared to the abiotic control which was exposed to inactive biomass (see also Figure 4.2, step 3). The adsorption capacity of bioregenerated GAC at equilibrium was higher than of the abiotic control but lower than of the fresh GAC (Table 4.3).

Table 4.3: Amount of melamine adsorbed by fresh GAC in the loading step and by bioregenerated GAC and abiotic control in the reloading step.

\begin{tabular}{llrrr}
\hline GAC & Experimental step & $c_{0, i}(\mathrm{mg} / \mathrm{L})$ & $c_{e, i}(\mathrm{mg} / \mathrm{L})$ & $q_{e, i}^{* *}(\mathrm{mg} / \mathrm{g})$ \\
\hline Fresh & 1 (GAC loading) & 246 & 79 & 166 \\
Bioregenerated & 3 (GAC reloading) & 251 & 128 & 124 \\
Abiotic control & 3 (GAC reloading) & 251 & 154 & 97 \\
\hline
\end{tabular}

* See Figure 4.2 for the experimental steps.

** Based on mass balance (Eq. 4.4).

A bioregeneration efficiency of $28 \%$ was calculated (Eq. 4.5), which is lower than reported bioregeneration efficiencies for GAC loaded with phenolic compounds (Oh et al., 2011), but comparable to efficiencies obtained in batch systems for GAC loaded with surfactants (Klimenko et al., 2003). This relatively low bioregeneration efficiency in our study is partially due to the high adsorption capacity of the abiotic control when reloaded $(97 \mathrm{mg} / \mathrm{g})$. The high adsorption capacity of the abiotic control shows that significant regeneration was achieved as a consequence of the experimental design, i.e., simply by transferring the GAC to a solution without melamine (second experimental step). These results also show that bioregeneration efficiency cannot be assessed in our study by comparing the adsorption 
capacity of bioregenerated and fresh GAC, as recommended by Narbaitz and Cen (1997), otherwise unrealistic high efficiencies would be calculated.

\subsubsection{Limiting factors for bioregeneration}

We compared the total melamine load on GAC after reloading ( $q_{\text {total }}$, Eq. 4.6), to the expected load based on the melamine concentration in the liquid phase at the end of the reloading step and the adsorption isotherm coefficients. We also compared the melamine load on fresh GAC $\left(q_{e, 1}\right)$ to the expected load based on the adsorption isotherm. Results show that for the fresh GAC and the abiotic control, the total melamine load on GAC matches the expected load based on the adsorption isotherm (Figure 4.7). For bioregenerated GAC, the total melamine load on GAC is lower (27\%) than the expected load based on the adsorption isotherm, indicating that not all adsorption capacity could be regenerated.

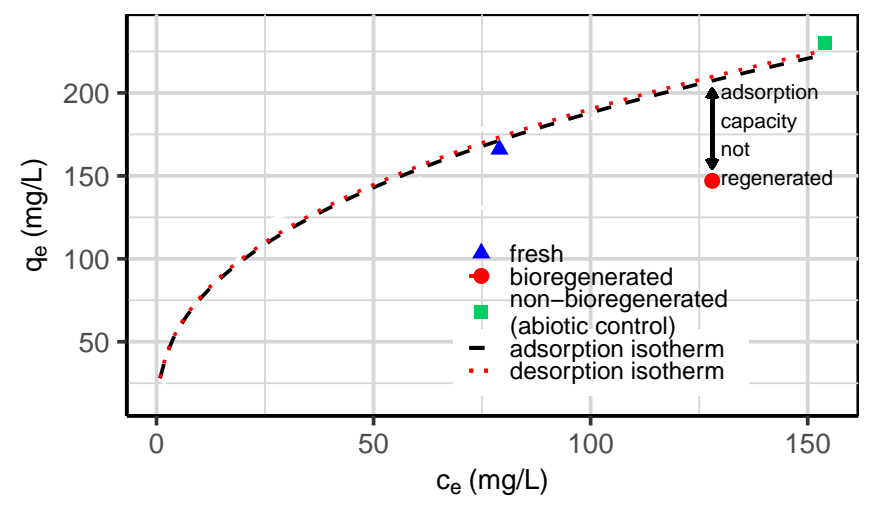

Figure 4.7: Melamine load on fresh GAC, bioregenerated GAC and abiotic control compared to the expected load based on the adsorption and desorption isotherms.

A possible explanation for the unexpected lower adsorption capacity of bioregenerated GAC is related to the adsorption of intermediate products of melamine biodegradation (ammeline, ammelide and cyanuric acid). These compounds were not detected in the end of the bioregeneration step and did not accumulate in any of the melamine biodegradation experiments. However, we may assume that melamine degradation products were present at concentrations below the detection limits of the analytical method, hence a fraction of them was adsorbed. Such a fraction would use adsorption sites in the GAC, thus contributing to the lower availability of adsorption sites for melamine. 
Additionally, the reduced adsorption capacity of the bioregenerated GAC can also be related to the release of melamine previously adsorbed. The release of adsorbed melamine may be hindered by the presence of dissolved (and adsorbed) organic matter due to pore blockage, which would lead to desorption hysteresis (Aschermann et al., 2019a, 2019b). This effect is stronger in microporous GACs (Aschermann et al., 2019b), as is the case for the GAC used in this experiment (Piai et al., 2019). Our desorption isotherm shows that melamine adsorption was fully reversible in the presence of inactivated melamine degrading biomass (Figures 4.7 and S4.2). However, in the presence of active biomass, as used in our GAC bioregeneration experiment, organic molecules, products of microbial activity, can hinder desorption by causing pore blockage (Smolin et al., 2020). These organic molecules produced by the biomass can also have decreased bioregeneration efficiency by competing with melamine for adsorption sites in the GAC reloading step. Likely, these factors combined contributed to limiting the extent of bioregeneration. Nevertheless our results clearly show that bioregeneration can partially restore GAC adsorption capacity.

\subsubsection{Melamine adsorption rate}

The adsorption rate of melamine to fresh GAC, to bioregenerated GAC and to the abiotic control was assessed to evaluate the effect of GAC bioregeneration on adsorption kinetics. Similar adsorption kinetics would indicate if adsorption sites in all pore sizes are subject to bioregeneration to the same extent. In the first $2.5 \mathrm{~h}$, bioregenerated GAC adsorbed melamine to a similar extent as fresh GAC (Figure 4.8a). After this period, the adsorption capacity of bioregenerated GAC started to diverge from the fresh GAC. After 7 days the bioregenerated GAC showed an adsorption capacity that was intermediate between fresh GAC and non-bioregenerated GAC (Figure 4.8b). The effect of bioregeneration on the adsorption rate of each GAC in the first hours and after 7 days can also be observed by comparing the first-order adsorption rate constants $\left(k_{1}\right)$ of fresh, bioregenerated and nonbioregenerated GAC (Figure 4.8). The values of $k_{1}$ are similar for bioregenerated and fresh GAC and more than 3 times higher than for non-bioregenerated GAC in the first 4.5 hours of adsorption. However, the differences are smaller (less than double) when comparing the adsorption rates of all GACs in a period of 7 days. These results show that melamine biodegradation could restore part of the GAC adsorptive capacity, with a higher impact on adsorption rate than on the total adsorption capacity. 

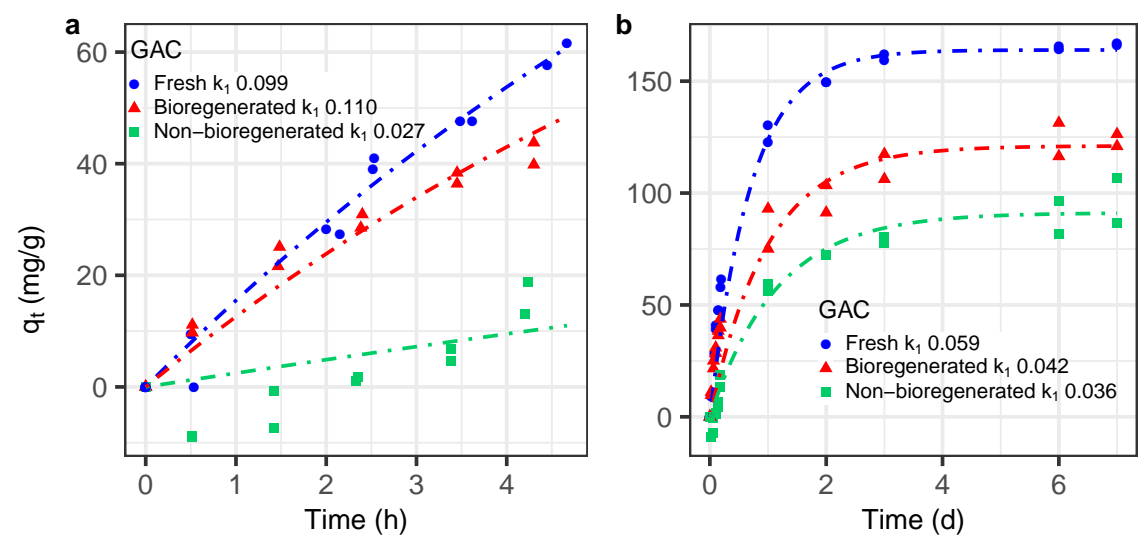

Figure 4.8: Melamine adsorption by fresh and bioregenerated and abiotic control and respective firstorder adsorption constant $\left(k_{1}\right.$ in $\left.1 / \mathrm{h}\right)$ in the period. a: First 4.5 hours of adsorption. b: 7 days of adsorption. Data points represent experimental data. Lines represented fitted pseudo-first order kinetics equation (Eq. 4.7).

The faster adsorption observed for the bioregenerated GAC compared to nonbioregenerated GAC supports the hypothesis of melamine desorption hysteresis due to pore blockage. Pore blockage is likely to happen in the smallest pore fraction of the GAC (Aschermann et al., 2019b). This means that melamine only desorbs from adsorption sites in the larger pore fraction and hence those are the pores subject to bioregeneration. Consequently, bioregenerated adsorption sites can be rapidly reached in the second adsorption test, reflecting in faster adsorption rates in bioregenerated GAC compared to non-bioregerenated GAC. These results have also been demonstrated in other modelling and experimental studies. Roy et al. (1999) modelled adsorption kinetics and calculated an intra-particle diffusion coefficient (Ds) for bioregenerated and fresh GAC, obtaining a higher Ds value for bioregenerated GAC. Furthermore, studies showed that the deepest layers of GAC loaded with surfactants or nitrophenol were not bioregenerated and most bioregeneration took only place in the mesopore fraction (Klimenko et al. 2003; Smolin et al. 2020). In our study, we provide additional evidence that bioregeneration happens mainly in the larger and more easily accessible pore fraction of the GAC. 


\subsection{Conclusions}

Our results show that melamine is biodegraded in oxic and anoxic conditions. In addition, alternating oxic and anoxic conditions promote further melamine biodegradation when an additional carbon source is present, as nitrogen limiting conditions are created and melamine is used as a nitrogen source.

Supply of an additional carbon source and alternating redox conditions were successfully applied to bioregenerate GAC preloaded with melamine. A 3 step-batch experiment was used to allow dividing the processes (GAC loading, bioregeneration and reloading) in different steps and calculate the extent of bioregeneration. In practice, GAC is often used in fixed- or fluidized-bed filters (Worch, 2012), where bioregeneration can be achieved in situ.

Bioregeneration partly restored the GAC's original adsorption capacity. The effect of bioregeneration is more pronounced in the first hours of adsorption. Bioregenerated GAC adsorbed almost as much melamine as fresh GAC in the first 4.5 hours of adsorption, and after this period, the bioregenerated GAC showed less adsorption capacity than the fresh GAC. This indicates that the regenerated adsorption sites are mainly the ones present in the largest and more quickly accessed pore fraction of the GAC.

To conclude, we have shown that the use of biomass capable of degrading micropollutants has the potential to extend the lifetime of GAC, hence reducing the need for thermal GAC reactivation. Knowledge on optimal conditions for biodegradation of micropollutants allows designing bioregeneration processes and avoids GAC thermal reactivation.

\section{Acknowledgements}

This work was financially supported by Evides Water Company N.V. (Rotterdam, The Netherlands). We thank Nijhuis Water Technology B.V. (Doetinchem, The Netherlands) for providing the melamine degrading sludge used in the experiments. We also thank Vanessa Afroedie for executing part of the experiments and Livio Carlucci, Jean Slangen and Julian Zamudio Pineres for developing the analytical method used to measure melamine and ammeline. Finally, we thank Andrea Aldas-Vargas, Koen van Gijn and Thomas Wagner for proof reading the manuscript. 


\section{Supplementary information to Chapter 4}

\section{S4.1 Media composition}

Table S4.1: Composition of media used in the experiments.

\section{Concentration (g/L)}

\begin{tabular}{|c|c|}
\hline Buffer & \\
\hline $\mathrm{Na}_{2} \mathrm{HPO}_{4} .2 \mathrm{H}_{2} \mathrm{O}$ & 1.78 \\
\hline $\mathrm{KH}_{2} \mathrm{PO}_{4}$ & 1.36 \\
\hline \multicolumn{2}{|l|}{ Macro nutrients } \\
\hline $\mathrm{CaCl}_{2} .2 \mathrm{H}_{2} \mathrm{O}$ & $4.8^{*} \times 10^{-2}$ \\
\hline $\mathrm{MgSO}_{4.7 \mathrm{H}_{2} \mathrm{O}}$ & $5.4 * 10^{-2}$ \\
\hline \multicolumn{2}{|l|}{ Trace elements } \\
\hline $\mathrm{FeCl}_{2} .4 \mathrm{H}_{2} \mathrm{O}$ & $1.2 * 10^{-3}$ \\
\hline $\mathrm{CoCl}_{2} .6 \mathrm{H}_{2} \mathrm{O}$ & $1.2 * 10^{-3}$ \\
\hline $\mathrm{MnCl}_{2} .4 \mathrm{H}_{2} \mathrm{O}$ & $3 * 10^{-4}$ \\
\hline $\mathrm{CuCl}_{2} .2 \mathrm{H}_{2} \mathrm{O}$ & $1.8 * 10^{-5}$ \\
\hline $\mathrm{ZnCl}_{2}$ & $3 * 10^{-5}$ \\
\hline $\mathrm{HBO}_{3}$ & $3 * 10^{-5}$ \\
\hline$\left(\mathrm{NH}_{4}\right)_{6} \mathrm{Mo}_{7} \mathrm{O}_{24} 4 \mathrm{H}_{2} \mathrm{O}$ & $5.4 \times 10^{-5}$ \\
\hline $\mathrm{Na}_{2} \mathrm{SeO}_{3} .5 \mathrm{H}_{2} \mathrm{O}$ & $6 * 10^{-5}$ \\
\hline $\mathrm{NiCl}_{2} .6 \mathrm{H}_{2} \mathrm{O}$ & $3 * 10^{-5}$ \\
\hline EDTA (tripex 2) & $6 * 10^{-4}$ \\
\hline Na-Resazurin & $3 * 10^{-4}$ \\
\hline $\mathrm{HCl} 36 \%$ & $7.2 * 10^{-4}$ \\
\hline
\end{tabular}

S4.2 Effect of alternating redox conditions on melamine biodegradation Materials and methods

\section{S4.2.1 Inoculum}

A volume of $10 \mathrm{~mL}$ melamine degrading sludge, centrifuged and resuspended in media was used to inoculate batches with a total working volume of $150 \mathrm{~mL}$. On day 7, acetate was added to a concentration of $85 \mathrm{mg} / \mathrm{L}$ and the gas phase was replaced by $100 \% \mathrm{~N}_{2}$ to allow for 
denitrification. On day 13, the bottle was stored at $4^{\circ} \mathrm{C}$. After 18 days of storage, this batch was used to inoculate the experiment described in 2.4.

\section{S4.2.2 Experimental conditions}

Table S4.2: Detailed experimental conditions of melamine biodegradation test in alternating redox conditions.

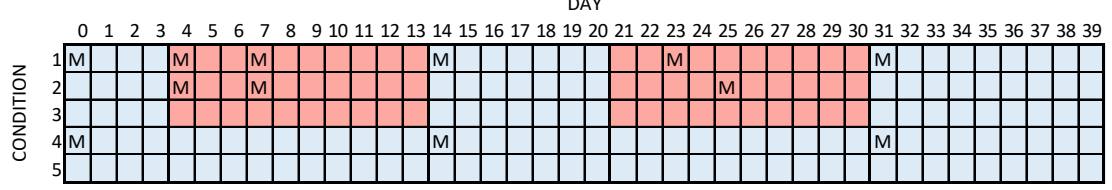

Blue indicates oxic periods and red indicates anoxic periods; $M$ indicates addition of methanol.

Table S4.3: Volume and concentration of methanol added to bottles of experiment described in 4.2.4.

\begin{tabular}{rrrr}
\hline Day & Condition & $\begin{array}{r}\text { Volume methanol } \\
\text { stock }(\mu \mathrm{L})\end{array}$ & $\begin{array}{r}\text { Concentration } \\
\text { methanol stock (\%) }\end{array}$ \\
\hline 0 & 1,4 & 50 & 10 \\
4 & 1,2 & 250 & 1 \\
7 & 1 & 200 & 1 \\
7 & 2 & 275 & 1 \\
14 & 1,4 & 50 & 10 \\
23 & 1 & 300 & 1 \\
25 & 2 & 250 & 1 \\
31 & 1,4 & 50 & 10 \\
\hline
\end{tabular}

Blue indicates oxic periods and red indicates anoxic periods.

\section{S4.3 Melamine biodegradation in oxic and anoxic conditions}

Table S4.4: Oxygen concentration (\%) in gas phase of melamine degrading batches in fully oxic or anoxic conditions.

\begin{tabular}{lrrrr} 
& \multicolumn{5}{c}{ Day } \\
\hline Bottle & 0 & 2 & 7 & 10 \\
\hline Oxic MeOH_1 & 20.7 & 19.0 & 16.5 & 14.2 \\
Oxic MeOH_2 & n.m. & n.m. & 17.9 & 17.6 \\
Oxic_1 & n.m. & n.m. & 16.5 & 13.1 \\
Oxic_2 & n.m. & n.m. & n.m. & 12.1 \\
Anoxic $\mathrm{MeOH}_{1}$ 1 & 0.4 & 0.2 & 0.2 & n.m. \\
Anoxic MeOH_2 & 0.2 & n.m. & n.m. & n.m. \\
Anoxic_1 & 0.2 & 0.2 & $<$ L.O.D. & n.m. \\
Anoxic_2 & 0.2 & n.m. & n.m. & n.m. \\
\hline
\end{tabular}

1 and 2 stand for replicates of each condition; n.m.: not measured; L.O.D: limit of detection. MeOH: methanol. 


\section{S4.4 Effect of alternating redox conditions on melamine biodegradation -}

\section{Results}
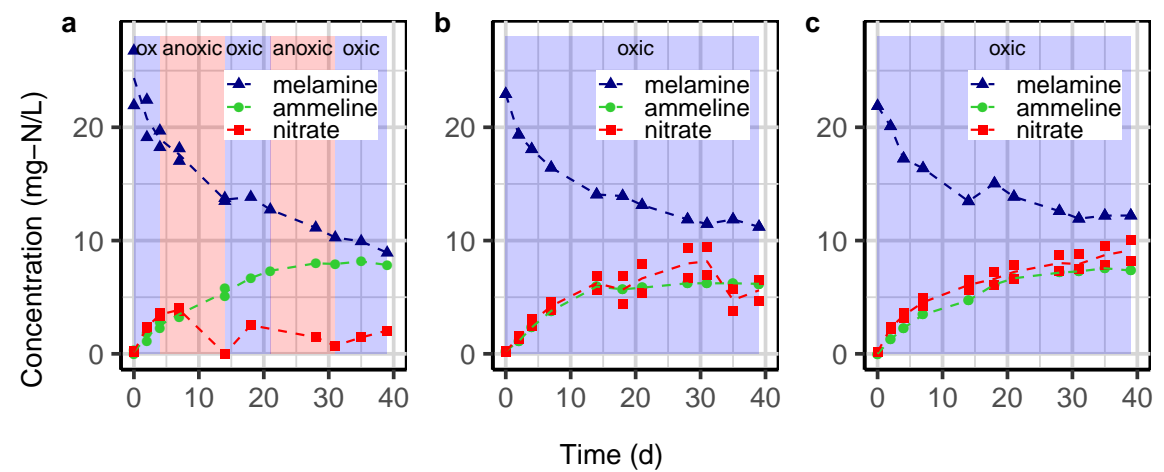

Figure S4.1: Melamine, ammeline and nitrate concentrations during melamine degradation. a: alternating oxic and anoxic conditions with methanol in anoxic period; b: oxic conditions with methanol; c: oxic conditions without methanol.

Table S4.5: Ammonium concentration (mg-N/L) in melamine degrading batches in alternating redox conditions.

\begin{tabular}{rrrrr}
\hline Condition & \multicolumn{5}{c}{ Day } \\
\hline 1 & 0 & 4 & 14 & 21 \\
\hline 2 & 1.22 & 0.17 & 0.05 & 2.32 \\
3 & 1.37 & 0.12 & 0.03 & 0.06 \\
4 & 1.37 & 0.12 & 0.02 & 0.97 \\
5 & 1.26 & 0.17 & 0.06 & 0.12 \\
& n.m. & n.m. & 0.03 & 0.07
\end{tabular}

1: methanol added in oxic and anoxic periods; 2: methanol added in anoxic period only; 3 : no methanol added in either period; 4: oxic with methanol; 5: oxic without methanol. n.m.: not measured. 


\section{S4.5 Melamine adsorption and desorption isotherm}

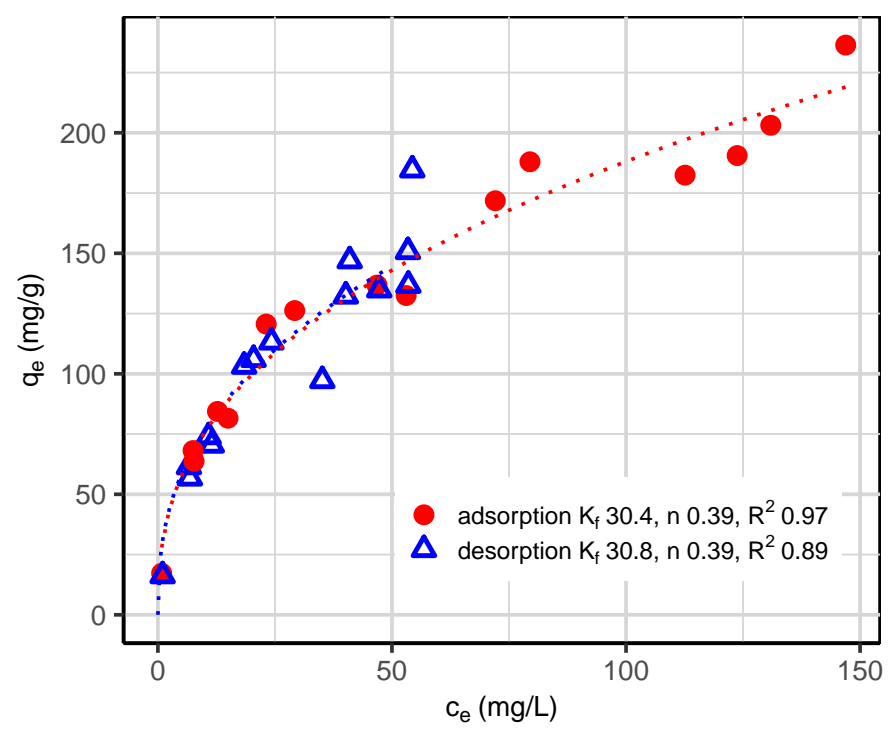

Figure S4.2: Adsorption and desorption isotherms and respective Freundlich parameters. 


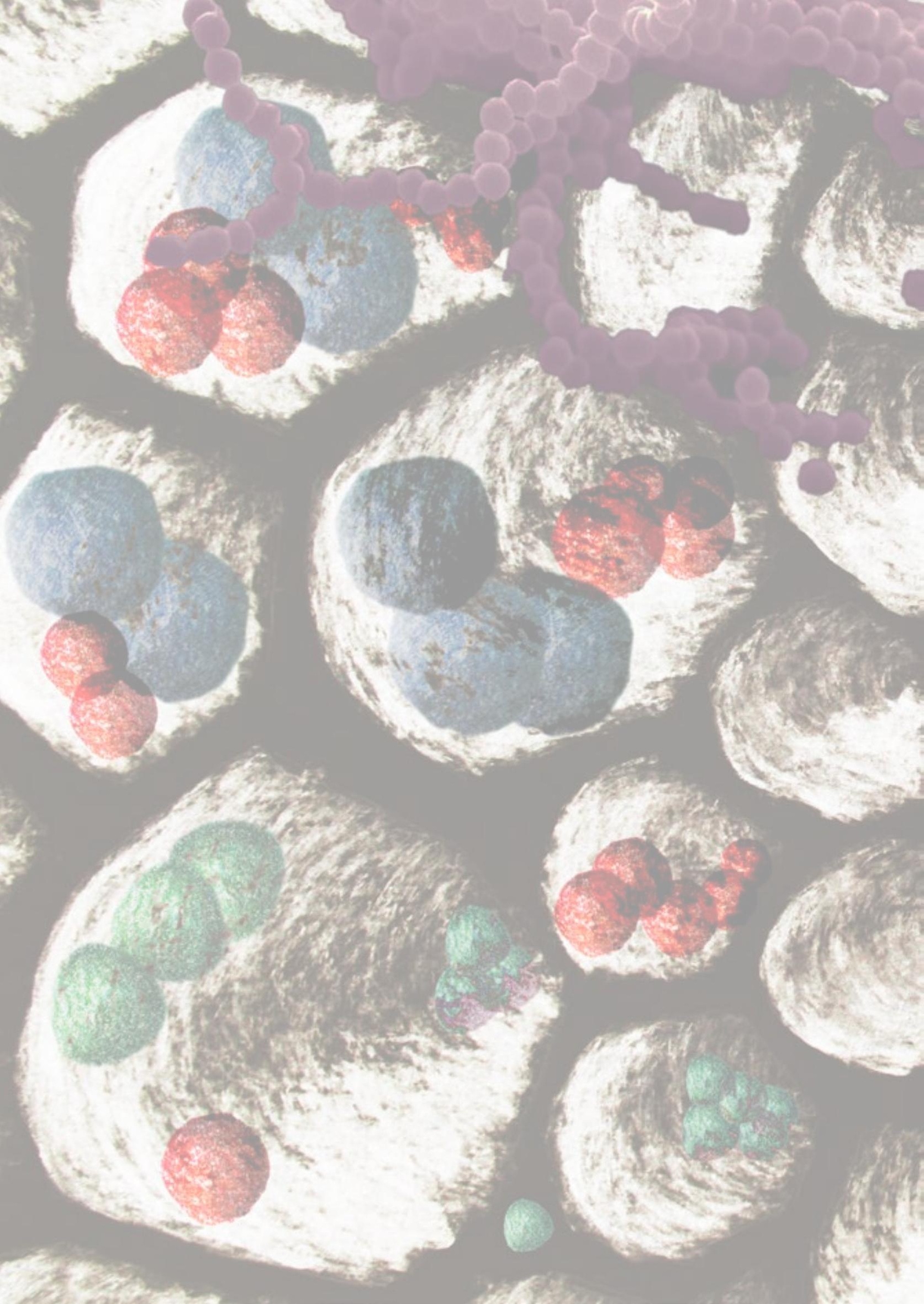



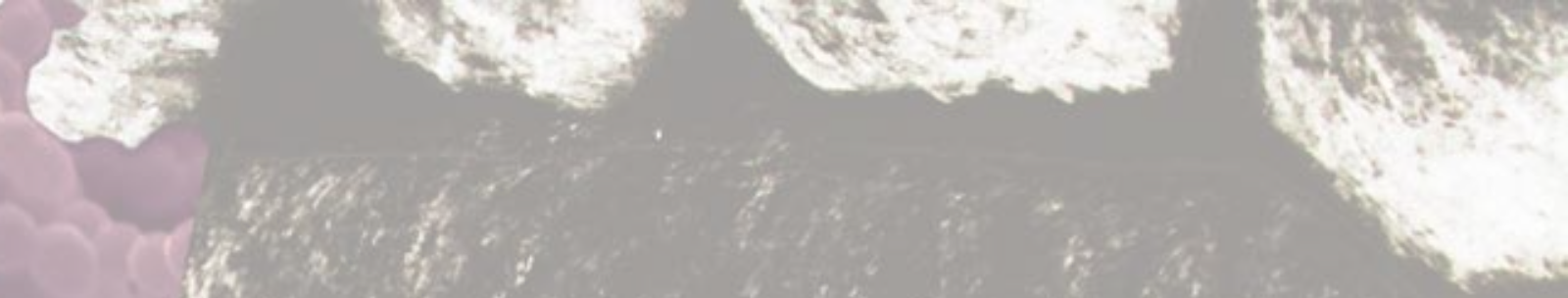

Chapter 5

\section{Biodegradation of melamine in activated carbon filters resulting in bioregeneration}

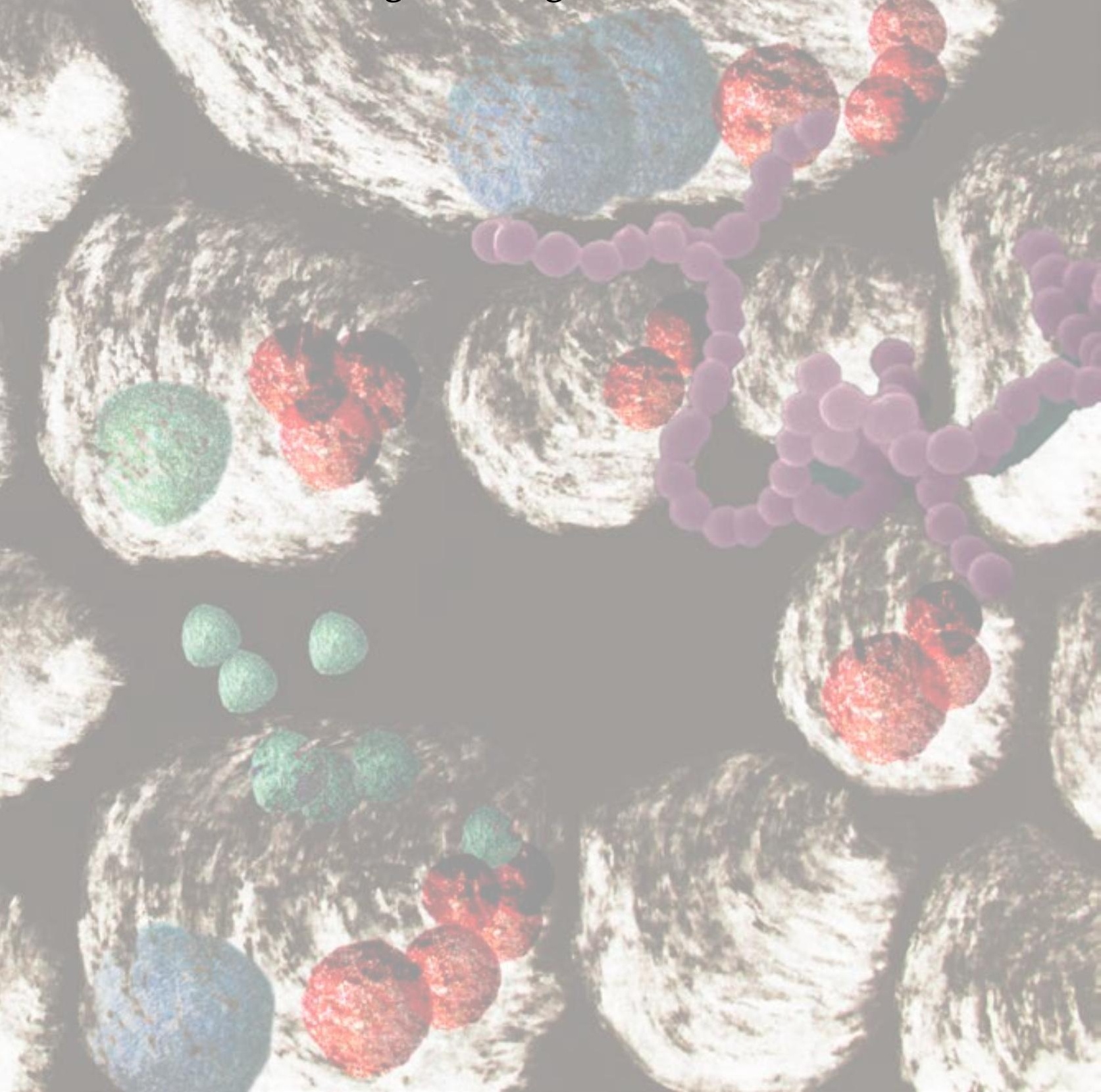




\section{ABSTRACT}

The contamination of surface water with micropollutants represents a challenge for the production of safe drinking water. Granular Activated Carbon (GAC) filters, used in drinking water production, can remove micropollutants through adsorption. GAC adsorption capacity reduces with time, hence GAC needs to be reactivated regularly, which is an energy intensive process that contributes significantly to the carbon footprint of water filtration with GAC. Micropollutants can also be removed in GAC filters through biodegradation when the conditions are suitable. This can provide more sustainable alternatives to GAC reactivation. The micropollutant melamine is frequently detected in surface water and is used in the present study as a model compound to quantify bioregeneration of GAC filters treating water contaminated with micropollutants. We assessed the contribution of melamine biodegradation to the total removal in addition to adsorption in lab-scale GAC filters inoculated with melamine degrading biomass. We evaluated the effect of an additional carbon source (methanol) and contact time on melamine removal efficiency using two different inoculation methods. We demonstrate that inoculation of GAC filters with melamine degrading biomass increases melamine removal efficiency by at least $29 \%$ in the absence of an additional carbon source. When an additional carbon source (methanol) is supplied to inoculated filters, melamine removal is almost complete (up to 99\%) and no breakthrough is observed. Finally, up to $98 \%$ bioregeneration can be obtained for a filter that is close to saturation after an additional carbon source (methanol) starts being supplied.

Manuscript in preparation:

Piai, L., Langenhoff, A., de Wilde, V., van der Wal, A. Biodegradation of melamine in activated carbon filters resulting in bioregeneration. 


\subsection{Introduction}

Surface water contains many persistent organic contaminants in the concentration range from ng/L to $\mu \mathrm{g} / \mathrm{L}$ (Ruff et al., 2015; Tröger et al., 2020), also referred to as micropollutants. Melamine is such a micropollutant, which is frequently detected in surface waters (RIWAMaas, 2017; RIWA-Rijn, 2018; Ruff et al., 2015; Seitz and Winzenbacher, 2017). This industrial chemical is used for a wide range of applications, including production of resins and plastics (Smit, 2018). Melamine can reach the surface water during the production process and/or during the use of products containing melamine and its derivatives (Smit, 2018).

Micropollutants, such as melamine, can be partly removed from surface water by applying Granular Activated Carbon (GAC) filters. GAC filters have been used for decades in drinking water production plants and their application for wastewater treatment is increasing (Benstoem et al., 2017; Reungoat et al., 2010). The removal efficiency of contaminants in GAC filters tends to reduce with time (Piai et al., 2020; Tröger et al., 2020), as contaminants accumulate on the GAC surface, exhausting its adsorption capacity. To recover the adsorption capacity, the GAC has to be reactivated frequently, which is typically done by applying high temperatures $\left(>700^{\circ} \mathrm{C}\right.$ ) (Mo et al., 2018; Worch, 2012). During reactivation, around $10 \%$ of GAC is lost (Bayer et al., 2005) and, therefore, replenishment with new GAC is needed. Life cycle analyses show that $80 \%$ of the carbon footprint of water filtration with GAC is related to producing or replacing the adsorbent (Mo et al., 2018). Hence, it is crucial to reduce the GAC reactivation frequency to reduce this carbon footprint.

One possible strategy to increase micropollutants removal efficiency in GAC filters and reduce the need for GAC reactivation is bioregenerating the GAC by biological degradation of the micropollutants. Bioregeneration is achieved when micropollutants are removed by biodegradation from the liquid phase, causing previously adsorbed micropollutants to desorb from the GAC, due to a gradient in the concentration from the GAC to the liquid. This process can continue as long as desorption is not hindered and biodegradation can take place (Aktaş and Çeçen, 2007; Sirotkin et al., 2001).

Biodegradation of micropollutants in GAC filters requires the presence of microorganisms capable of degrading the contaminants under the filter conditions. It has been shown that melamine is not sufficiently removed in pilot-scale GAC filters and is not biodegraded by indigenous biomass grown in GAC filters used for drinking water production (Brunner et al., 2020; Piai et al., 2020a). Yet, melamine can be biodegraded by specific microorganisms 
isolated from soil and industrial wastewater treatment (El-Sayed et al., 2006; Takagi et al., 2012; Wang et al., 2014). We previously demonstrated that melamine degrading microorganisms can be used to restore $28 \%$ of the GAC adsorption capacity through bioregeneration (Piai et al., 2020b).

In this paper, we describe the removal of melamine in lab-scale GAC filters that are inoculated with melamine degrading biomass, thus turning into biological activated carbon (BAC) filters. We assessed the effect of an additional carbon source (methanol) and empty bed contact time (EBCT) on melamine removal efficiency, as well as the efficacy of two different inoculation methods. Moreover, we studied melamine desorption from the filter bed by feeding the filter with influent without melamine. Finally, we were able to quantify GAC bioregeneration in a nearly saturated filter when melamine biodegradation rates increased.

\subsection{Materials and methods}

\subsubsection{Experimental setup}

Melamine removal was tested in lab-scale filters with and without inoculation of melamine degrading biomass. The filters were made of glass cylinders with $2.6 \mathrm{~cm}$ internal diameter and $20 \mathrm{~cm}$ length. Each filter had 2 sampling points located at $6.0-8.0 \mathrm{~cm}(\mathrm{~A})$ and $2.5-4.5$ $\mathrm{cm}$ (B) from the bed bottom (Figure 5.1). The dead volume of the sampling points was filled with clean glass wool before each experiment. Before being used in the filters, the GAC was boiled in demineralized water for 10 minutes to remove entrapped air. Once inside the filters and before starting experiments, GAC was flushed with tap water until the effluent $\mathrm{pH}$ stabilized $(\mathrm{pH} \sim 8)$.

Nylon meshes at the top and bottom of the filters prevented GAC wash out. Initial bed height was $10 \mathrm{~cm}$, and changed to $12-16 \mathrm{~cm}$ at the end of the experiments due to biomass growth and bed expansion. The remaining volume of the column was permanently filled with influent. Despite the variation in bed height, a fixed bed volume (BV) of $0.053 \mathrm{~L}$ was used for calculations, corresponding to the BV in the beginning of the experiments. This fixed BV was used since the GAC mass in the filter bed did not change during the experiment and the increased volume in the filter bed corresponded either to void space or biomass volume, not accounting as adsorbent volume. 


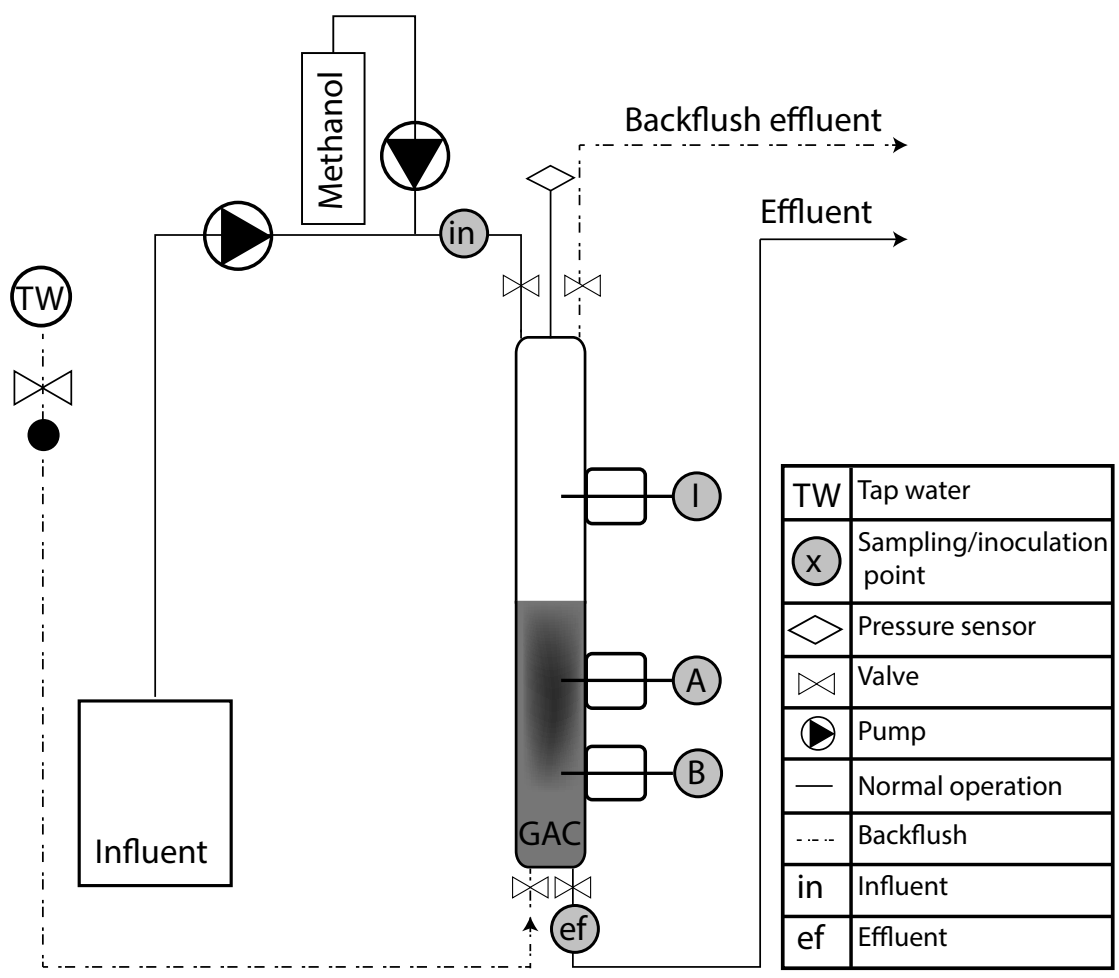

Figure 5.1: Schematic representation of experimental set-up. Column dimensions and sampling points positions are to scale.

Influent consisted of a nitrogen-free nutrient solution spiked with melamine $(8.0 \pm 0.8 \mathrm{mg} / \mathrm{L})$. The detailed influent composition is described in the Supplementary Information (Table S5.1). Influent was pumped through the filters in a downwards direction by a peristaltic pump (Ismatec Reglo ICC). Actual flow was checked periodically by weighing the effluent of each filter at a determined time interval. The flow rate was adjusted when needed to obtain a desired EBCT. When methanol was used in the experiment, a solution of $0.1 \%$ methanol stored at $10^{\circ} \mathrm{C}$ was mixed with the influent by a second peristaltic pump. The flow of influent:methanol was approximately 100:1.

The pressure at the top of the filters was monitored using DMP 331Pi pressure sensors (BD sensors). If overpressure exceeded 200 mbar, automatic backflushing was initiated. In addition, regular backflush with tap water was performed weekly for 5 minutes at a flow rate of $3-6 \mathrm{~L} / \mathrm{h}$. Backflush flow was controlled using a flow controller (ES-FLOW' Bronkhorst $\left.{ }^{\circledR}\right)$. Temperature in the filters was monitored with Teflon ${ }^{\circledR}$ coated temperature 
sensors (ProSense Pt-100) and remained between 27 and $30^{\circ} \mathrm{C}$. LabVIEW 2018 was used to monitor filter pressure, effluent temperature and $\mathrm{pH}$ and to control valves for backflush.

\subsubsection{Melamine removal}

Melamine removal due to adsorption or adsorption combined with biodegradation was assessed in 6 experiments. We tested the effect of an additional carbon source (methanol), the inoculation of melamine degrading biomass and EBCT on melamine removal in labscale GAC filters (Table 5.1). BAC 1 - 5 were inoculated with activated sludge containing melamine degrading biomass, which originated from a wastewater treatment plant (Azomures, Romania) treating industrial wastewater containing melamine (Piai et al., 2020b). A non-inoculated filter was used as control for assessing melamine removal due to adsorption only.

Table 5.1: Melamine removal experiments performed in lab-scale GAC filters.

\begin{tabular}{rrrrrr}
\hline Filter & $\begin{array}{r}\text { EBCT } \\
(\mathrm{min})\end{array}$ & $\begin{array}{r}\text { Filter } \\
\text { inoculation }\end{array}$ & $\begin{array}{r}\text { Methanol in } \\
\text { influent }\end{array}$ & $\begin{array}{r}\text { Duration } \\
(\mathrm{BVs})\end{array}$ & $\begin{array}{r}\text { Desorption } \\
(\mathrm{BVs})\end{array}$ \\
\hline GAC 1 & $18 \pm 2$ & No & No & 6,067 & NA \\
BAC 1 & $32 \pm 3$ & Direct & No/Yes & 14,000 & $>8,300$ \\
BAC 2 & $32 \pm 3$ & Direct & Yes & 4,923 & NA \\
BAC 3 & $32 \pm 3$ & Indirect & Yes & 5,829 & NA \\
BAC 4 & $18 \pm 2$ & Direct & Yes & 8,724 & $>6,000$ \\
BAC 5 & $18 \pm 2$ & Indirect & Yes & 6,990 & NA \\
\hline
\end{tabular}

NA: not applicable.

Melamine desorption was assessed in BAC 1 and 4 by feeding the filters with influent without melamine after approximately 8,300 and 6,000 BVs respectively. During this stage, the filters were no longer backflushed.

Experiment BAC 1 consisted of 3 phases. In phase I, the influent did not contain methanol. In phase II, which started after approximately 6,200 BVs, methanol was added to the influent in the same way as in BAC 1-4. In phase III, which started after approximately $8,300 \mathrm{BVs}$, the filter was fed with influent with methanol, but without melamine. 
Specific melamine removal ( $r_{s}$, in mg melamine/g GAC) was calculated based on the amount of melamine $(m$, in $\mathrm{mg})$ removed in the filter in a specific time interval, the bed volume $(B V$, in $\mathrm{L}$ ) and bed density ( $\rho_{b}$, in $\left.\mathrm{g} / \mathrm{L}\right)$, according to equation 5.1 :

$$
r_{s}=\frac{m}{B V \rho_{b}}
$$

The value of $m$ was obtained from the area between the influent and effluent concentration curves using the spline interpolation method (example in Figure S5.1). The value of bed density used was $450 \mathrm{~g} / \mathrm{L}$, based on the material fact sheet from the GAC supplier.

\subsubsection{Inoculation of BAC filters}

\section{Direct}

In experiments BAC 1, 2 and 4, filters were directly inoculated with melamine degrading biomass on days 0,7 and 14 . A volume of $1.6 \mathrm{~mL}$ sludge was inoculated at points I and A (Figure 5.1). In experiment BAC 1, the filter was reinoculated 5 times between days 81 and $103(3,500-4,500 \mathrm{BVs})$.

\section{Indirect}

In experiments BAC 3 and 5, the GAC was inoculated with melamine degrading biomass in batches, before being used in the filters. The batches contained $7.5 \mathrm{~g}$ fresh GAC, $10 \mathrm{~mL}$ melamine degrading biomass, $3 \mathrm{~g} / \mathrm{L}$ methanol and mineral media (composition in Table S5.1) containing $1000 \mathrm{mg} / \mathrm{L}$ melamine in a total liquid volume of $550 \mathrm{~mL}$. After 21 days, the liquid medium was removed from each batch, the BAC was washed 3 times with fresh mineral media to remove suspended biomass, after which the BAC was stored wet at $4^{\circ} \mathrm{C}$ for $48 \mathrm{~h}$. After this period, the BAC was transferred to the filter containing fresh GAC, in a ratio of 1:3 (BAC:GAC). The BAC and GAC were mixed by inverting the filter several times. The filters were stored at $4^{\circ} \mathrm{C}$ for approximately $16 \mathrm{~h}$, after which they were installed and the experiments started.

\subsubsection{BAC bioregeneration}

Bioregeneration was calculated for BAC 1, according to the following steps:

1) BAC load (phase I): BAC load at the end of phase I ( $q_{I}$, in mg melamine/g BAC) was calculated, assuming equilibrium between the filter bed and influent concentration at the end of that phase. The value of $q_{I}$ was calculated using the average influent concentration 
( $c_{0}$ in $\left.\mathrm{mg} / \mathrm{L}\right)$ during phase I and the Freundlich isotherm parameters $\left(K_{f}\right.$ in $(\mathrm{mg} / \mathrm{g}) /(\mathrm{mg} / \mathrm{L})$ and $n$ (dimensionless)) obtained in previous studies (Piai et al., 2020b) and corrected for the temperature of the filters.

2) Desorbed melamine (phases II and III): The amount of melamine desorbed ( mel $_{\text {des }}$, in $\mathrm{mg}$ melamine/g BAC) from BAC 1 in phases II and III was calculated from the amount of nitrogen desorbed $\left(N_{\text {des }}\right.$, in $\mathrm{mg} \mathrm{N} / \mathrm{g}$ BAC), assuming melamine full mineralization. The value of $N_{\text {des }}$ was obtained from the area between the effluent and influent total nitrogen concentration curves. Total nitrogen corresponded to the sum of the nitrogen present in ammonium and melamine.

3) Bioregeneration efficiency: Bioregeneration at different intervals of treated volume was quantified by dividing the cumulative amount of desorbed melamine by the GAC load in the end of phase I.

\subsubsection{Modelling melamine adsorption in fixed-bed filters}

Melamine adsorption in fixed-bed filters was modelled using the Homogeneous Surface Diffusion Model (HSDM) (Worch, 2012) implemented in MATLAB $^{\circledR}$. The equilibrium condition was represented by the Freundlich isotherm. The values used for each model parameter are presented in Table S5.2. Surface and film diffusion coefficients were based on previous fitting of batch kinetic experiments and breakthrough curves obtained in the same setup for a comparable micropollutant (pyrazole) (Piai et al., manuscript in preparation). Experimental data from the non-inoculated filter (GAC 1) was fitted to the HSDM to validate the model output.

\subsubsection{Analysis}

Samples for melamine and methanol analysis were filtered with $0.2 \mu \mathrm{M}$ polyethersulfone membrane filters and stored at $-20^{\circ} \mathrm{C}$ until analysis. Samples for methanol analysis were spiked with $10 \%(\mathrm{v} / \mathrm{v})$ of $4 \mathrm{M}$ formic acid before being stored.

Dissolved oxygen concentrations were measured using non-invasive oxygen sensors (Spot SP-PSt3, PreSens) and an oxygen meter (Fibox 4). Ammonium was measured using Hach Lange colorimetric kits (LCK 303 and LCK 305) and a spectrophotometer (Hach Lange DR 3900). Methanol was measured with gas chromatography as described in Jourdin et al. (2018). Concentrations of melamine and its transformation product ammeline were measured by liquid chromatography coupled to UV-detection, using a Luna CN analytical 
column (Phenomenex ${ }^{\circledR}$ ) and an isocratic flow of $1 \mathrm{~mL} / \mathrm{min}$ phosphate buffer $(5 \mathrm{mM}, \mathrm{pH}$ 6.7), as described in Piai et al. (2020b). In the same system, the other transformation products ammelide and cyanuric acid were detected, but could not be quantified due to incomplete peak separation.

\subsection{Results and discussion}

\subsubsection{Melamine adsorption - modelled and experimental breakthrough curves}

The experimental breakthrough curve of the non-inoculated filter (GAC 1) was fitted to the adsorption model (HSDM) (Figure 5.2). First, the experimental breakthrough was compared to the model output, using the Freundlich coefficients as calculated in Piai et al. (2020b). We observed that the model predicted a later breakthrough than obtained experimentally. The earlier breakthrough observed in our experiment is likely related to the higher temperature in which the filters were operated $\left(27-30^{\circ} \mathrm{C}\right)$, compared to the temperature at which the adsorption isotherms was performed $\left(20^{\circ} \mathrm{C}\right)$. Previous experiments have shown that melamine adsorption decreases with increasing temperatures (Piai et al., 2020a). Therefore, the experimental breakthrough was fit to several model outputs assuming different degrees of reduced adsorption due to the higher temperature. We observed that the best fitting was produced when $10 \%$ less adsorption was taken into account (Figure 5.2). The alternative Freundlich coefficients calculated for this scenario $\left(K_{f}=26 \mathrm{~L} / \mathrm{g}\right)$ and $\left.n=0.39\right)$ were then used as input for all modelled breakthrough curves presented in this study. We also evaluated the model output using the Langmuir equation to represent the equilibrium condition. Even though the Langmuir equation fits the isotherm data well $\left(\mathrm{R}^{2}=0.94\right)$, its use in the HSDM underestimates the adsorption capacity in the filter, and therefore is not used in this study. 


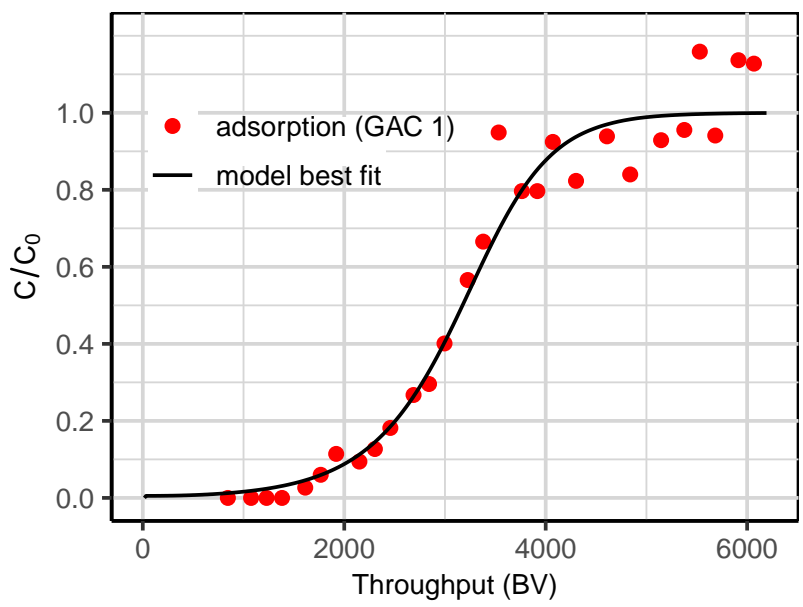

Figure 5.2: Experimental breakthrough curve of non-inoculated filter and fitted curve of homogeneous surface diffusion model (HSDM).

\subsubsection{Melamine adsorption and biodegradation}

We first studied an inoculated filter without addition of methanol as a carbon source. We compared the breakthrough curve of our inoculated filter (BAC 1) with the modelled breakthrough curve for adsorption only (Figure 5.3). For the inoculated filter, melamine breakthrough occurred at a higher throughput, compared to the modelled breakthrough curve.

Melamine specific removal $\left(r_{s}\right)$ was calculated for the modelled results until $100 \%$ breakthrough and for the inoculated filter until 6,000 BV, before initial experimental conditions were changed. We observed that the $r_{s}$ of the inoculated filter $(80 \mathrm{mg} / \mathrm{g})$ was $29 \%$ higher than the removal of the fitted curve with adsorption only ( $57 \mathrm{mg} / \mathrm{g}$ ) (Figure 5.3). The fact that we observed a higher melamine removal in the inoculated filter demonstrates that melamine biodegradation is a significant additional removal mechanism. 


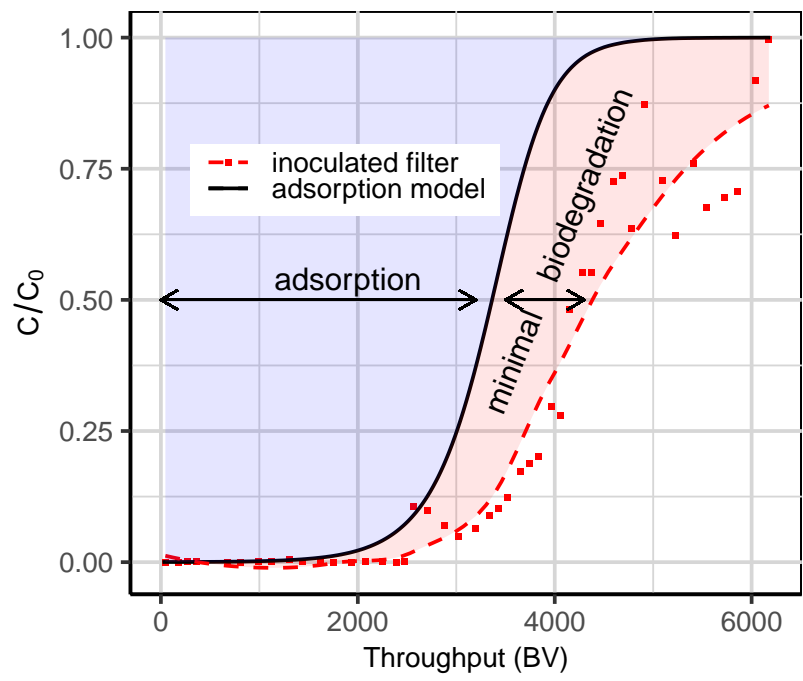

Figure 5.3: Relative melamine concentration in effluent of an inoculated filter without methanol (BAC 1) and modelled breakthrough curve. Dotted line represents regression line fitted with the loess method.

Reinoculating the filter between 3,500 and 4,500 BVs had only a temporary and local effect on melamine biodegradation, as indicated by the ammonium concentrations in the different filter positions for these BVs (Figure S5.2). Ammonium is produced during melamine biodegradation and accumulates under anoxic conditions (Piai et al., 2020b), which was the prevailing redox condition in this filter. An increase in ammonium concentration was measured temporarily at the top of the filter (position A), where biomass was introduced during reinoculation of the filter (Figure S5.2). However, this effect was not observed further down the filter (position B) nor in the effluent, and ammonium concentrations decreased soon after reinoculation stopped $(>4,500 \mathrm{BVs})$. These results indicate that filter reinoculation did not significantly contribute to an increased melamine removal.

\subsubsection{Melamine adsorption and biodegradation with methanol as additional carbon source}

Figure 5.4 shows complete melamine removal for inoculated BAC in the presence of methanol as an additional carbon source (both direct and indirect inoculation). For comparison, the modelled breakthrough curve for adsorption only is also shown (Figure 5.4). Effluent breakthrough did not happen in the inoculated filters with methanol within the time-frame of the experiments $(6,000 \mathrm{BVs})$, whereas $100 \%$ breakthrough is predicted for the same period by the adsorption model. These results clearly show that 
melamine biodegradation is improved in the presence of methanol, which contributes to extending the BAC filter life-time.

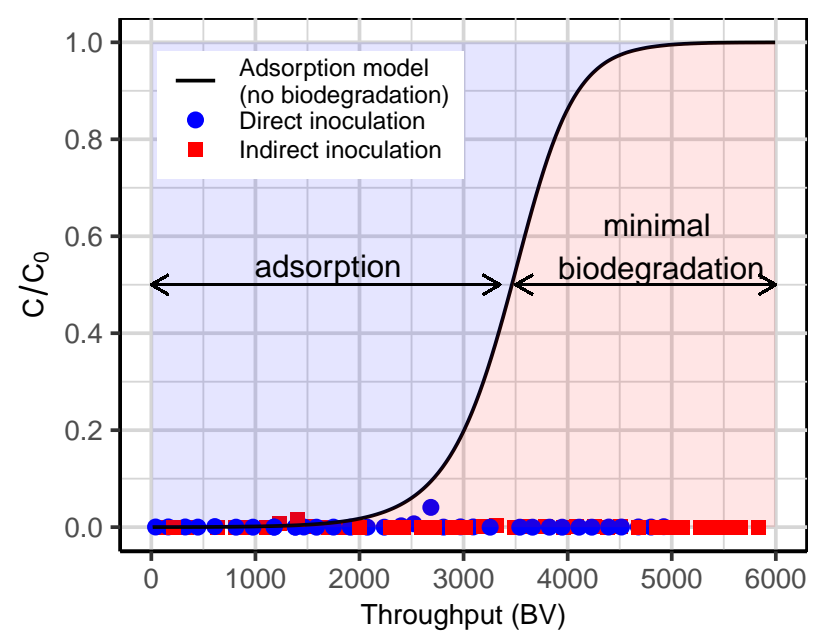

Figure 5.4: Relative melamine concentration in effluent of directly and indirectly inoculated filters receiving methanol, operated at $32 \mathrm{~min}$ EBCT (BAC 2 and 3).

The comparison between inoculated filters with (Figure 5.4) and without (Figure 5.3) methanol shows that an additional carbon source (methanol) is essential to avoid melamine breakthrough. The positive effect of an additional carbon source on biodegradation of melamine and its transformation products has been previously demonstrated in batch and column experiments (Galíndez-Nájera et al., 2009; Piai et al., 2020b; Shelton et al., 1997; Takagi et al., 2012). Methanol is needed to compensate for the low C:N ratio of melamine $(0.5 \mathrm{~mol} \mathrm{C} / \mathrm{mol} \mathrm{N})$. The $\mathrm{C}: \mathrm{N}$ ratio in our filters influent was 0.6 - 0.7 (molar basis). It has been shown that a C:N ratio of 1 or higher is required for complete biodegradation of melamine and its transformation products (Galíndez-Nájera et al., 2009; Takagi et al., 2012). Our experiments show that $\mathrm{C}: \mathrm{N}$ ratios lower than those previously recommended can still maintain high melamine removal efficiency in BAC filters. Average methanol concentration in the filters influent ranged between 1.4 and $2.9 \mathrm{mg} / \mathrm{L}$ depending on the filter. In the filter bed (positions A and B), the methanol concentration was always below the limit of detection $(1 \mathrm{mg} / \mathrm{L})$. Even though methanol was already biodegraded at the top of the filter bed, its presence in the influent was enough to stimulate melamine biodegradation throughout the filter and avoid melamine breakthrough from the filters. 
We investigated if melamine could also be removed efficiently at shorter contact times (EBCT of $18 \mathrm{~min}$ instead of $32 \mathrm{~min}$ ). We observed that melamine removal in those filters was comparable to the removal in the filters with longer contact times (Figure 5.5). Only the upper layers of the filter bed (position A) showed higher melamine concentrations (Figure 5.5). Further down the column (position B), melamine concentrations were higher than in filters operated at longer contact times (EBCT $32 \mathrm{~min}$ ), but still below $5 \%$ of the influent concentration. Position B of filters operated at shorter contact times corresponds to an EBCT of 10-15 min. Melamine concentrations in position B indicate that an EBCT of around 15 min is the minimum time required to maintain more than $95 \%$ melamine removal. Moreover, regardless of the inoculation method, $19 \mathrm{~min}$ is enough to obtain more than $99 \%$ melamine removal, as long as an additional carbon source is supplied. EBCTs between 18 and $32 \mathrm{~min}$ are within the practical values applied in full-scale GAC filters e.g. in drinking water production plants (Kennedy et al., 2015).
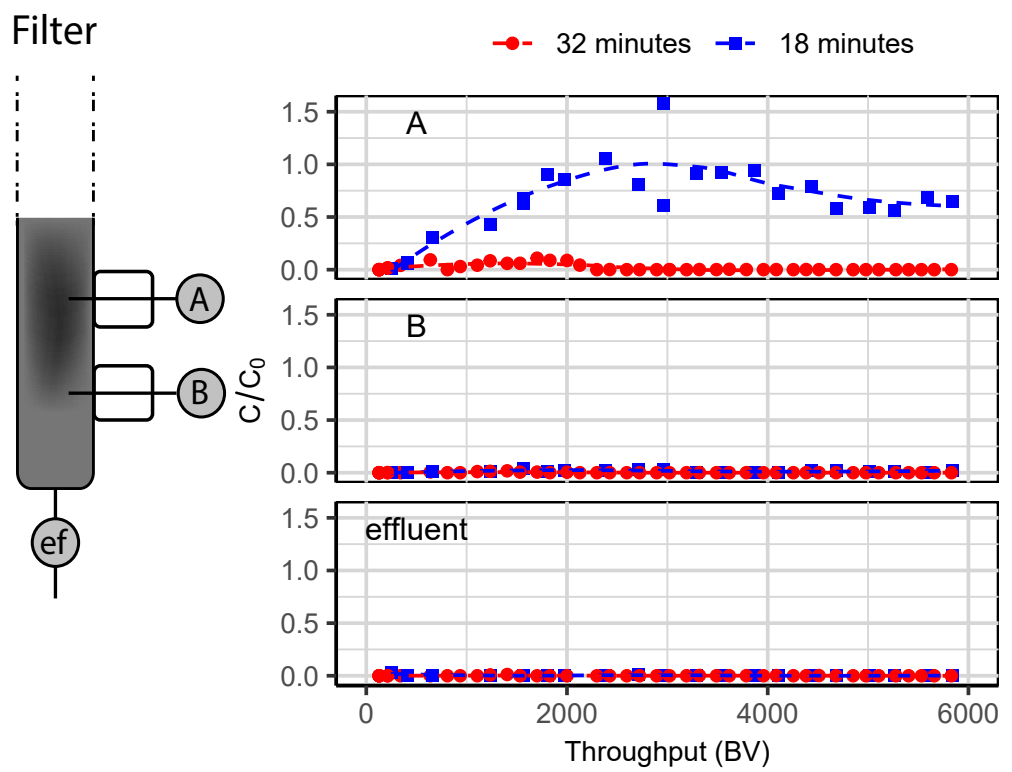

Figure 5.5: Relative melamine concentration in intermediate positions (A and B) and effluent of indirectly inoculated filters with methanol. Dotted lines represent regression line fitted with the loess method.

\section{Melamine desorption}

A decrease in influent concentration can cause desorption of previously adsorbed melamine, as melamine adsorption is fully reversible (Piai et al., 2020b). We assessed melamine 
desorption in the directly inoculated filter in the presence of methanol (EBCT $18 \mathrm{~min}$, BAC 4) by feeding the filter with influent without melamine after 6,000 BVs. During this (desorption) phase, the melamine concentration gradually decreased in positions A and B and increased temporally in the effluent, followed by a rapid decrease (Figure 5.6). Nevertheless, the absolute melamine concentration in the effluent remained rather low $(<0.064 \mathrm{mg} / \mathrm{L})$. Interestingly, higher melamine concentrations were detected in lower layers of the filter (position B) compared to the upper layers (position A). Possibly, melamine biodegradation rates are higher in the upper layers of the filter. A vertical gradient of biomass activity in fixed-bed filters, with higher activity closer to the influent position, has also been reported previously (Chen et al., 2016; Gibert et al., 2013). 


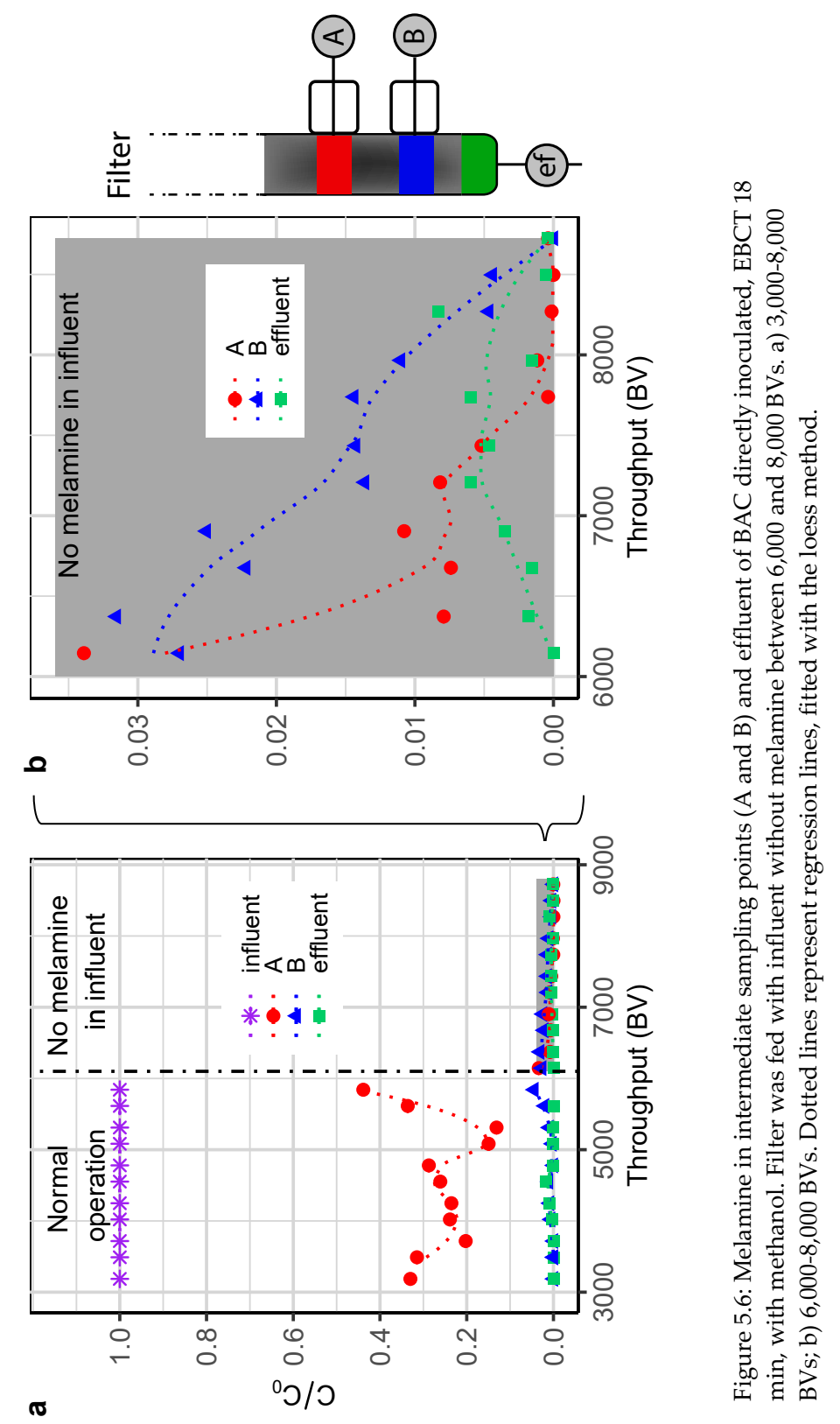




\subsubsection{BAC bioregeneration}

We divided the operation of the directly inoculated filter (BAC 1) into three phases: i) phase I, influent without methanol, ii) phase II ( $>6,200 \mathrm{BVs})$, influent with methanol and iii) phase III (>8,300 BVs), influent without melamine and with methanol.

We observed that melamine biodegradation rate increased significantly in phase II after methanol was added to the influent. The increased biodegradation rate is evident from the decreasing melamine concentrations in the column at positions A and B as well as in the effluent. We also observed an concomitant increase in ammonium concentrations in phase II (Figure 5.7). In phase III, when the filter did not receive melamine, melamine and ammonium were still detected in the columns at positions A, B and the effluent (Figure 5.7), indicating that melamine desorbed from the BAC and was partially biodegraded during this third phase.

When both melamine and methanol were present in the influent (phase II), the total nitrogen concentration in the effluent (as melamine- $\mathrm{N}$ and ammonium- $\mathrm{N}$ ) exceeded the influent concentration (Figure 5.8). This excess nitrogen must originate from biodegradation of desorbed melamine and/or its transformation products (ammeline, ammelide and cyanuric acid) (Cook and Huetter, 1981). Desorption of melamine and/or its intermediate transformation products from the BAC is the result of a concentration gradient from the $\mathrm{BAC}$ to the liquid, as a consequence of melamine biodegradation in the liquid phase (phase II). 

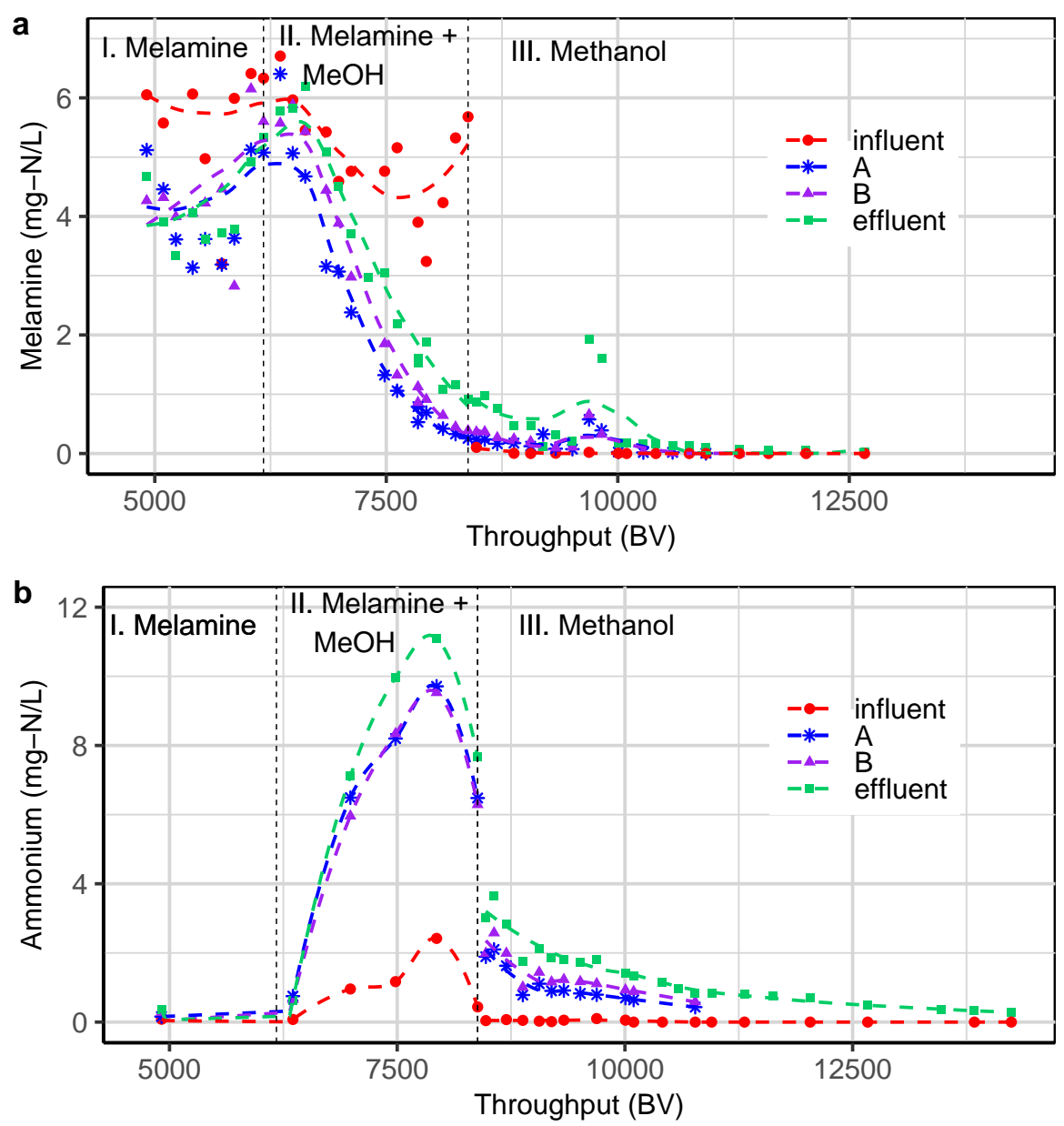

Figure 5.7: Melamine (a) and ammonium (b) concentration (in $\mathrm{mg}-\mathrm{N} / \mathrm{L}$ ) in influent, intermediate positions (A and B) and effluent of BAC directly inoculated, EBCT $32 \mathrm{~min}$ before and after methanol addition to the influent. Dotted lines represent regression line fitted with the loess method. 


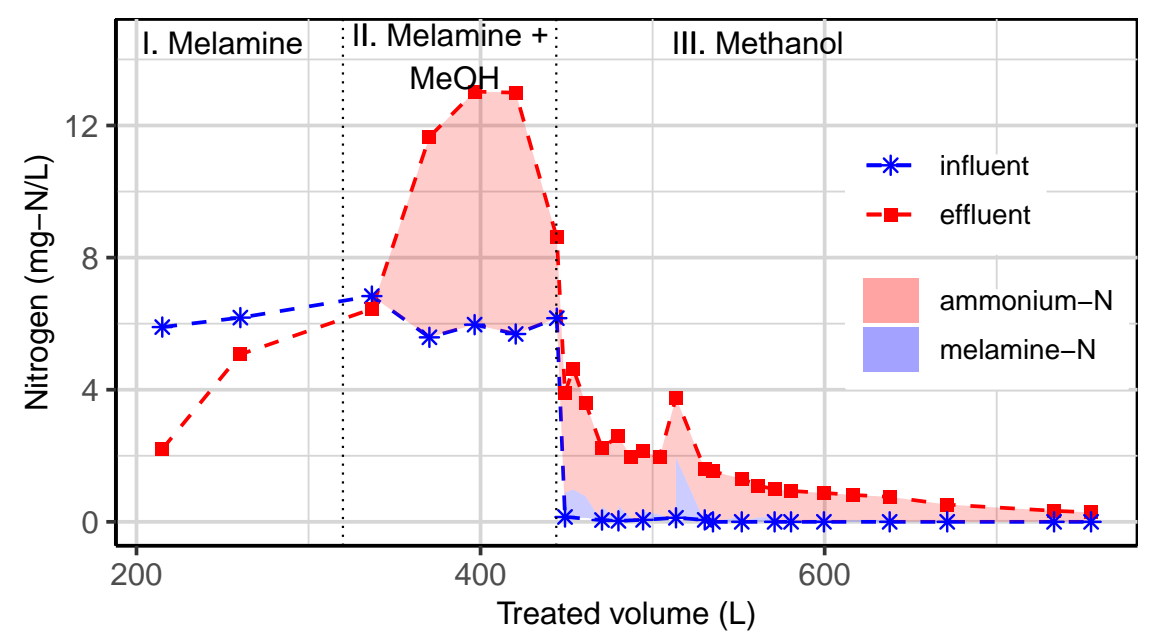

Figure 5.8: Total nitrogen concentration (from melamine and ammonium) in influent and effluent of BAC directly inoculated. Coloured area corresponds to the amount of $\mathrm{N}$ originating from melamine desorption, measured as melamine (blue) or ammonium (red).

Interestingly, not all desorbed melamine during phase III was biodegraded, as both melamine and ammonium could be measured in the effluent (Figure 5.8). The nonbiodegraded melamine in the effluent most likely originated from the lower layers of the filter bed, where biomass activity is likely lower (Gibert et al., 2013). This is in agreement with the consistently higher melamine concentrations in the effluent compared to in the column (positions A and B) once desorption occurs (phases II and III, Figure 5.7a).

\subsubsection{Quantification of bioregeneration}

Bioregeneration efficiency was quantified based on the nitrogen mass balance in the filter, with the following assumptions: 1) Nitrogen uptake due to biomass growth is neglectable, given that biomass growth in the filter is limited by the low methanol concentration $(<3$ $\mathrm{mg} / \mathrm{L}$, C:N ratio $0.5-0.6 \mathrm{~mol} \mathrm{C} / \mathrm{mol} \mathrm{N}$ in phase II); 2) Biodegraded melamine is fully mineralized and no transformation products (ammeline, ammelide, cyanuric acid) accumulate neither in the liquid nor on the GAC phase. This can be assumed, as ammeline was measured only at low concentrations $(<0.6 \mathrm{mg} / \mathrm{L})$ in all samples of this filter, and neither ammelide nor cyanuric acid were detected throughout the whole filter operation. Additionally, previous experiments with melamine biodegradation using the same inoculum showed no accumulation of melamine transformation products at anoxic conditions (Piai et al., 2020b). We therefore expect limited contribution of melamine transformation products to the nitrogen mass balance. 
The BAC load $\left(q_{I}\right)$ before methanol addition to the filter (end of phase I) was calculated assuming equilibrium between the influent concentration and the activated carbon. It is likely that the actual $q_{I}$ is lower than the calculated load using this approach, given that melamine effluent concentration was only $80 \%$ of the influent concentration. However, it is not possible to assess the actual $q_{I}$, as both melamine adsorption and biodegradation occurred in parallel. Given that bioregeneration is calculated based on the amount of melamine desorbed, divided by the BAC load in phase I, an overestimation of $q_{I}$ would result in an underestimation of the bioregeneration efficiency. Therefore, we consider that the bioregeneration calculated using this approach corresponds to the lowest achievable bioregeneration efficiency.

Bioregeneration efficiency is depicted in Figure 5.9. After 7,000 BVs, approximately $98 \%$ of the melamine adsorbed to the BAC in phase I desorbed, and most of the desorbed melamine $(81 \%)$ was biodegraded. Bioregeneration efficiencies in fixed-bed filters ranging from 2095\% for GAC loaded with 2-nitrophenol, surfactants, benzene and toluene have been reported in literature (Klimenko et al., 2003; Putz et al., 2005; Smolin et al., 2020). Our results provide additional evidence that high levels of bioregeneration can be obtained when favourable conditions for biodegradation are provided.

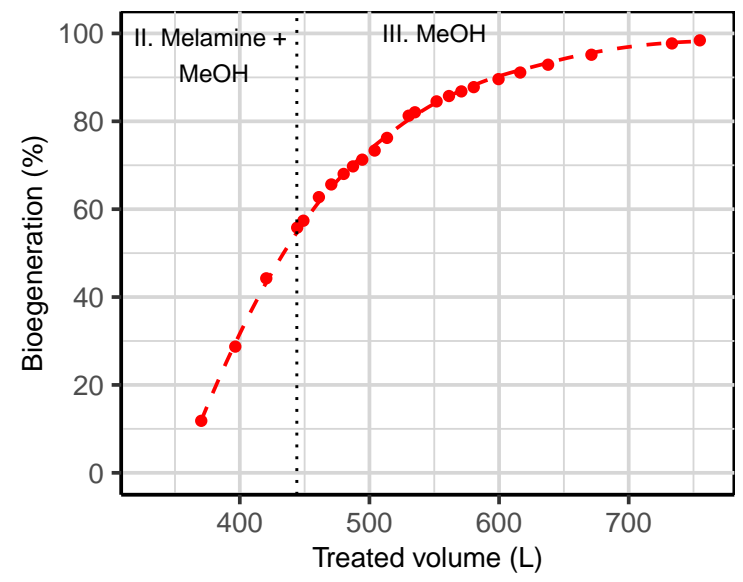

Figure 5.9: Bioregeneration efficiency of BAC 1 after addition of methanol.

\subsection{Conclusions}

- Melamine biodegradation in BAC filters results in higher melamine removal and an extension of filter life-time compared to GAC filters, where adsorption is the only removal process. 
- Melamine biodegradation was stimulated by adding melamine degrading biomass.

- Melamine biodegradation rates increased by adding a carbon source (methanol) to the filter, thus regenerating a nearly saturated filter bed.

- Investigating the efficiency of this process at lower micropollutant concentrations with a variety of micropollutants will help identify opportunities for applying bioregeneration to GAC filters used in water treatment and reduce the need for GAC thermal reactivation.

\section{Acknowledgements}

This work was financially supported by Evides Water Company N.V. (Rotterdam, The Netherlands). We thank Nijhuis Water Technology B.V. (Doetinchem, The Netherlands) for providing the melamine degrading sludge used in the experiments and Nadine Boelee of Nijhuis Water Technology B.V for discussing the experimental setups and results. We also thank Chufeng Gong and Yuchen Qian for executing part of the experiments and Jouke Dykstra and Mingyi Jia for creating the code for the adsorption model. 


\section{Supplementary information to Chapter 5}

Table S5.1: Composition of filters influent and batch media.

Concentration $(\mathrm{g} / \mathrm{L})$

Filter influent Batch media

\begin{tabular}{|c|c|c|}
\hline Buffer & & \\
\hline $\mathrm{Na}_{2} \mathrm{HPO}_{4} .2 \mathrm{H}_{2} \mathrm{O}$ & 0.018 & 1.78 \\
\hline $\mathrm{KH}_{2} \mathrm{PO}_{4}$ & 0.09 & 1.36 \\
\hline \multicolumn{3}{|l|}{ Macro nutrients } \\
\hline $\mathrm{CaCl}_{2} .2 \mathrm{H}_{2} \mathrm{O}$ & 0.003 & $4.8 * 10^{-2}$ \\
\hline $\mathrm{MgSO}_{4} .7 \mathrm{H}_{2} \mathrm{O}$ & 0.008 & $5.4 * 10^{-2}$ \\
\hline \multicolumn{3}{|l|}{ Trace elements } \\
\hline $\mathrm{FeCl}_{2} .4 \mathrm{H}_{2} \mathrm{O}$ & $2.5 * 10^{-4}$ & $1.2 * 10^{-3}$ \\
\hline $\mathrm{CoCl}_{2} \cdot 6 \mathrm{H}_{2} \mathrm{O}$ & $2.5 * 10^{-4}$ & $1.2 * 10^{-3}$ \\
\hline $\mathrm{MnCl}_{2} .4 \mathrm{H}_{2} \mathrm{O}$ & $6.25 * 10^{-5}$ & $3 * 10^{-4}$ \\
\hline $\mathrm{CuCl}_{2} .2 \mathrm{H}_{2} \mathrm{O}$ & $3.75^{*} 10^{-6}$ & $1.8 * 10^{-5}$ \\
\hline $\mathrm{ZnCl}_{2}$ & $6.25 * 10^{-6}$ & $3 * 10^{-5}$ \\
\hline $\mathrm{HBO}_{3}$ & $6.25 * 10^{-6}$ & $3 * 10^{-5}$ \\
\hline$\left(\mathrm{NH}_{4}\right)_{6} \mathrm{Mo}_{7} \mathrm{O}_{24} .4 \mathrm{H}_{2} \mathrm{O}$ & $1.12 * 10^{-5}$ & $5.4 * 10^{-5}$ \\
\hline $\mathrm{Na}_{2} \mathrm{SeO}_{3} .5 \mathrm{H}_{2} \mathrm{O}$ & $1.25 * 10^{-5}$ & $6 * 10^{-5}$ \\
\hline $\mathrm{NiCl}_{2} .6 \mathrm{H}_{2} \mathrm{O}$ & $6.25 * 10^{-6}$ & $3 * 10^{-5}$ \\
\hline EDTA (tripex 2) & $1.25 * 10^{-4}$ & $6 * 10^{-4}$ \\
\hline Na-Resazurin & $6.25 * 10^{-5}$ & $3 * 10^{-4}$ \\
\hline $\mathrm{HCl} 36 \%$ & $5.4 * 10^{-5}$ & $7.2 * 10^{-4}$ \\
\hline \multicolumn{3}{|l|}{ Vitamins } \\
\hline p-aminobenzoate (Na-salt) & $4 * 10^{-7}$ & - \\
\hline biotin (vitamin $\mathrm{H}$ ) & $8 * 10^{-8}$ & - \\
\hline cyanocobalamin (vitamin B12) & $8 * 10^{-7}$ & - \\
\hline nicotin acid & $8 * 10^{-7}$ & - \\
\hline panthothenate (Na-salt) & $2 * 10^{-7}$ & - \\
\hline pyridoxine (vitamin B6) & $2 * 10^{-6}$ & - \\
\hline thiamine $\mathrm{HCl}$ (vitamin B1) & $4 * 10^{-7}$ & - \\
\hline
\end{tabular}


Table S5.2: Input values in Homogeneous Surface Diffusion Model.

\begin{tabular}{lrr}
\hline Parameter & Value & Unit \\
\hline Bed length & 0.1 & $\mathrm{~m}$ \\
Bed density & 445 & $\mathrm{~g} / \mathrm{L}$ \\
Bed porosity & 0.65 & - \\
Particle radius & 0.000675 & $\mathrm{~m}$ \\
Surface diffusion coefficient & $6.5^{*} \times 10^{-15}$ & $\mathrm{~m}^{2} / \mathrm{s}$ \\
Film diffusion coefficient & $1.6 * 10^{-6}$ & $\mathrm{~m} / \mathrm{s}$ \\
Freundlich affinity coefficient & 26 & $(\mathrm{~L} / \mathrm{g})$ \\
Freundlich intensity parameter & 0.39 & - \\
\hline
\end{tabular}

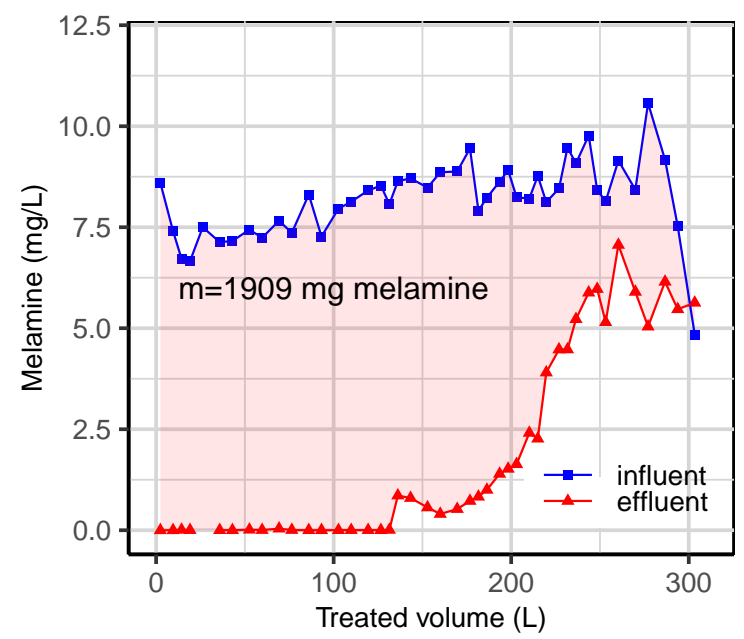

Figure S5.1: Amount of melamine removed in the inoculated filter BAC 1, based on influent and effluent concentration curves. 

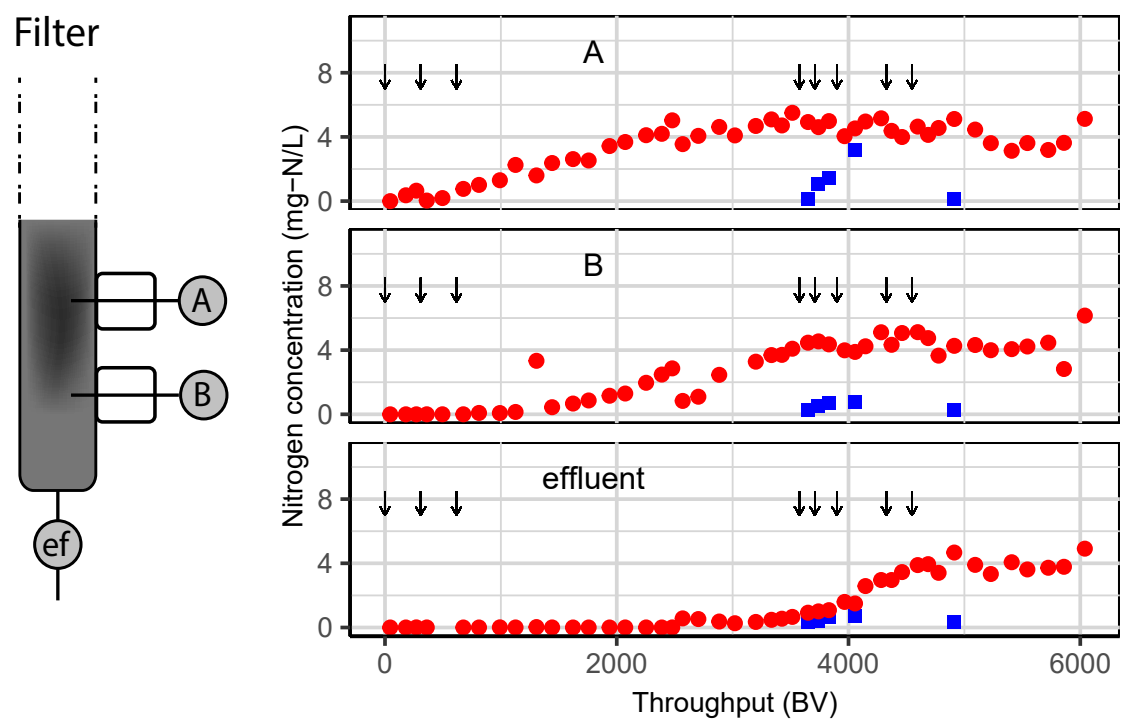

- melamine

ammonium

Figure S5.2: Nitrogen concentration in melamine and ammonium in intermediate sampling points (A and B) and effluent of inoculated filter without methanol. Arrows indicate inoculation of the filter. 


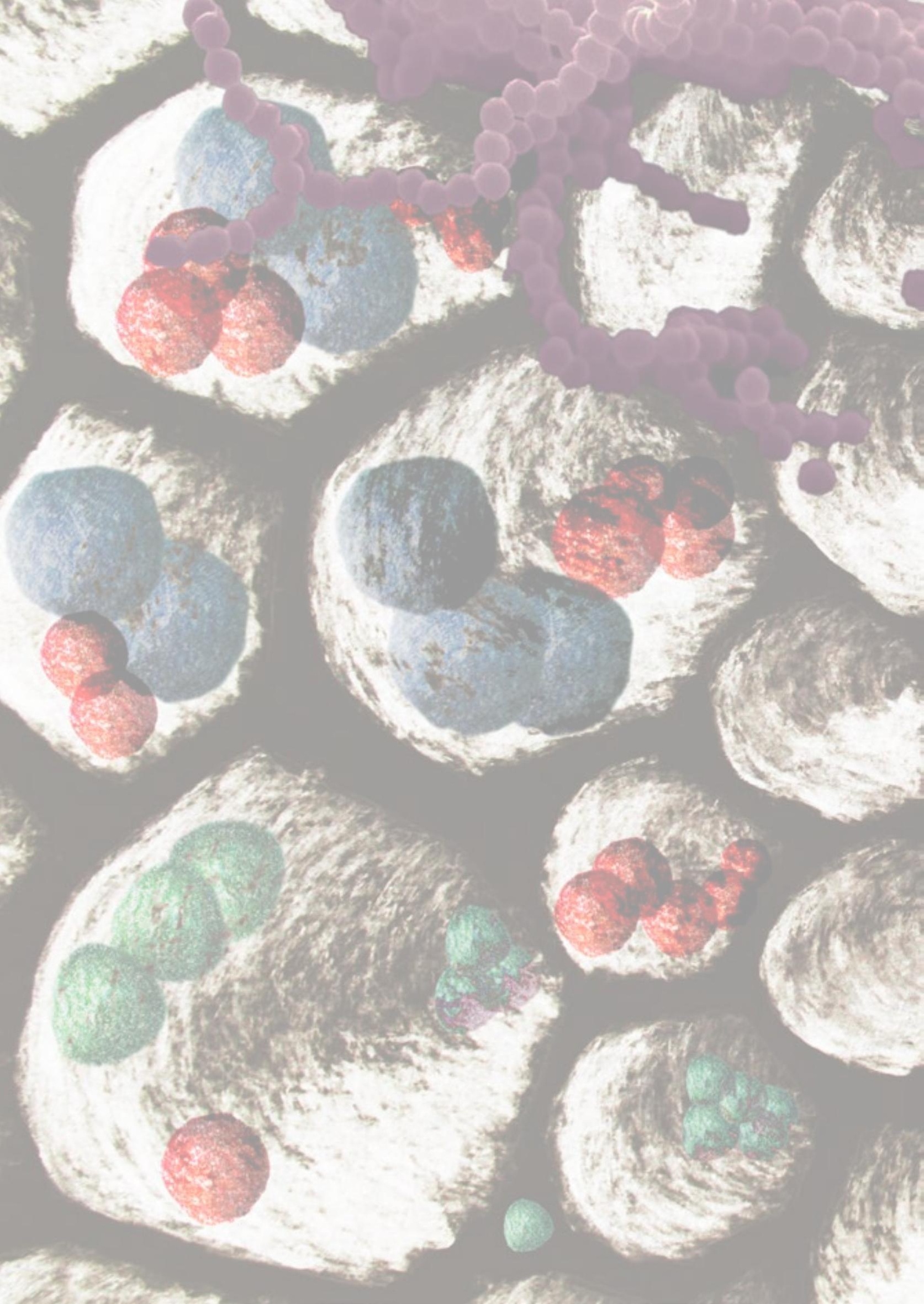




\subsection{Introduction}

This thesis describes the use of microbial activity to regenerate the adsorption capacity of granular activated carbon (GAC) used to remove micropollutants from water. Adsorption to GAC in filters is an effective technology to remove a number of micropollutants from water. However, maintaining high removal efficiencies requires frequent thermal reactivation in an energy intensive process, resulting in large carbon footprints of this technology (Mo et al., 2018; Mousel et al., 2017). Bioregeneration can reduce our dependency on unsustainable thermal reactivation methods.

In this thesis, the adsorption of small hydrophilic micropollutants by fresh and used GAC was assessed (Chapters 2 and 3). Additionally, the capacity of indigenous biomass grown in GAC filters to biodegrade micropollutants was investigated (hapter 3 ). Furthermore, the potential of applying bioregeneration to recover the adsorption capacity of GAC, loaded with one model micropollutant (melamine), was studied (Chapters 4 and 5 ). The present chapter presents considerations regarding the outcome of these experimental chapters, recommendations for future research and opportunities for using bioregeneration to improve water treatment.

\subsection{Bioregeneration of granular activated carbon}

\subsubsection{Assessing bioregeneration}

Bioregeneration of GAC consists of recovering GAC adsorption capacity by biodegrading previously adsorbed compounds (Aktaş and Çeçen, 2007). The concept of bioregeneration applies to GAC loaded with any class of adsorbent. In this thesis, the bioregeneration of GAC loaded with micropollutants was studied. This process is achieved through the following steps: when micropollutants in the liquid phase are biodegraded, previously adsorbed micropollutants desorb due to a concentration gradient from the GAC to the liquid phase. This process continues as long as biomass is active and micropollutants desorption can take place. Consequently, GAC adsorption sites previously occupied by micropollutants become available again and GAC adsorption capacity is regenerated.

Bioregeneration of GAC can be assessed both in batch and column studies. In general, batch studies require less materials, which often allows testing a larger number of parameters or conditions influencing the process. However, batch studies are less representative of filters where GAC is used. Therefore, also column studies are needed to assess bioregeneration in hydrodynamic conditions more similar to those of application. 
In this thesis, both approaches were used and different bioregeneration efficiencies were achieved in each one (Chapters 4 and 5). For example, a much higher bioregeneration efficiency for GAC loaded with melamine was obtained in columns (98\%) than in batches (28\%). This discrepancy is related to several factors. First, given the different experimental setups, a different strategy was used to quantify bioregeneration in each study (Figure 6.1). In the batches, bioregeneration was quantified based on the remaining GAC adsorption capacity after GAC treatment with active biomass, compared to a control which was exposed to inactive biomass. The experimental design caused regeneration also in the abiotic control due to desorption when transferring the loaded GAC to melamine-free media. The regeneration of GAC in the control resulted in an underestimation of the bioregeneration efficiency in the test condition. In the columns, bioregeneration was calculated solely based on the desorption of previously adsorbed melamine since GAC remaining adsorption capacity was not tested. With this approach, the resulting bioregeneration was $98 \%$. However, even though $98 \%$ of the previously adsorbed melamine desorbed, not necessarily $98 \%$ of the adsorption capacity was recovered, since fouling might have decreased the GAC adsorption capacity. Differently from the batches, this strategy might have resulted in an overestimation of bioregeneration.

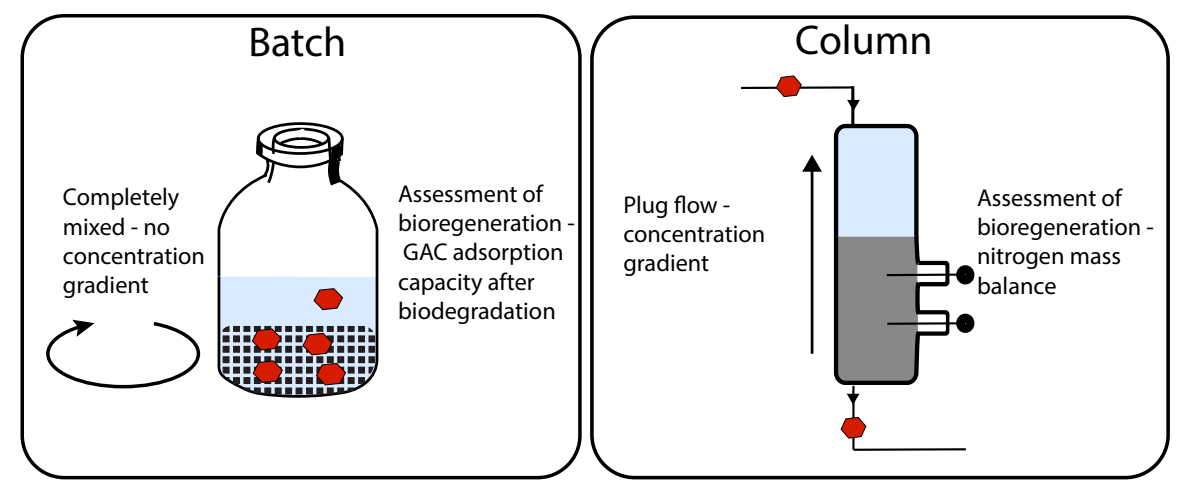

Figure 6.1: Different setups (batch, left, and column, right) used to assess GAC bioregeneration.

Second, the different hydraulic regimes in both studies likely influenced the extent of bioregeneration (Figure 6.1). In the batches, bioregeneration was assessed in a closed system. This means that the non-adsorbed fraction of degradation products remains in the liquid phase and can potentially decrease biodegradation rates. Additionally, the system is completely mixed so all GAC mass is in contact with the same melamine concentration. In the columns, the non-adsorbed fraction of degradation products washes out from the system, hence do not limit biodegradation rates. Moreover, the plug-flow leads to 
stratification of the filter bed, forming a concentration gradient in the liquid phase along the column length. This results in layers with a gradient in concentration, differently from a mixed system. The lower the adsorbate concentration in the liquid phase is, the more intense the driving force for desorption becomes (Speitel et al., 1987). This effect potentially resulted in higher bioregeneration efficiencies in the columns, as seen in this thesis.

The most appropriate means to assess the potential for bioregeneration in practice is using a setup with hydrodynamic conditions comparable to those of GAC applications, which are usually fixed-bed filters. Nevertheless, batch studies are useful to assess conditions influencing bioregeneration, e.g., GAC properties, biomass inoculation, amongst others. Batch studies are important as preliminary steps to minimize the number of variables to be tested in column studies.

\subsubsection{Micropollutants biodegradation}

Bioregeneration of GAC requires significant micropollutants biodegradation in the filters.

Micropollutants biodegradation is influenced by several factors, including the presence of organic matter and electron donors and acceptors in the influent, which determine which microbial processes can take place. Three of these processes, which are contemplated in this thesis, are discussed below.

\subsubsection{Cometabolic biodegradation by heterotrophic microorganisms}

Cometabolic biodegradation of micropollutants by heterotrophic microorganisms occurs when micropollutants are biodegraded exclusively in the presence of an additional carbon source. This mechanism might have taken place in the study presented in this thesis (Chapter 3), as the organic matter adsorbed to used GAC can serve as additional carbon source to microorganisms. Cometabolic biodegradation of micropollutants by heterotrophic microorganisms is likely to occur in GAC filters due to the presence of organic matter in the filter influent at much higher concentrations than the micropollutants (see section 6.3.2).

This thesis shows that an additional carbon source, such as methanol, increases melamine biodegradation (Chapters 4 and 5 ), as a consequence of microorganisms using melamine as a nitrogen source. In addition, melamine is also degraded in the absence of an additional carbon source, although to a lesser extent. Therefore, stimulated melamine biodegradation in the presence of methanol is not, by definition, a case of cometabolic biodegradation. 


\subsubsection{Cometabolic biodegradation by nitrifying microorganisms}

It is known that cometabolic biodegradation by nitrifying microorganisms plays a role in the removal of a variety of micropollutants, including diclofenac and iopromide (Batt et al., 2006; Dawas-Massalha et al., 2014; Suarez et al., 2010). In the study presented in this thesis (Chapter 3), ammonium was present in the media and the experiment was conducted in oxic conditions, hence nitrification might have played a role in iopromide biodegradation in this study. However, nitrification is not expected to happen in GAC filters in drinking water treatment since ammonium is only present in this water stream at trace concentrations $(<3$ $\mu \mathrm{g} / \mathrm{L}$, Lautenschlager et al., 2014). When GAC filters are used in wastewater treatment, they are usually applied after secondary or tertiary treatment and typical ammonium concentrations in this stream are also relatively low $(<0.2 \mathrm{mg}-\mathrm{N} / \mathrm{L}$, Altmann et al., 2016). Therefore, nitrification will not play a role in micropollutants removal in GAC filters used in wastewater treatment either.

\subsubsection{Redox conditions}

Redox conditions play a crucial part in microbial metabolism and consequently on micropollutants biodegradation. In this thesis, micropollutants biodegradation in both oxic (for iopamidol, iopromide, metformin and melamine) and anoxic (for melamine) conditions is reported. It is expected that most micropollutants are biodegraded exclusively or at least at higher rates in oxic conditions (Falås et al., 2013; Suarez et al., 2010), hence oxygen can be dosed to stimulate micropollutants biodegradation (Sperlich et al., 2017). Nevertheless, studies show the iodinated contrast agent iopromide is also biodegraded in anoxic conditions (Redeker et al., 2018). Additionally, faster biodegradation of guanylurea and melamine in anoxic than in oxic conditions has been observed (Chapter 4; Tisler and Zwiener, 2019). Micropollutants biodegradation in anoxic conditions broadens opportunities for combining both micropollutants and nutrients removal from water, as anoxic steps are commonly applied to remove nitrogen and phosphorous in wastewater treatment (Winkler and Straka, 2019). Filters combining micropollutants removal and denitrification for treating secondary wastewater effluent are studied already in pilot-scale, where methanol is dosed to stimulate both denitrification and micropollutants biodegradation (de Jong et al., 2018). Furthermore, in Chapter 4 , simultaneous melamine and nitrogen removal were combined in alternating nitrification and denitrification steps.

Oxic and anoxic conditions can be present at the same time in a GAC filter at different layers due to a vertical stratification of the filter bed resulting from the plug flow. The presence of 
oxic and anoxic conditions in one system increases microbial diversity, potentially increasing the number of biodegraded micropollutants (Grandclément et al., 2017).

\subsubsection{Micropollutants biodegradation in activated carbon filters}

Bioregeneration of GAC loaded with micropollutants can only be achieved when biomass capable of degrading these contaminants is present. The potential of indigenous biomass from GAC filters to degrade micropollutants is described in Chapter 3. Metformin, iopromide and iopamidol were biodegraded in the presence of nutrients and at high micropollutants concentrations $(>25 \mu \mathrm{M})$. In full scale GAC filters, the conditions for micropollutants biodegradation are less favourable, since both nutrients and micropollutants are present at lower concentrations in the filters influent. Nevertheless, biodegradation of several micropollutants in bench- to pilot-scale GAC filters by indigenous biomass has been reported (Altmann et al., 2016; Greenstein et al., 2018; Zhang et al., 2017; Zhiteneva et al., 2020). These studies indicate that micropollutants are removed in full-scale GAC filters not only through adsorption but also through biodegradation, although the relative contribution of each process is unknown.

In general, it is assumed for biodegradable compounds that adsorption is more relevant in the beginning of the filter operation, or immediately after GAC reactivation. Biodegradation becomes more relevant as adsorption capacity reduces and the biofilm on the GAC develops (Wang et al., 2007). Therefore, analysing the micropollutants concentration trend in the filter effluent allows to infer the removal mechanisms. For instance, if the concentration of a micropollutant in the filter effluent reaches influent levels, this micropollutant is not biodegraded (Figure 6.2a). If the micropollutant removal reaches a steady-state concentration below influent levels, this is a strong indication that biodegradation is the main removal mechanism in the steady-state phase (Figure 6.2b) (Gibert et al., 2013). Finally, a situation can occur in which biomass adaptation or changes in filter conditions leads to increased biodegradation rates and a decrease in effluent concentrations (Figure 6.2c). This trend can be observed in the study presented in $\underline{\text { Chapter } 5}$ once methanol was supplied to a filter after breakthrough. 

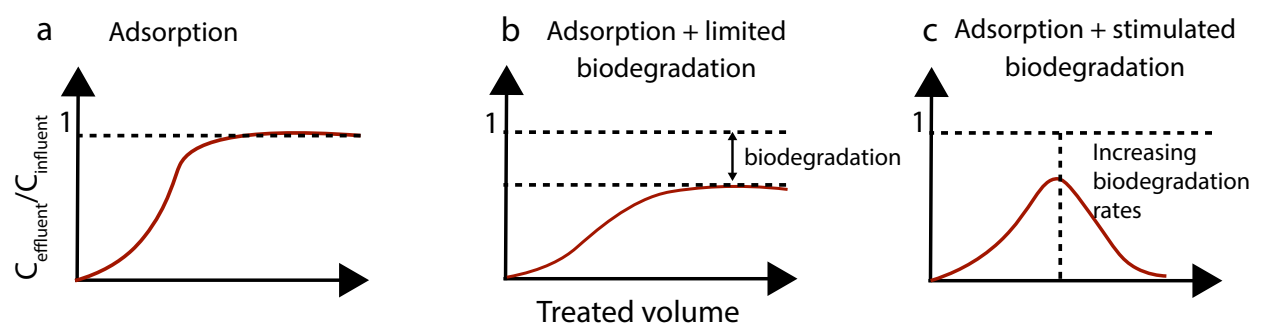

Figure 6.2: Removal trends in GAC filters.

In order to assess the extent of micropollutants biodegradation in GAC filters or other system with activated carbon (AC), it is necessary to quantify removal due to biodegradation or adsorption separately. Possible means of achieving this are through mass balances with inorganic non-adsorbable degradation products, e.g., ammonium and nitrate (Chapter 5; Smolin et al. 2020). Alternatively, mass balances can be calculated based on carbon, using isotope labelled substrates (Putz et al., 2005; Speitel et al., 1987). Moreover, adsorption can be assessed by quantifying the micropollutants on the GAC phase (Herzberg et al., 2003; Nguyen et al., 2016), although uncertainties regarding the recovery efficiency when extracting adsorbates from GAC limit the applicability of this strategy. Quantifying micropollutants biodegradation in the presence of GAC is important to determine the relevance of this removal mechanism and assess the extent of bioregeneration.

\subsubsection{Enhancing micropollutants biodegradation}

Bioregeneration of GAC requires significant micropollutants biodegradation. This can be achieved by stimulating activity of the indigenous microbial community (Chapter 3 ), or by adding exogenous microorganisms capable of degrading the targeted micropollutants (Chapters 4 and 5), a method known as bioaugmentation. In environmental microbiology, it is generally assumed that "everything is everywhere, but the environment selects", as proposed by the Dutch microbiologist Baas Becking in 1934 (translated in de Wit and Bouvier, 2006). In this perspective, providing suitable conditions for microbial activity should suffice to obtain micropollutants biodegradation.

Micropollutants are present in GAC filters at trace concentrations, hence their degradation provides little to no energy for microorganisms. Therefore, there is limited selective pressure for metabolic biodegradation of micropollutants by the indigenous microbial community in GAC filters, especially if the micropollutants are only intermittently present in the influent. Bioaugmentation is a valid alternative when the ability of degrading a target micropollutant 
is not expressed by the indigenous biomass (Benner et al., 2013). Increased micropollutants removal in biological treatment after bioaugmentation with pure and mixed cultures or with acclimatized activated sludge has been demonstrated in previous research (Albers et al., 2015; Boonnorat et al., 2018; Herrero and Stuckey, 2015; Iasur-Kruh et al., 2011).

In this thesis, bioaugmentation was successfully applied to regenerate lab-scale GAC filters treating influent containing the micropollutant melamine (Chapter 5). First, the ability of the indigenous biomass grown in GAC filters to degrade melamine was assessed (hapter 3 ). Melamine was not biodegraded by this inoculum using normal media (Chapter 3) or nitrogen free media (data not shown). Melamine biodegradation was only observed when inoculating the experiments with biomass from an industrial wastewater treatment plant treating influent containing melamine (Chapters 4 and 5). These results suggest that the use of specialized biomass has a large potential to increase micropollutants degradation when the indigenous biomass does not possess this ability. However, even though inoculation with adapted biomass introduces micropollutants degraders to a filter community, biodegradation capacity will only be expressed if suitable conditions are applied.

Even though bioaugmentation has the potential to increase micropollutants removal in water treatment, the variety of compounds present in the filter influent and the fluctuation in their concentrations makes it challenging to determine or identify a suitable inoculum. Additionally, safety issues might arise when considering introducing exogenous biomass in final steps of drinking water treatment. Given the challenges involving bioaugmentation, stimulating activity of the indigenous microbial community is the preferable approach to achieve bioregeneration in GAC filters in drinking water treatment plants. This strategy would take advantage of the high metabolic diversity of microbial communities in GAC filters (Oh et al., 2018).

\subsection{Natural organic matter and micropollutants removal with activated carbon}

Natural organic matter (NOM) present in the filter influent impacts micropollutants adsorption to and desorption from GAC, as well as micropollutants availability and degradability in GAC filters. In this thesis, the effect of NOM on micropollutants removal is contemplated in Chapter 3, where adsorption capacity of used and fresh GAC was compared. In the bioregeneration studies (Chapters 4 and 5), preference was given to a simplified system, where only the interactions between micropollutants, biomass and GAC 
took place. Therefore, the effect of NOM on GAC bioregeneration was not included in these studies. In practice, NOM will always be present in the filter influent at various concentrations and compositions, resulting in different effects on micropollutants removal and GAC bioregeneration.

\subsubsection{Natural organic matter and micropollutants adsorption}

NOM is listed as one of the most relevant group of compounds in the water matrix determining efficiency of GAC filtration for micropollutants removal (Fischer et al., 2019). The relevance of NOM in determining GAC adsorption capacity for micropollutants is a consequence of NOM presence in water at much higher concentrations (mg/L range) than the micropollutants (ng/L - $\mu \mathrm{g} / \mathrm{L}$ range) (Zietzschmann et al., 2016b). NOM can reduce micropollutants adsorption to GAC by direct competition for adsorption sites or pore blockage (Li et al., 2003a), which prevents micropollutants from accessing the adsorption sites. With the organic matter present in wastewater effluent, competition for adsorption sites is considered more relevant than pore blockage for reducing micropollutants adsorption ( $\mathrm{Hu}$ et al., 2016; Zietzschmann et al., 2014). Pore blockage can also prevent micropollutants desorption (Pelekani and Snoeyink, 1999), limiting GAC bioregeneration. However, the effect of NOM on micropollutants adsorption rate is debatable (To et al., 2008a). Some authors demonstrate that pore blockage or pore constriction can reduce adsorption kinetics due to a lower intra-particle diffusion (Li et al., 2003a) whereas other studies observe an increase in adsorption and desorption kinetics in the presence of NOM (To et al., 2008b). The apparent contradicting effects are related to the two mechanisms of intra-particle diffusion. Adsorbates can diffuse in the GAC pores either in the adsorbed state, through the GAC surface (surface diffusion) or in the dissolved state, through the pore liquid (pore diffusion). Adsorption of NOM increases surface diffusion as a consequence of the increased GAC surface coverage (To et al., 2008b), while decreasing pore diffusion, as a consequence of the increased pore tortuosity (Kennedy and Summers, 2015).

It is important to understand which properties of NOM are responsible for each of these mechanisms in order to minimize the negative effects on micropollutants adsorption and desorption. NOM consists of adsorbable and non-adsorbable fractions and only the first fraction influences micropollutants adsorption to AC (Shimabuku et al., 2017a; Wigton and Kilduff, 2004). This influence can be correlated to NOM molecular size rather than NOM chemical properties, such as aromaticity, polarity, specific UV absorbance and acidity (Shimabuku et al., 2017a; Velten et al., 2011b; Wigton and Kilduff, 2004). Previous research 
has shown that smaller molecules of NOM can access AC pores in the same size range as micropollutants, thus reducing adsorption due to competition for adsorption sites. Larger molecules of NOM, which adsorb mainly in larger pores than micropollutants, reduce adsorption capacity for the latter by blocking AC pores (Aschermann et al., 2019a; Li et al., 2003a; Pelekani and Snoeyink, 1999).

AC properties also influence the extent of these interactions, being pore size distribution the most important predictor (Pelekani and Snoeyink, 1999). However, different conclusions have been reached by studies with NOM present in surface and groundwater or with organic matter present in wastewater effluent (Hu et al., 2016; Li et al., 2003a, 2003b; Zietzschmann et al., 2014). Previous research with surface and groundwater has shown that the effect of NOM on micropollutants adsorption is stronger when using microporous activated carbon. When using AC with larger micropores and mesopores, micropollutants adsorb to the same extent both in the presence or absence of NOM ( $\mathrm{Li}$ et al., 2003a). On the contrary, with organic matter from wastewater effluent, the detrimental effects of GAC preloading on micropollutants adsorption were stronger in GAC with larger micropores and mesopores (Hu et al., 2016; Zietzschmann et al., 2014). Since the NOM present in the influent of GAC filters in drinking water treatment originates from surface water, a more open pore structure is expected to reduce the impact of NOM on micropollutants adsorption.

Reduced micropollutants adsorption in the presence of NOM does not necessarily reduce the potential for GAC bioregeneration. GAC bioregeneration can be negatively impacted by NOM only if pore blockage prevents micropollutants desorption. This effect is more influenced by GAC pore size distribution than by NOM composition (Aschermann et al., 2019b, 2019a). Aschermann et al. (2019b) compared micropollutants adsorption using ACs with different pore size distributions and concluded that pore blockage limited micropollutants desorption from microporous AC, whereas adsorption was still fully reversible in mesoporous AC. Interestingly, an increased desorption was observed with macroporous AC, which was attributed to micropollutants displacement by the organic matter. However, AC pore size distribution could not explain results obtained in a later study and the authors suggest that NOM can lead to irreversible micropollutants adsorption through unknown mechanisms other than pore blockage (Aschermann et al., 2019a).

This thesis and previous research (hapter 3; Aschermann et al., 2018; de Ridder et al., 2011) show that fresh GAC can adsorb hydrophilic micropollutants and the decrease in GAC adsorption capacity is mainly due to adsorption of NOM. This reduced adsorption capacity 
due to NOM adsorption explains why compounds like melamine and iopromide adsorb relatively well to fresh GAC ( $\underline{\text { Chapter } 2)}$ whereas they are reported as poorly removed in pilot scale GAC filters (Brunner et al., 2020; Kennedy et al., 2015). To illustrate this effect, the time required to reach breakthrough in full-scale GAC filters was calculated, considering only the presence of these two micropollutants (i.e., without competition with NOM) at 1 $\mu \mathrm{g} / \mathrm{L}$ (melamine) and $10 \mu \mathrm{g} / \mathrm{L}$ (iopromide and melamine). The breakthrough curves were calculated using the adsorption model applied in Chapter 5, combined with the isotherm coefficients presented in Chapter 2. The filter dimension and flow used for the calculations were those of the drinking water treatment plant located in Kralingen, the Netherlands, operated by the water company Evides. The breakthrough curves are depicted in Figure 6.3.

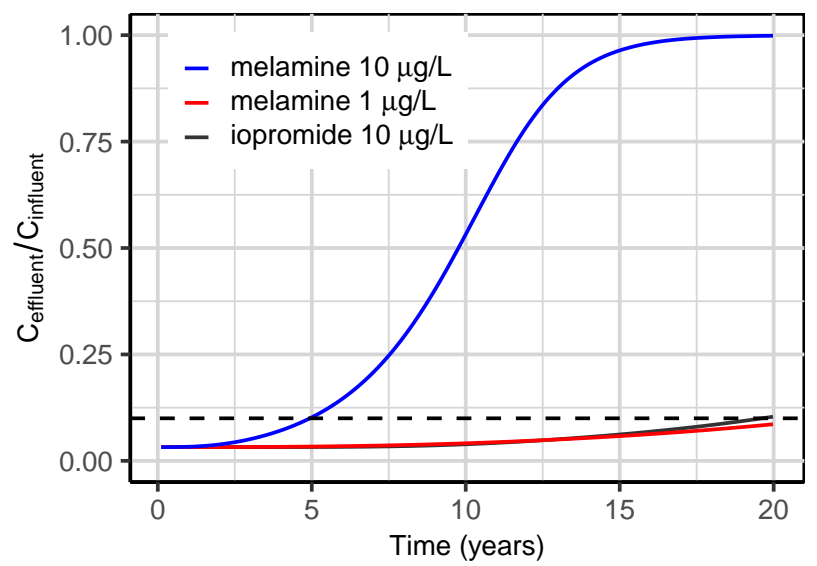

Figure 6.3: Calculated micropollutants breakthrough curves in a full-scale GAC filter for an influent concentration of $1 \mu \mathrm{g} / \mathrm{L}$ (melamine) and $10 \mu \mathrm{g} / \mathrm{L}$ (iopromide and melamine), in the absence of competition with natural organic matter or other organic contaminants. Dashed line indicates $10 \%$ breakthrough.

The model simulation shows that when melamine and iopromide are present in the filter influent at concentrations of $1 \mu \mathrm{g} / \mathrm{L}$ and $10 \mu \mathrm{g} / \mathrm{L}$ respectively, only $10 \%$ breakthrough occurs within 20 years. Even when melamine is present at $10 \mu \mathrm{g} / \mathrm{L}$, removal remains above $90 \%$ for 5 years. Interestingly, negligible melamine removal in pilot-scale GAC filters has been reported (Brunner et al., 2020). These results show that exhaustion of GAC in filters occurs mainly due to adsorption of NOM and other organic contaminants present at higher concentrations, and not to adsorption of micropollutants. Previous research has shown that in the concentration range in which micropollutants occur, competition between the micropollutants does not seem to affect the adsorption capacity for an individual compound (Kennedy and Summers, 2015). Therefore, even though the filters influent contains a 
mixture of several micropollutants, it is reasonable to calculate the breakthrough for an individual compound disregarding the presence of other micropollutants.

To conclude, increasing micropollutants adsorption in GAC filters can be achieved by minimizing detrimental effects from NOM adsorption. This can be achieved by removing in upstream processes the fraction of NOM that interferes the most with micropollutants adsorption, i.e., the low molecular weight adsorbable fraction. Alternatively, the impact of NOM can be reduced by carefully selecting the GAC based on its pore size distribution. It is known that microporous GACs have higher affinity for micropollutants (Chapter 2; Lu and Sorial, 2004). However, GAC microporosity increases the negative effects of NOM adsorption on micropollutants removal, whereas GACs with a wider range of pore sizes minimize these effects ( $\mathrm{Li}$ et al., 2003b). If adsorption of micropollutants is to be optimized in GAC filters, subsequent filters with GACs with different pore size distribution can be applied in sequential steps. A first filtration step using GAC with a more open pore structure will remove a large fraction of the background NOM (Aschermann et al., 2019a; Velten et al., 2011b). A second filtration step would then target the adsorption of micropollutants.

\subsubsection{Natural organic matter and micropollutants biodegradation}

NOM is a complex mixture of organic compounds and its different fractions have a different biodegradability (Chen et al., 2016). Approximately $22 \%$ to $32 \%$ of the NOM is removed through biodegradation in GAC filters (Han et al., 2013; Velten et al., 2011a). Additionally, biodegradability of NOM present in GAC filters varies according to the NOM source and water treatment steps applied before GAC filtration (Chen et al., 2016). For instance, NOM biodegradability increases after oxidation steps commonly applied in water treatment trains before GAC filtration, such as ozonation (Matilainen et al., 2006) and UV irradiation (Buchanan et al., 2004; Thomson et al., 2002) with potential beneficial effects to GAC bioregeneration.

It can be expected that the biodegradable fraction of NOM stimulates micropollutants biodegradation through cometabolism (see 6.2.2.1). Cometabolic biodegradation, instead of metabolic biodegradation, is considered as the prevailing mechanism for micropollutants biodegradation at environmentally relevant concentrations (Fischer and Majewsky, 2014). However, research shows that this is not always the case and the role of NOM on micropollutants biodegradation depends on NOM composition and on the micropollutants and systems studied. 
In the context of wastewater treatment, studies with easily degradable carbon sources (acetate), raw wastewater and humic acids show that the effect of additional organic carbon on micropollutants biodegradation is compound-dependent (Nord and Bester, 2020; Tang et al., 2017; Zhang et al., 2019). While biodegradation of certain micropollutants, including diclofenac and iopromide, increases with supply of organic carbon, biodegradation rates of other micropollutants remain unaffected. Furthermore, studies of micropollutants biodegradation in wastewater treatment also suggest that limitation of the growth substrate positively correlates to higher micropollutants removal (Grandclément et al., 2017).

Previous research assessed whether additional carbon sources can increase micropollutants biodegradation rates in biofilters and simulated aquifer recharge systems (Alidina et al., 2014; Hellauer et al., 2019; Ma et al., 2018). A positive correlation between influent dissolved organic carbon and micropollutants removal in pilot-scale GAC-sand filters has been reported (Ma et al., 2018). Furthermore, these studies show that composition of NOM, besides its concentration, is also important to determine its role on micropollutants biodegradation. Alidina et al. (2014) observed that a refractory carbon substrate (humic acids) increases microbial diversity, which can increase the potential for micropollutants biodegradation in simulated managed aquifer recharge systems. However, conflicting results were observed by Hellauer et al. (2019), who observed no effect of increased humic acids concentration on removal of biodegradable micropollutants in managed aquifer recharge systems. Based on their findings and literature, they concluded that the microbial community can adapt to low organic carbon concentrations $(<1 \mathrm{mg} / \mathrm{L}$ dissolved organic carbon) and biodegrade micropollutants metabolically, although these effects are also micropollutant-dependent.

Adaptation of a microbial community to oligotrophic conditions is a key factor determining the effect of additional carbon sources on micropollutants biodegradation. This reinforces the importance of investigating the effects of NOM on micropollutants biodegradation in GAC filters. GAC filters are different from other biofilters because of the GAC high adsorption capacity, which results in substrate adsorption to the filter media, separating hydraulic retention time from substrate retention time. The integration of adsorption and therefore substrate accumulation on the filter media can impact the influence of NOM on micropollutants biodegradability. Future research should elucidate the effect of NOM concentration and composition on cometabolic biodegradation micropollutants in GAC filters. 


\subsubsection{Influence of natural organic matter on combined adsorption and biodegradation}

On the one hand, NOM can potentially increase micropollutants biodegradability by promoting cometabolic biodegradation (6.3.2). On the other hand, NOM can limit GAC bioregeneration by hindering micropollutants desorption (6.3.1). The success of bioregeneration will depend on maximizing the positive influence of NOM on micropollutants biodegradation whilst minimizing its impact in micropollutants desorption.

Selecting GAC with an open pore structure will help to minimize desorption hysteresis. Furthermore, the intensity of desorption hysteresis correlates to the micropollutants affinity for GAC (Aschermann et al., 2019b). Therefore, this effect is expected to be limited for a large number of micropollutants relevant for drinking water production, which are known to weakly adsorb to GAC. Ideally, GAC bioregeneration can be optimized by maintaining in the filter influent NOM that promotes micropollutants biodegradation and selecting GAC with a pore size distribution that allows for full micropollutants desorption.

\subsection{Micropollutants concentration}

Micropollutants concentrations used in this thesis were higher than those found in domestic wastewater and surface water used for drinking water production. The reasons for that choice were several, including the detection limits of analytical methods and the design of most experiments. Some experiments required micropollutants concentrations high enough to reach GAC saturation (Chapter 4). Other experiments would be unfeasible within the 4 years' time-frame of this study if environmentally relevant concentrations were used (Chapter 5). To illustrate this, the Homogeneous Surface Diffusion Model presented in Chapter 5 was used to estimate the time required to reach breakthrough in the lab-scale GAC filters. The effluent breakthrough curves were calculated for influent containing melamine concentrations ranging from $10 \mu \mathrm{g} / \mathrm{L}$ to $10 \mathrm{mg} / \mathrm{L}$, for a contact time of 30 minutes. The results show that whereas $100 \%$ breakthrough is obtained within 3 months when melamine influent concentrations are $10 \mathrm{mg} / \mathrm{L}$, no breakthrough is observed within one year if influent concentrations were reduced to $100 \mu \mathrm{g} / \mathrm{L}$ or lower (Figure 6.4). As a result of time constraints, higher melamine concentrations were used in this thesis. 


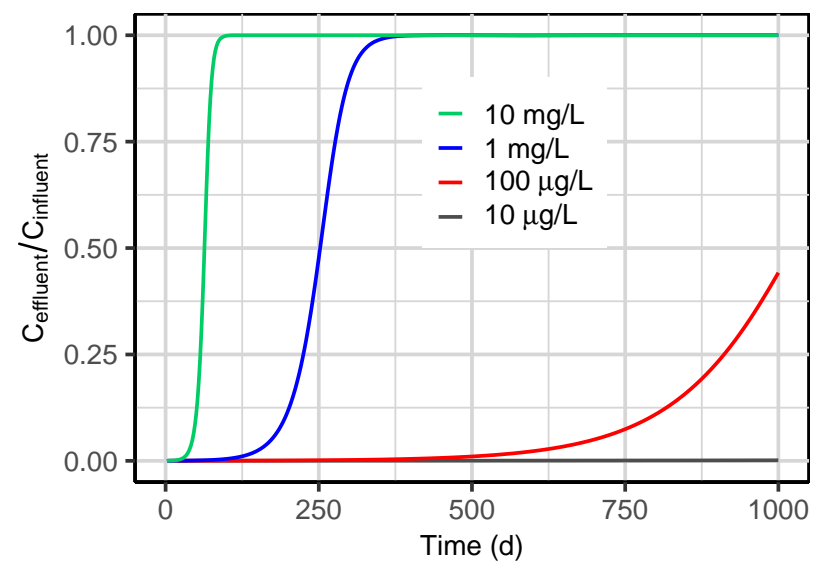

Figure 6.4: Calculated breakthrough curves of melamine in lab-scale GAC filters at different influent concentrations.

The reason for the late breakthrough in the modelled results is related to the absence of the effects of organic matter on melamine adsorption. In the presence of organic matter, GAC adsorption capacity for micropollutants is reduced (see 6.3.1). Therefore, the option of not including organic matter in a study also impacts the range of micropollutants concentration that are feasible, leading to the use of higher concentrations.

The choice of micropollutants concentrations in a study can influence the conclusions regarding their biodegradability, as at higher concentrations, metabolic biodegradation can also occur. However, micropollutants biodegradation at trace concentrations can benefit from the presence of GAC due to the accumulation of micropollutants on the GAC surface. This accumulation potentially compensates for or reduces impacts of mass transfer limitation and insufficient enzymatic affinities observed at low substrate concentrations (Blair et al., 2015; Joss et al., 2006). Modelling studies conclude that biofilm attached to GAC experiences a dual flux of substrate, both from the liquid and from the solid (GAC) phase when adsorbates desorb (Herzberg et al., 2003; Speitel et al., 1987). This dual flux allows maintaining active biomass at deeper biofilm depths, which would not be possible with inert carrier materials (Herzberg et al., 2003). These studies indicate that the flux of substrate from the GAC phase to the biofilm is more important than the flux from the liquid phase, hence, most of the degraded substrate originates from desorption from the GAC.

Micropollutants adsorption and biodegradation in GAC filters and GAC bioregeneration can be studied using lower concentrations of micropollutants when organic matter is 
included in the scope of the study. In this thesis, the feasibility of bioregeneration in the absence of NOM was demonstrated. In order to apply bioregeneration in full scale, future research should focus on assessing bioregeneration at lower micropollutants concentration in the presence of NOM.

\subsection{Modelling micropollutants removal in granular activated carbon filters}

\subsubsection{Modelling micropollutants adsorption with granular activated carbon}

Mathematical models describing the adsorption of contaminants are well established and useful for predicting breakthrough in fixed-bed filters (Chapter 5; Zhang et al., 2009). Micropollutants adsorption can be described using models based on diffusion (Chapters 2 and 5). In these models, the rate limiting steps for micropollutants adsorption are the diffusion in the stagnant film layer around the granule (film diffusion) and inside the granule (intraparticle diffusion). Micropollutants can diffuse in the granules either in the adsorbed phase (surface diffusion) or in the dissolved phase in the pore liquid (pore diffusion) (Figure 6.5).

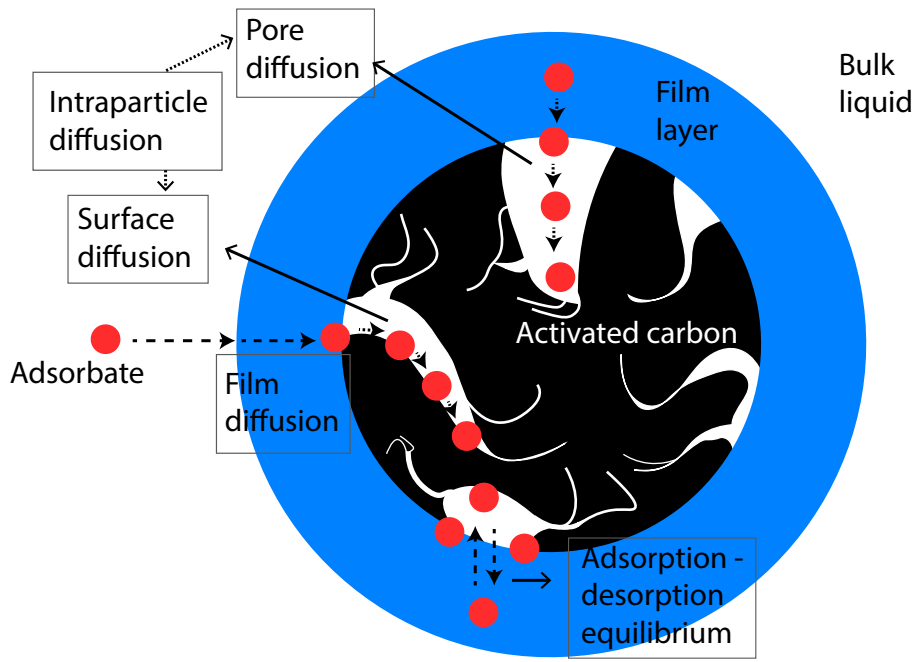

Figure 6.5: Adsorbate diffusion in the film layer and in the GAC particle. Adapted from Çeçen and Aktaş (2012).

Both pore diffusion and surface diffusion can be implemented in adsorption models to describe the adsorption kinetics (Chapters 2 and 5; Valderrama et al., 2008). In practice, surface and pore diffusion occur simultaneously and the intraparticle diffusion coefficient 
calculated with the models is the apparent intraparticle diffusion, resulting from the combination of both processes (Shen et al., 2012). Furthermore, the partition of the adsorbate between liquid phase and solid phase (GAC) in equilibrium is described in the models by an adsorption isotherm. In this thesis, the Langmuir isotherm was incorporated in the pore diffusion model, which gives a good fit with the adsorption kinetics obtained experimentally (Chapter 2). In addition, the Freundlich isotherm was also used in the pore diffusion model and the model output using both Langmuir and Freundlich equations was compared. For a number of micropollutants, both equations resulted in similar pore diffusion coefficients (data not shown). However, in Chapter 5 , the experimental results were represented better using the Freundlich isotherm in the Homogeneous Surface Diffusion Model, rather than the Langmuir isotherm. This is likely a consequence of an underestimation of the maximum adsorption capacity of melamine when using the Langmuir isotherm. Another possible reason is that the assumption of homogeneous adsorption sites by the Langmuir isotherm is not valid for melamine adsorption onto GAC. These results show that a good fitting of an isotherm equation to an experimental dataset does not always result in usefulness of this isotherm for modelling fixed-bed adsorbers.

Despite their predictive power, applicability of adsorption models in practice is limited given the influence of biomass on the processes occurring in GAC filters. This influence include not only contaminants removal through biodegradation, but also effects on adsorption kinetics due to mass transfer limitations. Therefore, in order to understand micropollutants removal in GAC filters and explore the potential of biodegradation to regenerate GAC, it is crucial to understand the interactions between all processes occurring in the filters.

\subsubsection{Modelling micropollutants removal with biologically active granular activated carbon}

Modelling adsorption in biologically active GAC (from now on referred to as bGAC) requires a thorough understanding of the processes occurring in the biofilm and a proper characterization of this compartment. Without the biofilm, adsorption models describe mass transfer in two compartments, the stagnant water layer around the GAC (film layer) and the GAC itself. Furthermore, these models assume that adsorbate removal occurs exclusively through adsorption to the GAC. When modelling bGAC, the third compartment (biofilm) adds a layer of complexity to the models, given that biofilms cause mass transfer resistance and can remove adsorbates through biodegradation and sorption (Figure 6.6) (Shen 2012). Sorption to the biofilm will not be taken into account in the next paragraphs, given that this 
process is not included in the bGAC models described in literature. Nevertheless, sorption to the biofilm will be addressed in the end of this section.

a
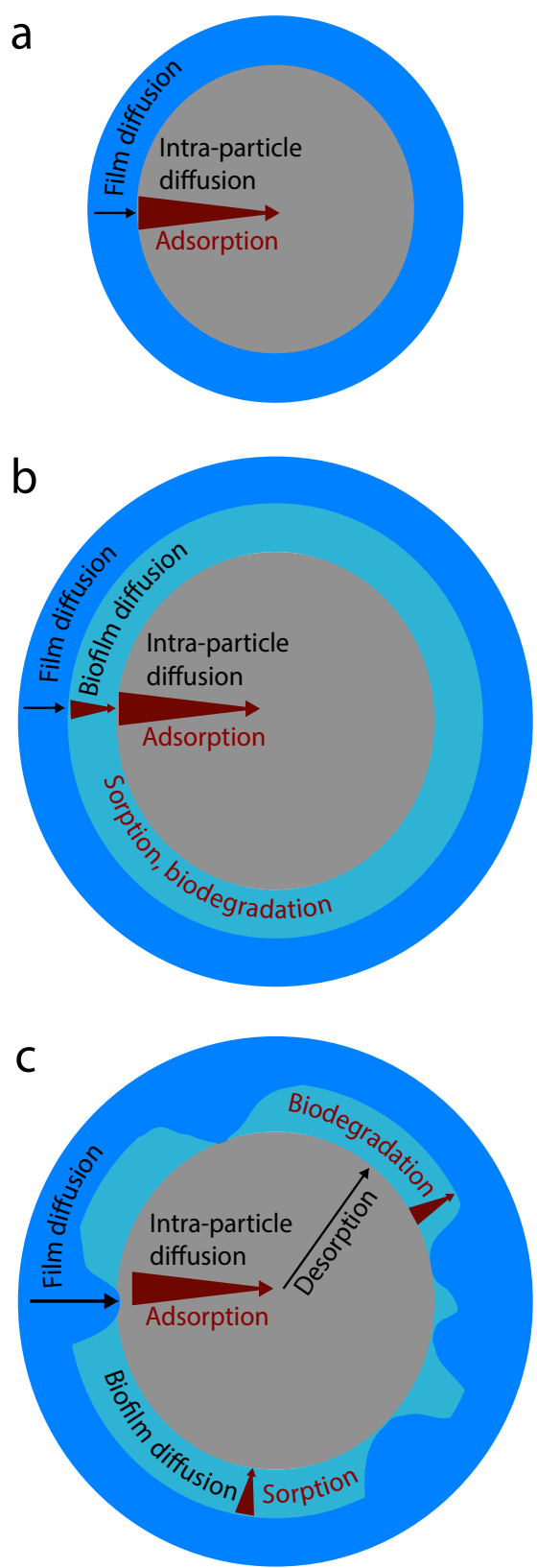

Figure 6.6: Mass transfer and removal processes occurring in GAC without biofilm (a) and with biofilm, considering full coverage (b) and partial coverage (c). 
To represent the processes occurring in the biofilm, a set of parameters and equations describing the biofilm itself, adsorbate transport and biodegradation in the biofilm is included in bGAC models. These parameters include biofilm thickness, biofilm growth and decay rates, adsorbate diffusivity and biodegradation in the biofilm. Speitel et al. (1987) were pioneers in presenting an adsorption and biodegradation model of a bGAC filter and assessing the model output against experimental data. The experimental results only partially matched the modelled results. The authors attributed the limited predictive power of the model to several factors, including the inaccurate calculation of a parameter representing biomass loss due to decay and shear. They concluded that more knowledge of the biofilm is needed to refine such models and increase their predictive power. Additionally, the authors concluded that the bGAC could be described more accurately by a scattered biofilm growth, rather than a biofilm fully covering the GAC particles, which is what most models assume (Shen et al., 2012).

The discontinuous biofilm coverage of bGAC was later discussed by Herzberg et al. (2003), who proposed a conceptual model with a patchy biofilm coverage. In this model, molecules reach the GAC and adsorb through the non-biofilm covered surface of the granules and diffuse back to the biofilm through the covered surface (Figure 6.6c). The authors conclude that if full biofilm coverage is assumed, adsorption cannot occur due to limited penetration of the adsorbate in the biofilm. Whilst the model with partial biofilm coverage is a more realistic representation of the bGAC and allows to describe mass transfer through different interfaces (GAClliquid and GAClbiofilm), it also introduces another set of parameters which cannot be determined experimentally nor calculated.

Obtaining reliable parameters for the processes occurring in the biofilm is one of the main challenges when building accurate models to predict micropollutants removal in a bGAC filter. Given that several biomass-related parameters cannot be experimentally determined nor calculated, they have to be determined by fitting experimental data to the model output. Consequently, these models describe the experimental data but have limited predictive power. For instance, in the study of Herzberg et al. (2003), biofilm diffusion coefficient, density and coverage fraction were determined by fitting experimental results. Despite their limitations, these models greatly contribute to understanding the interactions between biofilm and GAC, as well as quantifying micropollutants fluxes between these compartments and the liquid phase, and micropollutants removal in each compartment. 
To conclude, micropollutants adsorption in GAC filters can be predicted by fixed-bed adsorption models (Chapter 5). However, these models cannot describe systems where biofilms also determine micropollutants adsorption kinetics and removal, i.e., bGAC filters. In order to properly model bGAC and bioregeneration, more knowledge is needed regarding the biofilm formed around GAC. Currently, there is no single conceptual model for GAC bioregeneration, but various attempts with different assumptions. Additionally, no bGAC models published so far take into account micropollutants sorption to the biofilm, even though this might be a significant removal process (Chaumet et al., 2019; Ding et al., 2014; López-Ramón et al., 2019).

\section{Micropollutants sorption to biofilm}

In the context of biofilms, the term sorption is more commonly used, and more appropriate, than adsorption. Sorption comprises both adsorption and absorption. Adsorption refers to the accumulation of an adsorbate on the surface of an adsorbent. Absorption, on the other hand, is defined as the "transfer of a substance from one bulk phase to another bulk phase" (Worch, 2012), hence it is not limited to a surface process. Sorption sites in biofilms are not only present in the extracellular polymeric substances matrix, but also at the surface and inside microbial cells (Flemming, 1995). Therefore, the term sorption is a more accurate description for the accumulation of molecules in any of these compartments.

Sorption of several micropollutants to natural biofilms has been reported (Chaumet et al., 2019; Zhang et al., 2018), although the interactions governing this process and the main components of biofilms or microbial cells responsible for the sorption are poorly understood. Studies report no correlation between sorption to biofilms and micropollutants hydrophobicity (represented by $\log \mathrm{K}_{\text {ow/Dow }}$ ) (Torresi et al., 2017; Wunder et al., 2011). Micropollutants sorption to biofilms is mainly determined by electrostatic interactions. Positively charged micropollutants sorb to a larger extent than neutral or negatively charged compounds (Torresi et al., 2017; Wunder et al., 2011).

Even though micropollutants are expected to sorb to biofilms to a lesser extent than to AC, sorption to biofilms is a relevant process, especially for cationic micropollutants, and/or when biofilms are present at high densities. Furthermore, the relative importance of this process increases as the AC adsorption capacity reduces due to adsorption of other contaminants present at higher concentrations, e.g., NOM. More knowledge regarding micropollutants sorption to biofilms will allow assessing the relevance of this removal process and incorporate it in mathematical models of bGAC filters. 


\subsection{Recommendations for application}

\subsubsection{Mixed adsorbents to increase micropollutants removal}

This thesis and previous research (Chapters 2 and 3; de Ridder et al., 2010; Redding et al., 2009) show that the extent of micropollutants to AC depends on the micropollutants physico-chemical properties. Additionally, different adsorbent materials, including metal organic frameworks, chitosan, zeolites and $\beta$-cyclodextrin polymers (Bhadra and Jhung, 2017; Sompornpailin et al., 2020; Vakili et al., 2019) have been shown to have a comparable or even higher affinity for micropollutants than AC. Considering that surface water is contaminated with a large variety of micropollutants, with different physico-chemical properties, filtration with one single GAC type is most likely not suitable for all types of micropollutants. Hence, a combination of different types of GAC or even different adsorbent materials in the same or subsequent filters could contribute to increasing the number of micropollutants removed and their removal efficiency. In the case of mixing different types of adsorbents or types of GAC, it is crucial to consider not only the affinity of the adsorbent for different contaminants, but also its hydraulic properties, since combining different adsorbent materials can make filters operation more complex.

\subsubsection{Applicability of granular activated carbon bioregeneration to wastewater treatment}

This thesis assessed micropollutants biodegradation and GAC bioregeneration in the context of drinking water treatment. However, given the growing interest and great potential for applying GAC filters in wastewater treatment, the knowledge obtained in this thesis is also discussed in the context of this water stream. The removal of background organic matter from the filter influent, manipulation of filter conditions to promote microbial growth and biomass inoculation to the filters can be different for drinking water or wastewater applications.

Influent of GAC filters used in drinking water treatment contains a relatively lower concentration of organic matter with a more recalcitrant character (Zietzschmann et al., 2016a), as well as lower nutrients concentrations, in comparison to GAC filters in wastewater treatment plants (WWTP) (Table 6.1). Moreover, at similar dissolved organic carbon:micropollutants ratios, the organic matter in WWTP effluent seems to compete with adsorption of micropollutants more strongly than the NOM in drinking water (Zietzschmann et al., 2016a). As a consequence, micropollutants breakthrough occurs earlier in GAC filters used in WWTP (Zietzschmann et al., 2016b). This difference implies that the 
relative contributions of adsorption and biodegradation for micropollutants removal are different in GAC filters used in drinking water or wastewater treatment. It can be expected that adsorption will play a more important role in the first than in the later.

Table 6.1: Typical dissolved organic carbon (DOC), nitrogen and phosphorus concentrations in drinking water and treated domestic wastewater.

\begin{tabular}{lrr}
\hline Parameter & Drinking water* & Wastewater \\
\hline DOC $(\mathrm{mg} / \mathrm{L})$ & $0.96-2^{1,2}$ & $5-11.4^{3,4,5}$ \\
Nitrogen $(\mathrm{mg}-\mathrm{N} / \mathrm{L})$ & $0.9-2^{2,6}$ & $12-13.5^{3,7}$ \\
Phosphorus $(\mu \mathrm{g}-\mathrm{P} / \mathrm{L})$ & $<2-6^{6,8}$ & $500-800^{3,7}$ \\
\hline
\end{tabular}

*: values measured in GAC filters influent or earlier steps of drinking water treatment.

1: Velten et al. (2011b); 2: David de Ridder, personal communication, September 2020; 3: Altmann et al. (2016); 4: Bourgin et al. (2018); 5: Rattier et al. (2014); 6: Lautenschlager et al. (2014); 7: Scherrenberg et al. (2012); 8: Lehtola et al. (2001).

The relevance of biodegradation as a removal process in GAC filters treating domestic wastewater effluent has been demonstrated in previous research (Altmann et al., 2016; Bourgin et al., 2018; Reungoat et al., 2011). Reungoat et al. (2011) reported high (>60\%) and stable micropollutant removal in pilot-scale GAC filters in different sampling campaigns that were 2 years apart, which is a strong indication of biodegradation. Additionally, Bourgin et al. (2018b) reported increased micropollutant removal in time in a GAC filter treating wastewater effluent, which is also compatible with biodegradation and incompatible with removal through adsorption. Additionally, the authors observe that after a 28,000 bed volumes (16 months), removal efficiencies in GAC filters that started up with fresh or pre-loaded GAC are comparable. All these results demonstrate that biodegradation is a very relevant removal mechanism for micropollutants removal in GAC filters used in WWTPs. Furthermore, higher micropollutants removal is observed when the filter influent is previously ozonated, which increases biodegradability of micropollutants and background organic matter, favouring cometabolic biodegradation (Bourgin et al., 2018; Reungoat et al., 2012). High and stable removal efficiencies observed in GAC filters treating wastewater after long periods can only result from microbial degradation from the pollutants in the liquid phase or from a residual adsorption capacity that is maintained due to bioregeneration. These results show that bioregeneration likely takes place in full scale GAC filters, although the extent of its benefits on extending the GAC lifetime is unknown. 


\subsubsection{In situ vs. offline bioregeneration}

Bioregeneration can be stimulated by applying favourable conditions for micropollutants biodegradation. This can be done by supplying carbon sources, electron donors and acceptors or nutrients to the filters and maintaining suitable temperatures. However, increasing microbial activity in GAC filters can reduce the GAC adsorption capacity for several contaminants, due to adsorption of microbial metabolites (Chapter 4 ) and mass transfer limitations in the biofilm (see 6.5.2). The reduced adsorption capacity will have an undesirable impact on the removal of the non-biodegradable fraction of NOM and nonbiodegradable micropollutants. Additionally, biofilm growth has to be controlled, as excessive growth can reduce effluent quality, deplete dissolved oxygen from water and clog the filter increasing the need for backwashing. Increasing micropollutants biodegradation whilst maintaining optimal conditions for GAC filtration in a treatment train is challenging. Quantifying micropollutants biodegradation in the presence of GAC is crucial so that the trade-off between reduced adsorption capacity and increased removal through biodegradation can be evaluated.

In case stimulated biodegradation compromises adsorption to the point that overall micropollutants removal is negatively affected, offline bioregeneration should be considered. Offline bioregeneration consists in applying a separate step for the regeneration of GAC, instead of doing it in situ, i.e., simultaneously with GAC filtration in a treatment train (Figure 6.7). Decoupling the bioregeneration step from the regular filter operation allows more flexible operational conditions in the bioregeneration step. These conditions include longer contact times or batch feeding mode, addition of biodegradable carbon sources, nutrients and oxygen, and higher temperatures. Moreover, in cases when a small number of micropollutants prevail in the filter influent, specific conditions can be steered to these micropollutants. For instance, additional carbon sources might not benefit biodegradation of micropollutants that are metabolically biodegraded. Additionally, some micropollutants are preferably degraded in anoxic conditions, which would avoid the need for aeration. Offline bioregeneration in drinking water treatment also reduces the concern with microbial safety of the effluent since the effluent of the bioregeneration step is not the final product of water treatment. 

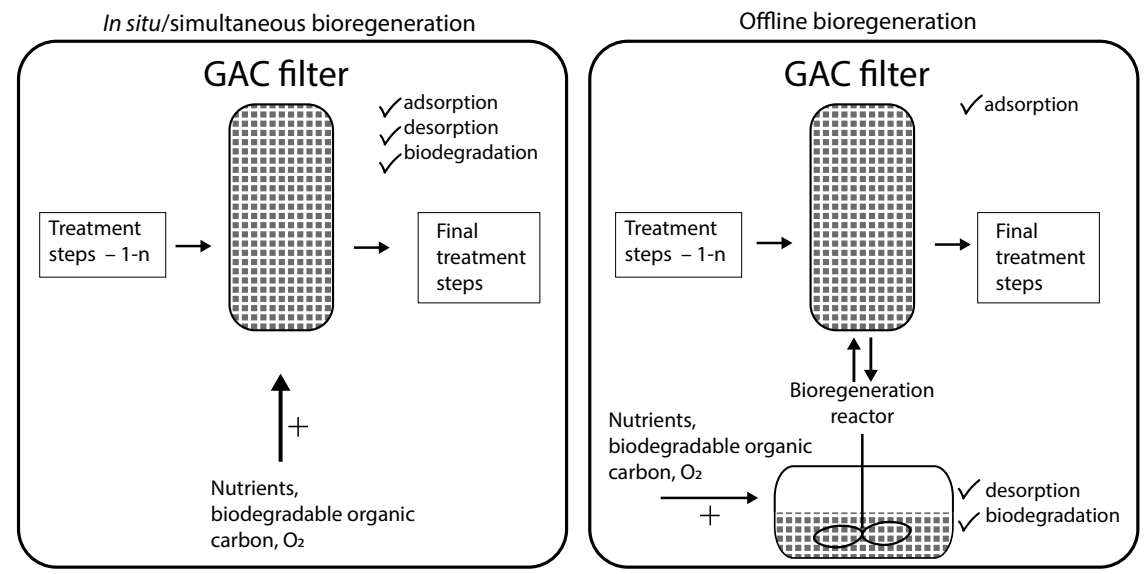

Figure 6.7: In situ vs. offline bioregeneration.

For in situ bioregeneration, microbial activity should be stimulated while maintaining microbial safety and stability of filter effluent. This means balancing suspended and attached growth so that enough microbial activity exists in the biofilm while the effluent quality is not compromised. The backwash regime is an important tool in maintaining a desired level of biomass activity in the filter and removing weakly attached biomass (Flemming, 2020; Gibert et al., 2013). However, a balance needs to be found since high biofilm density caused by elevated shear stress can negatively affect GAC bioregeneration (Abromaitis et al., 2017).

If on the one hand, micropollutants removal through adsorption in drinking water GAC filters benefits from the low concentrations of NOM, on the other hand, micropollutants biodegradation and consequent GAC bioregeneration can be limited by the oligotrophic conditions present in these filters. Additionally, nutrients such as phosphorus might limit biological activity in GAC filters in drinking water treatment (Scholz and Martin, 1997). To promote in situ bioregeneration in GAC filters, microbial activity can be stimulated via the addition of nutrients and biodegradable organic matter. However, these changes might negatively impact the quality of the filtered water. This conflict suggests that offline bioregeneration is more suitable than in situ bioregeneration for GAC filters in drinking water treatment.

\subsection{Conclusion}

This thesis shows that micropollutants biodegradation promotes micropollutants desorption from GAC which leads to regeneration of the GAC adsorptive capacity. Previous research 
shows that biodegradation significantly contributes to removing micropollutants in GAC filters. Furthermore, synergistic effects of adsorption and biodegradation have the potential of promoting biodegradation of slowly degradable micropollutants. Exploring the combination of these two processes can reduce our reliance on energy intensive technologies to reactivate GAC.

Bioregeneration has important limitations, since non-biodegradable adsorbates are not removed and remain on the GAC. Additionally, desorption hysteresis can limit the regeneration of adsorption sites in the narrowest pores. These limitations indicate that bioregeneration most likely will not fully replace current reactivation methods. Nevertheless, biodegradation of biodegradable micropollutants releases adsorption sites increasing also adsorption capacity for recalcitrant compounds (Putz et al., 2005). Desorption hysteresis can be minimized by choosing GAC with a wide pore size distribution, facilitating desorption. Therefore, bioregeneration has a large potential to reduce the frequency in which conventional reactivation methods are applied and extend the lifetime of GAC filters. 


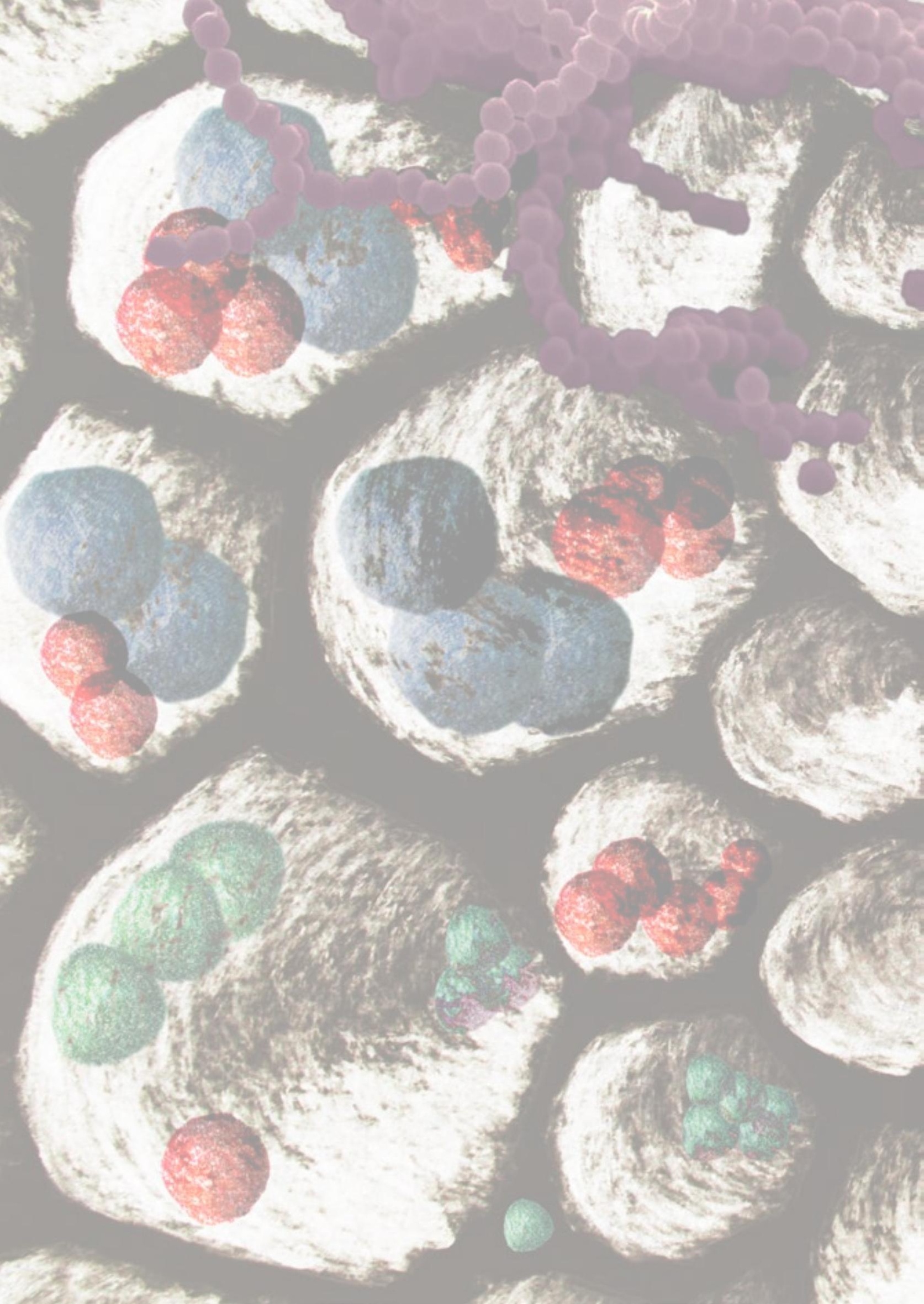


A

Abromaitis, V., Racys, V., van der Marel, P., Meulepas, R.J.W., 2016. Biodegradation of persistent organics can overcome adsorption-desorption hysteresis in biological activated carbon systems. Chemosphere 149, 183-189.

Abromaitis, V., Racys, V., van der Marel, P., Ni, G., Dopson, M., Wolthuizen, A.L., Meulepas, R.J.W., 2017. Effect of shear stress and carbon surface roughness on bioregeneration and performance of suspended versus attached biomass in metoprolol-loaded biological activated carbon systems. Chemical Engineering Journal 317, 503-511.

Ahn, Y., Cho, D., Kabra, A.N., Ji, M., Yoon, Y., 2015. Removal of iopromide and its intermediates from ozone-treated water using granular activated carbon. Water, Air, E Soil Pollution 226, 346.

Aktaş, Ö., Çeçen, F., 2009. Cometabolic bioregeneration of activated carbons loaded with 2chlorophenol. Bioresource Technology 100, 4604-4610.

Aktaş, Ö., Çeçen, F., 2007. Bioregeneration of activated carbon: A review. International Biodeterioration EBiodegradation 59, 257-272.

Aktaş, Ö., Çeçen, F., 2006a. Effect of activation type on bioregeneration of various activated carbons loaded with phenol. Journal of Chemical Technology and Biotechnology 81, 10811092.

Aktaş, Ö., Çeçen, F., 2006b. Effect of type of carbon activation on adsorption and its reversibility. Journal of Chemical Technology and Biotechnology 81, 94-101.

Al-Degs, Y.S., El-Barghouthi, M.I., El-Sheikh, A.H., Walker, G.M., 2008. Effect of solution pH, ionic strength, and temperature on adsorption behavior of reactive dyes on activated carbon. Dyes and Pigments 77, 16-23.

Albers, C.N., Feld, L., Ellegaard-Jensen, L., Aamand, J., 2015. Degradation of trace concentrations of the persistent groundwater pollutant 2,6-dichlorobenzamide (BAM) in bioaugmented rapid sand filters. Water Research 83, 61-70.

Alidina, M., Li, D., Ouf, M., Drewes, J.E., 2014. Role of primary substrate composition and concentration on attenuation of trace organic chemicals in managed aquifer recharge systems. Journal of Environmental Management 144, 58-66.

Alotaibi, M.D., McKinley, A.J., Patterson, B.M., Reeder, A.Y., 2015. Benzotriazoles in the aquatic environment: a review of their occurrence, toxicity, degradation and analysis. Water, Air, $\mathcal{E}$ Soil Pollution 226, 226.

Altmann, J., Rehfeld, D., Träder, K., Sperlich, A., Jekel, M., 2016. Combination of granular activated carbon adsorption and deep-bed filtration as a single advanced wastewater treatment step for organic micropollutant and phosphorus removal. Water Research 92, 131-139.

Alupoaei, C.E., Olivares, J.A., García-Rubio, L.H., 2004. Quantitative spectroscopy analysis of prokaryotic cells: Vegetative cells and spores. Biosensors \& Bioelectronics 19, 893-903. 
Alvarino, T., Suarez, S., Lema, J.M., Omil, F., 2014. Understanding the removal mechanisms of PPCPs and the influence of main technological parameters in anaerobic UASB and aerobic CAS reactors. Journal of Hazardous Materials 278, 506-513.

Alves, T.C., Cabrera-Codony, A., Barceló, D., Rodriguez-Mozaz, S., Pinheiro, A., GonzalezOlmos, R., 2018. Influencing factors on the removal of pharmaceuticals from water with micro-grain activated carbon. Water Research 144, 402-412.

An, H., Li, X., Yang, Q., Wang, D., Xie, T., Zhao, J., Xu, Q., Chen, F., Zhong, Y., Yuan, Y., Zeng, G., 2017. The behavior of melamine in biological wastewater treatment system. Journal of Hazardous Materials 322, 445-453.

Aronson, D., Boethling, R., Howard, P., Stiteler, W., 2006. Estimating biodegradation half-lives for use in chemical screening. Chemosphere 63, 1953-1960.

Aschermann, G., Neubert, L., Zietzschmann, F., Jekel, M., 2019a. Impact of different DOM size fractions on the desorption of organic micropollutants from activated carbon. Water Research 161, 161-170.

Aschermann, G., Schröder, C., Zietzschmann, F., Jekel, M., 2019b. Organic micropollutant desorption in various water matrices - Activated carbon pore characteristics determine the reversibility of adsorption. Chemosphere 237, 124415.

Aschermann, G., Zietzschmann, F., Jekel, M., 2018. Influence of dissolved organic matter and activated carbon pore characteristics on organic micropollutant desorption. Water Research $133,123-131$.

\section{B}

Baken, K.A., Sjerps, R.M.A., Schriks, M., van Wezel, A.P., 2018. Toxicological risk assessment and prioritization of drinking water relevant contaminants of emerging concern. Environment International 118, 293-303.

Batt, A.L., Kim, S., Aga, D.S., 2006. Enhanced biodegradation of iopromide and trimethoprim in nitrifying activated sludge. Environmental Science \& Technology 40, 7367-7373.

Bäuerlein, P.S., Mansell, J.E., Ter Laak, T.L., De Voogt, P., 2012. Sorption behavior of charged and neutral polar organic compounds on solid phase extraction materials: Which functional group governs sorption? Environmental Science \& Technology 46, 954-961.

Bayer, P., Heuer, E., Karl, U., Finkel, M., 2005. Economical and ecological comparison of granular activated carbon (GAC) adsorber refill strategies. Water Research 39, 1719-1728.

Benner, J., Helbling, D.E., Kohler, H.E., Wittebol, J., Kaiser, E., Prasse, C., Ternes, T.A., Albers, C.N., Aamand, J., Horemans, B., Springael, D., Walravens, E., Boon, N., 2013. Is biological treatment a viable alternative for micropollutant removal in drinking water treatment processes? Water Research 47, 5955-5976.

Benstoem, F., Nahrstedt, A., Boehler, M., Knopp, G., Montag, D., Siegrist, H., Pinnekamp, J., 2017. Performance of granular activated carbon to remove micropollutants from municipal wastewater-A meta-analysis of pilot- and large-scale studies. Chemosphere $185,105-118$. 
Bertelkamp, C., Hofman-Caris, R., Siegers, W., Beerendonk, E.F., Nugteren, S., 2017. Biodegradation of pyrazole in rapid sand filters., in: Conf. Proc. 10th Micropol Ecohazard Conf. IWA. pp. 17-20.

Besha, A.T., Gebreyohannes, A.Y., Tufa, R.A., Bekele, D.N., Curcio, E., Giorno, L., 2017. Removal of emerging micropollutants by activated sludge process and membrane bioreactors and the effects of micropollutants on membrane fouling: A review. Journal of Environmental Chemical Engineering 5, 2395-2414.

Bexfield, L.M., Toccalino, P.L., Belitz, K., Foreman, W.T., Furlong, E.T., 2019. Hormones and pharmaceuticals in groundwater used as a source of drinking water across the United States. Environmental Science \& Technology 53, 2950-2960.

Bhadra, B.N., Jhung, S.H., 2017. A remarkable adsorbent for removal of contaminants of emerging concern from water: porous carbon derived from metal azolate framework- 6 . Journal of Hazardous Materials 340, 179-188.

Blair, B., Nikolaus, A., Hedman, C., Klaper, R., Grundl, T., 2015. Evaluating the degradation, sorption, and negative mass balances of pharmaceuticals and personal care products during wastewater treatment. Chemosphere 134, 395-401.

Blanchard, G., Maunaye, M., Martin, G., 1984. Removal of heavy metals from waters by means of natural zeolites. Water Research 18, 1501-1507.

Boonnorat, J., Techkarnjanaruk, S., Honda, R., Ghimire, A., Angthong, S., Rojviroon, T., Phanwilai, S., 2018. Enhanced micropollutant biodegradation and assessment of nitrous oxide concentration reduction in wastewater treated by acclimatized sludge bioaugmentation. Science of the Total Environment 637-638, 771-779.

Bourgin, M., Beck, B., Boehler, M., Borowska, E., Fleiner, J., Salhi, E., Teichler, R., von Gunten, U., Siegrist, H., McArdell, C.S., 2018. Evaluation of a full-scale wastewater treatment plant upgraded with ozonation and biological post-treatments: Abatement of micropollutants, formation of transformation products and oxidation by-products. Water Research 129, 486498.

Bourneuf, S., Jacob, M., Albasi, C., Sochard, S., Richard, R., Manero, M.H., 2016. Desorption experiments and modeling of micropollutants on activated carbon in water phase: application to transient concentrations mitigation. International Journal of Environmental Science and Technology 13, 1-10.

Briones, R.M., Sarmah, A.K., 2018. Detailed sorption characteristics of the anti-diabetic drug metformin and its transformation product guanylurea in agricultural soils. Science of the Total Environment 630, 1258-1268.

Briones, R.M., Zhuang, W., Sarmah, A.K., 2018. Biodegradation of metformin and guanylurea by aerobic cultures enriched from sludge. Environmental Pollution 243, 255-262.

Brunner, A.M., Bertelkamp, C., Dingemans, M.M.L., Kolkman, A., Wols, B., Harmsen, D., Siegers, W., Martijn, B.J., Oorthuizen, W.A., ter Laak, T.L., 2020. Integration of target analyses, non-target screening and effect-based monitoring to assess OMP related water quality changes in drinking water treatment. Science of the Total Environment 705, 135779. 
Brunner, A.M., Dingemans, M.M.L., Baken, K.A., van Wezel, A.P., 2019. Prioritizing anthropogenic chemicals in drinking water and sources through combined use of mass spectrometry and ToxCast toxicity data. Journal of Hazardous Materials 364, 332-338.

Buchanan, W., Roddick, F., Porter, N., Drikas, M., 2004. Enhanced biodegradability of UV and VUV pre-treated natural organic matter. Water Science \& Technology: Water Supply 4, 103111.

Butkovskyi, A., Sevenou, L., Meulepas, R.J.W., Hernandez Leal, L., Zeeman, G., Rijnaarts, H.H.M., 2018. Micropollutant removal from black water and grey water sludge in a UASB-GAC reactor. Water Science \& Technology 77, 1137-1148.

Buttiglieri, G., Peschka, M., Frömel, T., Müller, J., Malpei, F., Seel, P., Knepper, T.P., 2009. Environmental occurrence and degradation of the herbicide n-chloridazon. Water Research $43,2865-2873$.

\section{C}

Çeçen, F., Aktaş, Ö., 2012. Activated carbon for water and wastewater treatment: integration of adsorption and biological treatment. WILEY-VCH Verlag GmbH \& Co. KGaA, Weinheim.

Cha, W., Choi, H., Ha, S., 1998. The buffer effect of biological activated carbon to the shock loadings in sequencing batch reactor. Environmental Engineering Research 3, 41-46.

Chan, P.-Y., Lim, P.-E., Ng, S.-L., Seng, C.-E., 2018. Bioregeneration of granular activated carbon loaded with phenolic compounds: effects of biological and physico-chemical factors. International Journal of Environmental Science and Technology 15, 1699-1712.

Chaumet, B., Morin, S., Boutry, S., Mazzella, N., 2019. Diuron sorption isotherms in freshwater biofilms. Science of the Total Environment 651, 1219-1225.

Chaves, M. de J.S., Barbosa, S.C., Mallinowski, M. de M., Volpato, D., Castro, I.B., Franco, T.C.R. dos S.F., Primel, E.G., 2020. Pharmaceuticals and personal care products in a Brazilian wetland of international importance: Occurrence and environmental risk assessment. Science of The Total Environment 734, 139374.

Chen, F., Peldszus, S., Elhadidy, A.M., Legge, R.L., Van Dyke, M.I., Huck, P.M., 2016. Kinetics of natural organic matter (NOM) removal during drinking water biofiltration using different NOM characterization approaches. Water Research 104, 361-370.

Christoffels, E., Brunsch, A., Wunderlich-Pfeiffer, J., Mertens, F.M., 2016. Monitoring micropollutants in the Swist river basin. Water Science \& Technology 74, 2280-2296.

Cid-Cerón, M.M., Guzmán-Hernández, D.S., Ramírez-Silva, M.T., Galano, A., Romero-Romo, M., Palomar-Pardavé, M., 2016. New insights on the kinetics and mechanism of the electrochemical oxidation of diclofenac in neutral aqueous medium. Electrochimica Acta 199, 92-98.

Cirja, M., Ivashechkin, P., Schäffer, A., Corvini, P.F.X., 2008. Factors affecting the removal of organic micropollutants from wastewater in conventional treatment plants (CTP) and membrane bioreactors (MBR). Reviews in Environmental Science and Biotechnology 7, 61-78. 
Cook, A.M., Huetter, R., 1981. s-Triazines as nitrogen sources for bacteria. Journal of Agriculture and Food Chemistry 29, 1135-1143.

Corwin, C.J., Summers, R.S., 2011. Adsorption and desorption of trace organic contaminants from granular activated carbon adsorbers after intermittent loading and throughout backwash cycles. Water Research 45, 417-426.

Criddle, C.S., 1993. The kinetics of cometabolism. Biotechnology and Bioengineering 42, 674-674.

\section{D}

Dab̧rowski, A., Podkościelny, P., Hubicki, Z., Barczak, M., 2005. Adsorption of phenolic compounds by activated carbon - A critical review. Chemosphere 58, 1049-1070.

Dalahmeh, S., Björnberg, E., Elenström, A.K., Niwagaba, C.B., Komakech, A.J., 2020. Pharmaceutical pollution of water resources in Nakivubo wetlands and Lake Victoria, Kampala, Uganda. Science of the Total Environment 710, 136347.

Dalton, H., Stirling, D.I., 1982. Co-metabolism. Philosophical Transactions of the Royal Society London 297, 481-496.

Dawas-Massalha, A., Gur-Reznik, S., Lerman, S., Sabbah, I., Dosoretz, C.G., 2014. Co-metabolic oxidation of pharmaceutical compounds by a nitrifying bacterial enrichment. Bioresource Technology 167, 336-342.

de Duve, C., 1996. The birth of complex cells. Scientific American 274, 50-57.

de Jong, C.Y., van Nieuwenhuijzen, A.F., Dekker, A., Liu, T.K., de Smet, S.J.S., 2018. STOWA 2018-67 Proof of concept en laboratorium- onderzoek verwijdering microverontreinigingen uit rwzi -effluent met het O3-Step ${ }^{\circledR}$ Filter.

de Jonge, R.J., Breure, A.M., Van Andel, J.G., 1996. Bioregeneration of powdered activated carbon (PAC) loaded with aromatic compound. Water Research 30, 875-882.

de Lange, H.J., Noordoven, W., Murk, A.J., Lürling, M., Peeters, E.T.H.M., 2006. Behavioural responses of Gammarus pulex (Crustacea, Amphipoda) to low concentrations of pharmaceuticals. Aquatic Toxicology 78, 209-216.

de Ridder, D.J., Verliefde, A.R.D., Heijman, S.G.J., Verberk, J.Q.J.C., Rietveld, L.C., Van Der Aa, L.T.J., Amy, G.L., Van Dijk, J.C., 2011. Influence of natural organic matter on equilibrium adsorption of neutral and charged pharmaceuticals onto activated carbon. Water Science $\mathcal{E}$ Technology 63, 416-423.

de Ridder, D.J., Verliefde, A.R.D., Schoutteten, K., Van Der Linden, B., Heijman, S.G.J., Beurroies, I., Denoyel, R., Amy, G.L., Van Dijk, J.C., 2013. Relation between interfacial energy and adsorption of organic micropollutants onto activated carbon. Carbon 53, 153160.

de Ridder, D.J., Villacorte, L., Verliefde, A.R.D., Verberk, J.Q.J.C., Heijman, S.G.J., Amy, G.L., van Dijk, J.C., 2010. Modeling equilibrium adsorption of organic micropollutants onto activated carbon. Water Research 44, 3077-3086.

de Wit, R., Bouvier, T., 2006. "Everything is everywhere, but, the environment selects"; what 
did Baas Becking and Beijerinck really say? Environmental Microbiology 8, 755-758.

Ding, H., Li, Y., Hou, J., Wang, Q., Wu, Y., 2014. Sorption behavior and modeling of endocrinedisrupting chemicals on natural sediments: role of biofilm covered on surface. Environmental Science and Pollution Research 22, 1380-1388.

Dittmar, S., Zietzschmann, F., Mai, M., Worch, E., Jekel, M., Ruhl, A.S., 2018. Simulating effluent organic matter competition in micro-pollutant adsorption onto activated carbon using a surrogate competitor. Environmental Science \& Technology 52, 7859-7866.

Dubey, S.P., Dwivedi, A.D., Sillanpää, M., Gopal, K., 2010. Artemisia vulgaris-derived mesoporous honeycomb-shaped activated carbon for ibuprofen adsorption. Chemical Engineering Journal 165, 537-544.

E

Ehrl, B.N., Kundu, K., Gharasoo, M., Marozava, S., Elsner, M., 2019. Rate-limiting mass transfer in micropollutant degradation revealed by isotope fractionation in chemostat. Environmental Science \& Technology 53, 1197-1205.

El-Sayed, W.S., El-Baz, A.F., Othman, A.M., 2006. Biodegradation of melamine formaldehyde by Micrococcus sp. strain MF-1 isolated from aminoplastic wastewater effluent. International Biodeterioration E Biodegradation 57, 75-81.

Elizalde-Velázquez, G.A., Gómez-Oliván, L.M., 2020. Occurrence, toxic effects and removal of metformin in the aquatic environments in the world: Recent trends and perspectives. Science of the Total Environment 702.

Etteieb, S., Tarhouni, J., Isoda, H., 2019. Cellular stress response biomarkers for toxicity potential assessment of treated wastewater complex mixtures. Water and Environment Journal 33, 4-13.

$\mathbf{F}$

Falås, P., Longrée, P., La Cour Jansen, J., Siegrist, H., Hollender, J., Joss, A., 2013. Micropollutant removal by attached and suspended growth in a hybrid biofilm-activated sludge process. Water Research 47, 4498-4506.

Falås, P., Wick, A., Castronovo, S., Habermacher, J., Ternes, T.A., Joss, A., 2016. Tracing the limits of organic micropollutant removal in biological wastewater treatment. Water Research 95, 240-249.

Fernandez-Fontaina, E., Carballa, M., Omil, F., Lema, J.M., 2014. Modelling cometabolic biotransformation of organic micropollutants in nitrifying reactors. Water Research 65, 371-383.

Fischer, A., van Wezel, A.P., Hollender, J., Cornelissen, E., Hofman, R., van der Hoek, J.P., 2019. Development and application of relevance and reliability criteria for water treatment removal efficiencies of chemicals of emerging concern. Water Research 161, 274-287.

Fischer, K., Majewsky, M., 2014. Cometabolic degradation of organic wastewater micropollutants by activated sludge and sludge-inherent microorganisms. Applied 
Microbiology and Biotechnology 98, 6583-6597.

Flemming, H.C., 2020. Biofouling and me: My Stockholm syndrome with biofilms. Water Research 173, 115576.

Flemming, H.C., 1995. Sorption sites in biofilms. Water Science E Technology 32, 93.

G

Galíndez-Nájera, S.P., Llamas-Martínez, M.A., Ruiz-Ordaz, N., Juárez-Ramírez, C., MondragónParada, M.E., Ahuatzi-Chacón, D., Galíndez-Mayer, J., 2009. Cyanuric acid biodegradation by a mixed bacterial culture of Agrobacterium tumefaciens and Acinetobacter sp. in a packed bed biofilm reactor. Journal of Industrial Microbiology E Biotechnology 36, 275-284.

Ganguly, S., Jacob, S.K., 2017. Therapeutic outlook of pyrazole analogs: a mini review. MiniReviews in Medicinal Chemistry 17, 959-983.

Gibert, O., Lefèvre, B., Fernández, M., Bernat, X., Paraira, M., Calderer, M., Martínez-Lladó, X., 2013. Characterising biofilm development on granular activated carbon used for drinking water production. Water Research 47, 1101-1110.

Gonzalez-Gil, L., Carballa, M., Lema, J.M., 2017. Cometabolic enzymatic transformation of organic micropollutants under methanogenic conditions. Environmental Science $\mathcal{E}$ Technology 51, 2963-2971.

Grandclément, C., Seyssiecq, I., Piram, A., Wong-Wah-Chung, P., Vanot, G., Tiliacos, N., Roche, N., Doumenq, P., 2017. From the conventional biological wastewater treatment to hybrid processes, the evaluation of organic micropollutant removal: A review. Water Research 111, 297-317.

Greenstein, K.E., Lew, J., Dickenson, E.R. V, Wert, E.C., 2018. Investigation of biotransformation, sorption, and desorption of multiple chemical contaminants in pilot-scale drinking water biofilters. Chemosphere 200, 248-256.

Guillossou, R., Le Roux, J., Mailler, R., Vulliet, E., Morlay, C., Nauleau, F., Gasperi, J., Rocher, V., 2019. Organic micropollutants in a large wastewater treatment plant: What are the benefits of an advanced treatment by activated carbon adsorption in comparison to conventional treatment? Chemosphere 218, 1050-1060.

Gunten, U. von, 2018. Oxidation processes in water treatment: are we on track? Environmental Science \& Technology 52, 5062-5075.

Gupta, V.K., Gupta, B., Rastogi, A., Agarwal, S., Nayak, A., 2011. Pesticides removal from waste water by activated carbon prepared from waste rubber tire. Water Research 45, 4047-4055.

\section{$\mathbf{H}$}

Han, L., Liu, W., Chen, M., Zhang, M., Liu, S., Sun, R., Fei, X., 2013. Comparison of NOM removal and microbial properties in up-flow/down-flow BAC filter. Water Research 47, 4861-4868.

Han, N., Reinhard, M., Khan, E., Chen, H., Tung, V., Li, Y., Giek, S., Nguyen, Q.B., Saeidi, N., 
Gin, K.Y., 2019. Emerging contaminants in wastewater, stormwater runoff, and surface water: Application as chemical markers for diffuse sources. Science of the Total Environment 676, 252-267.

Hart, D.S., Davis, L.C., Erickson, L.E., Callender, T.M., 2004. Sorption and partitioning parameters of benzotriazole compounds. Microchemical Journal 77, 9-17.

He, H.-Q., Lie, X.-H., Jian-QuanWeng, Tan, C.-X., 2017. Synthesis and herbicidal activity of some novel pyrazole derivatives. Letters in Drug Design E Discovery 4, 195-200.

Hellauer, K., Martínez Mayerlen, S., Drewes, J.E., Hübner, U., 2019. Biotransformation of trace organic chemicals in the presence of highly refractory dissolved organic carbon. Chemosphere 215, 33-39.

Herrero, M., Stuckey, D.C., 2015. Bioaugmentation and its application in wastewater treatment: A review. Chemosphere 140, 119-128.

Herzberg, M., Dosoretz, C.G., Green, M., 2005. Increased biofilm activity in BGAC reactors. AIChE Journal 51, 1042-1047.

Herzberg, M., Dosoretz, C.G., Kuhn, J., Klein, S., Green, M., 2006. Visualization of active biomass distribution in a BGAC fluidized bed reactor using GFP tagged Pseudomonas putida F1. Water Research 40, 2704-2712.

Herzberg, M., Dosoretz, C.G., Tarre, S., Green, M., 2003. Patchy biofilm coverage can explain the potential advantage of BGAC reactors. Environmental Science E Technology 37, 4274-4280.

Herzog, B., Lemmer, H., Huber, B., Horn, H., Müller, E., 2014. Xenobiotic benzotriazolesbiodegradation under meso- and oligotrophic conditions as well as denitrifying, sulfatereducing, and anaerobic conditions. Environmental Science and Pollution Research 21, 27952804 .

Hindarso, H., Ismadji, S., Wicaksana, F., Indraswati, N., 2001. Adsorption of benzene and toluene from aqueous solution onto granular activated carbon. Journal of Chemical Engineering Data 46, 788-791.

Ho, Y.S., McKay, G., 1998. Kinetic models for the sorption of dye from aqueous solution by wood. Process Safety and Environmental Protection 76, 183-191.

Hofman-Caris, R., ter Laak, T., Huiting, H., Tolkamp, H., de Man, A., van Diepenbeek, P., Hofman, J., 2019. Origin, fate and control of pharmaceuticals in the urban water cycle: A case study. Water (Switzerland) 11, 1-16.

Hu, J., Shang, R., Heijman, B., Rietveld, L., 2016. Influence of activated carbon preloading by EfOM fractions from treated wastewater on adsorption of pharmaceutically active compounds. Chemosphere 150, 49-56.

Huerta-Fontela, M., Galceran, M.T., Ventura, F., 2011. Occurrence and removal of pharmaceuticals and hormones through drinking water treatment. Water Research 45, 1432-1442.

Hung, H., Lin, T., 2006. Adsorption of MTBE from contaminated water by carbonaceous resins 
and mordenite zeolite. Journal of Hazardous Materials 135, 210-217.

Huntscha, S., Singer, H.P., McArdell, C.S., Frank, C.E., Hollender, J., 2012. Multiresidue analysis of 88 polar organic micropollutants in ground, surface and wastewater using online mixed-bed multilayer solid-phase extraction coupled to high performance liquid chromatography-tandem mass spectrometry. Journal of Chromatography A 1268, 74-83.

\section{I}

Iasur-Kruh, L., Hadar, Y., Minz, D., 2011. Isolation and bioaugmentation of an estradioldegrading bacterium and its integration into a mature biofilm. Applied and Environmental Microbiology 77, 3734-3740.

\section{J}

Jia, Y., Zhang, H., Khanal, S.K., Yin, L., Lu, H., 2019. Insights into pharmaceuticals removal in an anaerobic sulfate-reducing bacteria sludge system. Water Research 161, 191-201.

Jones, L.R., Owen, S.A., Horrell, P., Burns, R.G., 1998. Bacterial inoculation of granular activated carbon filters for the removal of atrazine from surface water. Water Research 32, 2542-2549.

Jones, O.A.H., Voulvoulis, N., Lester, J.N., 2004. Potential ecological and human health risks associated with the presence of pharmaceutically active compounds in the aquatic environment. Critical Reviews in Toxicology 34, 335-350.

Jönsson, K., Aspichueta, E., Sota, A. De, Jansen, J.C., 2001. Evaluation of nitrification-inhibition measurements. Water Science E Technology 43, 201-208.

Joss, A., Zabczynski, S., Göbel, A., Hoffmann, B., Löffler, D., McArdell, C.S., Ternes, T.A., Thomsen, A., Siegrist, H., 2006. Biological degradation of pharmaceuticals in municipal wastewater treatment: Proposing a classification scheme. Water Research 40, 1686-1696.

Jourdin, L., Raes, S.M.T., Buisman, C.J.N., Strik, D.P.B.T.B., 2018. Critical biofilm growth throughout unmodified carbon felts allows continuous bioelectrochemical chain elongation from $\mathrm{CO} 2$ up to caproate at high current density. Frontiers in Energy Research 6, $1-15$.

Jutzi, K., Cook, A.M., Hutter, R., 1982. The degradative pathway of the s-triazine melamine. The steps to ring cleavage. Biochemistry 208, 679-684.

\section{K}

Karanfil, T., Kilduff, J.E., 1999. Role of granular activated carbon surface chemistry on the adsorption of organic compounds. 1. Priority pollutants. Environmental Science $\mathcal{E}$ Technology 33, 3217-3224.

Katsigiannis, A., Noutsopoulos, C., Mantziaras, J., Gioldasi, M., 2015. Removal of emerging pollutants through Granular Activated Carbon. Chemical Engineering Journal 280, 49-57.

Kennedy, A.M., Reinert, A.M., Knappe, D.R.U., Ferrer, I., Summers, R.S., 2015. Full- and pilotscale GAC adsorption of organic micropollutants. Water Research 68, 238-248.

Kennedy, A.M., Summers, R.S., 2015. Effect of DOM size on organic micropollutant adsorption 
by GAC. Environmental Science \& Technology 49, 6617-6624.

Kew, S.L., Adnan, R., Lim, P.E., Seng, C.E., 2016. Bioregeneration of cresol-loaded granular activated carbon using immobilized biomass: Effects of operational factors and chemical structure of cresol isomers. Journal of the Taiwan Institute of Chemical Engineers 63, 386-395.

Kiss, A., Fries, E., 2009. Occurrence of benzotriazoles in the rivers Main, Hengstbach, and Hegbach (Germany). Environmental Science and Pollution Research 16, 702-710.

Klimenko, N., Smolin, S., Grechanyk, S., Kofanov, V., Nevynna, L., Samoylenko, L., 2003. Bioregeneration of activated carbons by bacterial degraders after adsorption of surfactants from aqueous solutions. Colloids and Surfaces A: Physicochemical and Engineering Aspects 230, 141-158.

Klimenko, N.A., Polyakova, T. V., Savchina, L.A., 2010. Influence of sodium azide on the removal of fulvic acids by activated carbon. Journal of Water Chemistry and Technology 32, 329-335.

Knezev, A., 2015. Microbial activity in granular activated carbon filters in drinking water treatment. Wageningen University.

Knopp, G., Prasse, C., Ternes, T.A., Cornel, P., 2016. Elimination of micropollutants and transformation products from a wastewater treatment plant effluent through pilot scale ozonation followed by various activated carbon and biological filters. Water Research 100 , 580-592.

Kools, S., Loon, A. van, Sjerps, R., Rosenthal, L., 2019. De kwaliteit van bronnen van drinkwater in Nederland. KWR 2019.072 I Augustus 2019.

Kormos, J.L., Schulz, M., Kohler, H.P.E., Ternes, T.A., 2010. Biotransformation of selected iodinated X-ray contrast media and characterization of microbial transformation pathways. Environmental Science E Technology 44, 4998-5007.

Kosjek, T., Heath, E., Pérez, S., Petrovic, M., 2009. Metabolism studies of diclofenac and clofibric acid in activated sludge bioreactors using liquid chromatography with quadrupole time-of-flight mass spectrometry. Journal of Hydrology 372, 109-117.

Kovalova, L., Knappe, D.R.U., Lehnberg, K., Kazner, C., Hollender, J., 2013. Removal of highly polar micropollutants from wastewater by powdered activated carbon. Environmental Science and Pollution Research 20,3607-3615.

Kumerer, K., 2010. Pharmaceuticals in the environment. Annual Review of Environment and Resources 35, 57-75.

Kumwimba, M.N., Meng, F., 2019. Roles of ammonia-oxidizing bacteria in improving metabolism and cometabolism of trace organic chemicals in biological wastewater treatment processes: A review. Science of the Total Environment 659, 419-441.

Kyriakopoulos, G., Doulia, D., 2006. Adsorption of pesticides on carbonaceous and polymeric materials from aqueous solutions: a review. Separation $\mathcal{E}$ Purification Reviews 35, 97-191. 
L

Lacerda, V. da S., López-Sotelo, J.B., Correa-Guimarães, A., Hernández-Navarro, S., SánchezBáscones, M., Navas-Gracia, L.M., Martín-Ramos, P., Martín-Gil, J., 2015. Rhodamine B removal with activated carbons obtained from lignocellulosic waste. Journal of Environmental Management 155, 67-76.

Langenhoff, A., Inderfurth, N., Veuskens, T., Schraa, G., Blokland, M., Kujawa-Roeleveld, K., Rijnaarts, H., 2013. Microbial removal of the pharmaceutical compounds ibuprofen and diclofenac from wastewater. BioMed Research International 2013, 325806.

Langmuir, I., 1918. The adsorption of gases on plane surfaces of glass, mica and platinum. Journal of the American Chemical Society 40, 1361-1403.

Largitte, L., Pasquier, R., 2016. A review of the kinetics adsorption models and their application to the adsorption of lead by an activated carbon. Chemical Engineering Research and Design 109, 495-504.

Launay, M.A., Dittmer, U., Steinmetz, H., 2016. Organic micropollutants discharged by combined sewer overflows - Characterisation of pollutant sources and stormwater-related processes. Water Research 104, 82-92.

Lautenschlager, K., Hwang, C., Ling, F., Liu, W.T., Boon, N., Köster, O., Egli, T., Hammes, F., 2014. Abundance and composition of indigenous bacterial communities in a multi-step biofiltration-based drinking water treatment plant. Water Research 62, 40-52.

Lee, V.K.C., Mckay, G., 2004. Comparison of solutions for the homogeneous surface diffusion model applied to adsorption systems. Chemical Engineering Journal 98, 255-264.

Lehtola, M.J., Miettinen, I.T., Vartiainen, T., Myllykangas, T., Martikainen, P.J., 2001. Microbially available organic carbon, phosphorus, and microbial growth in ozonated drinking water. Water Research 35, 1635-1640.

Lengeler, J.W., Drews, G., Schlegel, H.G., 1999. Biology of the Prokaryotes. Wiley, Stuttgart.

Lesage, G., Sperandio, M., Tiruta-Barna, L., 2010. Analysis and modelling of non-equilibrium sorption of aromatic micro-pollutants on GAC with a multi-compartment dynamic model. Chemical Engineering Journal 160, 457-465.

Li, C., Moe, W.M., 2005. Activated carbon load equalization of discontinuously generated acetone and toluene mixtures treated by biofiltration. Environmental Science \& Technology 39, 2349-2356.

Li, L., Quinlivan, P.A., Knappe, D.R.U., 2002. Effects of activated carbon surface chemistry and pore structure on the adsorption of organic contaminants from aqueous solution. Carbon 40, 2085-2100.

Li, Q., Snoeyink, V.L., Mariãas, B.J., Campos, C., 2003a. Elucidating competitive adsorption mechanisms of atrazine and NOM using model compounds. Water Research 37, 773-784.

Li, Q., Snoeyink, V.L., Mariñas, B.J., Campos, C., 2003b. Pore blockage effect of NOM on atrazine adsorption kinetics of PAC: The roles of PAC pore size distribution and NOM 
molecular weight. Water Research 37, 4863-4872.

Lima, L., Baêta, B.E.L., Lima, D.R.S., Afonso, R.J.C.F., Aquino, S.F. De, Libânio, M., 2015. Comparison between two forms of granular activated carbon for the removal of pharmaceuticals from different waters. Environmental Technology 3330, 1-34.

Liu, G., Zheng, S., Yin, D., Xu, Z., Fan, J., Jiang, F., 2006. Adsorption of aqueous alkylphenol ethoxylate surfactants by mesoporous carbon CMK-3. Journal of Colloid and Interface Science 302, 47-53.

Loos, R., Gawlik, B.M., Locoro, G., Rimaviciute, E., Contini, S., Bidoglio, G., 2009. EU-wide survey of polar organic persistent pollutants in European river waters. Environmental Pollution 157, 561-568.

Loos, R., Locoro, G., Comero, S., Contini, S., Schwesig, D., Werres, F., Balsaa, P., Gans, O., Weiss, S., Blaha, L., Bolchi, M., Gawlik, B.M., 2010. Pan-European survey on the occurrence of selected polar organic persistent pollutants in ground water. Water Research 44, 4115-4126.

López-Ramón, M.V., Ocampo-Pérez, R., Bautista-Toledo, M.I., Rivera-Utrilla, J., MorenoCastilla, C., Sánchez-Polo, M., 2019. Removal of bisphenols A and S by adsorption on activated carbon clothes enhanced by the presence of bacteria. Science of the Total Environment 669, 767-776.

Lu, Q., Sorial, G.A., 2004. The role of adsorbent pore size distribution in multicomponent adsorption on activated carbon. Carbon 42, 3133-3142.

Luo, Y., Guo, W., Ngo, H.H., Nghiem, L.D., Hai, F.I., Zhang, J., Liang, S., Wang, X.C., 2014. A review on the occurrence of micropollutants in the aquatic environment and their fate and removal during wastewater treatment. Science of the Total Environment 473-474, 619641.

\section{$\mathbf{M}$}

Ma, B., Arnold, W.A., Hozalski, R.M., 2018. The relative roles of sorption and biodegradation in the removal of contaminants of emerging concern (CECs) in GAC-sand biofilters. Water Research 146, 67-76.

Mailler, R., Gasperi, J., Coquet, Y., Derome, C., Buleté, A., Vulliet, E., Bressy, A., Varrault, G., Chebbo, G., Rocher, V., 2016. Removal of emerging micropollutants from wastewater by activated carbon adsorption: Experimental study of different activated carbons and factors influencing the adsorption of micropollutants in wastewater. Journal of Environmental Chemical Engineering 4, 1102-1109.

Marcantonio, C., Bertelkamp, C., Bel, N. Van, Pronk, T.E., Peer, H.A., Wielen, P. Van Der, Brunner, A.M., 2020. Organic micropollutant removal in full-scale rapid sand filters used for drinking water treatment in The Netherlands and Belgium. Chemosphere 260, 127630.

Margot, J., Kienle, C., Magnet, A., Weil, M., Rossi, L., de Alencastro, L.F., Abegglen, C., Thonney, D., Chèvre, N., Schärer, M., Barry, D.A., 2013. Treatment of micropollutants in municipal wastewater: Ozone or powdered activated carbon? Science of the Total Environment 461-462, 480-498. 
Markiewicz, M., Jungnickel, C., Stolte, S., Białk-Bielińska, A., Kumirska, J., Mrozik, W., 2017a. Ultimate biodegradability and ecotoxicity of orally administered antidiabetic drugs. Journal of Hazardous Materials 333, 154-161.

Markiewicz, M., Jungnickel, C., Stolte, S., Białk-Bielińska, A., Kumirska, J., Mrozik, W., 2017b. Primary degradation of antidiabetic drugs. Journal of Hazardous Materials 324, 428-435.

Marsh, H., Rodriguez-Reinoso, F., 2006. Activated Carbon. Elsevier Science Ltd.

Martin, G., Richard, Y., 1982. Elements of the inhibition of nitrifying bacteria. Water Science $\mathcal{E}$ Technology 14, 781-794.

Martínez-Alcalá, I., Pellicer-Martínez, F., Fernández-López, C., 2018. Pharmaceutical grey water footprint: Accounting, influence of wastewater treatment plants and implications of the reuse. Water Research 135, 278-287.

Masson, S., Gineys, M., Delpeux-Ouldriane, S., Reinert, L., Guittonneau, S., Béguin, F., Duclaux, L., 2016. Single, binary, and mixture adsorption of nine organic contaminants onto a microporous and a microporous / mesoporous activated carbon cloth. Microporous and Mesoporous Materials 234, 24-34.

Matilainen, A., Iivari, P., Sallanko, J., Heiska, E., Tuhkanen, T., 2006. The role of ozonation and activated carbon filtration in the natural organic matter removal from drinking water. Environmental Technology 27, 1171-1180.

McBride, S.G., Osburn, E.D., Barrett, J.E., Strickland, M.S., 2019. Volatile methanol and acetone additions increase labile soil carbon and inhibit nitrification. Biogeochemistry 145, 127-140.

Meinel, F., Ruhl, A.S., Sperlich, A., Zietzschmann, F., Jekel, M., 2015. Pilot-scale investigation of micropollutant removal with granular and powdered activated carbon. Water, Air, \& Soil Pollution 226, 1-10.

Middelhoven, W.J., Doesburg, W., 2007. Utilization of hexamethylenetetramine (urotropine) by bacteria and yeasts. Antonie van Leeuwenhoek, International Journal of General and Molecular Microbiology 91, 191-196.

Miklos, D.B., Remy, C., Jekel, M., Linden, K.G., Drewes, J.E., Hübner, U., 2018. Evaluation of advanced oxidation processes for water and wastewater treatment - A critical review. Water Research 139, 118-131.

Mo, W., Cornejo, P.K., Malley, J.P., Kane, T.E., Collins, M.R., 2018. Life cycle environmental and economic implications of small drinking water system upgrades to reduce disinfection byproducts. Water Research 143, 155-164.

Mompelat, S., Le Bot, B., Thomas, O., 2009. Occurrence and fate of pharmaceutical products and by-products, from resource to drinking water. Environment International 35, 803-814.

Mondal, S., Samajdar, R.N., Mukherjee, S., Bhattacharyya, A.J., Bagchi, B., 2018. Unique features of metformin: a combined experimental, theoretical, and simulation study of its structure, dynamics, and interaction energetics with DNA grooves. The Journal of Physical Chemistry B 122, 2227-2242. 
Moreno-Castilla, C., 2004. Adsorption of organic molecules from aqueous solutions on carbon materials. Carbon 42, 83-94.

Mousel, D., Palmowski, L., Pinnekamp, J., 2017. Energy demand for elimination of organic micropollutants in municipal wastewater treatment plants. Science of the Total Environment $575,1139-1149$.

Müller, J., Jewell, K.S., Schulz, M., Hermes, N., Ternes, T.A., Drewes, J.E., Hübner, U., 2019. Capturing the oxic transformation of iopromide - A useful tool for an improved characterization of predominant redox conditions and the removal of trace organic compounds in biofiltration systems? Water Research 152, 274-284.

$\mathbf{N}$

Nakagawa, K., Namba, A., Mukai, S.R., Tamon, H., Ariyadejwanich, P., Tanthapanichakoon, W., 2004. Adsorption of phenol and reactive dye from aqueous solution on activated carbons derived from solid wastes. Water Research 38, 1791-1798.

Nam, S.-W., Choi, D.-J., Kim, S.-K., Her, N., Zoh, K.-D., 2014. Adsorption characteristics of selected hydrophilic and hydrophobic micropollutants in water using activated carbon. Journal of Hazardous Materials 270, 144-52.

Narbaitz, R.M., Cen, J., 1997. Alternative methods for determining the percentage regeneration of activated carbon. Water Research 31, 2532-2542.

Nath, K., Bhakhar, M.S., 2011. Microbial regeneration of spent activated carbon dispersed with organic contaminants: Mechanism, efficiency, and kinetic models. Environmental Science and Pollution Research 18, 534-546.

Nguyen, L.N., Hai, F.I., Dosseto, A., Richardson, C., Price, W.E., Nghiem, L.D., 2016. Continuous adsorption and biotransformation of micropollutants by granular activated carbon-bound laccase in a packed-bed enzyme reactor. Bioresource Technology 210, 108116.

Nguyen, L.N., Hai, F.I., Kang, J., Price, W.E., Nghiem, L.D., 2012. Removal of trace organic contaminants by a membrane bioreactor-granular activated carbon (MBR-GAC) system. Bioresource Technology 113, 169-173.

Nieuwenhuizen, C. van, 2017. Omgevingsweb Kamerbrief over innamestops drinkwater [WWW Document]. URL https://www.omgevingsweb.nl/nieuws/kamerbrief-overinnamestops-drinkwater (accessed 3.20.18).

Nord, N.B., Bester, K., 2020. Can the removal of pharmaceuticals in biofilters be influenced by short pulses of carbon? Science of the Total Environment 707, 135901.

\section{$\mathbf{O}$}

Ocampo-Pérez, R., Abdel daiem, M.M., Rivera-Utrilla, J., Méndez-Díaz, J.D., Sánchez-Polo, M., 2012a. Modeling adsorption rate of organic micropollutants present in landfill leachates onto granular activated carbon. Journal of Colloid and Interface Science 385, 174-182.

Ocampo-Pérez, R., Leyva-Ramos, R., Alonso-Davila, P., Rivera-Utrilla, J., Sanchez-Polo, M., 
2010. Modeling adsorption rate of pyridine onto granular activated carbon. Chemical Engineering Journal 165, 133-141.

Ocampo-Pérez, R., Leyva-Ramos, R., Mendoza-Barron, J., Guerrero-Coronado, R.M., 2011. Adsorption rate of phenol from aqueous solution onto organobentonite: Surface diffusion and kinetic models. Journal of Colloid and Interface Science 364, 195-204.

Ocampo-Pérez, R., Leyva-Ramos, R., Rivera-Utrilla, J., Flores-Cano, J. V., Sánchez-Polo, M., 2015. Modeling adsorption rate of tetracyclines on activated carbons from aqueous phase. Chemical Engineering Research and Design 104, 579-588.

Ocampo-Pérez, R., Rivera-Utrilla, J., Gómez-Pacheco, C., Sánchez-Polo, M., López-Peñalver, J.J., 2012b. Kinetic study of tetracycline adsorption on sludge-derived adsorbents in aqueous phase. Chemical Engineering Journal 213, 88-96.

Oh, W. Da, Lim, P.E., Seng, C.E., Sujari, A.N.A., 2011. Bioregeneration of granular activated carbon in simultaneous adsorption and biodegradation of chlorophenols. Bioresource Technology 102, 9497-9502.

Oh, S., Hammes, F., Liu, W., 2018. Metagenomic characterization of biofilter microbial communities in a full-scale drinking water treatment plant. Water Research 128, 278-285.

Oh, W.D., Lim, P.E., Seng, C.E., Sujari, A.N.A., 2012. Kinetic modeling of bioregeneration of chlorophenol-loaded granular activated carbon in simultaneous adsorption and biodegradation processes. Bioresource Technology 114, 179-187.

Örn, S., Holbech, H., Madsen, T.H., Norrgren, L., Petersen, G.I., 2003. Gonad development and vitellogenin production in zebrafish (Danio rerio) exposed to ethinylestradiol and methyltestosterone. Aquatic Toxicology 65, 397-411.

Östman, M., Björlenius, B., Fick, J., Tysklind, M., 2019. Effect of full-scale ozonation and pilotscale granular activated carbon on the removal of biocides, antimycotics and antibiotics in a sewage treatment plant. Science of the Total Environment 649, 1117-1123.

$\mathbf{P}$

Pawlowski, S., Van Aerle, R., Tyler, C.R., Braunbeck, T., 2004. Effects of $17 \alpha$-ethinylestradiol in a fathead minnow (Pimephales promelas) gonadal recrudescence assay. Ecotoxicology and Environmental Safety 57, 330-345.

Pelekani, C., Snoeyink, V.L., 1999. Competitive adsorption in natural water: Role of activated carbon pore size. Water Research 33, 1209-1219.

Pérez, S., Barceló, D., 2007. Fate and occurrence of X-ray contrast media in the environment. Analytical and Bioanalytical Chemistry 387, 1235-1246.

Piai, L., Blokland, M., van der Wal, A., Langenhoff, A., 2020a. Biodegradation and adsorption of micropollutants by biological activated carbon from a drinking water production plant. Journal of Hazardous Materials 388, 122028.

Piai, L., Dykstra, J.E., Adishakti, M.G., Blokland, M., Langenhoff, A.A.M., van der Wal, A., 2019. Diffusion of hydrophilic organic micropollutants in granular activated carbon with 
different pore sizes. Water Research 162, 518-527.

Piai, L., van der Wal, A., Boelee, N., Langenhoff, A., 2020b. Melamine degradation to bioregenerate granular activated carbon. Submitted to Journal of Hazardous Materials.

Pikaar, I., Koelmans, A.A., Noort, P.C.M. Van, 2006. Sorption of organic compounds to activated carbons. Evaluation of isotherm models. Chemosphere 65, 2343-2351.

Poursat, B.A.J., van Spanning, R.J.M., Braster, M., Helmus, R., de Voogt, P., Parsons, J.R., 2019. Biodegradation of metformin and its transformation product, guanylurea, by natural and exposed microbial communities. Ecotoxicology and Environmental Safety 182, 109414.

Putz, A.R.H., Losh, D.E., Speitel, G.E., 2005. Removal of nonbiodegradable chemicals from mixtures during granular activated carbon bioregeneration. Journal of Environmental Engineering 131, 196-205.

$\mathbf{R}$

Radovic, L.R., Silva, I.F., Ume, J.I., Menéndez, J.A., Leon Y Leon, C.A., Scaroni, A.W., 1997. An experimental and theoretical study of the adsorption of aromatics possessing electronwithdrawing and electron-donating functional groups by chemically modified activated carbons. Carbon 35, 1339-1348.

Rattier, M., Reungoat, J., Keller, J., Gernjak, W., 2014. Removal of micropollutants during tertiary wastewater treatment by biofiltration: Role of nitrifiers and removal mechanisms. Water Research 54, 89-99.

Redding, A.M., Cannon, F.S., Snyder, S.A., Vanderford, B.J., 2009. A QSAR-like analysis of the adsorption of endocrine disrupting compounds, pharmaceuticals, and personal care products on modified activated carbons. Water Research 43, 3849-3861.

Redeker, M., Wick, A., Meermann, B., Ternes, T.A., 2018. Anaerobic transformation of the iodinated X-ray contrast medium iopromide, its aerobic transformation products, and transfer to further iodinated X-ray contrast media. Environmental Science E Technology 52, 8309-8320.

Reemtsma, T., Berger, U., Arp, H.P.H., Gallard, H., Knepper, T.P., Neumann, M., Quintana, J.B., Voogt, P. De, 2016. Mind the gap: persistent and mobile organic compounds - water contaminants that slip through. Environmental Science \& Technology 50, 10308-10315.

Ren, X., Kou, Y.Y., Kim, T., Chae, K.J., Ng, H.Y., 2017. Toxicity study of reclaimed water on human embryonic kidney cells. Chemosphere 189, 390-398.

Reungoat, J., Escher, B.I., Macova, M., Argaud, F.X., Gernjak, W., Keller, J., 2012. Ozonation and biological activated carbon filtration of wastewater treatment plant effluents. Water Research 46, 863-872.

Reungoat, J., Escher, B.I., Macova, M., Keller, J., 2011. Biofiltration of wastewater treatment plant effluent: Effective removal of pharmaceuticals and personal care products and reduction of toxicity. Water Research 45, 2751-2762.

Reungoat, J., Macova, M., Escher, B.I., Carswell, S., Mueller, J.F., Keller, J., 2010. Removal of 
micropollutants and reduction of biological activity in a full scale reclamation plant using ozonation and activated carbon filtration. Water Research 44, 625-637.

RIWA-Maas, 2017. Jaarrapport 2017 De Maas.

RIWA-Maas, 2016. Jaarrapport 2016 - De Maas.

RIWA-Rijn, 2018. Jaarrapport 2018 - De Rijn.

RIWA-Rijn, 2016. Jaarrapport 2016 De Rijn.

Rossner, A., Snyder, S.A., Knappe, D.R.U., 2009. Removal of emerging contaminants of concern by alternative adsorbents. Water Research 43, 3787-3796.

Roy, D., Maillacheruvu, K., Mouthon, J., 1999. Bioregeneration of granular activated carbon loaded with 2,4-D. Journal of Environmental Science \& Health - Part B 34, 769-791.

Ruff, M., Mueller, M.S., Loos, M., Singer, H.P., 2015. Quantitative target and systematic nontarget analysis of polar organic micro-pollutants along the river Rhine using highresolution mass- spectrometry - Identification of unknown sources and compounds. Water Research 87, 145-154.

\section{$S$}

Sander, M., Pignatello, J.J., 2005. Characterization of charcoal adsorption sites for aromatic compounds: Insights drawn from single-solute and bi-solute competitive experiments. Environmental Science \& Technology 39, 1606-1615.

Santos, L.H.M.L.M., Araújo, A.N., Fachini, A., Pena, A., Delerue-Matos, C., Montenegro, M.C.B.S.M., 2010. Ecotoxicological aspects related to the presence of pharmaceuticals in the aquatic environment. Journal of Hazardous Materials 175, 45-95.

Scharf, R.G., Johnston, R.W., Semmens, M.J., Hozalski, R.M., 2010. Comparison of batch sorption tests, pilot studies, and modeling for estimating GAC bed life. Water Research 44, 769-780.

Scherrenberg, S.M., Neef, R., Menkveld, H.W.H., van Nieuwenhuijzen, A.F., van der Graaf, J.H.J.M., 2012. Investigating phosphorus limitation in a fixed bed filter with phosphorus and nitrogen profile measurements. Water Environment Research 84, 25-33.

Scheurer, M., Michel, A., Brauch, H.-J., Ruck, W., Sacher, F., 2012. Occurrence and fate of the antidiabetic drug metformin and its metabolite guanylurea in the environment and during drinking water treatment. Water Research 46, 4790-4802.

Scheurer, M., Sacher, F., Brauch, H.-J., 2009. Occurrence of the antidiabetic drug metformin in sewage and surface waters in Germany. Journal of Environmental Monitoring 11, 1608-1613.

Scholz, M., Martin, R.J., 1997. Ecological equilibrium on biological activated carbon. Water Research 31, 2959-2968.

Schulz, M., Loffler, D., Wagner, M., Ternes, T.A., 2008. Transformation of the X-ray contrast medium iopromide in soil and biological wastewater treatment. Environmental Science $\mathcal{E}$ Technology 42, 7207-7217. 
Schulze, S., Zahn, D., Montes, R., Rodil, R., Quintana, J.B., Knepper, T.P., Reemtsma, T., Berger, U., 2019. Occurrence of emerging persistent and mobile organic contaminants in European water samples. Water Research 153, 80-90.

Seitz, W., Winzenbacher, R., 2017. A survey on trace organic chemicals in a German water protection area and the proposal of relevant indicators for anthropogenic influences. Environmental Monitoring and Assessment 189, 244.

Shelton, D.R., Karns, J.S., Mccarty, G.W., Durham, D.R., 1997. Metabolism of melamine by Klebsiella terragena. Applied and Environmental Microbiology 63, 2832-2835.

Shen, L., Lu, Y., Liu, Y., 2012. Mathematical modeling of biofilm-covered granular activated carbon: A review. Journal of Chemical Technology and Biotechnology 87, 1513-1520.

Shi, Z.Q., Liu, Y.S., Xiong, Q., Cai, W.W., Ying, G.G., 2019. Occurrence, toxicity and transformation of six typical benzotriazoles in the environment: A review. Science of the Total Environment 661, 407-421.

Shimabuku, K.K., Kennedy, A.M., Mulhern, R.E., Summers, R.S., 2017a. Evaluating activated carbon adsorption of dissolved organic matter and micropollutants using fluorescence spectroscopy. Environmental Science E Technology 51, 2676-2684.

Shimabuku, K.K., Paige, J.M., Luna Agüero, M., Summers, R.S., 2017b. Simplified modeling of organic contaminant adsorption by activated carbon and biochar in the presence of dissolved organic matter and other competing adsorbates. Environmental Science $\mathcal{E}$ Technology 51, 10031-10040.

Shiomi, N., Ako, M., 2012. Biodegradation of melamine and cyanuric acid by a newly-isolated microbacterium strain. Advances in Microbiology 02, 303-309.

Sirotkin, A.S., Koshkina, L.Y., Ippolitov, K.G., 2001. The BAC-process for treatment of waste water containing non-ionogenic synthetic surfactants. Water Research 35, 3265-3271.

Sjerps, R.M.A., ter Laak, T.L., Zwolsman, G.J.J.G., 2017. Projected impact of climate change and chemical emissions on the water quality of the European rivers Rhine and Meuse: A drinking water perspective. Science of the Total Environment 601-602, 1682-1694.

Sjerps, R.M.A., Vughs, D., van Leerdam, J.A., ter Laak, T.L., van Wezel, A.P., 2016. Data-driven prioritization of chemicals for various water types using suspect screening LC-HRMS. Water Research 93, 254-264.

Smit, C.E. (RIVM), 2018. Water quality standards for melamine A proposal in accordance with the methodology of the Water Framework Directive. Bilthoven.

Smolin, S., Kozyatnyk, I., Klymenko, N., 2020. New approach for the assessment of the contribution of adsorption, biodegradation and self-bioregeneration in the dynamic process of biologically active carbon functioning. Chemosphere 248, 126022.

Soliman, M., Eldyasti, A., 2018. Ammonia-Oxidizing Bacteria (AOB): opportunities and applications - a review. Reviews in Environmental Science and Biotechnology 17, 285-321.

Sompornpailin, D., Ratanatawanate, C., Sattayanon, C., Namuangruk, S., Punyapalakul, P., 
2020. Selective adsorption mechanisms of pharmaceuticals on benzene-1,4-dicarboxylic acid-based MOFs: Effects of a flexible framework, adsorptive interactions and the DFT study. Science of the Total Environment 720, 137449.

Souza, P.R., Dotto, G.L., Salau, N.P.G., 2017. Detailed numerical solution of pore volume and surface diffusion model in adsorption systems. Chemical Engineering Research and Design 122, 298-307.

Speitel, G.E., Dovantzis, K., DiGiano, F.A., 1987. Mathematical modeling of bioregeneration in GAC columns. Journal of Environmental Engineering (United States) 113, 32-48.

Sperlich, A., Harder, M., Zietzschmann, F., Gnirss, R., 2017. Fate of trace organic compounds in granular activated carbon (GAC) adsorbers for drinking water treatment. Water 9, 479.

Stackelberg, P.E., Furlong, E.T., Meyer, M.T., Zaugg, S.D., Henderson, A.K., Reissman, D.B., 2004. Persistence of pharmaceutical compounds and other organic wastewater contaminants in a conventional drinking-water-treatment plant. Science of the Total Environment 329, 99-113.

Stackelberg, P.E., Gibs, J., Furlong, E.T., Meyer, M.T., Zaugg, S.D., Lippincott, R.L., 2007. Efficiency of conventional drinking-water-treatment processes in removal of pharmaceuticals and other organic compounds. Science of the Total Environment 377, 255272.

Starling, M.C.V.M., Amorim, C.C., Leão, M.M.D., 2019. Occurrence, control and fate of contaminants of emerging concern in environmental compartments in Brazil. Journal of Hazardous Materials 372, 17-36.

Stuart, M., Lapworth, D., Crane, E., Hart, A., 2012. Review of risk from potential emerging contaminants in UK groundwater. Science of the Total Environment 416, 1-21.

Suarez, S., Lema, J.M., Omil, F., 2010. Removal of pharmaceutical and personal care products (PPCPs) under nitrifying and denitrifying conditions. Water Research 44, 3214-3224.

Sun, S., Jiang, T., Lin, Y., Song, J., Zheng, Y., An, D., 2020. Characteristics of organic pollutants in source water and purification evaluations in drinking water treatment plants. Science of the Total Environment 733, 139277.

Suzuki, I., Kwok, S.-C., Dular, U., 1976. Competitive inhibition of ammonia oxidation in Nitrosomonas europaea by methane, carbon monoxide or methanol. FEBS Letters 72, 117120.

\section{$\mathbf{T}$}

Tadkaew, N., Hai, F.I., McDonald, J.A., Khan, S.J., Nghiem, L.D., 2011. Removal of trace organics by MBR treatment: The role of molecular properties. Water Research 45, 2439 2451.

Taghdiri, N., Zamani, M., 2013. Hexamine adsorption study on activated carbon from aqueous solutions for application in treatment of hexamine industrial wastewater. International Journal of Environmental Science and Technology 10, 19-26. 
Takagi, K., Fujii, K., Yamazaki, K.I., Harada, N., Iwasaki, A., 2012. Biodegradation of melamine and its hydroxy derivatives by a bacterial consortium containing a novel Nocardioides species. Applied Microbiology and Biotechnology 94, 1647-1656.

Tang, K., Escola Casas, M., Ooi, G.T.H., Kaarsholm, K.M.S., Bester, K., Andersen, H.R., 2017. Influence of humic acid addition on the degradation of pharmaceuticals by biofilms in effluent wastewater. International Journal of Hygiene and Environmental Health 220, 604-610.

ter Laak, T.L., Kooij, P.J.F., Tolkamp, H., Hofman, J., 2014. Different compositions of pharmaceuticals in Dutch and Belgian rivers explained by consumption patterns and treatment efficiency. Environmental Science and Pollution Research 21, 12843-12855.

Ternes, T., 2007. The occurrence of micopollutants in the aquatic environment: a new challenge for water management. Water Science \& Technology 55, 327-332.

Ternes, T.A., Meisenheimer, M., McDowell, D., Sacher, F., Brauch, H.-J., Haist-Gulde, B., Preuss, G., Wilme, U., Zulei-Seibert, N., 2002. Removal of pharmaceuticals during drinking water treatment. Environmental Science \& Technology 36, 3855-3863.

Thomson, J., Roddick, F.A., Drikas, M., 2002. UV photooxidation facilitating biological treatment for the removal of NOM from drinking water. Journal of Water Supply: Research and Technology - AQUA 51, 297-306.

Tisler, S., Zwiener, C., 2019. Aerobic and anaerobic formation and biodegradation of guanylurea and other transformation products of metformin. Water Research 149, 130-135.

To, P.C., Mariñas, B.J., Snoeyink, V.L., Wun, J.N., 2008a. Effect of pore-blocking background compounds on the kinetics of trace organic contaminant desorption from activated carbon. Environmental Science E Technology 42, 4825-4830.

To, P.C., Mariñas, B.J., Snoeyink, V.L., Wun, J.N., 2008b. Effect of strongly competing background compounds on the kinetics of trace organic contaminant desorption from activated carbon. Environmental Science \& Technology 42, 4825-4830.

Toh, R.H., Lim, P.E., Seng, C.E., Adnan, R., 2013. Immobilized acclimated biomass-powdered activated carbon for the bioregeneration of granular activated carbon loaded with phenol and o-cresol. Bioresource Technology 143, 265-274.

Torresi, E., Polesel, F., Bester, K., Christensson, M., Smets, B.F., Trapp, S., Andersen, H.R., Plósz, B.G., 2017. Diffusion and sorption of organic micropollutants in biofilms with varying thicknesses. Water Research 123, 388-400.

Tran, H.N., You, S., Hosseini-bandegharaei, A., 2017. Mistakes and inconsistencies regarding adsorption of contaminants from aqueous solutions : A critical review. Water Research 120, 88-116.

Tran, N.H., Urase, T., Ngo, H.H., Hu, J., Ong, S.L., 2013. Insight into metabolic and cometabolic activities of autotrophic and heterotrophic microorganisms in the biodegradation of emerging trace organic contaminants. Bioresource Technology 146, 721-731.

Trautwein, C., Kümmerer, K., 2011. Incomplete aerobic degradation of the antidiabetic drug Metformin and identification of the bacterial dead-end transformation product 
Guanylurea. Chemosphere 85, 765-773.

Trenholm, R.A., Vanderford, B.J., Holady, J.C., Rexing, D.J., Snyder, S.A., 2006. Broad range analysis of endocrine disruptors and pharmaceuticals using gas chromatography and liquid chromatography tandem mass spectrometry. Chemosphere 65, 1990-1998.

Triebskorn, R., Casper, H., Scheil, V., Schwaiger, J., 2007. Ultrastructural effects of pharmaceuticals (carbamazepine, clofibric acid, metoprolol, diclofenac) in rainbow trout (Oncorhynchus mykiss) and common carp (Cyprinus carpio). Analytical and Bioanalytical Chemistry 387, 1405-1416. I

Tröger, R., Klöckner, P., Ahrens, L., Wiberg, K., 2018. Micropollutants in drinking water from source to tap - Method development and application of a multiresidue screening method. Science of the Total Environment 627, 1404-1432.

Tröger, R., Köhler, S.J., Franke, V., Bergstedt, O., Wiberg, K., 2020. A case study of organic micropollutants in a major Swedish water source - Removal efficiency in seven drinking water treatment plants and influence of operational age of granulated active carbon filters. Science of the Total Environment 706, 135680.

V

Vakili, M., Mojiri, A., Kindaichi, T., Cagnetta, G., Yuan, J., Wang, B., Giwa, A.S., 2019. Crosslinked chitosan/zeolite as a fixed-bed column for organic micropollutants removal from aqueous solution, optimization with RSM and artificial neural network. Journal of Environmental Management 250, 109434.

Valderrama, C., Gamisans, X., de las Heras, X., Farrán, A., Cortina, J.L., 2008. Sorption kinetics of polycyclic aromatic hydrocarbons removal using granular activated carbon: Intraparticle diffusion coefficients. Journal of Hazardous Materials 157, 386-396.

Velten, S., Boller, M., Ko, O., Helbing, J., Weilenmann, H., Hammes, F., 2011a. Development of biomass in a drinking water granular active carbon (GAC) filter. Water Research 5, 4-11.

Velten, S., Knappe, D.R.U., Traber, J., Kaiser, H.P., von Gunten, U., Boller, M., Meylan, S., 2011b. Characterization of natural organic matter adsorption in granular activated carbon adsorbers. Water Research 45, 3951-3959.

Villacañas, F., Pereira, M.F.R., Órfão, J.J.M., Figueiredo, J.L., 2006. Adsorption of simple aromatic compounds on activated carbons. Journal of Colloid and Interface Science 293, 128136.

Vinitnantharat, S., Baral, A., Ishibashi, Y., Ha, S.R., 2001. Quantitative bioregeneration of granular activated carbon loaded with phenol and 2,4-dichlorophenol. Environmental Technology 22, 339-344.

Völker, J., Castronovo, S., Wick, A., Ternes, T.A., Joss, A., Oehlmann, J., Wagner, M., 2016. Advancing biological wastewater treatment: extended anaerobic conditions enhance the removal of endocrine and dioxin-like activities. Environmental Science E Technology 50, 10606-10615.

Völker, J., Vogt, T., Castronovo, S., Wick, A., Ternes, T.A., Joss, A., Oehlmann, J., Wagner, M., 
2017. Extended anaerobic conditions in the biological wastewater treatment: Higher reduction of toxicity compared to target organic micropollutants. Water Research 116, 220 230.

Voysey, P.A., Wood, P.M., 1987. Methanol and formaldehyde oxidation by an autotrophic nitrifying bacterium. Journal of General Microbiology 44, 283-290.

\section{W}

Wagner, T. V, Parsons, J.R., Rijnaarts, H.H.M., Voogt, P. De, Langenhoff, A.A.M., 2020. Benzotriazole removal mechanisms in pilot-scale constructed wetlands treating cooling tower water. Journal of Hazardous Materials 384, 121314.

Wang, H., Ho, L., Lewis, D.M., Brookes, J.D., Newcombe, G., 2007. Discriminating and assessing adsorption and biodegradation removal mechanisms during granular activated carbon filtration of microcystin toxins. Water Research 41, 4262-4270.

Wang, H., Li, J., Hu, A., Qin, D., Xu, H., Yu, C.P., 2014. Melaminivora alkalimesophila gen. nov., sp. nov., a melamine-degrading betaproteobacterium isolated from a melamine-producing factory. International Journal of Systematic and Evolutionary Microbiology 64, 1938-1944.

Wang, L., Li, J., 2013. Adsorption of C.I. Reactive Red 228 dye from aqueous solution by modified cellulose from flax shive: Kinetics, equilibrium, and thermodynamics. Industrial Crops and Products 42, 153-158.

Wigton, A., Kilduff, J.E., 2004. Modeling trichloroethylene adsorption by activated carbon preloaded with natural dissolved organic matter using a modified IAST approach. Environmental Science \& Technology 38, 5825-5833.

Wilke, C.R., Chang, P., 1955. Correlation of diffusion coefficients in dilute solutions. A.I.Ch.E. Journal 1, 264-270.

Winkler, M.K., Straka, L., 2019. New directions in biological nitrogen removal and recovery from wastewater. Current Opinion in Biotechnology 57, 50-55.

Wohler, L., Niebaum, G., Krol, M., Hoekstra, A.Y., Lara, W., 2020. The grey water footprint of human and veterinary pharmaceuticals. Water Research 7, 100044.

Worch, E., 2012. Adsorption technology in water treatment. Fundamentals, processes and modeling. Walter de Gruyter GmbH \& Co. KG, Berlin/Boston.

Wunder, D.B., Bosscher, V.A., Cok, R.C., Hozalski, R.M., 2011. Sorption of antibiotics to biofilm. Water Research 45, 2270-2280.

\section{$\mathbf{X}$}

Xiaojian, Z., Zhansheng, W., Xiasheng, G., 1991. Simple combination of biodegradation and carbon adsorption-the mechanism of the biological activated carbon process. Water Research 25, 165-172.

Xu, S., Zhang, Y., Sims, A., Bernards, M., Hu, Z., 2013. Fate and toxicity of melamine in activated sludge treatment systems after a long-term sludge adaptation. Water Research 47, 2307-2314. 
Xu, S., Zhou, S., Xing, L., Shi, P., Shi, W., Zhou, Q., Pan, Y., 2019. Fate of organic micropollutants and their biological effects in a drinking water source treated by a field-scale constructed wetland. Science of the Total Environment 682, 756-764.

$\mathbf{Y}$

Ye, N., Cimetiere, N., Heim, V., Fauchon, N., Feliers, C., Wolbert, D., 2019. Upscaling fixed bed adsorption behaviors towards emerging micropollutants in treated natural waters with aging activated carbon: Model development and validation. Water Research 148, 30-40.

Yin, C.Y., Aroua, M.K., Daud, W.M.A.W., 2007. Review of modifications of activated carbon for enhancing contaminant uptakes from aqueous solutions. Separation and Purification Technology 52, 403-415.

Yu, Y., Han, P., Zhou, L.J., Li, Z., Wagner, M., Men, Y., 2018. Ammonia monooxygenasemediated cometabolic biotransformation and hydroxylamine-mediated abiotic transformation of micropollutants in an AOB/NOB co-culture. Environmental Science $\mathcal{E}$ Technology 52, 9196-9205.

Yuan, X., Zhuo, S.-P., Xing, W., Cui, H.-Y., Dai, X.-D., Liu, X.-M., Yan, Z.-F., 2007. Aqueous dye adsorption on ordered mesoporous carbons. Journal of Colloid and Interface Science 310, 8389.

\section{Z}

Zhang, L., Carvalho, P.N., Bollmann, U.E., EI-taliawy, H., Brix, H., Bester, K., 2019. Enhanced removal of pharmaceuticals in a biofilter: Effects of manipulating co-degradation by carbon feeding. Chemosphere 236, 124303.

Zhang, L., Dong, D., Hua, X., Guo, Z., 2018. Inhibitory effects of extracellular polymeric substances on ofloxacin sorption by natural biofilms. Science of the Total Environment 625, 178-184.

Zhang, Q., Crittenden, J., Hristovski, K., Hand, D., Westerhoff, P., 2009. User-oriented batch reactor solutions to the homogeneous surface diffusion model for different activated carbon dosages. Water Research 43, 1859-1866.

Zhang, S., Gitungo, S.W., Axe, L., Raczko, R.F., Dyksen, J.E., 2017. Biologically active filters - An advanced water treatment process for contaminants of emerging concern. Water Research $114,31-41$.

Zhiteneva, V., Ziemendorf, É., Sperlich, A., Drewes, J.E., Hübner, U., 2020. Differentiating between adsorption and biodegradation mechanisms while removing trace organic chemicals (TOrCs) in biological activated carbon (BAC) filters. Science of the Total Environment 743, 140567.

Zhu, H., Lee, S., Moon, H., Kannan, K., 2019. Spatial and temporal trends of melamine and its derivatives in sediment from Lake Shihwa, South Korea. Journal of Hazardous Materials $373,671-677$.

Zhu, Q., Moggridge, G.D., Ainte, M., Mantle, M.D., Gladden, L.F., D’Agostino, C., 2016. Adsorption of pyridine from aqueous solutions by polymeric adsorbents MN 200 and 
MN 500. Part 2: Kinetics and diffusion analysis. Chemical Engineering Journal 306, 67-76.

Zietzschmann, F., Aschermann, G., Jekel, M., 2016a. Comparing and modeling organic micropollutant adsorption onto powdered activated carbon in different drinking waters and WWTP effluents. Water Research 102, 190-201.

Zietzschmann, F., Mitchell, R.-L., Jekel, M., 2015. Impacts of ozonation on the competition between organic micro-pollutants and effluent organic matter in powdered activated carbon adsorption. Water Research 84, 153-160.

Zietzschmann, F., Stützer, C., Jekel, M., 2016b. Granular activated carbon adsorption of organic micro-pollutants in drinking water and treated wastewater - Aligning breakthrough curves and capacities. Water Research 92, 180-187.

Zietzschmann, F., Worch, E., Altmann, J., Ruhl, A.S., Sperlich, A., Meinel, F., Jekel, M., 2014. Impact of EfOM size on competition in activated carbon adsorption of organic micropollutants from treated wastewater. Water Research 65, 297-306. 


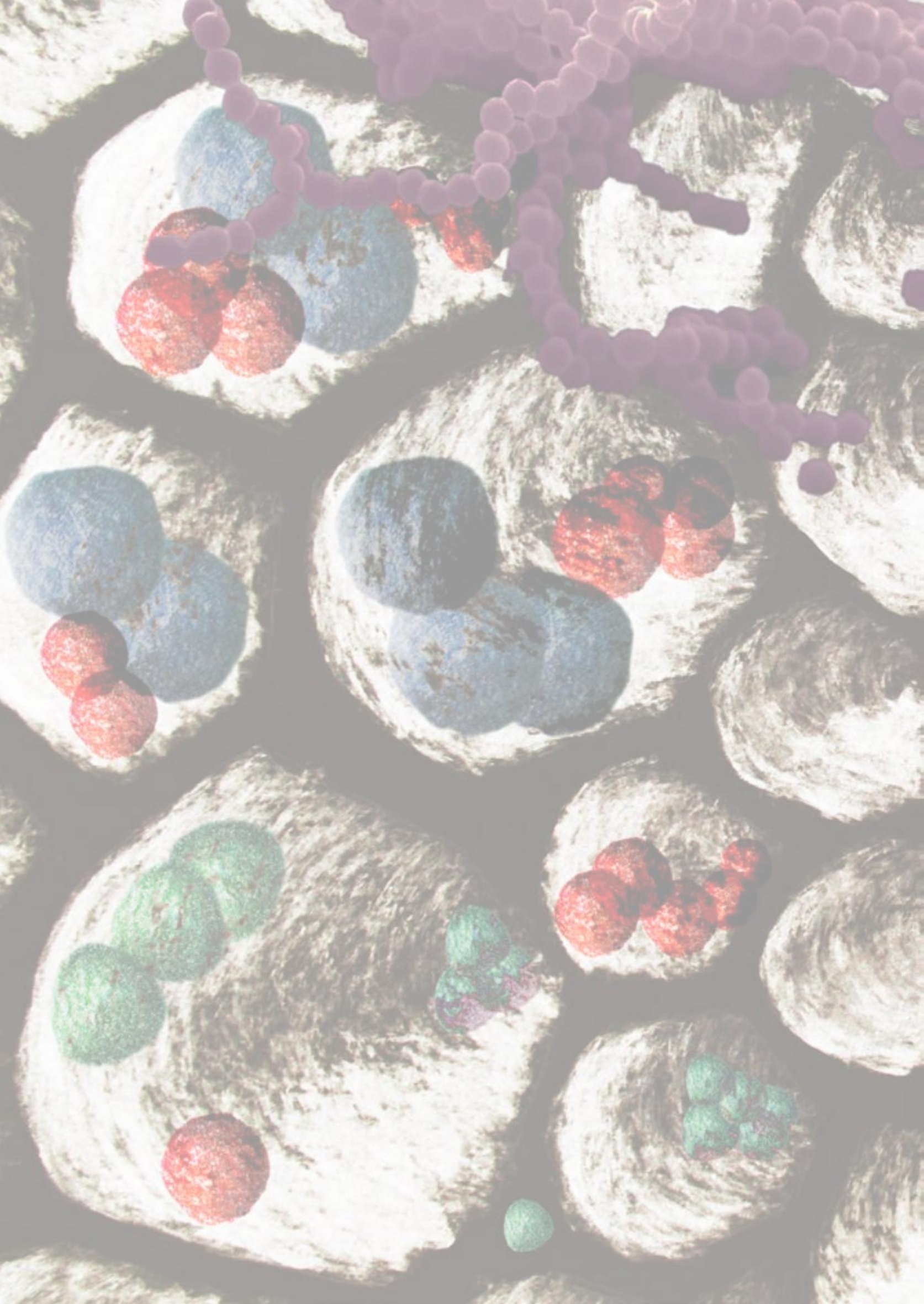




\section{Summary}

The presence of micropollutants in surface water threatens the production of high quality and safe drinking water. Micropollutants are contaminants present in the environment at trace concentrations $(\mathrm{ng} / \mathrm{L}-\mu \mathrm{g} / \mathrm{L})$. These contaminants originate from the use of organic chemicals in anthropogenic activities. Micropollutants reach the environment due to incomplete removal in wastewater treatment plants or via diffuse sources, such as agricultural and urban water runoff and sewer overflow. Despite their occurrence at trace concentrations, they can negatively impact the environment. Moreover, they can impact human health when water contaminated with micropollutants is used to produce drinking water. To minimize these risks, it is crucial to prevent micropollutants from reaching the environment and our drinking water.

Micropollutants can be removed from water using adsorption to granular activated carbon (GAC) in fixed-bed filters. GAC filters are often applied as a polishing step in the production of drinking water from surface water. GAC adsorption capacity reduces with time, hence GAC needs to be reactivated regularly to maintain high removal efficiencies. GAC reactivation is achieved by oxidizing the adsorbed contaminants at high temperatures $\left(>700^{\circ} \mathrm{C}\right)$, which makes it an energy intensive process, resulting in large carbon footprints. Hence, efforts to increase sustainability in GAC filtration should focus on reducing the need for GAC reactivation.

During the use of GAC filters, biomass inevitably grows on the GAC surface and contributes to removing the biodegradable fraction of organic contaminants, including biodegradable micropollutants. Biodegradation and adsorption are synergistic processes. Biodegradation of micropollutants from the liquid phase reduces the amount of molecules that adsorb to the GAC. Furthermore, biodegradation of previously adsorbed micropollutants releases adsorption sites, regenerating GAC adsorption capacity and reducing the need for reactivation. This thesis addresses the potential of combining adsorption and biodegradation of micropollutants to bioregenerate GAC.

In Chapter 2, the adsorption capacity and rate of adsorption of hydrophilic micropollutants to fresh GAC is assessed. Micropollutants removal in GAC filters is not only determined by equilibrium characteristics, such as affinity for the GAC and the adsorption capacity, but also by the adsorption rate. The rate-determining step in adsorption using GAC is usually 
the adsorbate diffusion inside the granules. The presence of mesopores can facilitate diffusion, resulting in higher adsorption rates. In this thesis, we use two different types of GAC, with and without mesopores, to study the adsorption rate of hydrophilic micropollutants. Ten micropollutants are selected as model compounds: benzotriazole, desphenyl-chloridazon, diclofenac, guanylurea, hexamethylenetetramine, iopamidol, iopromide, melamine, metformin and pyrazole. A pore diffusion model is applied to the kinetic data to obtain pore diffusion coefficients. This chapter shows that adsorption rate is influenced by the molecular size of the micropollutant as well as the GAC pore size. Pore diffusion coefficients correlate negatively with the adsorbate size for most micropollutants and this correlation is stronger for the largest adsorbates. Furthermore, diffusion of the largest micropollutants is hindered in the GAC micropores. Micropore surface area increases GAC adsorption capacity for micropollutants, although size-exclusion effects were observed for the largest molecules, i.e., iopamidol and iopromide. Finally, affinity for GAC correlates to molecular structure: micropollutants with cyclic structures, e.g., benzotriazole and melamine, adsorb to GAC to a larger extent than micropollutants with linear or globular structures, e.g. metformin and hexamethylenetetramine.

Chapter 3 describes the potential of biomass obtained from full-scale GAC filters to biodegrade micropollutants. Assessing micropollutants biodegradation in the presence of GAC requires distinguishing adsorption from biodegradation. This is done by assessing micropollutants removal at $5^{\circ} \mathrm{C}$ and $20^{\circ} \mathrm{C}$ with biologically active and autoclaved GAC. At $5^{\circ} \mathrm{C}$ micropollutants are removed mainly through adsorption whereas at $20^{\circ} \mathrm{C}$ they can also be biodegraded. Autoclaved GAC is used to determine the effect of temperature on micropollutants adsorption. Here, the same micropollutants mixture as applied in $\underline{\text { Chapter } 2}$ is used. Three of these micropollutants, iopromide, iopamidol and metformin, are biodegraded by the GAC biofilm. Additionally, temperature increases adsorption of some micropollutants, e.g. iopromide and iopamidol, whereas it decreases adsorption of others, e.g. metformin and guanylurea. Finally, the adsorption capacity of fresh and used GAC are compared with each other. GAC use in a treatment plant results in decreased adsorption capacity for most micropollutants, except for the positively charged ones (metformin and guanylurea) and hexamethylenetetramine.

In Chapters 4 and 5, the potential of biodegradation to regenerate GAC adsorption capacity is assessed. This is studied in batch and in column experiments using a model micropollutant, melamine, and biomass capable of degrading it. Melamine is frequently 
detected in surface water due to its wide application and insufficient removal in conventional wastewater treatment plants. In Chapter 4 favourable conditions for melamine biodegradation are presented. Melamine biodegradation is assessed in fully oxic and anoxic, as well as in alternating oxic and anoxic conditions. Moreover, the effect of an additional carbon source on the biodegradation is determined. The most favourable conditions for melamine biodegradation are applied to bioregenerate GAC, loaded with melamine. Melamine can be biodegraded in either oxic or anoxic conditions and melamine degrading biomass can restore at least $28 \%$ of the original GAC adsorption capacity in batch systems. Furthermore, high adsorption rates obtained for bioregenerated GAC indicate that bioregeneration occurs mainly in the largest pore fraction of GAC.

The contribution of melamine biodegradation to the total removal in lab-scale GAC filters is assessed in $\underline{\text { Chapter } 5}$. The filters are inoculated with the same melamine degrading biomass used in Chapter 4. The effect of an additional carbon source (methanol) and contact time on melamine removal efficiency using two different inoculation methods is studied. Inoculation of GAC filters with melamine degrading biomass increases melamine removal efficiency by at least $29 \%$ in the absence of an additional carbon source. When the additional carbon source methanol is supplied to inoculated filters, melamine removal is almost complete (up to 99\%) and no breakthrough is observed. Furthermore, the supply of methanol to an inoculated filter that is close to saturation can stimulate bioregeneration up to $98 \%$.

The conclusions and implications related to the experimental chapters are discussed in Chapter 6, and put in perspective of the current knowledge on micropollutants removal with GAC. Finally, recommendations for future research and opportunities for using bioregeneration to improve water treatment are presented. 


\section{Resumo}

A presença de micropoluentes orgânicos em águas superficiais ameaça a produção de água segura e de qualidade para consumo humano. Micropoluentes são contaminantes originários do uso de químicos orgânicos em atividades antropogênicas e estão presentes no meio ambiente em concentrações traço (ng/L - $\mu \mathrm{g} / \mathrm{L}$ ). Esses contaminantes chegam ao meio ambiente devido à sua remoção incompleta em estações de tratamento de efluentes ou por meio de fontes difusas, como as águas de drenagem urbana e rural e transbordamentos de esgoto. Apesar de ocorrerem em concentrações traço, micropoluentes afetam negativamente o meio ambiente. Além disso, podem ter impacto na saúde humana, caso água contaminada com tais poluentes seja utilizada para produzir água para consumo humano. Para minimizar esses riscos, é necessário prevenir que micropoluentes sejam lançados ao meio ambiente e estejam presentes na água tratada.

Micropoluentes podem ser removidos da água através de adsorção em carvão ativado granular em filtros de leito fixo. Filtros de carvão ativado são comumente utilizados nas etapas finais de produção de água para consumo humano a partir de águas superficiais. A capacidade de adsorção do carvão ativado reduz com o tempo de uso e, para que seja mantida uma alta eficiência na remoção de contaminantes, é necessário que ele seja reativado regularmente. Esse processo consiste na oxidação dos contaminantes adsorvidos e ocorre em altas temperaturas $\left(>700^{\circ} \mathrm{C}\right)$, de modo que este se torna um processo de grande demanda energética, resultando em elevada pegada de carbono. Sendo assim, esforços para tornar mais sustentável a filtragem de água com carvão ativado devem focar na redução da frequência de sua reativação.

Durante o uso dos filtros de carvão ativado, ocorre a formação de biomassa em sua superfície, o que contribui para a remoção da parte biodegradável dos contaminantes orgânicos, inclusive de micropoluentes. Biodegradação e adsorção são processos sinergísticos. A biodegradação de micropoluentes da fase líquida reduz a quantidade de moléculas que adsorvem no carvão ativado. Além disso, a biodegradação de micropoluentes previamente adsorvidos libera sítios de adsorção que haviam sido ocupados, regenerando a capacidade de adsorção e reduzindo a necessidade de reativação do carvão. Esta tese aborda o potencial de se combinar adsorção e biodegradação para regenerar carvão ativado granular. 
No Capítulo 2, é avaliada a capacidade e a taxa de adsorção de micropoluentes hidrofílicos em carvão ativado virgem. O potencial de remoção de micropoluentes em filtros de carvão ativado não é determinado somente por propriedades de equilíbrio, como afinidade pelo carvão ativado e sua capacidade de adsorção, mas também pela taxa de adsorção. A etapa que determina a taxa de adsorção em carvão ativado granular é, em geral, a difusão do adsorvato dentro dos grânulos. A presença de mesoporos pode facilitar a difusão, resultando em taxas de adsorção mais altas. Nesta tese, para estudar a taxa de adsorção de micropoluentes hidrofílicos, foram utilizados dois tipos de carvão ativado granular - com e sem mesoporos. Dez micropoluentes foram selecionados como compostos modelo: benzotriazol, desfenil cloridazona, diclofenaco, guanilureia, hexametilenotetramina, iopamidol, iopromida, melamina, metformina e pirazol. Um modelo de difusão nos poros foi aplicado aos dados cinéticos de adsorção para obtenção dos coeficientes de difusão nos poros. Este capítulo demonstra que a taxa de adsorção é influenciada pelo tamanho molecular dos micropoluentes e pela distribuição de tamanho dos poros do carvão ativado. A magnitude dos coeficientes de difusão nos poros é inversamente proporcional ao tamanho do adsorvato para a maioria dos micropoluentes. Essa correlação é mais pronunciada para os adsorvatos de maiores dimensões (> $700 \mathrm{~g} / \mathrm{mol}$ ), que têm sua difusão dificultada nos microporos. A área superficial dos microporos aumenta a capacidade de adsorção de micropoluentes pelo carvão ativado, porém efeitos de exclusão por tamanho são observados para as moléculas grandes, isto é, iopamidol e iopromida. Finalmente, a afinidade pelo carvão ativado está correlacionada com a estrutura da molécula: estruturas cíclicas, como as de benzotriazol e melamina, adsorvem mais em carvão ativado do que micropoluentes com estruturas lineares ou globulares, como metformina e hexametilenotetramina.

O Capítulo 3 descreve o potencial da biomassa, proveniente de filtros de carvão ativado em escala industrial, para promover a degradação de micropoluentes. Avaliar a biodegradação de micropoluentes na presença de carvão ativado requer uma distinção clara entre adsorção e biodegradação. Neste capítulo, tal distinção é realizada pela remoção de micropoluentes a $5^{\circ} \mathrm{C}$ e $20^{\circ} \mathrm{C}$ com carvão ativado biologicamente ativo ou autoclavado. A $5^{\circ} \mathrm{C}$, os micropoluentes são removidos principalmente por adsorção, enquanto que a $20^{\circ} \mathrm{C}$ eles também podem ser biodegradados. O carvão ativado autoclavado é utilizado para determinar o efeito da temperatura na adsorção. Neste capítulo, foi empregada a mesma mistura de micropoluentes descrita no Capítulo 2. Dentre esses micropoluentes, três são biodegradados pelo biofilme do carvão ativado: iopromida, iopamidol e metformina. Além 
disso, a adsorção de alguns micropoluentes, como iopromida e iopamidol, aumenta com a temperatura, enquanto que a adsorção de outros micropoluentes, como a metformina e a guanilureia, reduz com o aumento da temperatura. Finalmente, é comparada a capacidade de adsorção de carvão ativado previamente utilizado em estações de tratamento de água e virgem. O carvão ativado previamente utilizado apresenta adsorção reduzida para a maioria dos micropoluentes, com exceção daqueles cuja carga é positiva (metformina e guanilureia) e de hexametilenotetramina.

Nos Capítulos 4 e 5 é avaliado o potencial de se utilizar a biodegradação para regenerar a capacidade de adsorção do carvão ativado. Isto foi feito por meio de experimentos em batelada e contínuos, utilizando como modelo o micropoluente melamina e biomassa capaz de degradá-la. A melamina é detectada com frequência em águas superficiais devido à sua ampla utilização e insuficiente remoção em estações convencionais de tratamento de efluentes.

No Capítulo 4, são apresentadas as condições favoráveis à biodegradação de melamina, avaliada em condições óxicas ou anóxicas, aplicadas contínua ou alternadamente. Além disso, o efeito de uma fonte de carbono adicional na biodegradação é determinado. As condições mais favoráveis para a biodegradação de melamina são aplicadas para biorregenerar carvão ativado carregado com melamina. Nos testes em batelada, observou-se que a melamina pode ser biodegradada em condições óxicas e anóxicas, e que a biomassa capaz de degradar melamina pode recuperar pelo menos $28 \%$ da capacidade de adsorção original do carvão ativado. Além disso, altas taxas de adsorção obtidas com o carvão ativado biorregenerado indicam que a biorregeneração ocorre principalmente na fração dos poros maiores do carvão ativado.

No Capítulo 5 é avaliada a contribuição da biodegradação para a remoção total da melamina em filtros de carvão ativado em escala de laboratório. Os filtros são inoculados com a mesma biomassa descrita no Capítulo 4, capaz de degradar melamina. Empregando-se dois métodos de inoculação, também foi avaliado o efeito de uma fonte adicional de carbono (metanol) e do tempo de contato na eficiência na remoção de melamina. A inoculação de filtros de carvão ativado com biomassa capaz de degradar melamina aumenta a eficiência de remoção deste micropoluente em pelo menos $29 \%$ na ausência de uma fonte de carbono adicional. Quando o metanol é utilizado como fonte de carbono adicional nos filtros inoculados, a remoção de melamina é quase completa (até 99\%) e o ponto de ruptura não é 
observado. Ademais, o suprimento de metanol a um filtro inoculado e próximo da saturação pode estimular até $98 \%$ de biorregeneração.

As conclusões e implicações relacionadas aos capítulos experimentais são apresentadas e discutidas no Capítulo 6 e postas em perspectiva ao conhecimento atual sobre remoção de micropoluentes com carvão ativado. Finalmente, são apresentadas recomendações para trabalhos futuros e oportunidades para biorregeneração como forma de melhorar o processo de tratamento de águas. 


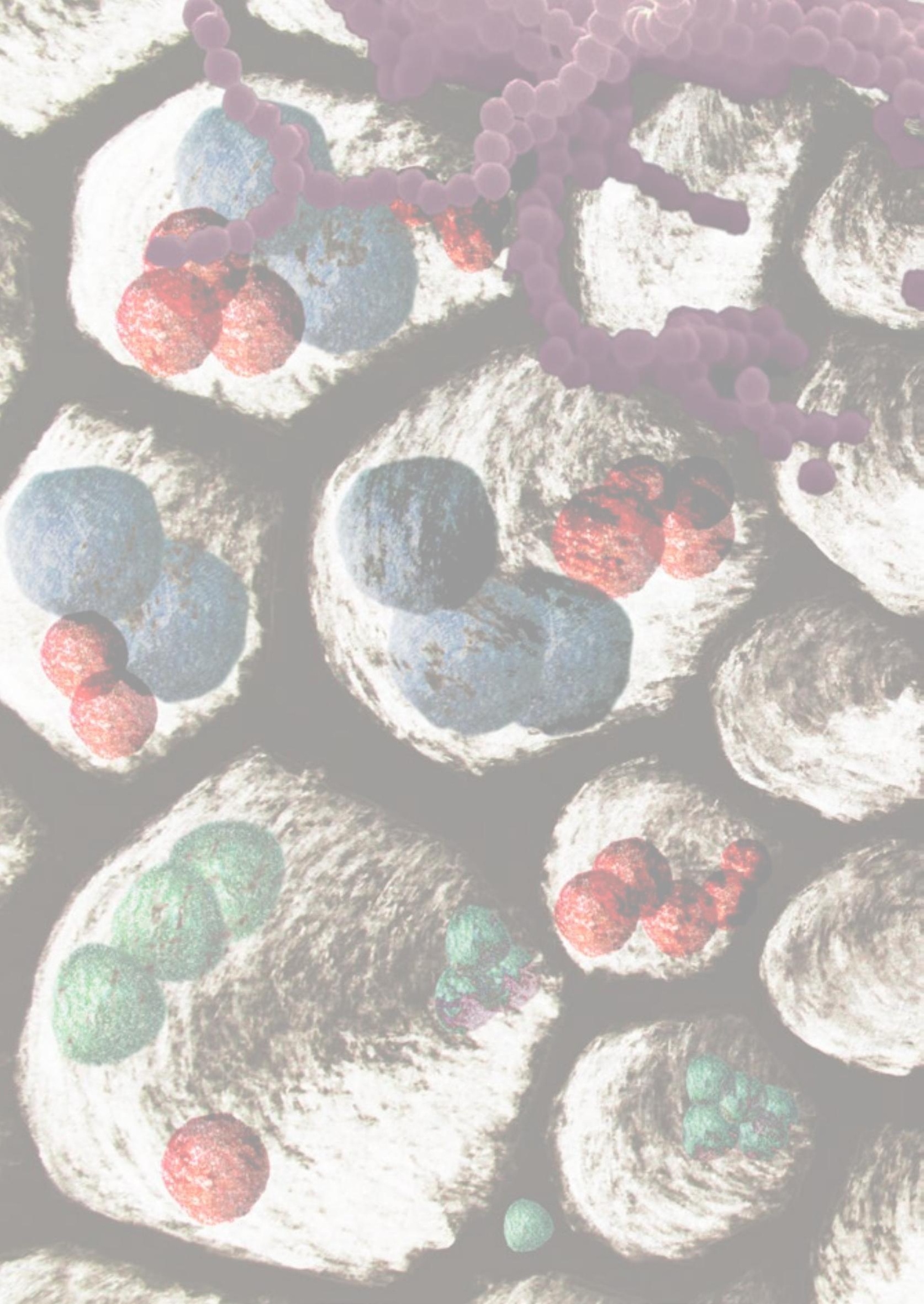


The past years have been an intense journey, which was only made possible (and more cheerful) because of the help and support of countless people. The professional and personal support I received from all of you was essential for the success of this project. Alette and Bert, thank you for guiding me during this journey. Alette, you have the gift of balancing assertiveness and kindness in a way that I always felt supported, encouraged and with the right level of pressure. It has made a great difference to know that I could count on your guidance for all the different aspects of my $\mathrm{PhD}$ and my own development. Thank you for believing in me from the very beginning and making sure I would hear that again whenever I needed. Bert, thank you this opportunity, for all you taught me, for your constructive criticism that would always push me to get the best out of myself.

To all those amazing people I met at ETE, thank you for being part of this journey. Grietje, if it wasn't for that walk around São Paulo, when you asked me if I still considered doing a $\mathrm{PhD}$, I don't think I would be here. Thank you so much for reminding me of that plan and encouraging me to pursue it.

To my dearest office mates, thank you for sharing space and moments. Rosanne, thank you for teaching me how to knit (which proved to be a wonderful coping strategy for stressful periods), for sharing all these great recipes and for eventually accepting me in the middle of the already very busy office. Leire, thank you for making working in the lab at ETE so much fun! I miss your messy bun and the sunflower seed peels all over the floor of what is now office Z.1015. Emilius, thank you for the squash matches, the fun discussions about languages and history and for being such an impressive example of how to finish a thesis in time. Azie, thank you for always being so kind and sweet, and for the calming talks when I needed the most. Dandan, as the senior PhD in the office, you were the example I could look up to, realistically showing me that achieving this was tough, but possible. Margo, bedankt voor de taaluitwisseling bijeenkomsten, die ook als een soort van therapie werken. Annemerel, Yu Lei, Sha Gao, Vincent and Xiaofang Yan, circumstances limited the time we spent together in the office, but nevertheless, I really appreciate the company.

Being part of a great team is essential to produce quality research and have fun while doing it. Thank you to the micropollutants team (Wembo, Yujie, Yu Lei, Elackiya, Jinsong, Koen, Arnoud, Thomas, Andrea, Andrea Brunsch, Azie) for the discussions, ideas, lab clean-ups and fun dinners. Thank you Alette and Nora for creating and maintaining a united, collaborative and friendly team. 
Andrea and Thomas, thank you for being great paranymphs and friends. Andrea, gracias for eventually accepting me as a Latin, for holding my hand when we thought we would die somewhere underwater in Japan, for all the listening and hugging that were often needed. Thomas, thank you for always being available on Skype and Whatsapp to answer my most random questions (even on Saturday mornings). Meetings are certainly more fun when they end with a joke, thank you for remining me of that! Adrian, thank you for all the talks, the advices, for listening to my unfiltered complaints, for accepting me in my grumpiest version and still sticking around. Silvi, you were such an example of how to stay strong and determined when life gets tough! Thank you for sharing this energy with me. Justine, thank you for reading the introduction, discussion, and for being a great friend before and after ETE. Who would have guessed that you would still be my buddy after all this time!

Jouke, thank you for your contribution to the first paper, which was a great push to get me started on this scientific publishing world, and for the collaboration throughout the rest of the PhD. I appreciate the confusing modelling discussions and the fun random talks in between the serious ones. Joeri, Nora, Pim and Adi, thank you for the climbing evenings that certainly helped me relieve all possible stress from work and recharge the batteries for what was left of the week (or sometimes just discharge them entirely...). Nora, thank you for lending me Shabke every now and then, and making working in pandemic times more gezellig.

To the dearest former and current members of the lab team (Livio, Katja, Pieter, Julian, Zoe, Jean, Ilse, Hans, Vinnie, Bert, Femke): despite all the challenges of lab work, you made sure I could still smile and have fun while spending time downstairs. Livio, you are such a pleasant person to work with. Thank you for being so available and realistic when it comes to developing methods and fixing broken LCs, and for always trying to make our work environment (and beyond) more pleasant. I admire this greatly. Jean, I appreciate your support not only at the lab, but also at the coffee corner, making sure I would stay on track with my Dutch learning goals. Katja, thank you for being always available, critical and creative. I admire how you always come up with out of the box ideas. Vinnie, thank you for making the impossible possible and being so approachable. Bert, same goes to you, I really admire this ability you two have to comply with this selection of crazy and random demands of all our projects. 
To Liesbeth, Wies, Gea, Marjolein and all those who integrated the secretary team at some point in the last 4.5 years, thank you all for your support, for making sure this whole process could go as smooth as possible.

To all other friends and colleagues from ETE (Dainis, Tania, Carlos, Sannita, Kasper, Momo, Pradip, Ilse, Bignan, Farzaneh, Delaram, Shokouh, Fatma, Selin, Dilan, Jess, Shiyang, Kamonasish, Indra, Merijn, Baptiste, Yvonne, Lucia, Ivonne, Sanne, Darja), the talks and shared moments during coffee breaks, borrels and every other occasion were essential to give me energy and ideas to continue moving forward. Momo, I won't forget the look on your face when you turned around and saw me in the coffee corner... no, you were not going crazy, I was indeed back.

To all MSc thesis students I had the opportunity to supervise, Esther, Mahesa, Krishanu, Anna, Vanessa, Wesley, Toshi, Chufeng, Mingyi and Yuchen. Not only was your work crucial to complete this thesis, but I learned a lot from working together with you. Thank you for giving me the chance to guide you during your theses and for making my work more pleasant. Esther, your energy and enthusiasm was a great kick start to this project! Wesley, you came into this project exactly at the right time to help me to make those columns work, I appreciate this greatly! To all of you, thank you for joining me in part of this trajectory.

To those outside ETE who were of great importance to this work: Marco Blokland, thank you for developing the method that could analyze the "mixture from hell" and for helping me to overcome my fear of exploding a half million-euro machine. David de Ridder, thank you for always helping me with all the information I needed plus a little extra and for your encouraging words on my first manuscript. Nadine Boelee, thank you for joining this journey with all your experience and enthusiasm. Your kindness and critical thinking made our meetings pleasant and productive. Roberta Hofman, Wolter Siegers, Cheryl Bertelkamp, Nikki van Bel and others from KWR who I had the opportunity to meet and discuss this work with, thank you for sharing your experience and ideas with me.

Nat, what can I say? You were so patient to review all my motivation letters and kept me eager for coming back, you made sure I remained hopeful even after so many failed attempts. Thank you for being such a sweet and loyal friend since the very beginning. You are a great example of how friends can become your family, especially when we're away from home. Thank you for making my process of coming back to Wageningen smoother, for 
sharing your home with me, for bringing me fresh fruit for the train trip back from the airport and putting flowers on the bedside table and, last but not least, for teaching me $\mathrm{R}$ on the free time. Dina, I cannot thank you enough for designing the cover of this thesis and for that amazing and extensive email with so many practical tips on how to prepare the book. If it wasn't for that, I'd probably still be sitting and waiting for a giant pdf to be born. Natuurlijk, I also can't forget to thank you for turning our bathroom into a disco, for tricking us with fake post cards and not missing a single opportunity for making me laugh.

Marta and Lucille, I miss so much having such sweet neighbors! Thank you for the talks in the corridor, for helping us to finish random drinks left in the apartment for generations, for inspiring me to go on bike trips and teaching me how to change a flat tire! Jaap, Josi, Sophie, Alice and Fiona, thank you for opening your home to us, for all the amazing dinners and the fun talks.

À minha família, obrigada por todo o apoio e força sem os quais nada disso seria possível. Mama e papi, obrigada por me darem asas, por me encorajarem a seguir meus sonhos, mesmo que isso significasse que ficaríamos tão longe um do outro. $\mathrm{O}$ apoio de vocês à essa decisão e o amor incondicional que recebo sempre foram essenciais para me manter motivada e forte. Obrigada por terem me ensinado a ser crítica, questionadora e um pouquinho nerd, na dose certa (ou não, depende do referencial). Kiko, quem sabe o que teria sido de nossos destinos se não fosse aquela viagem em 2008 pra aprender inglês? Além de nos divertirmos horrores, essa decisão nos abriu tantas portas! Obrigada por ser um grande parceiro para as aventuras da vida. Vi, minha irmã e segunda orientadora, minha professora de holandês e terapeuta, minha modelo de mulher maravilha. Obrigada por me ouvir, por me ensinar tanto sobre tanta coisa, por me ajudar a acreditar em mim mesma. Sem as nossas conversas e os seus conselhos esse livro não existiria. Kris, thank you for never answering any question with a simple answer, for keeping me sharp in every discussion, and, of course, for helping me with my very first R scripts! Neide e Gilberto, meus sogros, muito obrigada pelo apoio incondicional, desde o começo. Obrigada por me convencer, Neide, a adiar a entrevista alguns dias pra eu poder faze-la com calma e mostrar o meu melhor. Eu sei que hoje a filha de vocês mora longe e sou parcialmente responsável pela saudades que todos sentem. Apesar disso, vocês demonstram constantemente o apoio por essa nossa decisão, o que torna a saudade mais tolerável.

Débora, meu amor, o que dizer sobre essa parceria? Que eu queria colocar seu nome como co-autora em algum lugar nessa tese. Obrigada pela paciência para me ouvir praticar tantas 
apresentações repetidas vezes, por revisar minha introdução e discussão e inúmeras outras partes dessa tese na reta final. Com sua empolgação ao achar um errinho aqui e outro ali era impossível eu ficar brava ao gerar o centésimo pdf de um documento. Obrigada por me escutar e me dar colo todos os dias em que eu precisei, por me manter motivada e me fazer acreditar em mim mesma. O seu filtro rosa foi mais do que necessário pra fazer tudo acontecer.

Job dos Reis, a sua ajuda nas últimas fases desse projeto foi essencial para garantir a minha saúde emocional e enfrentar todos os desafios que a vida me apresentou. Obrigada por ser um excelente profissional e por ter me ensinado tanto sobre mim mesma. Oda e Piru, obrigada por terem lido e corrigido o resumo dessa tese em português e garantir que eu não passasse vergonha ao escrever na minha língua materna. A todos os outros amigos brasileiros aqui na Holanda, em outros cantos da Europa e no Brasil, obrigada por fazerem parte da minha família extendida. Sua amizade e carinho foi e continua sendo essencial para fazer com que esses anos longe de casa sejam mais felizes. 


\section{About the author}

Laura Magalhães Piai was born and raised in the big city of São Paulo, Brazil on December $5^{\text {th }}, 1985$. Laura obtained a Bachelor of Science degree in biology from the University of Sao Paulo, completing her thesis at the Environmental Microbiology Laboratory, where she studied methanotrophic bacteria from river sediments. It was there that Laura started to become passionate for science. Before completing her degree, Laura took a gap year and travelled to Australia for a year to experience living abroad for the

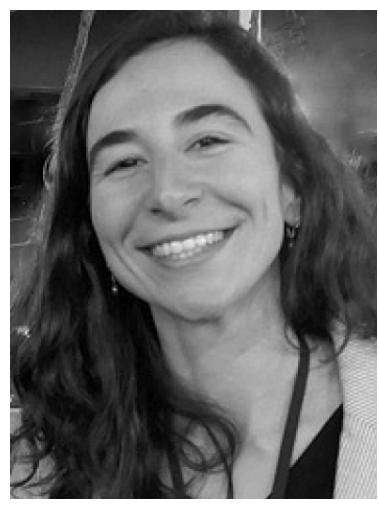
first time and improve her English proficiency. In 2010, Laura completed her BSc degree and moved to the Netherlands to begin her Masters of Science in Molecular Life Sciences at Wageningen University. During this program, Laura developed a strong affinity for studying water and wastewater treatment technologies. During her MSc thesis, she worked with anaerobic methane oxidation coupled to nitrite reduction at the Environmental Technology group. For her internship, she decided to take up a challenge, and moved for 4 months to Tanzania to monitor the performance of an anaerobic digester treating human excreta and producing biogas for a school kitchen. After completing her MSc studies in 2012, Laura moved back to Brazil and, soon after, started working as an Environmental Specialist for Akzo Nobel. However, Laura's passion for science encouraged her to change the direction of her career and pursue a doctorate degree in the Netherlands. In 2016, she moved back to Wageningen and started her PhD journey, studying the removal of micropollutants from drinking water using activated carbon at the Environmental Technology group. Currently, Laura is a postdoctoral researcher at the same group, continuing her studies on micropollutants removal from water and wastewater. In her free time, Laura enjoys going on long walks and bike trips, cooking and knitting. 


\section{Publications}

Piai, L., Blokland, M., van der Wal, A., Langenhoff, A., 2020. Biodegradation and adsorption of micropollutants by biological activated carbon from a drinking water production plant. Journal of Hazardous Materials 388, 122028.

Piai, L., Dykstra, J.E., Adishakti, M.G., Blokland, M., Langenhoff, A.A.M., van der Wal, A., 2019. Diffusion of hydrophilic organic micropollutants in granular activated carbon with different pore sizes. Water Research 162, 518-527.

Kampman, C., Piai, L., Temmink, H., Hendrickx, T.L.G., Zeeman, G., Buisman C.J.N., 2018. Effect of low concentrations of dissolved oxygen on the activity of denitrifying methanotrophic bacteria. Water Science \& Technology 26(11), 2589-2597.

Kampman, C., Piai, L., Hendrickx, T.L.G., Temmink, H., Zeeman, G., Buisman, C.J.N., 2014. Effect of temperature on denitrifying methanotrophic activity of 'Candidatus Methylomirabilis oxyfera'. Water Science \& Technology 70(10): 1683-1689.

Publications in review and in preparation

Piai, L., van der Wal, A., Boelee, N., Langenhoff, A. Melamine degradation to bioregenerate granular activated carbon. (in review)

Piai, L., Langenhoff, A., de Wilde, V., van der Wal, A. Biodegradation of melamine in activated carbon filters resulting in bioregeneration. (in preparation) 


\section{SENSE}

Netherlands Research School for the

Socio-Economic and Natural Sciences of the Environment

\section{I P L O M A \\ for specialised PhD training}

The Netherlands research school for the

Socio-Economic and Natural Sciences of the Environment

(SENSE) declares that

\section{Laura Piai}

born on 5 December 1985 in São Paulo, Brazil

has successfully fulfilled all requirements of the educational PhD programme of SENSE.

Wageningen, 5 February 2021
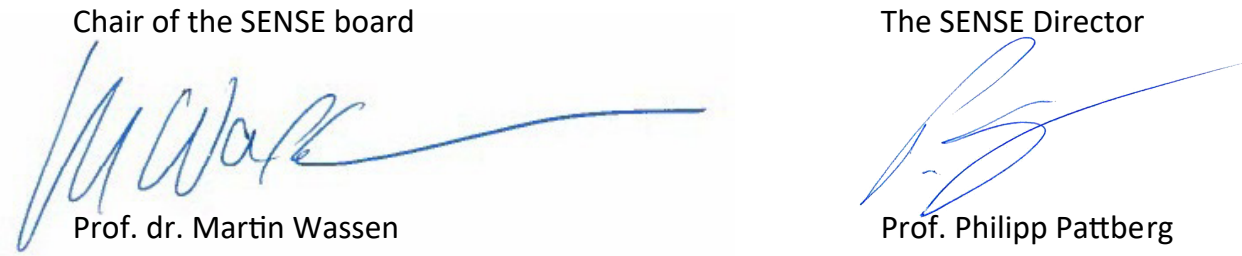

The SENSE Research School has been accredited by the Royal Netherlands Academy of Arts and Sciences (KNAW)

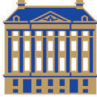

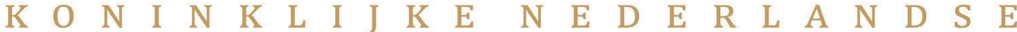

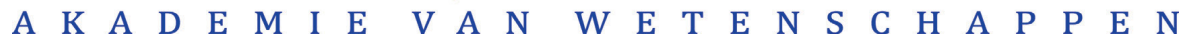




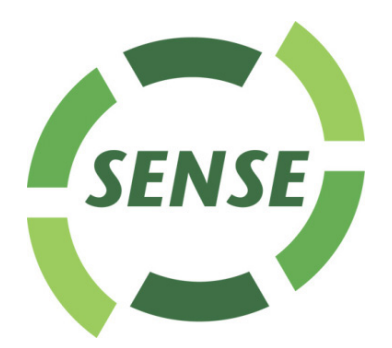

The SENSE Research School declares that Laura Piai has successfully fulfilled all requirements of the educational PhD programme of SENSE with a work load of $33.5 \mathrm{EC}$, including the following activities:

\section{SENSE PhD Courses}

- Environmental research in context (2016)

- Stable isotope applications in microbiology and environmental studies (2017)

o Research in context activity: 'Initiating, organizing and coordinating successful study trip to Chile for PhD candidates from the Dept. of Environmental Technology (2-16 March 2019)'

\section{Other PhD and Advanced MSc Courses}

- Basic statistics, PE\&RC and WIMEK graduate schools (2016)

- Introduction to R for statistical analysis, PE\&RC and WIMEK graduate schools (2017)

- Linear models, PE\&RC and WIMEK graduate schools (2017)

- Project and time management, Wageningen Graduate Schools (2017)

- Scientific publishing, Wageningen Graduate Schools (2017)

- Scientific writing, Wageningen Graduate Schools (2018)

- Teaching and supervising thesis students, Wageningen Graduate Schools (2018)

- Competence assessment, Wageningen Graduate Schools (2019)

- Brain training, Wageningen Graduate Schools (2019)

\section{Management and Didactic Skills Training}

o Supervising 10 MSc students with thesis (2016-2020)

- Supervising 1 BSc student with thesis (2019)

\section{Oral Presentations}

- Can biodegradation of micropollutants lead to bioregeneration of activated carbon? Water Science for Impact, 16-18 October 2018, Wageningen, The Netherlands

- Diffusion of micropollutants in granular activated carbon with different pore sizes, 11th Micropol \& Ecohazard Conference, 21-24 October 2019, Seoul, South Korea

- Technologies for removing micropollutants from water and wastewater, Technological Approaches for Future (Waste)Water Treatment and Resource Recovery, 6 March 2019, Santiago, Chile

- Melamine adsorption and biodegradation in granular activated carbon filters, Environmental Technology for Impact 2020, 3-4 June 2020, Online

SENSE coordinator PhD education

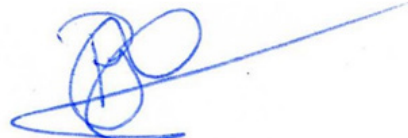

Dr. ir. Peter Vermeulen 
The research described in this thesis was financially supported by Evides Water Company N.V. (Rotterdam, The Netherlands).

Financial support from Wageningen University for printing this thesis is gratefully acknowledged.

Cover design by Dina in ' $t$ Zandt

Printed by Proefschriftmaken on FSC-certified paper 
